\title{
Les organes restreints des organisations internationales
}

Citation for published version (APA):

Ozigré, P. M. (2013). Les organes restreints des organisations internationales. [, Maastricht University]. Uitgeverij BOXPress. https://doi.org/10.26481/dis.20130627po

Document status and date:

Published: 01/01/2013

DOI:

10.26481/dis.20130627po

Document Version:

Publisher's PDF, also known as Version of record

\section{Please check the document version of this publication:}

- A submitted manuscript is the version of the article upon submission and before peer-review. There can be important differences between the submitted version and the official published version of record.

People interested in the research are advised to contact the author for the final version of the publication, or visit the DOI to the publisher's website.

- The final author version and the galley proof are versions of the publication after peer review.

- The final published version features the final layout of the paper including the volume, issue and page numbers.

Link to publication

\footnotetext{
General rights rights.

- You may freely distribute the URL identifying the publication in the public portal. please follow below link for the End User Agreement:

www.umlib.nl/taverne-license

Take down policy

If you believe that this document breaches copyright please contact us at:

repository@maastrichtuniversity.nl

providing details and we will investigate your claim.
}

Copyright and moral rights for the publications made accessible in the public portal are retained by the authors and/or other copyright owners and it is a condition of accessing publications that users recognise and abide by the legal requirements associated with these

- Users may download and print one copy of any publication from the public portal for the purpose of private study or research.

- You may not further distribute the material or use it for any profit-making activity or commercial gain

If the publication is distributed under the terms of Article $25 \mathrm{fa}$ of the Dutch Copyright Act, indicated by the "Taverne" license above, 


\section{Les Organes Restreints \\ des Organisations Internationales}

P.M. OZIGRÉ 
C P.M. OZIGRÉ, Maastricht 2013

Printed \& lay-out by: Proefschriftmaken.nl || Printyourthesis.com Published by: Uitgeverij BOXPress, Oisterwijk 


\title{
Les Organes Restreints des Organisations Internationales
}

\author{
DISSERTATION \\ To obtain the degree of Doctor at Maastricht University, \\ on the authority of the Rector Magnificus, Prof. dr. L.L.G. Soete
}

in accordance with the decision of the Board of Deans, to be defended in public

on Thursday 27 June 2013, at 12.00 hours

by

P.M. OZIGRÉ 


\section{Supervisors}

Prof. Dr. Michael G. Faure LL.M.

Prof. Francisco Djedjro Melèdje (l’Université Félix Houphouet Boigny d’Abidjan-Cocody, Abidjan, Republic of Côte d'Ivoire)

\section{Assessment Committee}

Prof. dr. C.A. Schwarz (chairman)

Prof. mr. F.A.M. Stroink

Prof. A.P. van der Mei 


\section{REMERCIEMENTS}

"Toute vie humaine commence à se construire par l'imitation. On vit en croyant et en imitant les autres. Ceci est vrai dans l'arrangement floral, le judo, l'escrime...

Les gens peuvent avancer en suivant les instructions de leur maître, et tout en l'imitant, on commence à sélever au stade supérieur de la créativité. C'est ainsi que l'on trouve sa propre façon de vivre. »

TSUNESABURO MAKIGUCHI

Cette thèse est loccasion inouie d'exprimer mon infinie gratitude à mes maitres.

Je pense en premier lieu au Professeur Melèdje DJEDJRO de l'Université Felix HOUPHOUET-BOIGNY de Cocody Abidjan. J'ai suivi ses enseignements en droit constitutionnel (première année), sociologie politique (licence), relations diplomatiques (maîtrise), droit international humanitaire (DEA).

Pour cette thèse, j’ai bénéficié de son encadrement et de ses conseilles très avisés.

C’est aussi grace à lui que j’ai pu obtenir la co-direction du Professeur Michael FAURE de l'Université de Maastricht aux Pays Bas.

Je tiens à spécialement remercier le Professeur Michael FAURE, pour ses critiques objectives et constructives durant la préparation de ce travail. Il a fait preuve d'une grande générosité scientifique à mon égard.

Je pense aussi à ma famille, surtout à feu mon père OZIGRE Koffi Alphonse et à mes deux filles Alexia et Kendra qui ont été une source de motivation dans la rédaction de ma thèse.

Je dédie ce travail à tous mes amis et particulièrement à mes compagnons membres de la Soka Gakkaï Internationale (Association des Bouddhistes Laïques). 
SOMMAIRE

\section{INTRODUCTION}

PREMIERE PARTIE: Les fondements de l'institution des organes restreints dans les organisations internationales

Titre I : La recherche de l'efficacité des organisations internationales 39

Chapitre I : Les apports fonctionnels des organes restreints dans les organisations internationales.

Chapitre II : Les apports au plan politique des organes restreints au fonctionnement des organisations internationales

Titre II : $\quad$ La problématique de l'hégémonie des États membres dans les organes

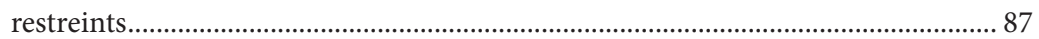

Chapitre I : Lémergence des oligarchies d'États dans les organes restreints ................................. 88

Chapitre II : La contestation de l'hégémonie des grandes puissances dans les organes restreints

DEUXIEME PARTIE: L’ordonnancement des pouvoirs des organes restreints 147

Titre I : La détermination des pouvoirs des organes restreints............................................. 149

Chapitre I : Les pouvoirs politiques des organes restreints....................................................... 150

Chapitre II : Les pouvoirs juridiques des organes restreints. ........................................................ 177

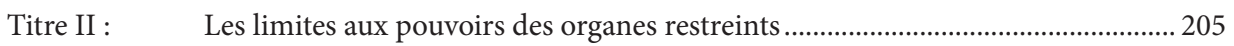

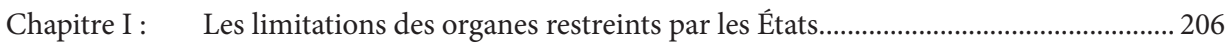

Chapitre II : Les limitations des pouvoirs des organes restreints par les organes pléniers........ 231 


\section{ABREVIATIONS DES ORGANISATIONS INTERNATIONALES.}

B.I.T : Bureau International du Travail.

CEDEAO : Communauté économique des États de l'Afrique de l'Ouest.

C.I.J : Cour Internationale de Justice.

C.J.C.E $\quad$ : Cour de Justice des Communautés Européennes.

C.P.J.I : Cour Permanente de Justice Internationale.

F.M.I : Fonds Monétaire International.

L.E.A : : Ligue des États Arabes.

O.A.C.I : Organisation de l'Aviation Civile Internationale.

O.E.A : Organisation des États Américains.

O.I.T : Organisation Internationale du Travail.

O.N.U : Organisation des Nations Unies.

O.U.A : Organisation de l'Unité Africaine.

S.D.N : Société Des Nations.

T.A.N.U : : Tribunal Administratif des Nations Unies.

T.A.O.I.T : Tribunal Administratif de l'Organisation internationale du Tra vail

U.A : Union Africaine.

U.E : Union Européenne.

U.N.E.S.C.O : Organisation des Nations Unies pour l'Education, la Science et la Culture. 


\section{ABREVIATIONS UTILISEES DANS LA BIBLIOGRAPHIE}

$\begin{array}{ll}\text { A.F.D.I } & \text { : Annuaire Français de Droit international. } \\ \text { L.G.D.J.I } & \text { : Librairie Générale de Droit et de Jurisprudence. } \\ \text { P.U.C.I } & \text { : Presses Universitaires de Côte d'Ivoire. } \\ \text { P.U.F } & \text { : Presses Universitaires de France. } \\ \text { R.B.S.J } & \text { : Revue Béninoise des Sciences juridiques et Administratives } \\ \text { R.C.A.D.I } & \text { : Recueil des Cours de l’Académie de Droit International. } \\ \text { R.I.D } & \text { : Revue Ivoirienne de Droit. } \\ \text { R.G.D.I.P } & \text { : Revue Générale de Droit International Public. } \\ \text { S.F.D.I } & \text { : Société Française pour le Droit International. }\end{array}$




\section{INTRODUCTION}

Le sujet sur lequel porte cette étude s'intitule : «Les organes restreints des organisations internationales». Pour une meilleure approche de cette question, ces propos introductifs sarticuleront en trois points essentiels.

Il s’agira au préalable de déterminer l'objet de l'étude (I), puis de relever les éléments de sa problématisation (II). Enfin, la précision de l'intérêt du sujet et de l'approche méthodologique s'avère indispensable pour une meilleure compréhension du sujet (III).

\section{I/ LA DETERMINATION DE L'OBJET DE L'ETUDE}

Le Professeur Madeleine GRAWITZ définit l'objet comme « le sujet de la connaissance scientifique ». ${ }^{1}$ Déterminer l'objet d'une étude scientifique revient à dire ce sur quoi elle porte. De ce fait, il convient de donner un contenu juridique à la notion d’organisation internationale (A) et à celle d’organes restreints (B).

\section{A : LA NOTION D'ORGANISATIONS INTERNATIONALES}

Il est nécessaire de partir de généralités sur les organisations internationales (A), puis d’opérer leurs classifications (B).

\section{1) GENERALITES SUR LES ORGANISATIONS INTERNATIONALES}

Si l'existence des États apparaît comme une constante remarquable dans l'histoire des relations internationales, la naissance et le développement des institutions internationales constituent un des traits remarquables de la société internationale contemporaine. ${ }^{2}$ De façon très schématique, il est possible de distinguer, historiquement, trois générations d’organisations internationales. ${ }^{3}$

La première génération apparaît au début du $19^{\mathrm{e}}$ siècle : il s'agit d’organisations internationales à caractère technique qui apparaissent dans des domaines bien déterminés n'ayant pas au premier chef de coloration politique. ${ }^{4}$ On peut citer les Commissions fluviales telles que la Commission Centrale du Rhin, créée par la Convention de Mayence de 1831 et la Commission Européenne du Danube instituée par le traité de Paris (France) de 1856.

GRAWITZ (Madeleine), Méthodes des sciences sociales, Paris, Dalloz, 1996, p.3.

MERLE (Marcel), La vie internationale, Collection U, $3^{\mathrm{e}}$ édition, Paris, Armand Collin, 1970, p. 57.

GONIDEC (Pierre- François), Institutions internationales, Les cours de droit, Fascicule I, mai, 1971, p.97.

Ibid. 
La deuxième génération est contemporaine à la première guerre mondiale. Pour la première fois, on voit apparaître sur la scène internationale une organisation internationale à caractère politique : la Société des Nations créée le 28 juin 1919. Ce qui implique qu’lle a compétence pour intervenir dans toute espèce de domaines. ${ }^{5}$

La troisième génération apparaît au lendemain de la deuxième guerre mondiale. Ce qui caractérise cette période, c'est la multiplication dorganisations internationales de tous genres et de toutes dimensions. À titre d'exemples nous pouvons citer l'Organisation des Nations Unies (ONU) créée le 25 juin 1945 à San Francisco (États-Unis), l’Agence Internationale de l'Energie atomique (AIEA) dont le Statut a été approuvé le 26 octobre 1956 lors d'une conférence tenue au siège de l'ONU à New York (États-Unis), l'Organisation du traité de l'atlantique nord (OTAN) créée par le traité du 4 avril 1949 liant douze États occidentaux en vue de sauvegarder la paix et la sécurité.

Le Professeur René-Jean DUPUY considère qu' " avec l'avènement des organisations internationales, nous passons du monde des cités à la cité du monde. Nous évoluons d'un stade purement interétatique reposant sur une coordination occasionnelle des politiques gouvernementales, à une société organisée. On tend à accéder à un système institutionnel s'inspirant d'une conception différente, susceptible de retrouver, dans une certaine mesure les caractères du droit interne et d'offrir l'image d'un ensemble doté d’organes compétents pour légiférer, juger et exécuter.» ${ }^{6}$

Cette mutation de la structure de la société internationale s'est opérée sous l'influence de certains facteurs. D’une part, le nombre d'États s'est multiplié en un siècle, en même temps que s'intensifiaient leurs différences de pouvoir, de développement économique et de valeur culturelle.? D’autre part, de nouveaux sujets de droit ont commencé à faire leur apparition aux cotés de l'État, sujet de droit classique, pour répondre aux exigences de la vie internationale. Parmi ces nouveaux sujets figurent les organisations internationales. ${ }^{8}$ Elles se sont progressivement imposées au cours du XXe siècle où leur champ d'action s'est étendu et diversifié, jusquà couvrir presque toutes les activités humaines. Aussi, leur nombre dépasse-t-il largement le chiffre de trois cents et elles sont devenues, le symbole d'une communauté politique institutionnalisée. ${ }^{9}$

Les organisations internationales constituent des sujets à part entière du droit international public, au même titre que les États. De ce fait, elles ne sont pas en marge de la Ibid. Ibid. DIEZ DE VELASCO VALLEJO (Manuel), Les organisations internationales, Paris, Economica, 1999, p.3. 
communauté internationale. ${ }^{10}$ Bien au contraire, elles sont venues y rejoindre automatiquement, en tant que membres de cette communauté et sujets de son droit, les membres primaires représentés par les appareils souverains des collectivités étatiques. L'extrême diversité des organisations internationales, conduit à ce qu'une définition générale, caractérisant l'institution en retenant ses éléments communs, ne peut être que de caractère intellectuel ou doctrinal. ${ }^{11}$ Aux fins de la présente étude, on définira les organisations internationales comme " des associations d'États créées par un traité constitutif et dotées d'une personnalité juridique distincte de celle des États membres, disposant dorganes propres aux fins de réaliser des objectifs communs.» ${ }^{12}$

La notion dorganisation internationale est une expression désignant parfois la situation dans laquelle les nations ont entre elles des rapports juridiquement ordonnés $;^{13}$ une collectivité composée d'États ou d’autres collectivités qui ne relèvent pas du même État, poursuivant un intérêt commun au moyen dorganes qui lui sont propres. ${ }^{14}$ De cette approche définitionnelle du concept d’organisation internationale, des précisions s'imposent. Ainsi, la notion de traité constitutif mérite une analyse approfondie. La Convention de Vienne sur le droit des traités du 23 mai 1969 dispose en son article $\mathbf{2}$ paragraphe $\mathbf{1}$ alinéa (a) que «l'expression " traité » s'entend d'un accord international conclut par écrit entre États et régi par le droit international, qu’il soit consigné dans un instrument unique ou dans deux ou plusieurs instruments connexes, et quelle que soit sa dénomination particulière; $»^{15}$

Pour les Professeurs NGUYEN QUOC Dinh, Patrick DAILLIER, Alain PELLET et Mathias FORTEAU, le traité dans la théorie du droit international public, désigne « tout accord

10 La notion de communauté internationale, selon le Juge Roberto AGO « désigne en générale une collectivité humaine qui se distingue avant tout par le fait d'avoir pour membres primaires des entités non pas physiques, mais politiques et souveraines. Ce sont les États qui sont les membres primaires de la communauté internationale. Cependant, avec le développement du volume des relations entre les États, sont apparues les organisations internationales, qui ne sont pas restées en marge de cette communauté. Bien au contraire, elles sont venues y rejoindre les États. Par ailleurs, cette communauté se caractérise par trois aspects. D’abord, c'est une communauté naturelle, c'est-à-dire une collectivité dont nous constatons l'existence dans les faits, qui s'est formée par le développement spontané entre ses membres de liens sociaux, de relations stables et organisées et non pas une collectivité artificielle, dans le sens qu’à l'origine de son existence il y aurait un acte de volonté. Ensuite, il faut noter l'existence d'une seule et unique communauté internationale. Enfin, elle s'est constituée d'entités rigoureusement jalouses de leur égale souveraineté. De ce fait, elle est une collectivité qui refuse toute hiérarchie entre ses membres, elle est dit-on, une société de subordination. De ce même fait, la communauté internationale universelle est et ne peut être qu'une collectivité typiquement non institutionnalisée.» (Voir Roberto AGO, "Communauté internationale et organisation internationale ", in DUPUY (René-Jean) (dir.), Manuel sur les organisations internationales, Martinus Nijhoff publishers, Dordrecht/ Boston/ Lancaster, 1988.)

11 COMBACAU (Jean) et Sur (Serge), Droit international public, $9^{\mathrm{e}}$ édition, Paris, Montchrestien, 2010, p.708.

12 NGUYEN QUOC (Dinh), DAILLIER (Patrick), FORTEAU (Mathias), PELLET (Alain), Droit international public, $8^{\mathrm{e}}$ édition, LGDJ, Paris, 2009, p.572.

13 REUTER (Paul), "Quelques réflexions sur le vocabulaire du droit international », in Mélanges offerts au Doyen TROTABAS, p.12.

14 Union Académique Internationale, Dictionnaire de la terminologie du droit international, Paris, Sirey, 1960, p.28.

15 Voir l’article 2 paragraphe 1 alinéa (a) de la Convention de Vienne sur le droit des traités du 23 mai 1969. 
conclu entre deux ou plusieurs sujets du droit international, destiné à produire des effets de droit et régit par le droit international public $»{ }^{16} \mathrm{Si}$ cette définition a le mérite de mettre en évidence le sens général du traité, il faut cependant souligner que le traité constitutif des organisations internationales recèle des particularités.

Dans sa nature juridique, le traité constitutif d'une organisation internationale revêt la forme d'un traité multilatéral négocié au cours d’une Conférence internationale, signé par des plénipotentiaires des États contractants, ratifié et entré en vigueur selon les principes classiques des accords internationaux. ${ }^{17} \mathrm{Du}$ point de vue formel, le traité constitutif se singularise par son multilatéralisme. Au niveau de son régime juridique, il est important de souligner qu'il est la Constitution de l'organisation internationale. Autrement dit, l'acte juridique qui donne naissance et organise les structures et le fonctionnement de la nouvelle entité juridique qui vient dêtre créée. ${ }^{18}$

Les membres originaires de l'ONU ont instauré la primauté de la Charte sur tous les autres traités conclus par les États membres. Aussi, les rédacteurs de la Charte ont-ils expressément introduit, à l'article 103, une disposition spéciale relative aux conflits d’obligations. Selon cette disposition, " en cas de conflit entre les obligations des membres des Nations Unies en vertu de la présente Charte et leurs obligations en vertu de tout autre accord, les premières prévaudront ». Il s'agit d'une règle aussi simple et efficace assurant la primauté de la Charte dans tous les cas de conflits possibles avec un autre accord international. ${ }^{19}$

La Convention de Vienne sur le droit des traités de 1969 s'oriente aussi dans le sens de la primauté de l'acte constitutif. Cette primauté ressort explicitement de son article 5 qui dispose : "La présente Convention s'applique à tout traité qui est l'acte constitutif d'une organisation internationale et à tout traité, adopté au sein d’une organisation internationale, sous réserve de toute règle pertinente de l'organisation.» Cela signifie que les traités constitutifs se rangent dans une catégorie particulière, en ce sens qu'ils peuvent, mais ne doivent pas nécessairement se soumettre aux normes de la Convention de Vienne. ${ }^{20}$

La notion de traité constitutif ainsi définie implique que les organisations internationales sont des émanations des États. Conscients de l’ampleur des tâches à réaliser, les États décident d'unifier leurs efforts en vue d'atteindre des objectifs communs. Cette volonté d'association se traduit par la mise en place dorganisations internationales. De ce fait, les organisations internationales sont des instruments importants pour la coopération entre les États. C’est d'ailleurs ce que souligne le NGUYEN QUOC (Dinh), DAILLIER (Patrick), FORTEAU (Mathias), PELLET (Alain), Droit international public, op.cit.,p.132. MONACO (Riccardo), « Les principes régissant la structure et le fonctionnement des organisations internationales », RCADI, Tome 156, 1977, p.101. Ibid, p. 101 à 108. TOUBLANC (Alix), «Larticle 103 et la valeur juridique de la Charte des Nations Unies », RGDIP, 2004-2, p.439.

20 MONACO (Riccardo), "Réflexions sur la structure et le fonctionnement des organisations internationales », Op.cit., p.103. 
Professeur ABBOTT en ces termes : " while international institutions rarely have strong coercitive powers, institutionalists have demonstrated that relatively modest actions - such as producing unbiased information, reducing the transactions cost of interactions, pooling resources, monitoring states behavior, and helping to mediate disputes - can help states achieve their goals by overcoming structural barriers to cooperation. $»^{21}$ Les organisations internationales permettent aux Etats de réaliser leurs objectifs communs.

Elles existent par la volonté des États. Toutefois, contrairement aux États, les organisations internationales sont dépourvues de la souveraineté. ${ }^{22}$ La souveraineté de l'État est entendue en droit comme un principe selon lequel l'État n'est soumis à aucune autorité supérieure, ni à aucun pouvoir politique concurrent. ${ }^{23}$ Cette notion fait de l'État le sujet principal du droit international public. C’est en cette qualité que les États créent les organisations internationales. Par conséquent, ces derniers ont un statut de sujet dérivé de droit international public. L'organisation internationale apparaît comme un phénomène secondaire par rapport au phénomène étatique; elle est la créature et les États en sont les créateurs. ${ }^{24}$ C’est sans doute la raison pour laquelle le Professeur Francis WODIÉ a pu écrire que "les organisations internationales sont, dans la communauté internationale des sujets, des unités irréductibles aux États. Ceux-ci sont des sujets originaires du droit international et possèdent une personnalité objective. La personnalité des organisations internationales est toute relative dans la mesure où elle naît d'un traité qui est un " res inter alios acta » à l'égard des États tiers ». La personnalité de l'organisation internationale ne se révèle à leur égard quà la suite d'une reconnaissance. Alors que la reconnaissance d'un État est un acte déclaratif, la reconnaissance d'une organisation internationale est un acte constitutif $» .{ }^{25}$ Mieux, les organisations internationales sont régies par le principe de la spécialité. En vertu de ce principe, elles ne peuvent prendre en charge que les activités pour lesquelles elles ont été créées. ${ }^{26}$ Dans une étude relative aux organisations internationales, ${ }^{27}$ le Professeur Charles CHAUMONT exprime le point de vue que toute organisation internationale est spécialisée dans la mesure où elle tient ses compétences des États membres qui n’ont pas renoncé à leur souverainté. C’est sur le fondement de ce principe que s'effectue la répartition des compétences entre les États membres et l'organisation versity of Illinois Law Review, Volume ..., 2008, p.6. organisations internationales, Académie de droit international de La Haye, 1998, p.14.

23 LE JEUNE (Pierrick), Introduction au droit des relations internationales, LGDJ, Paris, 1994, p.18.

24 DUPUY (René-Jean), «État et organisations internationales », op.cit.p.14.

25 WODIE (Francis), Les institutions internationales régionales en Afrique occidentale et centrale, LGDJ, Paris, 1970, p.61.

26 YAO-N'DRE (Paul), Droit des organisations internationales, Abidjan, PUCI, 1996, p.101.

27 CHAUMONT (Charles), «La signification du principe de spécialité des organistions internationales ", in Mélanges ROLLIN, Pédone, Paris, 1964, p.55-66. 
internationale ; entre l'organisation internationale et les autres organisations internationales ; entre les organes d'une organisation internationale. ${ }^{28}$

Les organisations internationales sont donc régies par des principes qui guident leur fonctionnement. Mais au délà des règles de fonctionnement, quelle typologie des organisations internationales pouvons-nous établir?

\section{2) CLASSIFICATION DES ORGANISATIONS INTERNATIONALES}

Les organisations internationales qui déploient leurs activités sur la scène internationale sont variées et diversifiées. La combinaison de différents critères permet de marquer les caractéristiques de chaque organisation particulière et de l'inscrire dans une typologie. ${ }^{29}$ De façon générale, trois critères sont utilisés dans la doctrine pour établir la classification des organisations internationales : le critère de la composition, le critère de l'objet et le critère des pouvoirs. ${ }^{30}$

Selon le critère de la composition, on distingue entre les organisations universelles d'une part, et les organisations régionales, d'autre part.

Les organisations internationales universelles sont celles qui ont vocation à associer tous les États du monde remplissant un certain nombre de conditions. Elles évoquent « la clause tout État $»{ }^{31}$ En vertu de celle-ci, lorganisation internationale reste ouverte à tous les États remplissant les conditions posées par l'acte constitutif. Nous pouvons citer comme exemples dorganisations universelles l'ONU, le FMI, l'UNESCO, l'OIT, etc. Quant aux organisations régionales, ce sont celles qui sont réservées exclusivement aux États liés par une solidarité déterminée, qui peut être géographique (Union Africaine, Conseil de l'Europe, Organisation des États d'Amérique), linguistique et ethnique (Ligue des États Arabes), religieuse (Organisation de la Conférence Islamique), politique et militaire (OTAN). Relativement à la présente thèse, ce critère permet justement de saisir les conditions de composition des organes restreints. De fait, dans les organes restreints des organisations universelles ces critères vont témoigner des clivages économiques et politiques qui existent entre les différents groupes d'États membres. En revanche, dans les organisations régionales, en raison de la solidarité régionale, la tendance sera portée sur lélimination des discriminations relatives aux critères de sélection des membres des organes restreints.

Avec le critère de l'objet, les organisations internationales sont classées en organisations à vocation générale et en organisations spécialisées. Les organisations à vocation générale sont celles qui ont des objectifs généraux et dont lobjet embrasse tous les domaines des relations

KPODAR (Adama), "Le principe de spécialité dans la définition des organisations internationales », RBSJA, $\mathrm{n}^{\circ} 17$, Décembre 2006, p.55.

COMBACAU (Jean) et SUR (Serge), Droit international public, op.cit., p.709.

BEN ACHOUR (Rafaâ), Institutions de la société internationale, Centre de publication universitaire, Tunis 2004, p.160.

NGUYEN QUOC (Dinh), DAILLIER (PATRICK), FORTEAU (Mathias), PELLET (Alain), Droit international public, op.cit., p.193. 
internationales : domaines politique, économique, social, culturel, technique, scientifique, humanitaire, etc.

L'ONU, l'UA, l'OEA sont des organisations internationales à vocation générale. Les organisations spécialisées portent sur un objet limité et dont l’activité ne concerne qu’un domaine déterminé. Certaines de ces organisations ont un objet économique (FMI, Banque mondiale, etc). D’autres ont un objet social (OIT, OMS, etc.). D’autres encore ont un objet technique (UPU, OACI...). Le recours à un tel critère dans cette thèse aura l'avantage de mettre en relief l'attitude des membres des organes restreints vis-à-vis des privilèges fonctionnels accordés à certains États. Dans les organisations à vocation générale, on observe la propension de certains États, surtout des États en voie de développement, à contester les privilèges fonctionnels reconnus aux grandes puissances, au nom du principe de l'égalité juridique. Par contre, dans les organisations spécialisées, ces contestations sont amoindries en raison du fait que les critères de sélection des membres des organes restreints se fondent sur des valeurs purement techniques et économiques.

Il y a enfin le critère des pouvoirs. Sur la base de ce critère, on distingue les organisations d'intégration des organisations de coopération. Les organisations d'intégration sont des organisations qui tendent vers la fédération. Dans celles-ci, les États membres opèrent au profit de l'organisation un transfert de compétences. Elles sont en principe dotées de pouvoirs qui limitent ceux des États membres. Tel est le cas de l'Union Européenne. Quant aux organisations de coopération, elles visent à coordonner les positions et à prendre des mesures communes aux États-membres. À titre d'exemple, nous pouvons citer l'UA, l'ONUDI, l'UNESCO, etc. ${ }^{32}$ Cette classification donne une orientation sur le degré de souveraineté des membres des organes restreints. Dans les organisations d'intégration, en raison du transfert des compétences des États membres à lorganisation, les organes restreints ne seront pas toujours soucieux du respect de la souveraineté de ses membres. À l'opposé, dans une organisation de coopération, les organes restreints remettent en cause difficilement cette souveraineté des États membres. Mais qu'est-ce qu’un organe restreint?

\section{B : LA NOTION D'ORGANES RESTREINTS}

Pour cerner juridiquement la notion d'organes restreints, il est important de mettre en lumière la diversité des organes des organisations internationales (1), puis de montrer le particularisme des organes restreints dans la structure organique de celles-ci (2).

32 Cette classification a été proposée par le Professeur Rafâa BEN ACHOUR. BEN ACHOUR (Rafaâ), Institutions de la société internationale, Centre de publication universitaire, Tunis 2004, p.160-162. 


\section{1) LA DIVERSITE DES ORGANES DES ORGANISATIONS INTERNATIONALES}

Les États qui établissent une organisation internationale sont obligés de mettre en place des organes qui expriment leur intention détablir une institution permanente distincte de ses membres. Le Professeur DIEZ DE VELASCO affirme que « les organes incarnent par leur structure institutionnelle la continuité et la stabilité de l'organisation, en même temps qu'ils reflètent son indépendance à l'égard des États membres.» ${ }^{33}$

Il existe une variété d’organes aux noms et aux fonctions très multiples. Cependant, la doctrine a identifié une série de critères qui permettent de les classer. Le Professeur NGUYEN QUOC Dinh se fonde sur trois critères pour catégoriser les organes des organisations internationales. Il s'agit des critères tenant à l'origine, à la composition et aux fonctions des organes.

Le critère tenant à lorigine, nous perment de distinguer les organes originaires qui procèdent directement du traité constitutif de l'organisation et les organes dérivés ou subsidiaires qui sont créés par les organes originaires en vertu des pouvoirs que leur reconnait le traité constitutif. L’Assemblée générale, le Conseil de sécurité, le Conseil économique et social, entre autres, constituent des organes originaires de l'ONU. ${ }^{34}$ En revanche, la CNUCED, le PNUD, le HCR sont des organes subsidiaires de l'Assemblée générale des Nations Unies. Dans le cadre de cette thèse, nous nous intéresserons essentiellement aux organes originaires des organisations internationales.

Il faut aussi mentionner le critère des fonctions.On distingue les organes politiques des organes administratifs. Si les organes politiques définissent la politique générale de l’organisation, au contraire, les organes administratifs se chargent de la préparation, de l'exécution des décisions de l'organisation. Dans la catégorie des organes politiques peuvent être rangés l’Assemblée générale et le Conseil de sécurité de l'ONU, la Conférence des Chefs d'État et de gouvernement de l'UA. En revanche, font partie des organes administratifs, le Secrétariat général de l'ONU, la Commission de l'UA. Dans cette classification fondée sur le critère de la fonction, les organes juridictionnels méritent d’être relevés. En guise d'exemples, nous avons le TANU, la CIJ, le TAOIT. Dans le cadre de notre analyse, cette classification nous situe sur la répartition des pouvoirs entre les différents organes des organisations internationales.

Une classification importante doit être aussi relevée. C'est celle qui se fonde sur la composition des organes. De ce point de vue, deux catégories d’organes peuvent être étudiées. D’une part, les organes pléniers qui comprennent les représentants de tous les membres de l'organisation. Il s’agit des organes comme l'Assemblée générale de l'ONU, la Conférence des Chefs d'État et de gouvernement de l'UA, etc. D'autre part, les organes restreints qui regroupent un nombre limité de membres. Nous pouvons y inscrire les organes comme le Conseil de sécurité, le Conseil économique et social, le Conseil de Tutelle de l'ONU, la Commission de l'UA, la Commission de l'UE, le Conseil

DIEZ DE VELASCO VALLEJO (Manuel), Les organisations internationales, Paris, Economica, 2002, p.13. NGUYEN QUOC (Dinh), DAILLIER (Patrick), FORTEAU (Mathias), PELLET (Alain), Droit international public, op.cit., p.681-688. 
d’administration de l'OIT, le Conseil d'administration du FMI, le Conseil de l'Autorité des fonds marins, le Conseil de paix et de sécurité de l'UA, etc. C’est cette dernière catégorie qui recèle des particularités importantes au sein des organisations internationales que portera notre réflexion.

\section{2) LE PARTICULARISME DES ORGANES RESTREINTS DANS LA STRUCTURE DES ORGANISATIONS INTERNATIONALES}

Le particularisme des organes restreints dans la structure des organisations internationales découle surtout du nombre limité des membres qui les composent. De façon générale, la raison dêtre d'une organisation internationale, comme de toute institution, est fonctionnelle. C'est la volonté, chez ses fondateurs de disposer d'un instrument propre à la poursuite d’objectifs définis qui explique la création d'une organisation internationale. C’est la nature des activités nécessaires à la réalisation de cette fonction qui détermine la structure organique dont cette organisation est dotée par son acte constitutif. Il existe donc une relation dialectique entre les fonctions de lorganisation et ses éléments structurels. ${ }^{35} \mathrm{La}$ création d'un organe restreint au sein d'une organisation internationale n'est pas fortuite.

Dans les organisations internationales qui comptent plusieurs membres, il n'est pratiquement pas possible de délibérer sur tous les points en Assemblée plénière. Les traités constitutifs prévoient dans la plupart des cas des organes restreints constitués par un nombre limité de membres et dotés d’attributions plus ou moins importantes. Le but poursuivi par le traité constitutif en créant des organes restreints est fonctionnel. Les organes restreints permettent le fonctionnement régulier de l'organisation.

Toutefois, au-delà de l'aspect fonctionnel, la question de l'égalité entre les États dans les organisations internationales apparaît dans les organes restreints. En principe chaque État membre d'une organisation internationale possède un siège au sein de lorganisation, et par conséquent une voix. ${ }^{36}$ En principe tous les suffrages sont égaux. ${ }^{37}$ Toutefois, le principe égalitaire connaît des exceptions dues à la nécessité d'introduire diverses formes d'inégalité dans la représentation au sein des organes restreints. Cette inégalité tient d’abord à une exigence structurelle impérative : le nombre limité de membres et la périodicité des organes pléniers. Elle tient ensuite, et surtout, à la nécessité politique d'assurer la participation quasi-automatique et effective d'un certain nombre d’États membres qui se distinguent par leurs qualités objectives particulières.

\footnotetext{
35 VIRALLY (Michel), L'organisation mondiale, Collection U, Paris, Armand Colin, 1972, p.26.

36 AUDOIN (Jean- Pierre), L'Autorité internationale des fonds marins, (d’après les travaux de la troisième Conférence des Nations Unies sur le droit de la mer), Thèse pour le doctorat d'Etat en droit, Nice, Septembre, 1979, p.181. 
Les organes restreints se singularisent par ses deux traits qui conditionnent leur création : l'efficacité de l'organisation internationale et l'inégalité entre les États. Et, justement la problématique de cette étude découlera de ces deux points.

\section{III/ LA PROBLEMATISATION DU SUJET}

Selon Michel BEAUD, la problématique se définit comme «l'ensemble construit, autour d'une question principale, des hypothèses de recherche et des lignes d'analyse qui permettront de traiter le sujet choisi ${ }^{38}$ La problématisation du sujet consistera à faire ressortir la question centrale qui en découle. De ce fait, nous verrons le problème juridique que soulève notre sujet (A) et tenterons d’expliciter les éléments clés de cette problématique (B).

\section{A : LA POSITION DU PROBLEME}

De façon générale, les États créent les organisations internationales en vue de juguler ensemble les problèmes qui se posent à eux dans la vie internationale. Les organisations internationales ont un but essentiellement fonctionnel. Aussi, les organes qui les composent, particulièrement les organes restreints, s'inscrivent-ils dans cette logique fonctionnelle. Ceux-ci sont institués en vue de rechercher l'efficacité des organisations internationales. Le nombre limité des membres qui composent ces organes leur permet de répondre rapidement aux problèmes qui se posent à l’ensemble des États membres siégeant dans les organes pléniers.

Mais, la composition réduite des organes restreints soulève inéluctablement des questions au sein des organisations internationales. Ces problèmes tournent autour de l'égalité des membres dans les organes restreints : les conditions d'accès, la composition et le statut des États-membres au sein de ces organes, etc.

C'est donc logiquement que la problématique de notre sujet va se construire autour des deux notions essentielles que sont l'efficacité de lorganisation internationale et légalité dans les organes restreints.

La question fondamentale à laquelle nous tenterons de répondre est celle de savoir si l'efficacité recherchée par les États membres en créant des organes restreints favorise la mise en œuvre du principe d'égalité dans les organisations internationales? Autrement dit, la recherche de l'efficacité recherchée à travers les organes restreints ne compromet-elle pas le principe de l'égalité juridique des États membres de l'organisation internationale?

En vue de mieux comprendre cette question, il s'avère nécessaire de clarifier les éléments essentiels qui la composent. 


\section{B : LA CLARIFICATION DES ELEMENTS DE LA PROBLEMATIQUE}

Il est nécessaire de préciser le contenu de la notion d'efficacité dans les organisations internationales (1), puis d'envisager le point touchant au respect du principe dégalité dans les organisations (2).

\section{1) LA NOTION D’EFFICACITE DANS LES ORGANISATIONS INTERNATIONALES}

L'efficacité d'une organisation internationale doit s'appréhender avant tout par rapport à son but. L'organisation internationale exprime un besoin ressenti par les États membres : celui de s'unir en vue d'atteindre des objectifs communs. Par définition toutes les organisations ont des missions qui impliquent une capacité d'action autonome dans les relations internationales. ${ }^{39}$

C’est au regard de ses missions que l'efficacité d'une organisation internationale doit être évaluée. Elle doit atteindre les objectifs qui ont été assignés par les États membres. La structure organique des organisations internationales doit être nécessairement orientée dans ce sens. L'existence d'organes répond au besoin d'une structure interne articulée de l'organisation internationale. ${ }^{40}$ À cet effet, l'acte constitutif attribue des compétences spécifiques à chaque organe. ${ }^{41}$ La répartition des compétences entre les différents organes s'inscrit logiquement dans une finalité fonctionnelle.

Mais pour apprécier l'efficacité des organes restreints, il est nécessaire de rechercher leurs contributions dans le fonctionnement des organisations internationales. La contribution des organes restreints dans le fonctionnement des organisations internationales fait absolument appel à des critères d'appréciation rigoureux. Elle peut se faire à un double point de vue.

Le premier niveau dévaluation de l'apport des organes restreints porte sur le fonctionnement interne des organisations internationales. Les organisations internationales exercent une fonction exécutive que l'on appelle aussi fonction de gouvernement et même fonction d'administration. ${ }^{42}$ Les organes restreints semblent être les mieux placés pour permettre aux organisations internationales de mettre en œuvre cette fonction. Ce constat résulte d'une analyse des actes constitutifs des organisations internationales et des règlements intérieurs des organes restreints étudiés. Ces textes nous permettront de vérifier si les organes restreints favorisent la réalisation des missions assignées aux organisations internationales.

39 KPODAR (Adama), "Le principe de spécialité dans la définition des organisations internationales », op.cit., p.57.

$40 \quad$ Ibid., p.55.

41 Ibid.

42 MONACO (Riccardo), « Les principes régissant la structure et le fonctionnement des organisations internationales », op.cit., p.149. 
Le second niveau d'évaluation de l'efficacité des organes restreints peut aussi se mesurer en rapport avec l'activité extérieure des organisations internationales. De façon précise, les relations entre les organisations internationales et les Etats constitueront une grande partie des réflexions. Dans cette perspective, l'analyse de la portée réelle des pouvoirs reconnus à ces organes par les actes constitutifs sera utile pour cerner un autre aspect de l'efficacité des organisations internationales dans l'ordre internationale. Il s'agira de chercher à clarifier le contenu des compétences attribuées aux organes restreints et leurs effets dans les rapports qu'ils entretiennent avec les États.

La quête de l'efficacité de l'organisation internationale constitue une des raisons qui justifie la création des organes restreints. Mais sa réalisation dépend du respect du principe dégalité des États au sein de ces organes.

\section{2) LE RESPECT DU PRINCIPE D’EGALITE DES ÉTATS DANS LES ORGANES RESTREINTS}

Le principe d'égalité des États est une règle fondamentale du droit international. On distingue généralement dans la doctrine l'égalité devant la loi (equality before the law) et légalité des droits (equality of rights). ${ }^{43}$ L'égalité devant la loi est une notion qui relève du domaine d'application de la loi. Elle signifie que la loi doit être appliquée sans tenir compte des considérations extralégales. En droit international, les États ont tous le devoir de respecter de bonne foi les engagements qu'ils prennent et assument le même type de responsabilité pour toute violation de ce droit. Par contre, l'égalité des droits signifie que dans un domaine donné un système juridique confère les mêmes droits et obligations à tous ou à certains sujets. Cette notion relève du contenu du droit. ${ }^{44}$ Les Professeurs Pierre-Marie DUPUY et Yann KERBRAT soulignent que le principe dégalité «signifie que tous les Etats se trouvent placés devant le droit dans une situation identique.Celui-ci leur reconnaît à priori les mêmes aptitudes légales, les mêmes droits et les mêmes obligations " ${ }^{45}$

Si dans les organes pléniers le principe d'égalité est respecté, en revanche dans les organes restreints, son application présente beaucoup de difficultés. Quels sont donc les problèmes qu'il soulève dans ces organes et quels en sont les implications?

Les organes restreints ne sont pas ouverts à tous les États membres de l'organisation. C'est seulement à une catégorie d'États privilégiés qu'ils sont réservés. Dans ces organes, les grandes puissances jouissent de privilèges fonctionnels exorbitants. De ce fait, apparaît une inégalité fonctionnelle entre les États qui participent aux travaux de ces organes restreints. ${ }^{46}$

À première vue, la raison dêtre de l'organisation internationale est la consécration du principe de légalité entre les États. ${ }^{47}$ Ce principe en droit international signifie que les États sont

KRANZ (Jerzy), « Le vote pondéré dans les organisations internationales », RGDIP, 1981, p.334. Ibidem.

DUPUY (Pierre-Marie), KERBRAT (Yann), Droit international public, $11^{\mathrm{e}}$ édition, Dalloz, Paris, 2012 , p.119. MERLE (Marcel), La vie internationale, op. cit., p.62 et suivants. BOUTROS-GHALI (Boutros), « Le principe dégalité des États et les organisations internationales », op.cit.p.30. 
tous souverains ; subséquemment, ils ont tous les mêmes droits et obligations. De ce point de vue, les États, sujets primaires et originaires du droit international public, devraient normalement siéger sur un pied dégalité dans les organes restreints des organisations internationales. Ce constat suscite des interrogations majeures. L'État, citoyen de l'organisation, ${ }^{48} \mathrm{y}$ accède au même titre que tous les autres États. Il en résulte que tous les organes de l’organisation devraient être ouverts sans aucune discrimination à tous les États.

Au regard du principe d'égalité des États, qu'est-ce qui fonde juridiquement les inégalités entre les États qui participent aux organes restreints des organisations internationales?

Selon BOUTROS-GHALI, « lorganisation internationale vise essentiellement à mettre en œuvre l'égalité des États, mais il est nécessaire d’accorder aux grandes puissances certaines prérogatives pour tenir compte des responsabilités accrues qui leur incombent et du pouvoir réel dont elles disposent. C'est essayer de concilier l'inconciliable.» ${ }^{49}$

Il existe de très grandes différences de vulnérabilités entre les États, suivant leurs situations géostratégiques et le degré de leur stabilité ou instabilité interne et dans les moyens de puissance acquis. ${ }^{50}$ Mais, ce sont ces États qui sont appelés à siéger dans les organes restreints des organisations internationales. Cette représentation des États dans les organes restreints ne devrait pas soulever de problème au regard du principe dégalité devant le droit international. Les États, dans leur diversité, sont tous égaux devant le droit international. Cette égalité des États est formellement proclamée par les Chartes constitutives des organisations internationales. La Charte de l'ONU en son article 2 paragraphe 1 dispose que "l’organisation est fondée sur le principe de légalité souveraine de tous ses membres. $\rangle^{51}$ Quant à l'Acte constitutif de l'Union Africaine, il prévoit en son article 4, paragraphe (a) « l'égalité souveraine et l'interdépendance de tous les membres de l'Union ».52

Les Etats devraient sièger dans les organes restreints sur la base du principe d'égalité. Toutefois, le problème apparaît au niveau des privilèges fonctionnels accordés aux grandes puissances dans ces organes. Les Etats doivent-ils jouir des mêmes privilèges dans les organes restreints en dépit de leurs inégalités matérielles ? Cette question pertinente nous amène à analyser les implications du principe dégalité dans les organes restreints des organisations internationales. Au plan juridique, la question qui mérite dêtre posée est celle relative à la signification du principe d’égalité des États dans les organisations internationales ? En effet, pour BOUTROS-GHALI, « dans le cadre du droit des organisations internationales, le principe d'égalité des États se présente de trois manières différentes.»

\footnotetext{
48 DUPUY (René-Jean), La communauté internationale, entre le mythe et l'histoire, op.cit., p.56.

49 BOUTROS-GHALI (Boutros), "Le principe dégalité des États et les organisations internationales ", op.cit., p.30.

$50 \quad$ Ibid, p.40 et 41.

51 Voir l'article 2 paragraphe 1 de la Charte des Nations Unies

52 Voir l'article 4 paragraphe (a) de l'Acte constitutif de l'Union Africaine.
} 
La première approche du principe d'égalité des États est celle qui postule l'égalité matérielle des États membres de l’organisation internationale. Conformément à ce principe, l'organisation internationale ne devrait comprendre que des membres égaux, réellement et juridiquement, parce qu'il n’y a pas dégalité juridique sans un minimum dégalité matérielle. Cette conception de légalité est difficilement réalisable. Lévolution des diverses organisations internationales révèle qu’en dépit des principes égalitaires qu'elles proclament, la tendance de l'un des États membres ou d'un groupe restreint d'États à assurer la direction politique de l'organisation. ${ }^{53}$

La seconde approche est celle de l'égalité absolue ou fonctionnelle des États membres de lorganisation internationale. ${ }^{54}$ Tous les États sont égaux quelle que soit la manière dont ils sont devenus membres de l'organisation, et l'idée d'une participation limitée ou de l'admission d'une catégorie spéciale de membres jouissant de droits restreints est exclue. Enfin, tous les États doivent se faire représenter dans tous les organes de l'organisation, dans lesquels ils ont tous droit au même nombre de délégués. ${ }^{55}$ Cette approche du principe dégalité des États est celle qui prévaut dans certaines organisations internationales régionales telle que l'UA. Si elle a le mérite de réaliser la mise en œuvre de ce principe, en revanche, elle soulève le problème de l'efficacité de l'organisation internationale. Mais, lorganisation internationale peut-elle fonctionner efficacement si tous les États sont mis sur un pied d'égalité fonctionnelle? En d'autres termes, ne faut-il pas accorder des privilèges fonctionnels aux grands États en compensation de leurs contributions financières considérables?

La dernière conception de légalité des États dans les organisations internationales est celle qui prône l'égalité relative ou l'inégalité fonctionnelle. Elle tend à corriger les excès de légalitarisme et à rétablir l'équilibre entre l’inégalité de fait et l'égalité de droit, entre le respect des responsabilités et la loi du nombre. Ici, l'égalité juridique ne signifie ni égalité matérielle, ni égalité de participation aux fonctions internationales, mais égalité devant la loi internationale. C’est la formule adoptée par l'ONU. Les grands États bénéficient d'une situation privilégiée (sièges permanents, pouvoir de vote spécial) en raison de leurs responsabilités dans la société inter-étatique.

Deux exigences contradictoires sous-tendent la mise en place des organes restreints dans les organisations internationales. Il s'agit d’appliquer le principe d’égalité entre les États membres des organes restreints. Cette application du principe dégalité revendiquée par les petits États implique nécessairement la suppression des privilèges fonctionnels des grandes puissances. Ce sont, entre autres, le droit de véto, le statut de membres permanents et une certaine pratique du consensus qui est détournée de sa vocation initiale..$^{56}$ Invoquant l'efficacité de l'organisation et leurs contributions matérielles au fonctionnement de celle-ci, les grandes puissances semblent sopposer à toute démocratisation des organes restreints.

Ibid, p.18.

Ibid, p. 15

Ibid, p.22.

MAHIOU (Ahmed), "L'Algérie et les organisations internationales », AFDI, 1982, p.138. 
Une fois encore, le principe de la démocratie politique et le principe de la démocratie économique et sociale vont s'affronter dans une tension dialectique que les États entretiennent par ailleurs assez volontiers dans le cadre des organisations internationales. ${ }^{57} \mathrm{La}$ démocratie politique évoque la loi du nombre dans les organisations internationales. Elle consiste à tenir compte de la majorité des États dans la distribution des privilèges fonctionnels. Au contraire, la démocratie économique se fonde sur le poids des contributions des États dans la répartition des sièges et des pouvoirs politiques.

La confrontation de l'efficacité de l'organisation et légalité des États constitue la question centrale qui sera analysée dans la présente thèse. À cet effet, il est important de préciser l'intérêt et la méthodologie qui guideront notre réflexion.

\section{III/ LA PRECISION DE L'INTERET DU SUJET ET DE L'APPROCHE METHODOLOGIQUE}

Il est important de mettre en lumière l'intérêt de cette étude, d'une part (A), et la démarche méthodologique que nous utiliserons dans nos analyses, d’autre part (B).

\section{A : L'INTERET DU SUJET}

L'intérêt de notre sujet se situe à un double niveau. Au plan scientifique, les réflexions qui seront menées constituent une contribution à létude du droit des organisations internationales (1). Mais, au-delà de la matière, ce sujet nous permet de jeter un regard critique sur les rapports politiques entre les grandes puissances et les petits États au sein de ces organes (2).

\section{1) LA CONTRIBUTION À L'ETUDE DU DROIT DES ORGANISATIONS INTERNATIONALES}

Lavènement des organisations internationales dans les relations internationales a enrichi l'étude du droit international public. En leur qualité de sujet de droit international, elles ont contribué à lémergence d'un droit particulier : le droit des organisations internationales.

La définition de ce droit fait l'objet de controverses doctrinales. Le Professeur NGUYEN QUOC Dinh souligne que « certains auteurs soutiennent qu'il faut distinguer le droit applicable aux organisations de celui sécrété par les organisations internationales. Le premier seul relèverait du droit international, le second, souvent dénommé droit interne des organisations internationales, constituant un droit distinct, trop différent des caractéristiques du droit international général pour s'y incorporer $\gg{ }^{58}$ Mais, cette conception a des limites. Le droit sécrété par les organisations p.540-541.

58 NGUYEN QUOC (Dinh), DAILLIER (Patrick), PELLET (Alain), FORTEAU (Mathias), Droit international public, op.cit., p.641. 
internationales tire sa source des chartes constitutives qui sont des traités multilatéraux, sources classiques du droit international. Les Professeurs Jean COMBACAU et Serge SUR estiment « quà première vue, il y a une diversité du droit des organisations internationales dans la mesure où chaque organisation est avant tout régie par son instrument de base, constitué par un traité interétatique. Il existe donc un principe de spécificité des organisations internationales». ${ }^{59}$ Toutefois, ils précisent que « certains éléments d'un droit commun des organisations internationales peuvent cependant être dégagés [...] Ce droit commun est supplétif, puisqu’il n’est destiné à s’appliquer que dans le silence du traité constitutif [...] Il existe également des pratiques communes aux différentes organisations $»{ }^{60}$

En dépit de ces approches multiples, le droit des organisations internationales peut se définir comme l’ensemble des règles juridiques qui président à la naissance de lorganisation internationale et qui régissent son fonctionnement et ses activités. Il s'agit du droit applicable aux organisations internationales et de celui qu'elles sécrètent : le traité de base qui crée l'organisation, l'accord de siège, les traités conclus avec les sujets de droit international, le règlement intérieur des divers organes, les actes individuels, les actes collectifs ayant trait aux agents, à l'administration. ${ }^{61}$

Cette thèse constitue une réflexion scientifique sur le droit des organisations internationales. A partir de la notion dorganes restreints nous examineront les principes et règles fondamentales qui régissent les organisations internationales. De ce fait, nous serons conduits à analyser au plan juridique la notion d'organisations internationales.

La création d'une organisation internationale obéit surtout à des considérations d’ordre fonctionnel. Les actions isolées des États ne peuvent suffire à la satisfaction complète de leurs besoins. Le recours à l'organisation internationale, en tant que moyen d'action, amène les États à s'associer à un moment donné du développement de leurs relations pour atteindre des objectifs identiques. ${ }^{62}$ Dans cette perspective, les organisations internationales sont tenues d'atteindre les résultats escomptés par les États membres. La création d’un organe restreint doit s'inscrire dans la recherche de l'efficacité des organisations internationales. À travers cette thèse, nous évaluerons le rôle des organes restreints dans le fonctionnement des organisations internationales. Mais, au plan politique elle permettra d'analyser les rapports de force entre les États membres dans les organisations internationales.

COMBACAU (Jean), SUR (Serge), Droit international public, op.cit., p.708. Les deux auteurs soulignent que ce principe de spécificité des organisations internationales "correspond à leur caractère instrumental, puisque à chacune d'elles sont dévolues une ou des tâches particulières, qui commandent leur structure comme létendue de leurs compétences et pouvoirs. Spécificité d’autant plus forte que chaque organisation va développer sa propre régulation interne qui, en accroissant la densité de sa réglementation autonome, léloignera des autres".

$60 \quad$ Ibid.

61 YAO-N'DRÉ (Paul), Droit des organisations internationales, Abidjan, PUCI, 1996, p.35.

$62 \quad$ Ibid, p.33. 


\section{2) LA MISE EN EVIDENCE DES RAPPORTS DE FORCE ENTRE LES ÉTATS MEMBRES DANS LES ORGANES RESTREINTS}

Un fait majeur mérite dêtre souligné : les organisations internationales, bien qu'elles répondent à un besoin collectif ressenti par ses fondateurs, ne sont pas des organisations créées à des fins philanthropiques ou caritatives. Bien au contraire, les « inégalités de puissance " ${ }^{63}$ entre les États membres de la société internationale ont des répercussions dans les organes restreints des organisations internationales. Elles se perçoivent généralement dans les rapports de force entre les grandes puissances et les petits États.

C'est à juste titre que l'un des points essentiels de notre réflexion sera la mise en évidence de la nature de ces rapports de force. Ce point est important dans la mesure où une fois qu'une organisation internationale est mise en place, les groupes d'intérêt cherchent à affirmer leur prépondérance. ${ }^{64} \mathrm{La}$ dynamique des organisations internationales enseigne que les forces politiques en présence s'y affrontent constamment, travaillant à transformer un équilibre constitutionnel toujours précaire afin d'assurer la suprématie réelle de l’organe qu'elles pensent dominer. ${ }^{65}$ Les États membres des organisations internationales ont naturellement bien saisi cette relation étroite entre la place et la composition de l'organe restreint et les pouvoirs réels dont celui-ci disposera. ${ }^{66}$

C'est au sujet de la place des organes restreints dans la structure organique des organisations internationales que les petits États et les grandes puissances s’affrontent avec le plus de véhémence. La première opposition entre les pays en voie de développement et les pays développés se situent au niveau de la question délicate des rapports à établir entre les organes pléniers et les organes restreints. On doit également souligner bon nombre de divergences relatives à la composition des organes restreints dans la mesure où elle s'accompagne obligatoirement d'un choix particulier quant aux modalités de prises de décision.

$\mathrm{Au}$ demeurant, cette tension entre les petits États et les grandes puissances sur la question de la place des organes restreints dans les organisations internationales met en relief la confrontation de la démocratie politique et de la démocratie économique et sociale. ${ }^{67}$ L'intérêt du sujet étant précisé, il est opportun de souligner la méthode qui guidera nos analyses.

\footnotetext{
63 VIRALLY (Michel), "Cours général de droit international public », RCADI, 1983-V, Tome 183, p.40.

64 AUDOIN (Jean-Pierre), L'Autorité internationale des fonds marins (D’après les travaux de la troisième conférence des Nations Unies sur le droit de la mer), op.cit., p.165.

$65 \quad$ Ibid.

66 Ibid.

67 DUPUY (René-Jean), « L’Organisation internationale et la volonté générale », RGDIP, 1957, p.540-541.
} 


\section{B: L'APPROCHE METHODOLOGIQUE DU SUJET}

Toute étude scientifique implique nécessairement le choix d'une méthode. Selon Madeleine GRAWITZ la méthode est l' " ensemble de procédures permettant d’obtenir à propos d'un objet quelconque, une théorie située à un niveau de vérification aussi élevé que possible et permettant d'expliquer l'interdépendance des éléments constitutifs de cet objet $"{ }^{68}$ Loption de la démarche dialectique semble convenir à cette étude (1). Pour mieux clarifier cette approche méthodologique, il est primordial de procéder à une délimitation du sujet (2).

\section{1) L'OPTION DE LA METHODE DIALECTIQUE}

Pour cerner un objet au plan scientifique, plusieurs méthodes s'offrent à la réflexion. Madeleine GRAWITZ procède à une énumération de ces méthodes. ${ }^{69} \mathrm{Il}$ y a la méthode historique qui s’appuie sur un temps, peut être artificiellement, reconstruit, mais assurant une continuité, une trame aux phénomènes. La méthode génétique, qui elle, cherche la genèse des évènements, c'est-àdire les antécédents. La méthode fonctionnelle, quant à elle, se fonde sur l'idée de fonction. Celle-ci implique tout simplement la façon dont une institution fonctionne dans le système social auquel elle appartient. La méthode systémique a pour but de construire un modèle ou un cadre théorique à l'analyse du système socioculturel. Enfin, la méthode dialectique qui part de la constatation très simple des contradictions qui nous entourent.

De toutes ces méthodes de recherche, Madeleine GRAWITZ soutient que « la méthode dialectique est plus complète que les autres [...] car elle correspond aux exigences fondamentales de la notion même de méthode ». C’est cette raison qui justifie le choix de cette méthode dans le cadre de notre thèse.

Le Professeur René-Jean DUPUY définit la dialectique comme « une méthode qui se fonde sur l'étude des contradictions et l'affrontement des tendances opposées, elle fournit des expériences très variées grâce au contact qu’elle permet avec les sinuosités du réel; c'est elle-même une méthode de lutte contre toute cristallisation; elle tend à la mise en relief des contradictions et des complexités toujours renouvelées, de l'ensemble du phénomène social étudié.» ${ }^{70}$

Cette méthode a le mérite de confronter les données théoriques à la réalité. Or, dans le cadre de cette thèse, les informations que nous avons des organes restreints procèdent de leurs règlements intérieurs et des actes constitutifs des organisations internationales. L'exégèse de ces textes nous permettra de saisir des aspects fondamentaux du rôle de ces organes au sein des organisations internationales. Mais il faut le souligner, l'interprétation de ces textes ne suffit pas, à elle seule, à cerner toutes les dimensions de l'institution de ces organes dans les organisations 
internationales. Le juriste ne doit pas limiter sa tâche à la connaissance de la forme pure du droit ; il doit aussi connaitre et interpréter les faits qui ont conditionné la naissance de telle ou telle norme juridique et qui en accompagnent l'application. ${ }^{71}$ Pour cerner la question des organes restreints des organisations internationales, il est tout à fait opportun de combiner la méthode des écoles positivistes ou normativistes fondée sur une étude exégétique des dispositions textuelles avec la méthode sociologique qui prend en compte les motivations politiques et idéologiques qui soustendent la mise en place des institutions internationales.

Cette démarche méthodologique ne saurait être achevée si nous ne procédons pas à une délimitation scientifique du sujet.

\section{2) LA DELIMITATION DU SUJET}

Traiter un sujet portant sur les « organes restreints des organisations internationales » est une véritable gageure. Évidemment, une telle étude présente des difficultés au plan scientifique. Celles-ci proviennent principalement du nombre très élevé des organisations internationales, et elles occupent dans lordre international une place considérable. ${ }^{72} \mathrm{Il}$ est donc impossible de les étudier dans le cadre d'une thèse. En plus, chaque organe restreint d'une oganisation internationale a sa spécificité.

Pour ces raisons, nous avons jugé utile de sélectionner quelques organes restreints dorganisations internationales, qui feront l'objet de notre réflexion. Nous sommes conscients que toute délimitation d'un sujet comporte un certain arbitraire et nous ne prétendons pas y échapper. ${ }^{73}$

Mais, les organes restreints qui constitueront l'objet de cette thèse ont la particularité de relever les problèmes communs soulevés par ceux-ci au sein des organisations internationales. Sur la base de la classification des organisations internationales opérée ci-dessus, quatre types d’organisations internationales seront analysées : l'ONU qui est une organisation universelle à vocation politique, le FMI et l'Autorité internationale des fonds marins qui sont aussi des organisations internationales universelles mais qui ont une finalité technique et l'UA qui est une organisation régionale de coopération..$^{74}$

71 MIELE (Mario), «Les organisations internationales et le domaine constitutionnel des États », RCADI, 1970-III, p.322.

72 DUPUY (René-Jean), « Le droit des relations entre les organisations internationales », RCADI, 1960-II, P.462

$73 \quad$ Ibid, p.465.

74 Comme on peut le constater, dans le champ de notre étude figurent presque tous les critères de classification des organisations internationales. Le critère de la composition des organisations internationales prend en compte la distinction organisations universelles (ONU, FMI, Autorité internationale des fonds marins) et organisations régionales (UA); celui de la fonction est relatif à la distinction organisations à vocation politique $(\mathrm{ONU})$, organisations à caractère technique (FMI, Autorité internationale des fonds marins) et organisations de coopération (UA) ; celui des pouvoirs fait la distinction entre les organisations politiques (ONU) et les organisations de coopération (UA). 
Le choix des organisations internationales qui seront analysées dans notre thèse étant fait, il est important de procéder à la désignation des différents organes restreints. Nous mentionnerons les organes restreints des organisations internationales à vocation universelles avant les organes restreints des organisations régionales. Suivant cet ordre, sept organes restreints seront retenus.

Dans le cadre de l'ONU, deux organes restreints feront l'objet de nos analyses. Au plan historique, l'ONU est née de la Conférence de San Francisco (Etats-Unis) qui a eu lieu du 25 avril au 16 juin 1945. Elle a succédé à la SDN. Sa Charte a été signée le 26 juin 1945. Elle comprend 6 organes principaux. Parmi ceux-ci, nous nous intéresserons au Conseil de sécurité et au Conseil économique et social. Le Conseil de sécurité est un organe principal autonome de l'ONU dont la mission principale est le maintien de la paix et de la sécurité internationales. ${ }^{75}$ Dans sa composition, il comprend 15 membres dont cinq sont permanents, à savoir la Chine, les États-Unis d’Amérique, la Fédération de Russie, la France et le Royaume-Uni. Quant aux 10 autres membres, ils sont élus pour une période de deux ans - et ne sont pas immédiatement rééligibles - par l'Assemblée générale qui tient compte de leur contribution « au maintien de la paix ainsi que «d'une répartition géographique équitable $» \cdot{ }^{76}$ Le choix de cet organe restreint se justifie par sa particularité dans le système de l'ONU. Étant l'organe ayant «la responsabilité principale du maintien de la paix et de la sécurité internationales ", ses pouvoirs se confondent presqu'avec ceux de l'organisation. Son fonctionnement, marqué par les pouvoirs de vote spéciaux des grandes puissances suscite une réflexion approfondie.

Le Conseil économique et social constitue aussi un organe principal mais non autonome de l'ONU. ${ }^{77}$ L'idée d'un organe « d'initiative et de coordination » capable de jouer un rôle en matière économique et sociale date de la SDN. À mesure que se développait ce domaine d’activité et que son importance était mieux comprise, diverses propositions ont visé la création d'un organe spécialement habilité en la matière. Mais, ce n’est qu’en mai 1939 que des mesures précises ont été prises dans le cadre de la SDN. ${ }^{78}$ Cette expérience a ouvert la voie aux rédacteurs de la Charte de l'ONU. C'est de cette évolution qu'est issu le Conseil économique et social, prévu à Dumbarton Oaks mais qui n’a pas été inclus parmi les organes principaux quà San Francisco, sous l'influence des petits États. ${ }^{79}$ Les membres de cet organe restreint sont élus par l'Assemblée générale, lorgane plénier auquel il est subordonné. À l'origine, il comprenait 18 États. Pour tenir compte de la rapide progression de la composition de lorganisation, deux amendements à la Charte ont porté ce nombre à 27, en 1965, puis à 54, en 1973. ${ }^{80}$ Depuis la réforme de l'article 61 de la Charte en 1973, il se compose de cinquante quatre membres élus par l’Assemblée générale selon une répartition

DIEZ DE VELASCO VALLEJO (Manuel), Les organisations internationales, op.cit., p.198.

Ibid.

Ibid, p.203.

DREYFUS (Simone), Droit des relations internationales, $4^{\mathrm{e}}$ édition revue et augmentée, Paris, CUJAS, 1992, p.249.

Ibid.

DREYFUS (Simone), Droit des relations internationales, op.cit., p.250. 
géographique rigoureuse, pour une période de trois ans. ${ }^{81}$ La raison essentielle qui fonde létude de cet organe restreint est son rôle dans le système des Nations Unies. Placé au cœur de l'action économique et social de l'ONU, paradoxalement il dépend entièrement de l’Assemblée générale. Cette dépendance pose le problème de son rôle véritable au sein de l'ONU.

En dehors de ces deux organes principaux de l'ONU, sera aussi concerné par notre étude, le Conseil d’administration de l'OIT. Au sujet de l'OIT, il est important de rappeler que la nécessité d'une règlementation internationale du travail apparaît au début du XIXe siècle. Néanmoins, cette préoccupation n'est défendue que par des initiatives privées, et non pas gouvernementales. ${ }^{82}$ À la fin de la première guerre mondiale, la nécessité devient plus aigüe et passe au niveau gouvernemental ; l'idée d'une règlementation internationale étant accueillie à la Conférence de paix de Paris. ${ }^{83}$ À sa première réunion, il est décidé de créer une Commission internationale du travail chargée délaborer un projet d'institution permanente sous les auspices de la SDN. Le projet remanié est adopté par la Conférence de paix du 11 avril 1919 et devient la partie XIII du traité de Versailles. L'OIT commence à fonctionner dès 1919 ; sa première Conférence se réunit à Washington, en 1919. La principale originalité de l'OIT réside dans le fait que des représentants des travailleurs et des employeurs y sont associés à ceux des gouvernements. C'est en cela que consiste la composition tripartite de certains organes de l'OIT. ${ }^{84}$ Parmi ceux-ci, figure le Conseil d'administration. Il comprenait 48 membres (24 représentant les gouvernements, 12 les travailleurs et 12 les employeurs). Un amendement adopté en 1972 a porté ce nombre à 56. Aux termes de l'article 7 de sa Constitution « le Conseil d’administration sera composé de 56 personnes : 28 représentant les gouvernements, 14 représentant les employeurs et 14 représentant les travailleurs.» ${ }^{85}$

Toutefois, l'alinéa 2 de cette disposition précise que « sur les 28 personnes représentant les gouvernements, 10 seront nommés par les membres dont l'importance industrielle est la plus considérable et 18 seront nommées par les membres désignés à cet effet par les délégués gouvernementaux à la Conférence, exclusion faite des délégués des dix membres susmentionnés.» ${ }^{86}$ C'est justement le point touchant à la nomination des 10 représentants par les membres ayant l'importance industrielle la plus considérable que se situe l'intérêt de l'étude du Conseil d'administration de l'OIT. Tel que l'a conçu la Constitution modifiée, le Conseil d'administration constitue une exception au principe de légalité des États. ${ }^{87} \mathrm{Il}$ s'agira de comprendre le fondement de ce privilège attribué à ces États au sein de l'OIT.

\footnotetext{
81 DIEZ DE VELASCO VALLEJO (Manuel), Les organisations internationales, op.cit., p.198.

82 Ibid.

83 Ibid.

84 DREYFUS (Simone), Droit des relations internationales, op.cit., p.286.

85 Voir l'article 7, alinéa 1 de la Constitution de l'OIT.

86 Voir l'article 7, alinéa 2 de la Constitution de l'OIT.

87 DREYFUS (Simone), Droit des relations internationales, op.cit., p.290.
} 
La particularité du Conseil de l'Autorité des fonds marins résulte de sa composition très complexe, qui est le résultat de négociations difficiles entre les grandes puissances et les petits États. ${ }^{88}$ L'intérêt de la communauté internationale pour les ressources minérales existant au fond et dans le sous-sol des mers et des océans, à des grandes profondeurs, est relativement récent. Il s'est renforcé à mesure que se profilait à partir des années cinquante, grâce aux progrès scientifiques et technologiques, la possibilité d'y exploiter non seulement les hydrocarbures mais aussi les nodules polymétalliques qui s'y trouvent, des formations métallifères riches en cobalt, nickel, manganèse et cuivre ${ }^{89}$ Cette possibilité a été accueillie avec une attention et un degré de préoccupations différents selon les pays. Les États industrialisés, par exemple, se sont montrés favorables à un régime libéral d'exploitation des ressources, directement par les États et par les personnes autorisées par eux. ${ }^{90}$ $\mathrm{Au}$ contraire, les pays en développement et en particulier ceux dont les économies dépendaient directement de l'extraction et de l'exportation des minéraux ont rapidement montré les difficultés que supposeraient pour eux l'exploitation industrielle des gisements : d'une part, ils ne disposaient pas, en effet, de la technologie nécessaire, d'autre part, les produits extraits viendraient concurrencer sur les marchés les minéraux exploités sous terre et provoqueraient une chute des prix. ${ }^{91} \mathrm{La}$ définition du régime du fond des mers et des océans est à l'origine de la $3^{\mathrm{e}}$ Conférence des Nations Unies sur le droit de la mer. Aussi, le mandat de la Conférence portait-il sur la réforme du droit de la mer au moyen d'une seule Convention universelle. ${ }^{92}$ La Convention des Nations Unies sur le droit de la mer, appelée encore Convention de Montégo Bay (Jamaïque) est le fruit de cette Conférence. L’ampleur de la tâche entreprise permet de comprendre la longueur d'une négociation multilatérale qui dura 9 ans. Elle a débuté en 1973 et a été ouverte à la signature le 10 décembre $1982 .{ }^{93}$ Mais, elle n’est entrée en vigueur que plus d'une décennie plus tard. ${ }^{94}$ La Convention de 1982 consacre la section 4 de la Partie XI à l'Autorité ; son article 156 crée une Autorité internationale des fonds marins et précise que tous les États parties sont ipso facto membres de l'Autorité dont le siège est à Kingston (Jamaïque) ${ }^{95}$ Larticle 157 de la Convention établit que "l'Autorité est l'organisation par l'intermédiaire de laquelle les États parties organisent et contrôlent les activités menées dans la Zone, notamment aux fins de l'administration des ressources de celle-ci, conformément à la présente partie $" .{ }^{96}$ Parmi les organes de cette organisation internationale, figure le Conseil d'administration. Aux termes de l'article 161 de la Convention de Montégo Bay, le Conseil se compose de 36 membres élus par l'Assemblée pour une période de quatre ans renouvelables (lors de la première élection,

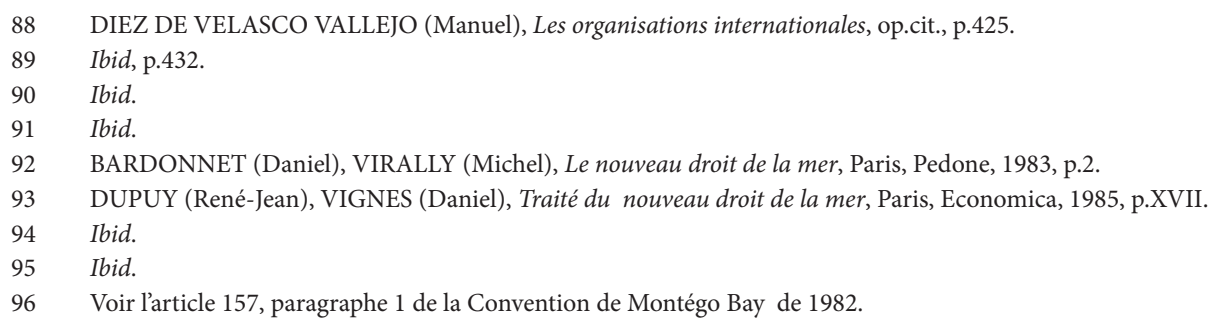


cependant, la durée du mandat de la moitié des membres représentant chacun des groupes d'États est de deux ans).$^{97}$ La durée des négociations qui ont donné naissance à cette convention témoigne de l'intérêt des États pour le contenu du droit destiné à régir l’espace maritime. Une grande partie des débats a porté sur la répartition des pouvoirs entre le Conseil, l’organe restreint, et l'Assemblée, l’organe plénier. La longue énumération des pouvoirs et de fonctions que contient l'article 162 suffit à faire comprendre que le Conseil est le centre du pouvoir de l'Autorité..$^{98} \mathrm{De}$ ce fait, il n'est donc pas étonnant que la composition du Conseil et sa prise de décision aient constitué deux des problèmes les plus épineux de l'histoire de la Conférence et aient été parmi les derniers à être résolus. ${ }^{99}$ Létude de cet organe restreint permettra de comprendre la répartition des pouvoirs entre la Conférence, lorgane plénier et le Conseil, l’organe restreint.

Nous avons aussi retenu le Conseil d’administration du FMI comme objet d'étude. En effet, les origines du FMI remontent à la Conférence monétaire et financière des Nations Unies, réunie à Bretton Woods (Etats-Unis) en juillet 1944. Laccord constitutif du FMI est entré en vigueur le 27 décembre 1945 par la signature de vingt-neuf pays réunissant quatre-vingts pour cent des quotes-parts acceptés à la Conférence de Bretton Woods. Le Conseil d’administration figure parmi les organes principaux prévus par le Statut du Fonds, précisément à la section 3 alinéa (b). ${ }^{100} \mathrm{Il}$ est composé d’administrateurs, chargés de la gestion des opérations du Fonds. Ceux-ci sont au nombre de 20 membres dont 5 sont nommés par les membres qui disposent des quotesparts les plus élevés, et les 15 autres sont élus par les autres États membres. ${ }^{101}$ La composition du Conseil d’administration se fonde sur des considérations essentiellement économiques. Aussi, les problèmes soulevés par celles-ci entre les grandes puissances et les petits États méritent-ils dêtre relevés dans cette thèse.

$\mathrm{Au}$ sein des organisations régionales, le Conseil de paix et de sécurité et la Commission de l'UA ont retenu notre attention. Il est bon de rappeler que la mise en place de l'UA répond à un besoin de modernisation des structures de l'OUA qui, visiblement, nétaient plus adaptées au processus d'intégration du continent dans léconomie mondiale, et dans le règlement des problèmes sociaux, culturels, économiques et politiques. ${ }^{102}$ Une session extraordinaire de la Conférence des Chefs d'États en septembre 1999 à Syrte, en Lybie, avait abouti à une déclaration d'adoption de principe de la création de l'UA. ${ }^{103}$ Un an plus tard à Lomé (Togo), l’Acte constitutif de l’Union était

\footnotetext{
97 Voir l'article 161 de la Convention de Montégo Bay de 1982.

98 DUPUY (René-Jean), VIGNES (Daniel), Traité du nouveau droit de la mer, op.cit., p.635.

99 Ibid.

100 Voir l'article XII, section 3 du Statut du FMI.

101 DIEZ DE VELASCO VALLEJO (Manuel), Les organisations internationales, op.cit., p.396.

102 BAMBA (Kassimi), "La difficile gestation de l'Union Africaine », in Débats, Courrier d'Afrique de l'Ouest, n²627, juillet-août, 2005, p.3.

Ibid.
} 
à son tour adopté, et, en 2001, le sommet de Lusaka (Zambie) validait le programme de mise en place de l'Union. ${ }^{104}$

Parmi les organes de la nouvelle organisation africaine, figurent le Conseil de paix et de sécurité et la Commission. En vue de mettre en place une structure opérationnelle pour la mise en œuvre efficace des décisions prises dans le domaine de la prévention des conflits, du rétablissement de la paix, il a été créé par l'Acte constitutif de l'UA et le Protocole du 9 juillet 2002 signé à Durban (République Sud-Africaine), un Conseil de paix et de sécurité. ${ }^{105}$ C'est le Mali qui a proposé lors du sommet de l'OUA à Lusaka en Zambie en 2001, de transformer le mécanisme de l'OUA pour la prévention, la gestion et le règlement des conflits en un Conseil de médiation et de sécurité avec des membres permanents et non permanents pour dit-on, « tenir compte des responsabilités particulières de certains pays au regard de leurs poids politique, économique ou démographique.» ${ }^{106}$ Le Conseil de paix et de sécurité a des objectifs et une composition similaires à ceux du Conseil de sécurité de l'ONU, mais il se distingue de ce dernier sur plusieurs points. ${ }^{107} \mathrm{Il}$ est composé de 15 membres, mais ne met en place ni de droit de veto, ni de permanence de ses membres. ${ }^{108}$ C’est cette rupture avec le Conseil de sécurité de l'ONU qui explique le choix de cet organe restreint dans le cadre de cette thèse.

La Commission a été officiellement mise en place le 16 septembre 2003. ${ }^{109}$ C'est le Secrétariat de l'Union, à l'image de ce queétait le Secrétariat général de l'OUA, mais avec une ambition d'autonomie plus importante. ${ }^{110}$ Elle est composée d'un Président et d'un Vice-président et de huit Commissaires tous nommés par la Conférence, donc par les Chefs d'État et de gouvernement ou leurs représentants. ${ }^{111}$ Sa particularité découle de sa nature juridique. Elle est un organe intégré. À ce titre, elle est composée de personnalités indépendantes.

Au regard de ce qui précède, notre réflexion se fera en deux grandes parties :

Première partie : Les fondements de l'institution des organes restreints dans les organisations internationales. Nous confronterons les motivations juridiques qui conduisent les États membres à créer les organes restreints avec les réalités politiques qui prévalent dans les organisations internationales.

Deuxième partie : Lordonnancement des pouvoirs des organes restreints. La réflexion sur l'agencement des pouvoirs des organes restreints permettra d'analyser les modalités de répartition

104 Ibid

105 MVELLE (Guy), L'Union Africaine, Fondements, organes, programmes et actions, Paris, L'Harmattan, 2007, p.179.

106 Ibid.

107 DUJARDIN (Stéphanie), «L'Union africaine : objectifs et moyens de gestion des crises politiques et des conflits armés ", in BANGOURA (Dominique) et FIDIECK A BIDIAS (Emile) (dir.), L'Union africaine et les acteurs sociaux dans la gestion des crises et des conflits armés, Paris, L'Harmattan, 2006, p.69.

108 Ibid.

109 MVELLE (Guy), L'Union africaine, fondements, organes programmes et actions, op.cit., p.216.

110 Ibid

111 Ibid. 
des pouvoirs dans les organisations internationales. Ce point étant clarifié, nous vérifierons si les pouvoirs reconnus aux organes restreints sont de nature à assurer l'efficacité des organisations internationales. 


\section{PREMIERE PARTIE}

\section{LES FONDEMENTS DE L'INSTITUTION DES ORGANES RESTREINTS DANS LES ORGANISATIONS INTERNATIONALES}

Parler des fondements de l'institution des organes restreints revient à évoquer les raisons qui justifient leur présence dans les organisations internationales. Elles sont à la fois d’ordre technique et d'ordre politique. ${ }^{112}$

La création d'un organe restreint dans une organisation internationale n'est pas neutre. Elle répond à un besoin fonctionnel ressenti par les États membres. A l'analyse, elle traduit deux finalités contradictoires. S'il est vrai que les organes restreints visent l'efficacité des organisations internationales (Titre I), force est d'admettre qu'ils favorisent l'hégémonie des grandes puissances (Titre II).

112 SUR (Serge), Relations internationales, $6^{\mathrm{e}}$ édition, Paris, Montchrestien, 2011, p.319. 


\section{TITRE I : LA RECHERCHE DE L'EFFICACITE DES ORGANISATIONS INTERNATIONALES}

L’efficacité de toute organisation internationale suppose la mise en œuvre des missions assignées par ses fondateurs que sont les États. ${ }^{113}$ Les organisations doivent pouvoir répondre aux besoins des États membres.

Elles ont une personnalité juridique fonctionnelle. ${ }^{114}$ Contrairement à celle de l'État, la personnalité des organisations internationales est bornée par le principe de spécialité. ${ }^{115}$ Ceci veut dire quelle ne peut exercer les capacités juridiques qui lui sont dévolues que dans la limite et pour la réalisation de l'objet et des buts d'intérêt général que lui assigne sa charte constitutive. ${ }^{116}$ Elles sont limitées aux missions pour lesquelles elles ont été créées. De ce fait, toute l'architecture institutionnelle est résolument tournée vers la mise en œuvre de ses missions. Les organes restreints s'inscrivent dans la recherche d'un meilleur fonctionnement des organisations internationales. De ce point de vue, leur apport se situe tant au plan fonctionnel (Chapitre I) que politique (Chapitre II).

113 YAO-N'DRE (Paul), Droit des organisations internationales, op.cit., p.123 et 124.

114 La personnalité juridique qui a été reconnue aux organisations internationales par la CIJ dans l’avis relatif à l'affaire de «la réparation des dommages subis aux services des Nations unies » du 11 avril 1949 est une personnalité fonctionnelle. Selon la Cour, « l’organisation était destinée à exercer des fonctions et à jouir des droits... qui ne peuvent s'expliquer que si l’organisation possède une large mesure de personnalité internationale. Les droits et obligations reconnus aux organisations internationales visent exclusivement à leur permettre de s'acquitter des missions qui leur ont été assignées par les États membres. Cette personnalité fonctionnelle soppose à celle des États qui jouissent d'une personnalité générale leur permettant d’agir dans tous les domaines de la vie internationale.

115 DUPUY (Pierre-Marie), KERBRAT (Yann), Droit international public, $11^{\mathrm{e}}$ édition, Paris, DALLOZ, 2012, p.202.

116 Ibid. 
CHAPITRE I : LES APPORTS FONCTIONNELS DES ORGANES RESTREINTS DANS LES ORGANISATIONS INTERNATIONALES

Les apports fonctionnels des organes restreints doivent être perçus comme leurs contributions au fonctionnement des organisations internationales. De ce fait, les organes restreints favorisent la célérité dans l'exécution (Section I) et la rationnalisation des missions des organisations internationales (Section II). 


\section{SECTION I : LA CELERITE DANS L'EXECUTION DES MISSIONS DES ORGANISATIONS INTERNATIONALES}

Une organisation internationale doit répondre avec célérité aux problèmes posés par les États membres. Contrairement aux organes pléniers, les organes restreints sont les plus aptes à réagir promptement dans l'exécution des missions de l'organisation internationale. Il est clair que ces organes présentent des avantages en ce qui concerne la facilité de leur réunion et l'efficacité des délibérations. ${ }^{117}$ Cette diligence des organes restreints se perçoit à travers la mise en œuvre de la continuité du service public (Paragraphe I) et leur disponibilité face aux situations d'urgence dans les organisations internationales (Paragraphe II).

\section{PARAGRAPHE I : LA MISE EN CEUVRE DE LA CONTINUITE DU SERVICE PUBLIC DANS LES ORGANISATIONS INTERNATIONALES PAR LES ORGANES RESTREINTS}

Le principe de la continuité du service public est un principe qui régit le fonctionnement de l'administration de l'État. Il signifie qu'elle doit fonctionner de façon ininterrompue et régulière.

Toute organisation internationale revêt, plus ou moins, un caractère de service public, cest-à-dire constitue un ensemble d’organes remplissant des fonctions d'intérêt général. ${ }^{118} \mathrm{La}$ continuité de l'exercice de ses fonctions est un élément essentiel de l'organisation. ${ }^{119}$ Transposé dans lordre interne des organisations internationales, ce principe est une des conditions essentielles de l'efficacité des organisations internationales. ${ }^{120} \mathrm{Il}$ est mis en œuvre dans les organes restreints par lorganisation de réunions périodiques (A) et par la représentation permanente des États membres (B).

117 SUR (Serge), Relations internationales, op.cit., p.319.

118 CHAUMONT (Charles), L'ONU, Que sais-je?, n748, PUF, 1971, p. 25.

119 Ibid.

120 La thèse du service public dans les organisations internationales a été remise en cause par certains auteurs. Parmi ceux-ci, figure le Professeur Riccardo MONACO. A ce sujet, il affirme qu' " en dehors des limitations et des liens qui lui sont imposés par ces règles, l’organisation jouit d'un large pouvoir discrétionnaire. En exerçant ce pouvoir l'organisation doit toutefois observer des critères particuliers, qui sont surtout celui de la bonne gestion administrative et celui de la sauvegarde de l'intérêt public.

Il est difficile de ramener à une conception unitaire les différentes manifestations de l'action concrète qui, en raison de la variété des objectifs, est mise en œuvre par les organisations internationales. Cela explique pourquoi les tentatives qui ont été faites dans ce sens reposent sur une base extrêmement fragile; tel est le cas de la soi- disant théorie générale des services publics internationaux. D’après celle-ci, les différentes tâches qui incombent à chaque organisation internationale devraient être conçues comme des services organisés que lorganisation en cause rend à la communauté internationale.

Mais cela n'est pas toujours vrai, parce que, si l'organisation a des objectifs politiques, sociaux, militaires ou d'information, il devient impossible de ramener son activité à celle consistant à rendre un service public. Voila donc pourquoi les fonctions exécutives des organisations internationales ne peuvent pas être ramenées à la notion unitaire de service public international...» (Voir à ce sujet Riccardo MONACO, « Les principes régissant la structure et le fonctionnement des organisations internationales », op.cit., p.151.) 


\section{A : L'ORGANISATION DE REUNIONS PERIODIQUES DANS LES ORGANES RESTREINTS}

$\mathrm{Au}$ delà des règles de fonctionnement prévues par les chartes constitutives, les organisations internationales doivent pouvoir fonctionner régulièrement afin de répondre aux besoins quotidiens des États membres.

Lorganisation de réunions périodiques dans les organes restreints constitue un atout essentiel dans le fonctionnement régulier des organisations internationales. Elles constituent des éléments d'appréciation de l'efficacité d'une organisation internationale. Un organe restreint qui se réunit régulièrement permet à l'organisation d'examiner les problèmes qui se posent aux États membres. Cette régularité dans le fonctionnement des organisations internationales est rendue possible par la permanence des organes restreints. Ainsi, aux termes de l'article 28, alinéa 1 de la Charte des Nations Unies, il est affirmé que «le Conseil de sécurité est organisé de manière à pouvoir exercer ses fonctions en permanence...» ${ }^{121}$ Que revêt l'idée de permanence du Conseil de sécurité contenue dans cette disposition?

La notion de permanence doit être entendue comme une fonction permettant au Conseil de sécurité de se réunir à tout moment, selon les exigences de la situation. ${ }^{122}$ Selon le Professeur René-Jean DUPUY, «la permanence de l'organisation n’est que la traduction de son indépendance par rapport à ses membres; non permanente, l’organisation reste suspendue à la volonté des États pour chacun de ses actes qu'elle est susceptible d'assurer ; permanente, elle s'affirme face aux États.» ${ }^{123}$

Dans la répartition des compétences entre les différents organes des Nations unies, le Conseil de sécurité se présente comme l’organe d'action de l'organisation. A ce titre, il est nécessaire qu'il puisse réunir ses membres à tout moment. Dans la pratique, le Conseil de sécurité siège effectivement de façon quasi-permanente. Pour preuve, le tableau suivant passe en revue le nombre de réunions organisées par le Conseil de sécurité de 1998 à 2005.

121 Voir l'article 28 de la Charte des Nations Unies. Cette disposition a été directement inspirée par la pratique du Conseil de la SDN. Celui-ci n’ayant pas à la différence du Conseil de sécurité un caractère permanent a fréquemment attiré à ses sessions la présence de Ministres des affaires étrangères, qui ont pu ainsi se concerter utilement sur les grands problèmes internationaux de l'heure.

122 LEPRETTE (Jacques), "Commentaire de l'article 28 de la Charte des Nations Unies ", in COT (Jean-Pierre) et PELLET Alain), Commentaire de la Charte de l'ONU article par article, $2^{\mathrm{e}}$ édition, Paris, Economica, 1991, p.515. DUPUY (René-Jean), Le droit international, Que sais-je?, op.cit.p.92. 


\begin{tabular}{cc}
\hline Années & $\begin{array}{c}\text { Nombre de réunions } \\
\text { du Conseil de sécurité }\end{array}$ \\
\hline 1998 & 121 \\
\hline 1999 & 144 \\
\hline 2000 & 193 \\
\hline 2001 & 212 \\
\hline 2002 & 277 \\
\hline 2003 & 229 \\
\hline 2004 & 230 \\
\hline 2005 & 245 \\
\hline Source $:$ www.un.org & \\
\hline
\end{tabular}

Le Règlement intérieur provisoire du Conseil de sécurité confirme la nécessité du caractère permanent des activités de cet organe restreint. Aux termes de l'article 1 de ce texte, « le Conseil de sécurité, sous réserve des dispositions de larticle 4 relatif aux réunions périodiques, se réunit sur convocation du Président toutes les fois que celui-ci le juge nécessaire et sans que l'intervalle entre les réunions puisse excéder quatorze jours.» ${ }^{124} \mathrm{Il}$ découle de cette disposition que le Président du Conseil à qui incombe la convocation du Conseil doit donc toujours être disponible. ${ }^{125}$ Pour ce faire, il doit prendre toutes les dispositions pour pouvoir être joint à tout instant et s'acquitter de ses responsabilités pratiquement dans l'heure qui suit un évènement pouvant motiver la convocation du Conseil. ${ }^{126}$

Le Conseil de sécurité se transformerait en un organe permanent de consultation, dont lordre du jour pourrait sétendre à tous les grands problèmes du moment qui exigent des décisions rapides, une action à long terme, ou de simples consultations exploratoires. ${ }^{127}$

Cette idée est reprise par le Protocole relatif à la création du Conseil de paix et de sécurité de l'UA. Larticle 8 alinéa 1 de ce texte dispose que "le Conseil de paix et de sécurité est organisé de manière à pouvoir exercer ses fonctions en permanence. $»^{128}$ Cet organe restreint doit pouvoir se réunir assez régulièrement dans son fonctionnement.

L’alinéa 2 de cette disposition confirme cette idée de permanence de cet organe restreint. Il stipule que le Conseil de paix et de sécurité « est convoqué aussi souvent que nécessaire au niveau des représentants permanents, et au moins deux fois par mois. Les ministres et les Chefs d'État et

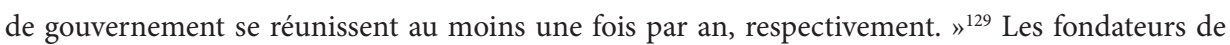
l'organisation africaine ont voulu la doter d'un organe fonctionnant régulièrement. Ses réunions

\footnotetext{
124 Voir l’article 1 du Règlement intérieur provisoire du Conseil de sécurité.

125 LEPRETTE (Jacques), « Commentaire de l’article 28 de la Charte des Nations Unies», op.cit.,p.517.

126 Ibid.

127 VIRALLY (Michel), Lorganisation mondiale, op.cit., p.100.

128 Voir larticle 8 alinéa 1 du Protocole relatif à la création du Conseil de paix et de sécurité de l'UA.

129 Voir l'article 8 alinéa 2 du Protocole relatif à la création du Conseil de paix et de sécurité de l'UA.
} 
périodiques prennent en compte non seulement les Chefs d'État et de gouvernement, mais aussi les représentants permanents des États.

Le même constat peut être établi pour le Conseil d’administration de l'OIT. Les travaux du Conseil sont répartis entre une session complète à l'automne (novembre) et une autre au printemps (mars) ainsi qu'une session d'une journée en juin. ${ }^{130} \mathrm{~A}$ l'exception de la session de juin, les séances plénières du Conseil d'administration ont une durée maximale de trois jours et demi, précédées d'une demi-journée consacrée à des réunions de groupes. ${ }^{131}$ Elles sont précédées de réunions d'une semaine et demi de Commissions et Comités lors des sessions ordinaires et de deux semaines et demi lors de la session précédant les années budgétaires. ${ }^{132}$ Le Conseil d’administration est la plaque tournante d'où émanent toutes les activités de l'organisation et vers laquelle elles convergent au cours et à la fin de leur exécution. ${ }^{133}$ Il établit lordre du jour de la Conférence et est chargé par celle-ci de donner suite aux résolutions qu’elle adopte. ${ }^{134}$

Larticle XII, section III alinéa (g) du Statut de l'OIT dispose que " le Conseil d'administration siège en permanence au siège du Fonds et se réunit aussi fréquemment que l'exige la conduite des affaires du Fonds.» ${ }^{135}$ L'idée de permanence est bien précisée dans ce texte.

Lorganisation de réunions périodiques favorise considérablement le fonctionnement régulier des organisations internationales. Pour ce faire, ces réunions périodiques exigent aussi la représentation permanente des États membres dans les organes restreints.

\section{B : LA REPRESENTATION PERMANENTE DES ÉTATS MEMBRES DANS LES ORGANES RESTREINTS}

La représentation permanente des États membres dans les organes restreints implique la présence de façon ininterrompue de leurs représentants au sein des ces organes. Les États sont représentés dans les divers organes par des personnes qui sont déléguées par leurs gouvernements. ${ }^{136}$ Cette représentation est continue, en ce sens que les États membres sont tous représentés dans ces organes chaque fois qu'ils fonctionnent. ${ }^{137}$

Larticle 13 du Règlement intérieur provisoire du Conseil de sécurité définit les conditions de la représentation permanente des États dans cet organe. Cette disposition prévoit que « chaque membre du Conseil de sécurité est représenté aux réunions du Conseil de sécurité par un

130 OIT, Recueil des règles applicables au Conseil d'administration de l'OIT, Genève, février 2006, p.8.

131 Ibid.

132 Ibid.

133 MONTCEAU (Marc), Lorganisation internationale du travail, Que sais-je ? n836, PUF, 1972, p.29.

134 Ibid.

135 Voir l'article XII, section III, alinéa (g).

136 CHAUMONT (Charles), L'ONU, Que sais-je? nº748, op.cit., p.22.

Ibid. 
représentant accrédité. Les pouvoirs des représentants au Conseil de sécurité sont communiqués au Secrétaire général vingt-quatre heures au moins avant que ces représentants occupent leur siège au Conseil de sécurité. Les pouvoirs doivent émaner soit du Chef de l'État ou du Chef du gouvernement, soit du Ministre des affaires étrangères. Le Chef du gouvernement ou le Ministre des affaires étrangères de chaque membre du Conseil de sécurité est autorisé à siéger au Conseil de sécurité sans présenter de pouvoirs. $»^{138}$

Cette disposition appelle un commentaire. Il sagit de lobligation de chaque membre du Conseil de sécurité de se faire représenter par un représentant accrédité. Cette représentation permanente est la condition essentielle du fonctionnement régulier et permanent du Conseil de sécurité. Ce sont ces représentants permanents accrédités qui permettent d’assurer le fonctionnement ininterrompu du Conseil de sécurité. ${ }^{139}$ L'Ambassadeur Jacques LEPRETTE (ancien Ambassadeur de la France à l'ONU) précise que «les États membres ont établi à New York des représentations permanentes auprès des Nations Unies; ces missions ont un statut diplomatique; elles sont en mesure de suivre quotidiennement les travaux de lorganisation; elles peuvent représenter leur gouvernement à toutes réunions auxquelles celui-ci est convié. Ces observations valent au premier chef pour le Conseil de sécurité.» ${ }^{140}$

La représentation permanente des États au Conseil de sécurité est réalisée aussi par le statut de membres permanents dont jouissent certains États. Le statut de membres permanents, en marge des avantages qu'il procure à ses bénéficiaires au plan politique, s’accompagne de nombreuses charges administratives. Monsieur Jacques LEPRETTE affirme encore que " pour beaucoup, ce statut évoque principalement un privilège partagé par quatre autres États : celui de faire obstacle à toute résolution du Conseil portant sur le fond d'une affaire qui lui a été soumise.

138 Voir l’article 13 du Règlement intérieur provisoire du Conseil de sécurité.

139 La représentation permanente se réalise à travers les missions permanentes dont dispose chaque État auprès des Nations Unies. Le Professeur VIRALLY soutient que « chacun des membres du Conseil de sécurité doit entretenir au siège une mission diplomatique permanente composée de son représentant au Conseil et de ses collaborateurs. Aucune obligation de cette nature n'existait pour les autres États membres, puisque leur participation aux travaux de l'organisation était limitée aux sessions de l’Assemblée générale (et des autres Conseils dont ils pouvaient être membres).

Très rapidement, cependant, nombre d'États comprirent l'avantage de disposer d'une mission permanente, grâce à laquelle ils pourraient être tenus constamment informés de lévolution des affaires des Nations Unies et maintiendraient un contact régulier avec le Secrétariat [...] Il était aussi dans l'intérêt de l'organisation ellemême que de telles missions fussent établies, car cela permettait à ses organes (et spécialement au Conseil de sécurité et au Secrétariat) de disposer d'un canal pour entrer en contact avec les gouvernements des États membres chaque fois que cela pouvait être désirable [...]

Depuis lors, le rôle et les effectifs des missions permanentes n’ont cessé de croître. Par les rapports qu'elles entretiennent entre elles, sur une base permanente, elles facilitent considérablement la préparation des sessions de l’Assemblée générale [...]

Les missions permanentes fournissent en outre un réservoir de délégués susceptibles de prendre part aux travaux des multiples organes, principaux ou subsidiaires, qui se réunissent désormais dans l'intervalle des sessions de l'Assemblée générale, de façon presqu' ininterrompue. " (Voir VIRALLY Michel, Lorganisation mondiale, op.cit., p. 114-115).

140 LEPRETTE (Jacques), «Commentaire de l'article 28 de la Charte de l’ONU », op.cit., p.516. 
Cela n'est pas faux... On a tendance à faire passer au second plan l'essentiel : la permanence. Cinq membres de lorganisation ont participé, sans discontinuité, et participent à toutes les sessions du Conseil.» ${ }^{141}$ Le statut de membres permanents du Conseil de sécurité ne comporte pas que des prérogatives exorbitantes au plan politique. Bien au contraire, selon l'Ambassadeur LEPRETTE « ce statut implique une organisation appropriée des missions qui ont à y faire face. La charge de travail est considérable. ${ }^{142}$ Les cinq se sont équipés en conséquence, car le statut de membre permanent exige une disponibilité de tous les instants.» ${ }^{143}$

La représentation des États dans l'organisation se fait par le moyen de personnes qui, considérées dans leur ensemble, constituent "une délégation ». ${ }^{144}$ Au Conseil de sécurité des Nations Unies, les missions permanentes qui sont installées à New York pour représenter les États auprès de l’organisation sont de véritables ambassades, composées généralement de diplomates professionnels. $^{145}$

Construit sur le modèle du Conseil de sécurité des Nations Unies, le Conseil de paix et de sécurité de l'UA incarne aussi l'idée de continuité du service public dans cette organisation internationale régionale. Elle transparaît clairement dans les dispositions du Protocole relatif à la création du Conseil de paix et de sécurité de l'UA. Aux termes de l'article 8 alinéa 1 du Protocole, ${ }^{146}$ il ressort que «... chaque membre du Conseil de paix et de sécurité doit avoir, en tout temps, un représentant au siège de l'Union.»

Lorganisation de réunions périodiques et la représentation permanente des États au sein des organes restreints concourent considérablement à la mise en œuvre de la continuité du service public dans les organisations internationales. ${ }^{147}$ Toutefois, cette contribution ne se limite pas à ce niveau. Les organes restreints se caractérisent aussi par leur disponibilité face aux situations d'urgence dans les organisations internationales.

141 LEPRETTE (Jacques), « La France au Conseil de sécurité », in La France et l'ONU, depuis 1945, Panoramiques-Corlet, Paris, 1995 p.167.

142 Selon l'Ambassadeur LEPRETTE, « la mission des États-Unis compte dans ses rangs des experts qui ont presque vingt ans d'ancienneté au Conseil de sécurité. L'URSS avait mis au point une formule qui faisait passer ses spécialistes des Nations Unies par New York, Moscou et le Secrétariat de l'organisation. Ces agents se distinguaient par une maîtrise incomparable des dossiers.» (Voir « la France au Conseil de sécurité », déjà cité, p.167) Ibid.

144 CHAUMONT (Charles), L'ONU, Que sais-je ? nº748, op.cit., p.26.

145 Ibid.

146 Voir l’article 8 alinéa $1 \mathrm{~d}$ u Protocole relatif à la création du Conseil de paix et de sécurité de l'UA.

147 Lorganisation de réunions périodiques et la représentation permanente des États au sein des organes restreints permettent aussi de distinguer les organisations internationales des Conférences diplomatiques. La Conférence diplomatique est une institution dépourvue de permanence et qui réunit à certaines périodes, fixes ou à fixer, les États membres aux fins d'examen des problèmes d'intérêt commun. La Conférence diplomatique apparaît comme un cadre dont le contenu varie selon la conjoncture économique et politique. Le trait distinctif de la Conférence diplomatique par opposition à l'organisation internationale, réside dans l'absence de permanence de ses organes. 


\section{PARAGRAPHE II : LA DISPONIBILITE DES ORGANES RESTREINTS FACE AUX SITUATIONS D’URGENCE DANS LES ORGANISATIONS INTERNATIONALES.}

Les problèmes auxquelles les organisations internationales sont appelées à faire face peuvent avoir un caractère urgent. C'est surtout dans les situations de crises politiques que l'urgence se ressent le plus dans les relations internationales. Dans ces cas, les organes restreints se révèlent être les plus aptes à réagir efficacement.

La disponibilité des organes restreints se manifeste par la mise en place d'un système de réaction rapide fondé sur ceux-ci dans les organisations internationales (A). Mais, ce système qui devrait permettre aux organisations internationales d'agir promptement comporte de nombreuses lacunes (B).

\section{A : LA MISE EN PLACE D’UN SYSTEME DE REACTION RAPIDE FONDE SUR LES ORGANES RESTREINTS}

Les organisations internationales sont apparues dans les relations internationales dans le but de permettre aux États membres de résoudre les difficultés qui se présentent à eux. Or, parmi celles-ci, certaines revêtent un caractère éminemment urgent. ${ }^{148}$ Dans ces conditions, la question se pose de savoir quel est l'organe des organisations internationales qui peut réagir rapidement dans une telle situation?

Au regard du nombre d'États qui les composent, les organes pléniers seraient inaptes à réagir rapidement et efficacement. Il faudrait un temps relativement long pour réunir une centaine d'États et prendre une décision dans le but de résoudre une crise imminente et actuelle. Les organes pléniers n’ont pas la possibilité de programmer leurs réunions de façon régulière dans l’année. Les réunions des ces organes sont périodiques : le rythme des sessions ordinaires est d'une périodicité allant de un à cinq ans. ${ }^{149}$ Les statuts des organisations prévoient en général la convocation

\footnotetext{
148 Dans les organisations internationales à caractère politique comme l'ONU et l'UA, les problèmes relatifs à la paix et la sécurité peuvent avoir un caractère éminemment urgent. Tel fut le cas de la crise post-électorale ivoirienne de novembre 2010 à avril 2011. En effet, face aux nombreuses violations des droits de l'homme résultant des affrontements armés entre les partisans de M. Laurent GBAGBO, président sortant, et ceux du candidat Alassane OUATTARA, l'UA et l'ONU se sont saisies de l'affaire pour trouver une solution rapide à cette crise politique. Aussi, l'intervention de ces deux organisations internationales s'est-elle faite à travers leurs organes restreints respectifs que sont le Conseil de paix et de sécurité (UA) et le Conseil de sécurité (ONU) (Voir la deuxième partie de notre thèse). NGUYEN QUOC (Dinh), Droit international public, op.cit.,p.686.
} 
périodique (annuelle pour l'ONU, ${ }^{150}$ biannuel pour la FAO et l'UNESCO) de l'Assemblée plénière pour des sessions ordinaires. Souvent, des sessions extraordinaires peuvent être tenues sur demande d'une majorité des membres. ${ }^{151}$

Logiquement les organes qui sont aptes à réagir promptement aux situations de crises dans les organisations internationales sont les organes restreints. Les raisons de cette efficacité des organes restreints s'explique essentiellement par le nombre réduit des États membres. En effet, ces organes peuvent se réunir plus facilement. ${ }^{152}$ Le nombre limité de membres est de nature à favoriser une concertation spontanée dans une situation de crise. Dans cette optique, les membres fondateurs des Nations Unies ont confié le dispositif de sécurité collective qui est la mission fondamentale de cette organisation internationale au Conseil de sécurité. Larticle 24, paragraphes 1et 2 de la Charte de l'ONU, consacre assez clairement cette intention des membres fondateurs des Nations Unies. Selon cette disposition « afin d'assurer l'action rapide et efficace de l'organisation, ses membres confèrent au Conseil de Sécurité la responsabilité principale du maintien de la paix et de la sécurité internationale et reconnaissent qu'en s'acquittant des devoirs que lui imposent cette responsabilité le Conseil de sécurité agit en leur nom ... ${ }^{153}$ Cette disposition traduit l'image que les fondateurs de l'ONU ont du Conseil de sécurité. Le Professeur VIRALLY souligne que « le Conseil ne connaît que des différends et des situations dont il est saisi, et n'est saisi que lorsque la paix est compromise ou plus ou moins directement menacée. Son intervention est donc marquée par l'urgence et la crise et se développe très souvent dans une atmosphère dramatique. Elle n'est qu'une réaction à des évènements qui se sont produits en dehors de lui et doit, au moins dans un premier temps, aboutir à des mesures ayant un effet immédiat. ${ }^{154}$ Les Etats membres ont délégué leurs compétences au Conseil de sécurité parce qu'il peut réagir promptement.

Mais, au-delà de cette simple délégation de compétence ${ }^{155}$ des membres des Nations Unies au Conseil de sécurité, une question mérite d’être posée. Pourquoi le choix des membres fondateurs

150 L’Assemblée générale de l'ONU tient une session ordinaire annuelle dont l’ouverture est fixée au troisième mardi de septembre. En cas de besoin, des sessions extraordinaires peuvent être convoquées sur la demande soit du Conseil de sécurité, soit de la majorité des États membres, soit d'un seul membre à condition que cette demande soit appuyée par la majorité. L’Assemblée elle-même peut, bien entendu, décider elle-même de se réunir en session extraordinaire. Lorsqu'elle fait appel à la procédure des sessions extraordinaires d'urgence, prévue par la résolution 377 (V) 1950, l’Assemblée doit se réunir dans les vingt-quatre heures suivant sa convocation. Réunie en session extraordinaire, l'Assemblée ne peut s'occuper que de la question ou des questions pour lesquelles elle a été convoquée, sauf décision contraire prise à la majorité des deux tiers. En revanche, ses sessions ordinaires sont destinées à permettre aux représentants des États membres, généralement les Chefs État, de se prononcer sur les grands problèmes qui se posent dans les relations internationales. SEIDL-HOHENVELDERN (Ignaz), «Les organes des organisations internationales », in Manuel sur les organisations internationales, Martinus Nijhoff publihers, 1988, p.84 et 85. Ibid, p.93.

153 Voir l'article 24 alinéa 1 de la Charte de l'ONU. VIRALLY (Michel), Lorganisation mondiale, op.cit., p.100. ticle par article, $3^{\mathrm{e}}$ édition, Paris, Economica, 2005, p.883. 
des Nations Unies s'est porté sur un organe restreint qui est congénitalement limité par le droit de veto ? $^{156}$ La réponse à cette interrogation nous est fournie par le Professeur DEGNI-SEGUI en ces termes : «le choix du Conseil de sécurité s'explique par le nombre et la qualité de ses membres [...] Le Conseil de sécurité offre, en effet, par rapport à l'Assemblée générale, l'avantage dêtre un organe restreint (15 membres) au sein duquel les cinq grandes puissances ont un siège permanent et disposent en outre du droit de veto.» ${ }^{157}$ Cette position du Professeur DEGNI-SEGUI est partagée par le Professeur Charles CHAUMONT. Pour lui « le Conseil de sécurité, organe restreint est compétent pour l'examen des cas concrets, soit au niveau du règlement des litiges (chapitre VI), soit au niveau de la localisation des conflits (chapitre VII).Pour cette compétence, il faut la concentration de la puissance (avec les membres permanents) dans une suffisante représentation des continents (avec les membres non permanents).» ${ }^{158}$

Dans le système de sécurité collective des Nations Unies, toutes les conditions juridiques ont été mises en œuvre afin que le Conseil de sécurité puisse effectivement réagir rapidement dans les situations d'extrême urgence. Aux termes de l'article 40 de la Charte, « en cas de menace contre la paix, de rupture de la paix ou d'acte d'agression, avant d'user des pouvoirs qu'il détient au titre des autres dispositions du chapitre VII, le Conseil de sécurité peut édicter des mesures provisoires destinées à prévenir toute aggravation de la situation, sans préjuger des droits et prétentions des parties concernées ni des actions à entreprendre en vue de maintenir ou de rétablir une paix durable.» ${ }^{159}$

La notion de mesures provisoires est significative. Elle n'est pas sans rappeler dans son inspiration les diverses techniques de sursis à exécution, de procédure d'urgence, de décisions avant-dire droit ou d'indication de mesures conservatoires, ${ }^{160}$ dont la mise en ouvre vise à opérer une stabilisation immédiate des situations susceptibles de dégénérer en tension grave ou en conflit ouvert. ${ }^{161}$ Lobjectif visé par de telles mesures est de trouver des solutions immédiates opportunes à la situation de crise qui prévaut. Le Professeur Jared SCHOTT parle même d'un « emergency power of the Security council ». ${ }^{162}$ Il évoque le pouvoir d'urgence du Conseil de sécurité de l'ONU. De ce fait, il soutient que « the architecture of emergency power offers valuable descriptive and normative insight into the legal space in which the security council operates $»{ }^{163}$ Le pouvoir d'urgence du

\footnotetext{
156 La question du droit de veto sera abordée dans le deuxième titre de cette première partie.

157 DEGNI-SEGUI, «Commentaire de l'article 24 paragraphe 1et 2 de la Charte de l'ONU », op.cit.p.883.

158 CHAUMONT (Charles), «Léquilibre des organes politiques des Nations Unies et la crise de l'organisation», AFDI, 1965, p.433.

159 SIMON (Denys), "Commentaire de l'article 40 de la Charte des Nations Unies », in COT (Jean-Pierre), PELLET (Alain) (dir.), La Charte des Nations Unies, commentaire article par article, $2^{\mathrm{e}}$ édition, Paris, Economica, 2005, p.667.

$160 \quad$ Ibid, p.668

161 Ibid.

162 SCHOTT (Jared), " Chapter VII as exception : Security council action and the regulative ideal of emergency ", Northen Journal of International Human Rights, Volume 6, Issue 1, 2007, p.26-27.

Ibid.
} 
Conseil de sécurité lui permet dans le cadre de la Charte d’agir promptement face à une crise qui surgit dans lordre international.

La procédure d'urgence de l'UA s'inscrit dans la même logique que celle de l'ONU. Elle fonctionne autour de deux organes restreints que sont le Conseil de paix et de sécurité et la Commission. Larticle 1 paragraphe 1 du Protocole relatif à la création du Conseil de paix et de sécurité dispose que "...Le Conseil de paix et de sécurité constitue un système de sécurité collective et d'alerte rapide, visant à permettre une réaction rapide et efficace aux situations de conflits en Afrique.» ${ }^{164}$ A l'instar du Conseil de sécurité des Nations Unies, le Conseil de paix et de sécurité de l'UA est un organe restreint autour duquel se centralise le mécanisme de "prévention, de gestion et de règlement des conflits.» ${ }^{165}$ Toutefois, pour exécuter ses missions, le Conseil de paix et de sécurité « est appuyé par la Commission, un groupe de sages, ainsi que par un système continental d'alerte rapide, une force africaine pré positionnée et un Fonds spécial.» ${ }^{166}$

Sur le plan politique, la réaction rapide de l'UA face aux crises qui secouent le continent contraste avec la pratique de l'OUA fondée sur le principe de non-ingérence dans les questions internes des États membres qui a fini par cautionner tous les coups d'État jusqu’en 1998, légaliser puis légitimer les « alternances » au moyen des insurrections armées. ${ }^{167} \mathrm{~A}$ la différence de l'OUA, la Charte de l'UA en son article 4 paragraphe (h) prévoit un « droit de l'Union d'intervenir dans un État membre [...] dans certaines circonstances graves, à savoir : les crimes de guerre, le génocide et les crimes contre l'humanité,» ${ }^{168}$ C'est cette disposition qui permet au Conseil de paix et de sécurité de réagir rapidement aux crises qui éclatent dans les États membres. ${ }^{169}$

Le système de réaction rapide des organisations internationales mis en place à travers les organes restreints permettent à ceux-ci de s'acquitter efficacement de leurs missions. Mais, il n’a pas toujours fonctionné normalement pour des raisons diverses.

\section{B : LES LIMITES DU SYSTEME DE REACTION RAPIDE FONDE SUR LES ORGANES RESTREINTS}

Evoquer les limites du système de réaction rapide fondé sur les organes restreints revient à montrer les causes et les manifestations de son mauvais fonctionnement.

La matérialisation du système de sécurité collective des Nations Unies devrait se réaliser par la mise en place d'un Comité d'état-major, responsable de la direction stratégique de toutes les

164 Voir l'article 2, paragraphe 1 du Protocole relatif à la création du Conseil de paix et de sécurité de l'UA.

165 Voir l'article 2, paragraphe 1 du protocole relatif à la création du Conseil de paix et de sécurité de l'UA.

166 Voir l'article 2, paragraphe 2 du Protocole relatif à la création du Conseil de paix et de sécurité de l'UA.

167 YAO (Kouassi), "L'Union Africaine : OUA bis ou essai transformé ? ", in Débats, courrier d'Afrique de l'Ouest, op.cit., p.19.

168 Ibid.

169 Dans la deuxième partie de notre réflexion, nous analyserons de façon plus détaillées quelques interventions du Conseil de paix et de sécurité dans les États membres. 
forces armées mises à la disposition du Conseil de sécurité. La constitution de ce Comité détatmajor est prévue par l'article 47 alinéa 1 de la Charte en ces termes : " Il est établi un Comité d’état-major chargé de conseiller et d'assister la Conseil de sécurité pour tout ce qui concerne les moyens d’ordre militaire nécessaires au Conseil pour maintenir la paix et la sécurité internationales, l'emploi et le commandement des forces mises à sa disposition, la règlementation des armements et le désarmement éventuel... ${ }^{170}$ L'intention des rédacteurs de la Charte était évidemment de doter le Conseil de sécurité d'une force militaire afin de rendre ses actions en matière de prévention et de recherche de la paix plus efficaces. Devant contribuer à prendre des décisions rapides, le Comité détat-major devrait forcément avoir une composition restreinte et permanente permettant l'unité de vue et la stabilité. ${ }^{171}$ Aux termes de l’alinéa 2 de l’article 47, « le Comité d’état-major se compose des chefs détat-major des membres permanents du Conseil de sécurité ou de leurs représentants. Il convie tout membre des Nations Unies qui n’est pas représenté au Comité d’une façon permanente à s’associer à lui, lorsque, la participation de ce membre à ses travaux lui est nécessaire pour la bonne exécution de sa tâche. $»^{172}$

Dans les faits, la constitution de ce Comité deétat-major n’a jamais vu le jour. ${ }^{173}$ En effet, de février 1947 à août 1948, un Comité, sur mandat du Conseil de sécurité, étudie en vain sa mise en place, mais échoue contre les divergences entre les occidentaux et l'URSS. ${ }^{174} \mathrm{Il}$ en résulte une absence d'une armée onusienne ${ }^{175}$, apte à intervenir à tout moment et en tout lieu au nom du Conseil de sécurité. De ce fait, l'action du Conseil de sécurité en cas de menace à la paix, de rupture de la paix et d’agression est considérablement handicapée par le défaut d’une force militaire. Celleci demeure selon l’expression de Philippe MOREAU DEFARGES « une virtualité à la concrétisation incertaine.» ${ }^{176}$

170 Voir l'article 47 de la Charte des Nations Unies.

171 MARTINEZ (Jean-Claude), "Commentaire de l'article 47 de la Charte des Nations Unies », in COT (JeanPierre), PELLET (Alain) (dir.), La Charte des Nations Unies, commentaire article par article, $2^{\mathrm{e}}$ édition, Paris, Economica, 2005, p.748.

172 Voir l'article 47, alinéa 2 de la Charte des Nations Unies.

173 CORTEN (Olivier), "La sécurité collective, un rêve contrarié », in Le monde diplomatique, Septembre 2005, p.17.

174 MOREAU DEFARGES (Philippe), Lordre mondial, Armand Colin, Paris, p.71. Lauteur souligne également « qu’en juin 1948, le Secrétaire général, le norvégien Trygve Lie, proposa la création d'une garde des Nations Unies destinée à aider les missions sur place de l’ONU. Le Conseil de sécurité écarte cette proposition. Périodiquement des suggestions sont avancées : en 1971, proposition du Président Nixon, d’une « Réserve des Nations Unies ", formée de contingents nationaux entraînés au maintien de la paix ; en 1982, rapport Palme (groupe permanent de médiateurs, mise en attente de contingents nationaux, revitalisation du Comité détat major). En 1992, à la demande du Conseil de sécurité, le Secrétaire général, légyptien BOUTROS-GHALI, élabore un rapport sur le maintien de la paix, l'Agenda pour la paix, notamment préconisant des « unités d'imposition de la paix » formées de militaires volontaires, capables d'intervenir immédiatement sur mandat du Conseil de sécurité et sous commandement du Secrétaire général ».

Ibid.

176 Ibid, p.72. 
Le système de sécurité collective établi par la Charte était resté incomplet. La mise sur pied de l'instrument militaire qui en constituait la pièce maîtresse avait été renvoyée à plus tard et nécessitait des négociations très concrètes, à mener avec chacun des membres de lorganisation et, en premier lieu, avec les cinq membres permanents. ${ }^{177}$ L'idée d'une action rapide et efficace du Conseil de sécurité est difficile à mettre en pratique à défaut d'une force militaire à sa disposition. L'instrument d'action que constitue le Conseil de sécurité pour l’ensemble des membres siégeant à l'Assemblée générale est dépourvu d’une force militaire permanente.

Mais, son absence a été suppléée par des mécanismes informels qui révèlent la volonté des grands États de garder le contrôle de leurs moyens militaires. ${ }^{178}$ A partir de 1950, et de l'intervention en Corée, on a assisté à la constitution de forces des Nations Unies désignées comme des « casques bleus » composés au cas par cas, de soldats qui sont juridiquement sous l'autorité de l'ONU mais qui proviennent des contingents nationaux. ${ }^{179}$ L'ONU dépend donc de la bonne volonté de ses États membres. ${ }^{180}$

L'une des raisons qui expliquent l'institution des organes restreints semble être la nécessité d'une réaction rapide des organisations internationales face aux situations d'urgence. Mais à celleci, il convient d'adjoindre la rationalisation fonctionnelle des organisations internationales.

\section{SECTION II : LA RATIONNALISATION FONCTIONNELLE DES ORGANISATIONS INTERNATIONALES}

Il faut entendre par la rationalisation fonctionnelle des organisations internationales, une répartition des différentes missions de celles-ci entre ses divers organes. L'institution des organes restreints participe considérablement à cet objectif de rationalisation des organisations internationales. Cette contribution des organes restreints se réalise surtout par la déconcentration des activités des organisations internationales (Paragraphe I). Toutefois, elle est dévoyée par les États membres des organisations internationales (Paragraphe II).

\section{PARAGRAPHE I : LA DECONCENTRATION DES ACTIVITES DES ORGANISATIONS PAR LE TRUCHEMENT DES ORGANES RESTREINTS}

La déconcentration est une technique juridique du droit interne étatique. Elle s'inscrit dans la logique de l'organisation administrative de l'État. Concrètement, elle consiste à conférer des pouvoirs de décision à des organes qui agissent au nom et pour le compte de l'État. Cependant, elle peut aisément être transposée dans lordre interne des organisations internationales. Ainsi, cette

177 VIRALLY (Michel), Lorganisation mondiale, op.cit., p.469.

178 CORTEN (Olivier), "La sécurité collective, un rêve contrarié », op.cit., p.17.

179 Ibid.

180 Ibid. 
transposition de la déconcentration dans les organisations internationales peut s'accomplir par le canal des organes restreints de celles-ci. Au sein de ceux-ci, la pluralité des missions (A) semble justifiée la nécessité de la déconcentration (B).

\section{A : LA PLURALITE DES MISSIONS DES ORGANISATIONS INTERNATIONALES}

La création de toute organisation internationale n'est pas neutre. Selon le Professeur René-Jean DUPUY « l'organisation internationale [...] évoque beaucoup plus la notion de service public $»{ }^{181} \mathrm{Il}$ en résulte qu'elles reçoivent des missions multiples des États. Mais en vertu du principe de spécialité, leurs missions sont définies par les chartes constitutives.

Ces missions des organisations internationales recouvrent toutes les sphères d'activités de la vie internationale. Celles qui illustrent bien cette réalité sont les organisations internationales à vocation générale. Elles reçoivent des missions diversifiées dans leurs Chartes constitutives. L'ONU dans sa Charte constitutive a défini les buts qu'elle s'assigne. Le Chapitre I, intitulé «buts et principes ", comporte deux articles : le premier est consacré aux buts et le deuxième énumère les « principes ». ${ }^{182}$ Les buts qui nous intéressent particulièrement ici constituent la raison dêtre de l'organisation, les fins communes de l'organisation ${ }^{183}$.

Le contenu des buts des Nations Unies est précisé à l’article 1 de la Charte. Aux termes de cette disposition, les buts des Nations Unies sont : «1- Maintenir la paix et la sécurité internationales et à cette fin prendre des mesures collectives efficaces en vue de prévenir et d'écarter les menaces à la paix et de réprimer tout acte d'agression ou autre rupture de la paix, et de réaliser, par des moyens pacifiques, conformément aux principes de la justice et du droit international, l'ajustement ou le règlement de différends ou de situations, de caractère international, susceptible de mener à une rupture de la paix ;

2- Développer entre les nations des relations amicales fondées sur le respect du principe d'égalité des droits des peuples et de leur droit à disposer d'eux-mêmes, et de prendre toutes mesures propres à consolider la paix dans le monde ;

3- Réaliser la coopération internationale en résolvant les problèmes internationaux dordre économique, social, intellectuel ou humanitaire, en développant et en encourageant le respect des droits de l'homme et des libertés fondamentales pour tous, sans distinction de race, de sexe, de langue ou de religion;

4- être un centre où s'harmonisent les efforts des Nations vers ces fins communes. »

\footnotetext{
181 DUPUY (René-Jean), «État et organisation internationales », op.cit., p.14.

182 DIEZ DE VELASCO VALLEJO (Manuel), Les organisations internationales, op.cit., p.139.

183 Ibid.
} 
Le premier constat qui frappe à l'esprit est la pluralité des missions assignées aux Nations Unies. Celles-ci investissent presque tous les domaines de la vie internationale. De façon synthétique, elles se ramènent au tryptique de la paix, de la liberté et du développement. ${ }^{184}$

Dans son Acte constitutif, l'UA s'est orientée dans la même logique que l'ONU. Ces buts mentionnés à l'article 3 de l’Acte constitutif se résument essentiellement à :

- la réalisation de l’unité et de la solidarité entre les pays africains et entre les peuples d'Afrique ;

- la défense de la souveraineté, l’intégrité territoriale et l’indépendance de ses États membres;

- accélération de l'intégration politique et socio économique du continent ;

- la promotion de la paix, la sécurité et la stabilité sur le continent ;

- la promotion des principes et des institutions démocratiques, la participation populaire et la bonne gouvernance....etc.

En dehors des organisations à vocation générale, il y a les organisations spécialisées qui recouvrent aussi des buts très larges. Ainsi, le Fonds Monétaire International et l'Autorité des Fonds Marins peuvent servir amplement d'illustration ici.

Intéressons-nous au FMI. Ses buts sont vastes, et ils recouvrent essentiellement, mais pas exclusivement, deux grands objectifs, qui consistent à favoriser les échanges du commerce international sur une base monétaire multilatérale et d’aider les États membres à équilibrer leur balance des paiements. ${ }^{185}$ Ainsi, aux termes de l'article 1 des Statuts du Fonds, les objectifs sont les suivants :

a) Promouvoir la coopération monétaire internationale au moyen d'une institution permanente, fournissant un mécanisme de consultation et de collaboration en ce qui concerne les problèmes internationaux;

b) Faciliter l'expansion et l'accroissement harmonieux du commerce international et contribuer ainsi à l'instauration et au maintien de niveaux élevés d’emploi et de revenu réel et au développement des ressources productives de tous les États membres, objectifs premiers de la politique économique ;

c) Promouvoir la stabilité des changes, maintenir entre les États membres des régimes de change ordonnés et éviter les dépréciations concurrentielles des changes;

d) Aider à établir un système multilatéral de règlement des transactions courantes entre les États membres et à éliminer les restrictions de change qui entravent le développement du commerce mondial ;

184 LACHS (Manfred), " Commentaire de l'article 1 de la Charte de l'ONU », in COT (Jean-Pierre) et PELLET (Alain) (dir.), La Charte des Nations Unies, commentaire article par article, $2^{\mathrm{e}}$ édition, Paris, Economica, 1991, p.24. DIEZ DE VELASCO VALLEJO (Manuel), Les organisations internationales, op.cit., p.394. 
e) Donner confiance aux États membres en mettant les ressources générales du Fonds temporairement à leur disposition moyennant des garanties adéquates, leur fournissant la possibilité de corriger les déséquilibres de leurs balances de paiements sans recourir à des mesures préjudiciables à la prospérité nationales ou internationale ;

f) Conformément à ce qui précède, abréger la durée et réduire l’ampleur des déséquilibres des balances des paiements des États membres.

En dépit de sa nature d’organisation internationale spécialisée, le FMI bénéficie d’un domaine d'intervention très large.

Les organisations internationales contemporaines bénéficient d'un domaine d'activités très important. Il est donc nécessaire que les États membres procèdent à une déconcentration de leurs activités.

\section{B : LA NECESSITE DE LA DECONCENTRATION DES ORGANISATIONS INTERNATIONALES}

Les nombreuses missions dévolues aux organisations internationales ne peuvent être exécutées seulement par les organes pléniers.

Conscients de cette situation, les États membres des organisations internationales vont instituer des organes restreints en vue de repartir les différentes missions des organisations internationales. La déconcentration des organisations internationales s'avère être nécessaire pour le bon fonctionnement des organisations internationales.

Parmi les 6 organes principaux prévus par la Charte des Nations Unies, cinq sont des organes restreints ayant une mission particulière. ${ }^{186}$

Il s'agit :

- du Conseil de sécurité spécialisé dans les questions de maintien de la paix et de la sécurité internationale ${ }^{187}$

- du Conseil économique et social chargé des questions économique, sociale, culturelle, éducative et de santé publique ; ${ }^{188}$

- du Conseil de tutelle chargé de veiller sur les progrès de chaque territoire sous tutelle dans les domaines politique, économique et social ;189

- de la CIJ qui est l'organe judiciaire principal des Nations Unies. ${ }^{190}$

- du Secrétariat général ${ }^{191}$ qui est l’administration de l'organisation.

186 Voir larticle 7 de la Charte des Nations Unies.

187 Voir le Chapitre VI, VII et VIII de la Charte des Nations Unies.

188 Voir l'article 62 de la Charte des Nations Unies.

189 Voir l'article 86 et suivant de la Charte des Nations Unies.

190 Voir l'article 92 de la Charte des Nations Unies.

191 Voir le Chapitre XV de la Charte des Nations Unies. 
Au sein de l'UA, cette déconcentration des fonctions a été respectée dans l'Acte constitutif. Parmi les 9 organes prévus par l'Acte constitutif, il y a 5 organes restreints spécialisés avec des missions particulières. Ce sont :

- la Cour de justice, qui est l'organe judiciaire de l'Union ; ${ }^{192}$

- la Commission qui joue le rôle de Secrétariat de l'Union ${ }^{193}$;

- les comités techniques spécialisés ${ }^{194}$ composés des ministres ou de hauts fonctionnaires chargés des secteurs relevant de leurs domaines respectifs de compétence.

- le Conseil économique, social et culturel ${ }^{195}$ qui est un organe consultatif composé de représentants des différends couches socioprofessionnelles des États membres de l'Union, en particulier des jeunes et des femmes.

- le Conseil de paix et de sécurité ${ }^{196}$ chargé de la promotion de la paix, de la sécurité et de la stabilité en Afrique, de la diplomatie préventive et du rétablissement de la paix.

Les exemples peuvent être multipliés, mais ce qu'il convient de retenir ici est le rôle fondamental que jouent les organes restreints dans ladministration des organisations internationales. Ils allègent les missions des organes pléniers. Mais cette déconcentration des organisations internationales par les organes restreints comporte des insuffisances.

\section{PARAGRAPHE II : LES LIMITES A LA DECONCENTRATION DES ORGANISATIONS INTERNATIONALES PAR LES ORGANES RESTREINTS}

La déconcentration des missions des organisations internationales est entravée par l'inflation des organes restreints (A) et la présence d'organes restreints d'apparat dans les organisations internationales (B).

\section{A: L'INFLATION DES ORGANES RESTREINTS DANS LES ORGANISATIONS INTERNATIONALES}

Il est dans la logique des choses que l’Assemblée générale d'une organisation internationale ne puisse délibérer sur tous les points intéressant l’organisation. Sa périodicité, et la multitude propre au forum l'en empêche. La création d'un organe délibérant restreint s'impose donc : constitué par un nombre limité de membres actifs, permanent, celui-ci discute d'une partie des questions liées au fonctionnement de l'organisation, et prend les décisions utiles. ${ }^{197}$

192 Voir l'article 5 paragraphe 1, alinéa (d) de l'Acte constitutif de l'UA.

193 Voir l'article 20 de l'Acte constitutif de l'UA.

194 Voir l'article 14 de l'Acte constitutif de l'UA.

195 Voir l'article 22 de l'Acte constitutif de l'UA.

196 Voir le Protocole relatif à la création du Conseil de paix et de sécurité de l’UA.

197 AUDOIN (Jean-Pierre), L'Autorité internationale des fonds marins (d’après les travaux de la troisième Conférence des Nations Unies sur le droit de la Mer), Thèse d'État, Droit public, Université de Nice, septembre 1979, p.167. 
Mais, les États membres des organisations internationales en créant une multitude dorganes restreints ont vidé la déconcentration fonctionnelle de son sens véritable. Cette situation est symptomatique au sein de l'UA. Dans le cadre de la prévention et du rétablissement de la paix deux organes principaux ont été mis en place. Aux termes de l'article 20 de l'Acte constitutif de l'UA, « est créée une Commission qui est le Secrétariat de l'Union. »" ${ }^{198}$ Dans le protocole du 9 juillet 2002, l'UA a aussi institué le Conseil de paix et de sécurité.

Leurs collaborations dans le domaine de la prévention et du rétablissement de la paix sont prévues aux articles 3.2 (s) du Statut de la Commission et à l'article 7 alinéa 1 du Protocole créant le Conseil de paix et de sécurité.

Larticle 3.2 (s) du Statut de la Commission « dispose quelle apporte un appui opérationnel au Conseil de paix et de sécurité.» ${ }^{199}$ Larticle 7 alinéa 1 du Protocole créant le Conseil de paix et de sécurité précise qu’il exerce ses pouvoirs " conjointement avec le Président de la Commission »200. Quelles analyses pouvons-nous faire de ces deux dispositions?

A priori, la collaboration qui existe entre ces deux organes semble s'expliquer par l'adverbe « conjointement » figurant à l'article 7 du Protocole relatif à la création du Conseil de paix et de sécurité. Ces deux organes agissent de concert dans le cadre de la prévention et du maintien de la paix. Ce qui implique que les deux organes sont censés poser des actions communes en vue de la prévention et du rétablissement de la paix. De ce fait, on pourrait soutenir qu'il n'existe pas de lien de subordination entre ces deux organes.

Toutefois, larticle 10 du Protocole relatif à la création du Conseil de paix et de sécurité bat en brèche cette idée d'absence de subordination entre ces deux organes. En effet, aux termes de cette disposition « le Président de la Commission, sous l'autorité du Conseil de paix et de sécurité et en consultation avec toutes les parties impliquées dans un conflit, déploie tous les efforts et prend les mesures appropriées en vue de la prévention, de la gestion et du règlement des conflits. ${ }^{201} \mathrm{On}$ pourrait déduire de cette disposition le lien de subordination de la Commission au Conseil de paix et de sécurité. La Commission agit uniquement sous l'autorité du Conseil de paix et de sécurité.

Le Protocole relatif à la création du Conseil de paix et de sécurité a institué un autre organe restreint qui intervient aussi dans le domaine de la prévention et du maintien de la paix. Il s’agit du « Groupe des sages ». A ce sujet, l’article 11 alinéa 1 dispose : « Pour venir en aide en appui aux efforts du Conseil de paix et de sécurité et à ceux du Président de la Commission, en particulier dans le domaine de la prévention des conflits, il est crée un Groupe de sage. $»^{202}$ Sa composition est prévue par l'alinéa 1 de l'article 11 en ces termes : "le Groupe de sages est composé de cinq personnalités africaines, hautement respectées, venant de diverses couches de la société et qui ont

198 Voir l'article 20 de l'Acte constitutif de l'UA.

199 Voir l'article 3.2 (s) du Statut de la Commission de l'UA.

200 Voir l’article 7 alinéa 1 du Protocole relatif à la création du Conseil de paix et de sécurité de l'UA.

201 Voir l’article 10 alinéa 1 du Protocole relatif à création du Conseil de paix et de sécurité de l’UA.

202 Voir l’article 11 alinéa 1 du Protocole relatif à la création du Conseil de paix et de sécurité de l'UA. 
apporté une contribution exceptionnelle à la cause de la paix, de la sécurité et du développement sur le continent... $»^{203}$

Cette référence aux Sages est intéressante, en ce sens qu'elle institutionnalise au sein de l'UA les pratiques communautaires africaines. ${ }^{204}$ Mais, ce Groupe de Sages ne vient-il pas accroître le nombre d’organes intervenant dans le domaine de la prévention et du maintien de la paix ? On peut répondre par l'affirmative en ce sens quà la lecture de l'Acte constitutif de l'UA et du Protocole relatif à la création du Conseil de paix et de sécurité, on constate que le Conseil et la Commission ont un statut qui leur permet de s'acquitter aisément de leurs missions. La Commission est un organe intégré, c'est-à-dire un organe composé de nationaux des États membres jouissant sûrement des mêmes qualités de respectabilité que les membres du Groupe des sages. Les organes intégrés sont composés d'individus qui ne sont pas chargés de représenter les États, mais qui sont au service de l'organisation. Ils sont dotés d'un statut qui en principe assure leur indépendance à légard des États membres et ils sont recrutés en fonction de leur compétence. ${ }^{205}$ Le Conseil de paix et de sécurité est un organe interétatique composé de représentants des États membres. En cette qualité, le Conseil de paix et de sécurité garantit pleinement les intérêts des États.

$\mathrm{Au}$ regard de ce qui précède, les rédacteurs de l'Acte constitutif de l'UA auraient pu simplifier le fonctionnement de l'UA en matière de prévention et de maintien de la paix en limitant le nombre d’organes restreints au Conseil de paix et de sécurité et à la Commission. En créant le Groupe de Sages, les fondateurs de l'UA n'ont-ils pas rendu plus compliqué le fonctionnement de cette organisation internationale ? Le nombre pléthorique d'organes restreints au sein de l'UA entraîne généralement des conflits de compétence. En guise d’exemple, nous pouvons mentionner le conflit de compétence qui a opposé le Président de la Commission de l'UA et le Président de la Conférence des Chefs d'État et de gouvernement. Le désaveu public infligé par le Président en exercice de la Conférence, Monsieur Olesegun OBASANJO au Président de la Commission, Alpha Omar KONARE à propos de la décision prise par celui-ci, conformément à l'article 10 du Protocole portant création du Conseil de paix et de sécurité, et sur habilitation expresse de celui-ci, de nommer un médiateur pour aider à résoudre la crise togolaise née des élections d'avril 2005. ${ }^{206}$ Aux termes de l'article 10 alinéa 2 paragraphe (c), « le Président de la Commission, soit peut, de sa propre initiative ou à la demande du Conseil de paix et de sécurité, user de ses bons offices, soit personnellement, soit par l'intermédiaire d’envoyés spéciaux, du Groupe des Sages ou des mécanismes régionaux pour prévenir les conflits potentiels, régler les conflits en cours et promouvoir les initiatives et les efforts de consolidation de la paix et de reconstruction post-conflit. $»^{207}$

Voir l'article 11 alinéa 2 du Protocole relatif à la création du Conseil de paix et de sécurité de l’UA.

DUJARDIN (Stéphanie), «L'Union africaine : objectifs et moyens de gestion des crises politiques et des conflits armées », Op.cit., p.72.

DORMOY (Daniel), Droit des organisations internationales, Dalloz, 1995, p.67.

AHADZI (Koffi), « Réflexions critiques sur l’Union africaine », RBSJA,nº 5 , p.100.

Voir l'article 10 du Protocole relatif à la création du Conseil de paix et de paix de l'UA. 
Ce conflit de compétence entre le Président de la Commission et le Président de la Conférence des Chefs d'État et de gouvernement montre bien selon le Professeur AHADZI que « les Chefs d'État entendent conserver le leadership de l'Union et réduire à peu de chose la marge de manœuvre et les pouvoirs effectifs du Président de la Commission. Ce faisant l'on court le risque de voir renaître l'ex-OUA de ses cendres et de condamner l'UA à être ballotée, telle une girouette, au gré de la volonté des Chefs d'États. $»^{208}$

En plus des conflits de compétence, il y a aussi les coûts budgétaires qu’entraîne la multiplicité des organes restreints. Cette multiplication d’organes restreints peut être interprétée comme une volonté des Chefs d'État africains de varier les sources de l'autorité et de permettre une certaine souplesse dans les prises de décisions et une plus grande efficacité dans la gestion de l'organisation panafricaine. ${ }^{209}$ Mais dans le fond, cette inflation des organes restreints soulève lépineux problème du financement de l'UA. Le financement de la nouvelle Union en général, et de ses organes en particulier reste un sujet de préoccupation car l'OUA n’avait déjà jamais réussi à recouvrer les arriérés dûs par ses membres: ceux-ci devaient, à la fin de 2001, plus de 50 millions de dollars, plus que le budget de la transition de l'OUA à l'UA. ${ }^{210}$

La rationalisation fonctionnelle des activités d'une organisation internationale doit se faire dans une proportion raisonnable, en instituant des organes restreints qui sont nécessaires

208 AHADZI (Koffi), « Réflexions critiques sur l’Union africaine », op.cit., p.100.

209 YAO (Kouassi), «L'Union africaine : OUA bis ou essais transformé ? », op.cit., p.17.

210 L'Acte constitutif de l'UA a prévu 17 organes au lieu de 5 organes qui existaient dans le cadre de la Charte de l'OUA. Interrogé sur cette multiplication des organes, voici la réponse donnée par son Excellence Essy AMARA alors Secrétaire général de cette organisation : «Je pense que c’est parce que les exégètes de ce projet qui veulent peut-être donner une importance économique à l'UA, avec la participation des citoyens, ont pris tous les organes qui son représentatifs de cet objectif. A savoir la Banque africaine, le parlement, le Conseil économique et social, une Cour de justice. Donc un ensemble de structures qui intègrent les questions économiques, les droits de l'homme, la bonne gouvernance...etc. " En outre, sur la question du financement de ces organes, il affirme que : «C'est le grand problème. L’expérience que j’ai tirée de mes fonctions ministérielles, c'est qu’avant de prendre une décision, on envisage d'abord les incidences financières. Et c'est lorsqu'on a les moyens financiers qu’on prend cette décision. Dans le cas d’espèce, c'est ce que je fais ressortir dans les études que j’ai entamées avec le Bureau international du Travail. Par exemple, quel sera le coût du bureau d'un Commissaire ? Combien de directeurs, combien de personnels d'appui, combien de personnels subalternes, combien de voitures, de chauffeurs...etc. Il faut alors multiplier par dix le coût global d'un Commissaire. Comment on va financer tout cela ? Nous avons mis en place un groupe de travail sur la mobilisation des ressources, car je ne crois pas qu'on puisse financer cette Union africaine sur les ressources des États. Déjà avec 4 organes, on est à 53 millions de dollars (plus de 30 milliards de Francs CFA) de déficit. Or quand jextrapole, je conclus qu’on va atteindre au minimum les 100 millions de dollars. Parce que dix Commissaires, plus les organes que nous allons mettre en place, tout cela coûte assez cher. Mais, c'est peut-être le prix de la mutation. Cela dit, j’ai essayé de mobiliser tous ceux qui peuvent apporter des ressources à l'UA, à savoir les hommes d'affaires, les grandes sociétés, les philanthropes, etc. Toutefois, pour moi l'important, c'est de donner une crédibilité à l'UA, car tous ces gens ne s'engageront véritablement que si elle est crédible. " (Voir le quotidien ivoirien Fraternité Matin dans sa parution du lundi 8 juillet 2002 à la page 12) 
pour son bon fonctionnement. ${ }^{211}$ De ce fait, certains organes restreints d'apparat, qui ont en réalité un rôle mineur à jouer dans le fonctionnement des organisations internationales se justifient-ils?

\section{B : LA PRESENCE D'ORGANES RESTREINTS D'APPARAT DANS LES ORGANISATIONS INTERNATIONALES}

Lorsqu'on analyse le fonctionnement de certaines organisations internationales, il est facile de constater la présence d’organes restreints d'apparât. Les organes restreints d’apparat sont ceux qui ne jouent pas un rôle important dans le fonctionnement des organisations internationales. En référence à la classification des institutions qu'avait faite Walter BAGEHOT, pionnier de l'analyse institutionnelle dans le monde anglo-saxon (en dehors des États-Unis) en 1867, une distinction fondamentale doit être opérée entre les institutions dites « d'apparat » et les institutions "defficience ». Alors que les premières ont une valeur symbolique, les secondes participent quotidiennement à la prise de décisions. ${ }^{212}$

Pris dans cette acception, l'organe restreint qui peut illustrer ce point de vue est le Conseil économique et social des Nations Unies. Institué dans le cadre du Chapitre IX de la Charte, ${ }^{213}$ ses compétences les plus importantes sont, en résumé les suivantes :

a) Il peut, en premier lieu, faire ou provoquer des études et des rapports sur des questions internationales dans les domaines économique, social, culturel, de l'éducation, de la santé publique et autres domaines connexes (article 62 de la Charte).

b) Il peut également faire des recommandations en vue d'assurer le respect

c) effectif des droits de l'homme et des libertés fondamentales pour tous.

Mais en pratique dans ces différents domaines d'action, le rôle du Conseil économique et social a été réduit à sa portion la plus congrue par les institutions spécialisées. ${ }^{214}$ Elles ont presque rendu inutile l'existence du Conseil économique et social. Aux termes des articles 57 et 63 de la

211 A titre d’exemple, les nombreux Comités spécialisés prévus par l'article 14 de l'Acte constitutif de l'UA. Ces Comités spécialisés sont:-le Comité chargé des questions d’économie rurale et agricole ;

- Le Comité chargé des questions monétaires et financières ;

- Le Comité chargé des relations commerciales, douanières et d'immigration ;

- Le Comité chargé de l'industrie, de la science et de la technologie, de l'énergie, des ressources naturelles et de lenvironnement ;

- Le Comité chargé des transports, des communications et du tourisme ;

- Le Comité chargé de la santé, du travail et des affaires sociales ;

- Le Comité chargé de l'éducation, de la culture et des ressources humaines.

Malgré cette pléthore d’organes restreints, nous nous posons la question de l'efficacité de l'UA ? Ces nombreux organes restreints doivent justifier leur utilité dans le fonctionnement de l'organisation en réalisant les objectifs pour lesquels ils ont été créés. Dans le cas contraire, ces organes restreints contribueraient à alourdir le mécanisme de fonctionnement de l'organisation.

212 MVELLE (Guy), L'Union africaine, fondements, organes, programmes et actions, op.cit., p.190.

213 Voir le Chapitre IX de la Charte des Nations Unies.

214 ZARB (Antoine H.), Les institutions spécialisées du système des Nations Unies, Pédone, Paris, $198 \mathrm{O}$. 
Charte des Nations Unies, les institutions spécialisées sont créées par accords intergouvernementaux et sont reliés à l'organisation par des accords de liaison. ${ }^{215}$ Une institution spécialisée présente dès lors les caractéristiques suivantes :

1) Elle est créée par un accord entre États,

2) Elle est reliée aux Nations Unies par un accord conclu avec l'ONU. ${ }^{216}$

Les nombreuses institutions spécialisées mises en place par les Nations Unies tendent à diluer le rôle du Conseil économique et social dans l'exécution des missions de l'organisation. Les domaines d'action du Conseil économique et social que nous avons énumérés et ceux des institutions spécialisées coïncident considérablement. Cette coïncidence transparaît clairement dans le contenu de larticle 57 alinéa 2 de la Charte qui dispose que : «les diverses institutions spécialisées créées par accord intergouvernementaux et pourvues, aux termes de leurs statuts, d'attributions internationales étendues dans les domaines économique, social, de la culture intellectuelle et de léducation, de la santé publique et autres domaines connexes... » A partir de quelques exemples d'institutions spécialisées reliées à l'ONU, cette coïncidence pourra être justifiée.

Dans le domaine économique, par l'effet de l'accord approuvé par le Conseil des gouverneurs en septembre 1947 et par l'Assemblée générale en novembre 1947, le FMI est devenu une institution spécialisée des Nations Unies. ${ }^{217}$ Or, en matière économique, comme nous l’avons déjà souligné, ${ }^{218}$ le FMI jouit d'un domaine de compétences très étendues.

Dans le domaine de l'éducation, de la science et de la culture, l'accord conclu entre l'ONU et l'UNESCO, -par lequel elle devient une institution spécialisée-, est adoptée en novembre 1946 par la première Conférence générale de l'UNESCO et approuvée par l'Assemblée générale des Nations Unies. ${ }^{219}$ L'UNESCO se propose, aux termes de l'article 1 de la Convention qui la crée, de « contribuer au maintien de la paix et de la sécurité en resserrant, par l'éducation, la science et le culture, la collaboration entre les nations, afin d'assurer le respect universel de la justice, de la loi, des droits de l'homme et des libertés fondamentales pour tous, sans distinction de race, de sexe, de langue ou de religion, que la Charte des nations Unies reconnait à tous les peuples.» ${ }^{220}$

L'accord régissant les rapports de l'OMS avec l'ONU est approuvé par l'Assemblée générale le 15 novembre 1964 et est entré en vigueur le 10 juillet 1948, date de son approbation par l'Assemblée de l'OMS. ${ }^{221}$ Aux termes de l'article $1^{\text {er }}$ de sa Constitution, l'OMS a pour but « d'amener

\footnotetext{
215 Voir les articles 57 et 63 de la Charte des Nations Unies.

216 DIEZ DE VELASCO VALLEJO (Manuel), Les organisations internationales, op.cit., p.339.

217 Ibid, p.394.

218 Il s'agit du point concernant la pluralité des missions des organisations internationales. Le domaine de compétence très étendu du FMI réduit considérablement le rôle du Conseil économique et social de l'ONU.

219 DIEZ DE VELASCO VALLEJO (Manuel), Les organisations internationales, op.cit., p.359.

220 Ibid.

$221 \quad$ Ibid, p.354.
} 
tous les peuples au niveau de santé le plus élevé ».22 Pour atteindre son but, elle exerce les fonctions suivantes, qui sont énumérées à l'article 2 :

a) Agir en tant qu’autorité directrice et coordinatrice, dans le domaine de la santé, des travaux ayant un caractère international ;

b) Etablir et maintenir une collaboration effective avec les Nations Unies, les institutions spécialisées, les administrations gouvernementales de la santé, ainsi que telles autres organisations qui paraîtraient indiquées.

c) Aider les gouvernements, sur leur demande, à renforcer leurs services de santé..... ; 223 Les domaines d'action des institutions spécialisées couvrent presque ceux du Conseil économique et social. Or, elles sont autonomes à l'égard de l'ONU. ${ }^{224}$ Cette autonomie se justifie puisque les manifestations de volonté des institutions spécialisées leur sont imputables directement et non à l'ONU. ${ }^{225}$ Elle est établie, dès lors que dans plusieurs domaines importants il n’y a pas d'identité :

a) Les ordres juridiques respectifs sont clairement séparés. L'ONU a son ordre juridique propre, qui est distinct de celui de chacune des institutions spécialisées ;

b) Les membres des Nations Unies et ceux des institutions spécialisées ne sont pas nécessairement les mêmes, il est possible d'être membre de l'ONU sans être membre des institutions spécialisées, et inversement ;

c) Tant les organes que les fonctionnaires des institutions spécialisées sont distincts dans tous les cas des Nations Unies. 226

Au regard de la connexité du domaine d'action du Conseil économique et social et des institutions spécialisées et de l'autonomie de ces institutions vis-à-vis du Conseil économique, des conséquences peuvent être tirées relativement au rôle de cet organe restreint dans le système des Nations Unies. Compte tenu de l'importance des institutions spécialisées, qui ont le statut d’organisation internationale, les États auront tendance à recourir à ceux- ci plutôt quà un organe restreint. C’est ainsi que les grandes questions économiques vitales continuent à être gérées en dehors de l'ONU, notamment à travers le bilatéralisme ou par le truchement d’organisations

222 Ibid, p.355.

223 Ibid.

224 DIEZ DE VELASCO VALLEJO (Manuel), Les organisations internationales, op.cit., p.343. Mais, l'auteur précise que « les institutions spécialisées sont autonomes à légard de l'ONU, sous réserve des rapports de coopération et de contrôle [... ] Ces rapports de coopération et de contrôle sont les suivantes :

1) Des représentants des institutions spécialisées ont la possibilité, par le biais d’accords, de participer sans droit de vote, aux délibérations du Conseil économique et social ;

2) La possibilité déchanger des rapports et des documents ;

3) Une coopération avec le Conseil économique et social, dans le but de fournir au Conseil de sécurité et à l'Assemblée générale les informations ainsi que l'assistance que ceux-ci sont susceptibles de lui demander pour des questions relatives au maintien de la paix et de la sécurité internationales. » Ibid, p.344. 
dominées ou créées par les pays industrialisés (FMI, OMS, UNESCO...etc). ${ }^{227}$ Dans ces conditions, le Conseil économique et social est destiné à devenir un organe restreint sans aucune importance réelle dans le fonctionnement de l'ONU. Or, le Conseil économique et social doit être l'instance de haut niveau chargée des questions de coopération au développement, qui examine les tendances de la coopération internationale en faveur du développement, qui favorise la cohérence des activités de développement des différents acteurs et qui rapproche les volets normatifs et opérationnels de l'activité de l'organisation. ${ }^{228}$ Mais dans les faits, le Conseil économique et social est réduit au statut d' « organe restreint d'apparat » par les rédacteurs de la Charte de l'ONU.

Les organes restreints contribuent considérablement au fonctionnement efficace des organisations internationales. Cependant, leurs apports ne se limitent pas à ce niveau. Au plan politique, ils jouent aussi un rôle important.

227 HAZBI (Aziz), ONU et ordre mondial : reformer pour ne rien changer, L'Harmattan, Paris, 2005, p.66.

228 NOVOSSELOFF (Alexandra), «L'ONU ou la réforme perpétuelle », AFDI, 2004, p.542. 


\section{CHAPITRE II : LES APPORTS AU PLAN POLITIQUE DES ORGANES RESTREINTS AU FONCTIONNEMENT DES ORGANISATIONS INTERNATIONALES}

Les raisons politiques qui justifient la création des organes restreints sont déterminantes. ${ }^{229}$ A ce niveau, leurs contributions au bon fonctionnement des organisations internationales se manifestent d'abord par la mise place d'une démocratie représentative (Section I) et par la complémentarité qui existe entre ceux-ci et les organes pléniers. (Section II). 
Nous analyserons d'une part le contenu de la notion de démocratie représentative dans les organes restreints (Paragraphe I) et sa portée dans les organisations internationales d'autre part (Paragraphe II).

\section{PARAGRAPHE I : LE CONTENU DE LA NOTION DE DEMOCRATIE REPRESENTATIVE DANS LES ORGANES RESTREINTS}

Lanalyse de la notion de démocratie représentative implique nécessairement sa clarification juridique (A) avant de poser ses conditions de mise œuvre (B).

\section{A : LA CLARIFICATION DE LA NOTION DE DEMOCRATIE REPRESENTATIVE}

Etymologiquement, la démocratie est perçue comme le pouvoir du peuple, par le peuple et pour le peuple. Il en résulte que le peuple est le titulaire du pouvoir politique. ${ }^{230}$

Dans le cadre des organisations internationales, «l'État citoyen de l'organisation », ${ }^{231}$ institue également une représentation démocratique dans les organes restreints. Pour mieux comprendre ce système, il faut établir une comparaison avec le système de représentation qui prévaut dans les organes pléniers. Dans les organes pléniers le système de représentation est la

230 La démocratie est le régime politique dans lequel le peuple est souverain. La formule d’Abraham LINCOLN, « le gouvernement du peuple par le peuple et pour le peuple » est l'une des définitions canoniques couramment reprises, ainsi qu’en témoigne son introduction dans la Constitution de 1958 de la cinquième République française. Cette définition est proche du sens étymologique du terme démocratie, du grec ancien « démokratia ", souveraineté du peuple, de « dêmos » (peuple) et Kratos (pouvoir) ; le peuple renvoyant à la notion plus restrictive de citoyens (la citoyenneté nétant pas forcément donnée à toute la population). Cependant cette définition reste susceptible d'interprétations différentes, aussi bien quant à la qualification de la souveraineté populaire que pour son application pratique. Ce qui apparaît clairement au regard de la diversité des régimes politiques qui se sont revendiquées et qui se revendiquent comme démocratie. Ainsi, aujourd'hui encore, il n'existe pas de définition communément admise de ce qu’est ou doit être la démocratie. De façon générale, un gouvernement est dit démocratique par opposition aux systèmes monarchiques d'une part, où le pouvoir est détenu par un seul individu, et d’autre part aux systèmes oligarchiques où le pouvoir est détenu par un groupe restreint d'individus. On peut aussi définir la démocratie par opposition à la dictature ou la tyrannie, mettant ainsi l'accent sur la possibilité pour le peuple de contrôler ses dirigeants, et de les évincer sans devoir recourir à une révolution. Par ailleurs, le terme de démocratie ne se réfère pas uniquement à des formes de gouvernement, mais peut désigner une société ayant pour valeur la liberté et l'égalité ou de manière plus générale encore un ensemble de valeurs, d'idéaux et de principes politiques, sociaux ou culturels. Le terme de démocratie peut aussi servir à qualifier le fonctionnement de tout corps ou organisation sociale (organismes publics ou privés, associations ou entreprises), le plus souvent par le biais du qualificatif de démocratique. Cela signifie alors que ce fonctionnement repose sur légalité des membres du groupe, sur des procédures de délibérations, ou encore de votes et/ ou d'élections. 
démocratie directe interétatique. ${ }^{232}$ Concrètement, elle s'exprime par la confusion des gouvernants et des gouvernés. ${ }^{233}$ Précisément, en raison du principe dégalité, les États membres sont en même temps les gouvernants et les gouvernés dans les organes pléniers. La démocratie directe trouve son expression la plus achevée à l'Assemblée générale des Nations Unies, où les États membres sont tout à la fois les auteurs de la Charte et des résolutions qu'ils votent au cours des sessions de cet organe et les destinataires de ces normes élaborées par eux. ${ }^{234}$

En revanche, dans les organes restreints prévaut le système de démocratie représentative. ${ }^{235}$ Par ce système, les États membres d'une organisation internationale donnent un mandat politique à un groupe d'États, -qui sont élus ou permanents-, afin de les représenter dans les organes restreints. Le Professeur DUPUY soutient que « ... les États [...] choisissent certains d'entre eux pour les représenter, de la même manière que, dans lordre interne, les citoyens groupés dans la nation désignent certains représentants, pris dans son sein [...] Cette analyse n'a rien d'irréel ; elle vise notamment la SDN et l'ONU, qui à côté de tentatives de démocratie directe au niveau des Assemblées générales, ont instauré un système représentatif avec la constitution dorganes restreints. ${ }^{236}$ Dans toutes les organisations internationales, les membres des organes restreints sont supposés représenter tous les États membres. Au regard de la Charte de l'ONU, le seul organe statutairement habilité à agir est le Conseil de sécurité. ${ }^{237}$ De ce fait, lorsque celui-ci agit au nom et pour le compte de l'ensemble des membres des Nations Unies, il ne se comporte pas comme le ferait un représentant dont la volonté serait représentative de celles des membres ; c'est l'ensemble

DUPUY (René-Jean), La communauté internationale, entre le mythe et l'histoire, Paris, Economica, 1986, p.48.

Ibid. Dans la démocratie directe, le pouvoir est exercé directement par les citoyens, sans l'intermédiaire des organes représentatifs. L'idée de démocratie directe se rapporte selon les penseurs à différentes conceptions de l'exercice direct de la souveraineté par le peuple. De même selon les lieux et les époques, la démocratie directe a désigné différentes formes de gouvernements ou d'associations politiques, dans lesquels la population décide des lois. Si aujourd'hui le terme de démocratie renvoie à l'idée de gouvernements représentatifs, il fut longtemps associé à celle de démocratie directe, notamment en référence à celle de la démocratie athénienne : les citoyens réunis en assemblée y décidaient des lois, les magistrats aux fonctions administratives et exécutives étaient tirés au sort, et les magistrats dont la fonction nécessitait une expertise étaient élus et révocables par les citoyens. Différents exemples de démocratie directe jalonnent l'histoire, généralement dans le cadre d'un exercice local du pouvoir. Ce fut le cas au XVIIe siècle en Nouvelle- Angleterre, au travers des « town meeting », où la population des communes réunie en assemblée décide des lois, impôts et budgets.

234 Ibid. En dehors de l'Assemblée générale de l'ONU, la Conférence des Chefs d'État et de gouvernement de l'UA, l'Assemblée générale de l'Autorité des fonds marins ...etc incarnent aussi la démocratie directe interétatique au sein des organisations internationales.

235 La démocratie représentative appelée aussi la «démocratie délégative » est l'une des formes de la démocratie dans la quelle les citoyens expriment leur volonté par l'intermédiaire de représentants élus à qui ils délèguent leur pouvoirs. Ces élus qui représentent la volonté générale, votent la loi et contrôlent éventuellement le gouvernement.

236 DUPUY (René-Jean), "L'organisation internationale et l’expression de la volonté générale », RGDIP, 1957, p.551. 
des membres des Nations Unies qui est réputé agir par l'intermédiaire du Conseil de sécurité, car lorgane statutaire déclare, non pas sa volonté propre, mais celle du groupe qui veut par lui. ${ }^{238}$.

Le fondement de la démocratie représentative dans les organisations internationales est le mandat donné par les États membres aux membres des organes restreints. Mais, une question assez pertinente mérite dêtre posée : quelle est la nature du mandat dont jouissent les membres des organes restreints ? Sagit-il d'un mandat représentatif ou impératif ? ${ }^{239}$ En principe, les membres des organes restreints sont réputés libres de tout mandat impératif. ${ }^{240}$ Le principe représentatif a ainsi essentiellement une fonction unificatrice; il tend à affranchir lélu de la pression des intérêts spéciaux ou locaux pour le consacrer à la poursuite de l'intérêt général. ${ }^{241}$ Chaque membre de l'organe restreint détient un mandat qu'il exerce librement au nom et pour le compte de l'ensemble des États membres. En général, cet organe n’existe que pour la recherche et la satisfaction de l'intérêt général. C’est pour y participer en commun que les États y sont entrés; c'est dans son sein qu'ils sélèvent ensemble à une conception du bien commun. ${ }^{242}$

Cependant, ce principe semble être battu en brèche par la situation particulière des membres permanents dans les organes restreints. ${ }^{243}$ Leur statut particulier fait d'eux des superreprésentants : leur accord est interprété par les Actes constitutifs comme l'expression de la volonté générale. ${ }^{244}$

La notion de démocratie représentative participe d'une volonté intégrée, celle d'un ensemble qui confie à certains agents le pouvoir de vouloir pour lui. ${ }^{245}$ Mais dans les faits, quelles sont les conditions qui président à sa mise en œuvre?

\section{B. LES CONDITIONS DE MISE EN CEUVRE DE LA DEMOCRATIE REPRESENTATIVE}

La mise en œuvre de la démocratie représentative dans les organes restreints suppose réunie certaines conditions. La première est relative à la délégation de compétence des membres des organes pléniers aux membres des organes restreints (1). Quant à la seconde, elle porte sur la légitimation des pouvoirs des membres des organes restreints par l'ensemble des États (2)

238 CARRÉ DE MALBERG (Raymond), Contribution à la théorie générale de l'État, Tome II, 1922, p.281 et 282.

239 Il faut le rappeler, un mandat est dit impératif lorsque la révocation de lélu est en permanence possible. En revanche, dans le mandat représentatif, les représentants ne peuvent être révoqués en cours de mandat et ils n’ont pas d’obligation de rendre compte à leurs électeurs.

240 DUPUY (René-Jean), "L'organisation internationale et l’expression de la volonté générale », op.cit., p.552.

$241 \quad$ Ibid, p.559.

242 DUPUY (René-Jean), «L'organisation internationale et l'expression de la volonté générale », op.cit., p.537.

243 La notion de membres permanents sera explicitée dans le titre II de cette première partie de notre thèse.

244 DUPUY (René-Jean), « Lorganisation internationale et l'expression de la volonté générale », op.cit., p.553.

245 Ibid, p.532. 


\section{1/ La délégation de compétence des membres des organes pléniers aux organes restreints.}

$\mathrm{Au}$ sens du droit interne, la délégation de pouvoirs consiste pour le titulaire d'une compétence, le délégant, à transmettre, pour un temps, l'exercice de cette compétence à l'autorité qui lui est subordonnée, le délégataire ou le délégué. ${ }^{246} \mathrm{Il}$ s’ensuit que dans la technique de la délégation, l'autorité qui délègue ses pouvoirs est une autorité hiérarchiquement supérieure et cette délégation s'effectue dans un temps bien déterminé.

Dans les organisations internationales, la délégation de pouvoirs s'opère entre les organes pléniers et les organes restreints. ${ }^{247}$ L'ensemble des États membres regroupés dans les organes pléniers délèguent leurs compétences aux membres des organes restreints. Cette délégation est souvent prévue et organisée par la charte constitutive des organisations internationales.

Dans la Charte de l'ONU, les États membres de l'organisation mondiale ont à travers les articles 24 et 61 délégué respectivement leurs compétences au Conseil de sécurité et au Conseil économique et social. Larticle 24 paragraphe 1 dispose qu' " afin d'assurer l'action rapide et efficace de l'organisation, ses membres confèrent au Conseil de sécurité la responsabilité principale du maintien de la paix et la sécurité internationale et reconnaissent qu’en s'acquittant des devoirs que lui impose cette responsabilité le Conseil de sécurité agit en leur nom... ${ }^{248}$ Cette disposition contient l'affirmation d’une véritable délégation de pouvoirs consentie par les États. ${ }^{249}$

Le Professeur DEGNI-SEGUI estime que « l'objet de la délégation ne semble pas à priori poser problème. A s'en tenir à la lettre du texte, c'est la fonction primordiale du maintien de la paix qui est transférée de la sphère de compétence de chacun des États à celle du Conseil de sécurité.

DEGNI-SEGUI (René), Droit administratif général, Tome 2 : L’action administrative, $3^{\text {ème }}$ édition, Abidjan, CEDA, 2003, p.516.

247 Toutefois, une équivoque doit être levée : les organes pléniers qui délèguent leurs pouvoirs ne sont pas, dans toutes les organisations internationales, hiérarchiquement supérieurs aux organes restreints. A titre d’exemple, la délégation de pouvoirs des États membres de l’Assemblée générale de l’ONU au Conseil de sécurité.

248 Voir l'article 24 de la Charte de l'ONU.

249 DEGNI-SEGUI (René), "Commentaire de l'article 24 paragraphe 1 et 2 de la Charte des Nations Unies ", in COT Jean-Pierre et PELLET (Alain) (dir.), La Charte des Nations Unies, commentaire article par article, op.cit.,p. 450. Il est important de souligner que le style utilisé par les rédacteurs de la Charte est sans équivoque sur la volonté des États membres siégeant à l’Assemblée générale de déléguer leurs compétences au Conseil de sécurité. Cette volonté transparaît clairement à travers les verbes « conférer », « reconnaître » et " agir ». Par le verbe «conférer », les États membres octroient leurs compétences aux 15 membres du Conseil de sécurité. Il s'agit d'un acte de volonté de l’ensemble des membres des Nations Unies. Aussi, cette volonté se matérialise par le fait qu'ils « reconnaissent » que tous les actes pris par le Conseil de sécurité le sont en leur nom. De ce fait, le Conseil de sécurité devient l'organe qui incarne l'intérêt général de l’ensemble des États membres. Mais au-delà des textes, la question qui se pose est de savoir si le Conseil de sécurité s’acquitte effectivement de ses missions d'intérêt général ? Cette question appelle nécessairement une réponse dialectique. En effet, aux termes de l'article 24, le Conseil de sécurité a la responsabilité principale du maintien de la paix et de la sécurité internationale. Au regard de cette disposition, le Conseil apparait comme un instrument de pacification dans les relations internationales. Mais évidemment, une telle mission néchappe pas aux contradictions de la société internationale. Ainsi, la mission d'intérêt général du Conseil de sécurité est entravée par les conflits d'intérêts existant entre les États. 
Cette fonction est transmise avec les pouvoirs y afférents. Cela revient à dire qu'il s'agit en fait d'une délégation de pouvoirs. Mais, en poussant plus loin la réflexion l'on aura aucune peine à découvrir que la délégation porte en définitive sur la souveraineté. La fonction du maintien de la paix constitue, en effet, une prérogative de puissance publique, qui participe de la souveraineté de l'État. ${ }^{250}$ Ce point de vue est juste. Cependant, il paraît très excessif dans la mesure où les États membres en décidant de devenir membre de l'ONU, accepte d'abandonner une partie de leur souveraineté au profit de l'organisation.

Larticle 61 alinéa 1 de la Charte dispose que "le Conseil économique et social se compose de cinquante-quatre membres de l'organisation des Nations Unies, élus par l'Assemblée générale... $»^{251} \mathrm{~A}$ travers l'élection des membres de cet organe restreint, les membres de l'Assemblée générale délèguent leurs pouvoirs. Cette délégation de pouvoirs se perçoit nettement à travers l'article 60 de la Charte qui précise que "l'Assemblée générale, et sous son autorité, le Conseil économique et social qui dispose à cet effet des pouvoirs qui lui sont attribués aux termes du Chapitre X, sont chargés de remplir les fonctions de l'organisation énoncées au présent chapitre. $»^{252}$ Quoique le Conseil soit lui-même un des organes principaux de l'organisation et soit destiné à se charger de lessentiel du travail dans les domaines économique et social, il agit toujours sous l'autorité de l’Assemblée. ${ }^{253}$ De ce fait, l’Assemblée délègue ses compétences en matière économique et social au Conseil économique et social.

Au regard de l'Acte constitutif de l'UA, la Conférence des Chefs d'État et de gouvernement a délégué ses pouvoirs au Conseil de paix et de sécurité et à la Commission. Le fondement juridique de cette délégation de pouvoirs se perçoit au paragraphe 2 de l'article 9 de l'Acte constitutif. Aux termes de cette disposition, «la Conférence peut déléguer certains de ses pouvoirs et attributions à l'un ou l'autre des organes de l'Union. " ${ }^{254}$ C'est logiquement en application de cette disposition que le Conseil de paix et de sécurité et la Commission ont reçu un titre de compétence pour agir au nom et pour le compte de toute l'organisation.

Larticle 3 paragraphe 2 alinéa a) du Statut de la Commission de l'UA souligne cette intention des fondateurs de lorganisation en ces termes :

«la Commission :

Représente l'Union et défend ses intérêts, sous l'autorité et sur le mandat de la Conférence... ${ }^{255}$

\footnotetext{
250 DEGNI-SEGUI (René), « Commentaire de l'article 24 paragraphe 1 et 2 de la Charte », op.cit., p.450.

251 Voir l'article 61 de la Charte de l'ONU.

252 Voir l'article 60 de la Charte de l'ONU.

253 BAUCHARD (Denis), "Commentaire de l'article 60 de la Charte des Nations Unies », in COT (Jean-Pierre) et PELLET (Alain) (dir.), La Charte des Nations Unies, commentaire article par article, op.cit., p.930.

254 Voir l'article 9 de l'Acte constitutif de l'UA.

255 Voir l'article 3 du Statut de la Commission de l’UA.
} 
Les membres de la Commission, composée d'un Président, d'un Vice-président et de huit Commissaires ne sont que des mandataires de l'organisation. ${ }^{256} \mathrm{~A}$ ce titre, ils s'acquittent du mandat qui leur a été confié en faisant prévaloir les valeurs d'intégrité, de transparence, de bonne gouvernance, ainsi que de solidarité et d’engagement ferme pour la cause de l'unité africaine. ${ }^{257}$

A travers l'article 7 paragraphe 2 du Protocole relatif à la création du Conseil de paix et de sécurité, les Etats ont donné mandat aux membres de cet organe d’agir pour leur compte. Cette disposition stipule : "Les États membres reconnaissent qu'en s'acquittant de ses devoirs aux termes du présent Protocole, le Conseil de paix et de sécurité agit en leur nom... $»^{258}$ Cette disposition calquée sur le modèle de l'article 24 de la Charte de l'ONU relatif à la délégation des pouvoirs de l'ensemble des membres de cette organisation au Conseil de sécurité, ne nécessite pas de commentaires particuliers.

La délégation des pouvoirs de l'ensemble des États membres est la première condition qui préside à l'institution d'un système de démocratie représentative dans les organisations internationales incarnée par les organes restreints. Toutefois, un autre élément indispensable qui contribue à son efficacité mérite dêtre souligné. Il s’agit de la légitimation des membres des organes restreints par l'ensemble des États membres.

\section{2/ La légitimation des membres des organes restreints par les États membres}

La légitimation des membres des organes restreints par l'ensemble des États membres des organisations internationales constitue aussi une condition essentielle de la réalisation de la démocratie représentative dans les organes restreints. De façon générale, la légitimation doit être entendue comme lacte par lequel on accorde la légitimité à un individu. Quant à la légitimité, elle est la «qualité du pouvoir dont l'acceptation se fonde non sur la coercition comme ressource première, mais sur le consentement réputé libre de la population qui s'y trouve soumise [...] Elle constitue lélément de portée sociale et intime à la fois qui fait accepter volontairement par les gouvernés l’autorité des gouvernants. ${ }^{259}$

La légitimation des membres des organes restreints implique l'acceptation par l'ensemble des États membres de leur représentation dans les organes restreints par certains États. Comme le

256 Le Président est le Chef exécutif de la Commission, représentant légal de l’Union et ordonnateur de la Commission (article 7 du Statut). Le Vice-président est responsable devant le Président, l'assiste dans l'exercice de ses fonctions, exerce tous les pouvoirs d'attribution que lui délègue le Président, et surtout assume la responsabilité de l'administration et des finances de la Commission. Les Commissaires quant à eux, élus par le Conseil exécutif sur proposition de deux candidats par région, dont une femme pour chaque portefeuille, sont chargés de la mise en œuvre de tous les programmes, politiques et décision concernant leur portefeuille respectif. Ils sont responsables devant le Président. 
souligne à juste titre le Professeur René-Jean DUPUY « dans le superétatisme représentatif ainsi conçu, les États se groupent dans un ensemble, détenteur d'une volonté qui ne peut être que la volonté générale... ${ }^{260}$ Les États membres acceptent librement de se faire représenter dans les organes restreints par d’autres États. C’est cette libre acceptation qui confère une légitimité aux membres des organes restreints.

Toutefois, la légitimité des membres de certains organes restreints se présente comme une «légitimité contradictoire». ${ }^{261}$ Le caractère contradictoire de cette légitimité découle du fait qu’elle fait apparaître une «dualité de régime» entre les membres de ces organes restreints. Certains États «se voient reconnaître une mission de représentation à titre permanent; cependant que d'autres sont élus par rotation.» ${ }^{262}$

Les États qui jouissent d'une mission de représentation permanente témoignent d'«une légitimité classique, résultant de la puissance sur l'échiquier international.» ${ }^{263} \quad$ Peuvent être cités en exemple, les 5 membres permanents du Conseil de sécurité de l'ONU, ${ }^{264}$ les 10 États ayant la puissance industrielle la plus considérable au Conseil d'administration de l'OIT.

En revanche, les États élus par rotation dans les organes restreints incarnent une «légitimité démocratique» dans la mesure où ils peuvent être considérés comme les représentants de la société interétatique mondiale. ${ }^{265}$ Tel est le cas des 10 membres non permanents du Conseil de sécurité de l'ONU élus pour deux ans par l’Assemblée générale, et aussi les 18 États membres du Conseil d’administration de l'OIT élus par la Conférence des Chefs d'État et de gouvernement. Le fait que ces États soient élus donne une onction démocratique à leur représentation dans les organes restreints.

260 DUPUY (René-Jean), « L'organisation internationale et l'expression de la volonté générale », op.cit., p.551.

261 MOREAU DEFARGES (Philippe), Lordre mondial, op.cit., p.71.

262 DUPUY (René-Jean), « L’organisation internationale et l’expression de la volonté générale »,op.cit., p.551 et 552. Ce point relatif au statut de membres permanents et de membres provisoires des organes restreints sera plus amplement abordé dans le chapitre II de cette première partie de notre thèse.

263 MOREAU DEFARGES (Philippe), Lordre mondial, op.cit., p.71.

264 Dans le cadre de l'ONU, la légitimation des membres permanents apparaît très clairement dans les déclarations faites par les grandes puissances elles-mêmes et aussi par les petits États. Ainsi, à la Conférence de San Francisco, le point 9 de la déclaration des quatre puissances invitantes l’exprime en ces termes : «Etant donné les responsabilités primordiales des membres permanents, on ne peut s'attendre, dans létat actuel des conditions du monde, à ce qu'ils agissent dans un domaine aussi grave que le maintien de la paix et de la sécurité internationales en exécution d'une décision à laquelle ils n’ont pas donné leur adhésion... » Bien plus, les puissances moyennes et petites reconnaissent et admettent le principe de l'inégalité et l'on peut lire dans leurs suggestions et leurs mémorandums de Dumbarton Oaks des passages aussi significatifs que celui-ci : «Le fait que les grandes puissances jouissent d'une situation privilégiée est sans doute normale et justifiée par les responsabilités plus grandes qu'elles assument dans le maintien de la paix. » (Voir BOUTROS-GHALI, « Le principe dégalité des États et les organisations internationales ", op.cit., p.12). Ibid. 
En marge des tentatives de démocratie directe au niveau des Assemblées générales, ont été instauré un système représentatif avec la constitution dorganes restreints. ${ }^{266}$ Mais quelle est la portée réelle de ce système au sein des organisations internationales?

\section{PARAGRAPHE II : LA PORTEE DE LA DEMOCRATIE REPRESENTATIVE DANS LES ORGANISATIONS INTERNATIONALES}

L’analyse de la portée de la démocratie représentative dans les organes restreints doit se faire à un double niveau. S'il est vrai que la démocratie représentative joue un rôle important dans le fonctionnement des organisations internationales (A), il n’en demeure pas moins vrai quelle recèle de nombreuses lacunes (B).

\section{A : LE ROLE DE LA DEMOCRATIE REPRESENTATIVE DANS LE FONCTIONNEMENT DES ORGANISATIONS INTERNATIONALES}

La démocratie représentative incarnée par les organes restreints joue un rôle inestimable dans le fonctionnement des organisations internationales.

Pour le Professeur René-Jean DUPUY, « elle marque un progrès de la cohésion des États. Ceux-ci admettent qu'ils constituent, par leur groupement, une entité nouvelle dont la volonté peut être exprimée par quelques uns d’entre eux, choisis pour les représenter $»{ }^{267}$ Les États prennent l'engagement de conférer aux membres des organes restreints une responsabilité politique qu'ils ne peuvent assumer en raison de leur nombre pléthorique.

Dans les organisations internationales à vocation politique, cet engagement politique des États membres est souvent matérialisé par une disposition de l'acte constitutif. La Charte de l'ONU en son article 25 est un exemple remarquable. En effet, aux termes de cette disposition « les membres de l'organisation conviennent d’accepter et d’appliquer les décisions du Conseil de sécurité conformément à la présente Charte.» Le but de l’article 25 est de mettre en pratique l’engagement des États membres « de conférer au Conseil de sécurité la responsabilité principale du maintien de la paix et de la sécurité internationales.» ${ }^{268}$ En outre, dans la même perspective, l’article 7 alinéa 3 du Protocole relatif à création du Conseil de paix et de sécurité de l’UA traduit la volonté politique

\footnotetext{
266 DUPUY (René-Jean), « Les organisations internationales et l'expression de la volonté générale », op.cit., p.551

267 DUPUY (René-Jean), Le droit international, Que sais-je ? n¹060, op.cit., p.81.

268 SUY (Erick), " Commentaire de l'article 25 de la Charte », in COT (Jean-Pierre) et PELLET (Alain) (dir.), $\mathrm{La}$ Charte des Nations Unies, commentaire article par article, op.cit.,p.477.
} 
des États membres de conférer à cet organe restreint une responsabilité politique majeure dans la mise en œuvre des actions visant la sécurité collective sur le continent. ${ }^{269}$

Dans les organisations internationales à vocation technique, l'institution d'organes restreints exprime aussi la cohésion des États membres. Ceux-ci sont représenter dans les organes restreints par d’autres États qu'ils ont désignés. ${ }^{270}$ De ce fait, les 56 membres du Conseil d'administration de l'OIT et les 36 membres du Conseil de l'Autorité des fonds marins sont choisis par l'ensemble des États membres afin de mettre en œuvre un aspect particulier de la politique de ces organisations internationales.

«L’efficacité du nombre» ${ }^{271}$ semble justifier le recours à la démocratie représentative dans les organisations internationales. Elle se fonde sur des exigences fonctionnelles. Mais, ce système comporte de nombreuses lacunes qu'il convient de relever.

\section{B : LES LACUNES DE LA DEMOCRATIE REPRESENTATIVE DANS LES ORGANISATIONS INTERNATIONALES}

En dépit de son apport inestimable dans le fonctionnement des organisations internationales, la démocratie représentative comporte de nombreuses lacunes. En principe les États qui siègent dans les organes restreints doivent justifier la confiance qui leur est faite par les autres, se placer au point de l'intérêt général et non se considérer comme des privilégiés, pouvant faire prévaloir, grâce à ce siège dans l'organe restreint leurs intérêts particuliers. ${ }^{272}$

La question de l'intérêt général se trouve au centre de la représentation des États dans les organes restreints. Cependant, les membres des organes restreints ont-ils la même perception de l'intérêt général ? En fait, répond le Professeur René-Jean DUPUY, « les États machines faites pour défendre des intérêts nationaux, auront toujours du mal à se trouver au niveau de l'intérêt général ou à s'y maintenir. " ${ }^{273}$ L'observation de la pratique des États au sein des organes restreints des organisations internationales nous donne de constater que la recherche de l'intérêt général est loin de constituer leur préoccupation essentielle. Le cas des membres du Conseil économique et social et du Conseil de sécurité de l'ONU peut nous aider à mieux comprendre cette situation.

Par la création du Conseil économique et social, les auteurs de la Charte des Nations Unies semblent avoir compris l’importance des menaces non militaires à la sécurité des États. Celles-ci

269 Il faut préciser que l’article 7 alinéa 2 du Protocole relatif à la création du Conseil de paix et de sécurité de l’UA est rédigé dans les mêmes termes que l'article 25 de la Charte des Nations Unies : "Les États membres conviennent d'accepter et d’appliquer les décisions du Conseil de paix et de sécurité, conformément à l'Acte constitutif».

270 Mais, à la différence des organisations internationales à vocation politique, dans les organisations à vocation technique, il n'y a pas une disposition expresse de l'Acte constitutif qui exprime formellement cette volonté des États membres de se faire représenter dans les organes restreints.

271 DUPUY (René-Jean), «L'organisation internationale et l'expression de la volonté générale », op.cit., p.529.

272 DUPUY (René-Jean) Le droit international, Que sais-je ? nº 1060, op.cit., p.81.

273 Ibid., p.82. 
ont été évoquées au Chapitre IX qui parle des menaces qui peuvent surgir « dans les domaines économique, social, de la santé publique comme dans la violation des droits de l'homme. » ${ }^{274} \mathrm{De}$ ce fait, le Chapitre $\mathrm{X}$ a institué le Conseil économique et social pour veiller à ce que l'humanité ne parvienne à la situation actuelle, où un désordre économique aggravé risque de devenir « la source directe de conflits entre États ou de conflits interne, qui à tout instant, peuvent se propager à travers les frontières. ${ }^{275}$

Toutefois, les Chapitres IX et $\mathrm{X}$ de la Charte ont été victimes des dures réalités économiques : les pays industrialisés ont laissé séclipser le rôle du Conseil économique et social et la loi du plus fort s'est imposée dans les relations économiques internationales. ${ }^{276}$ Les questions ayant une importance majeure pour les membres des Nations Unies, et qui relèvent de la compétence de cet organe restreint sont traitées en fonction des intérêts des différents groupes d'États qui la composent.

Quant au Conseil de sécurité, sa mission fondamentale est d'appliquer le système de sécurité collective mis en place par les auteurs de la Charte. Il est le « gardien » de la paix et de la sécurité internationales. Cependant, cette quête de l'intérêt général est entravée par les rapports de force politique, économique et militaire qui prévalent entre ses membres permanents et non permanents. Ainsi, les récentes guerres menées par une coalition d'États (États-Unis, RoyaumeUni, France, Espagne...etc) contre l'Irak le 3 septembre 1996 semblent prouver que le mécanisme de sécurité collective est ignoré lorsque les intérêts des États occidentaux sont directement en cause dans un conflit armé. ${ }^{277}$ Se fondant sur la violation de la résolution 688 qui exigeait que l'Irak mette fin à la répression contre les populations Kurdes, et facilite l'accès de l'assistance humanitaire aux dites populations, ces États ont attaqué l'Irak de façon unilatérale et sans aucune autorisation du Conseil de sécurité. Or, comme le fait remarquer le Professeur DJIENA WEMBOU, « la résolution 688 ne saurait donc être invoquée par les États-Unis - et ses alliés - pour justifier les attaques perpétrées contre l'Irak les 3 et 4 septembre 1996 puisqu'elle n’interdit pas la présence militaire irakienne dans le Kurdistan, ne limite pas la souveraineté de l'Irak sur cette partie de son territoire national et ne contient aucune autorisation de recourir à la force soit pour acheminer laide humanitaire, soit pour protéger les droits des Kurdes. ${ }^{278}$ Cette action armée n’a pas été conduite sur le fondement juridique d'une décision du Conseil de sécurité, seul organe habilité par la Charte à user de la contrainte dans les relations internationales. Dans ce cas, il est aisé d'affirmer que le

274 DJIENA WEMBOU (Michel-Cyr), Le droit international dans un monde en mutation, essais écrits au fil des ans, L'Harmattan, Paris, 2003, p.105.

275 Ibid.

276 Ibid.

277 Ibid, p.127.

$278 \quad$ Ibid, p.127. 
système de démocratie représentative incarné par le Conseil de sécurité est fortement remis en cause par l'action unilatérale de certains groupes d'États membres de l'ONU. ${ }^{279}$

Face à ce constat, nous-nous interrogeons si le Conseil de paix et de sécurité du l'UA ne sera pas confronté aux mêmes difficultés que le Conseil de sécurité des Nations Unies? Cette question est pertinente et légitime dans la mesure où cet organe restreint a été construit sur le même modèle et doté des mêmes attributions que le Conseil de sécurité des Nations Unies. Il n’est pas aisé de répondre à cette préoccupation car l'UA est une organisation très jeune qui hérite d'une situation politico-économique générale difficile sur le continent. Toutefois, en évitant de tomber dans un optimisme béat, nous osons bien croire qu’en raison de la solidarité régionale qui caractérise les organisations régionales comme l'UA, les États membres mettront tout en œuvre afin que le Conseil de paix et de sécurité puisse pleinement assumer ses responsabilités destinées à garantir une sécurité collective indispensable au continent.

En dépit de ses insuffisances, le système de démocratie représentative institué dans les organisations internationales par le truchement des organes restreints a un effet considérable sur le fonctionnement des organisations internationales. Elle montre la complémentarité qui existe entre les organes pléniers et les organes restreints.

\section{SECTION II : L'INDISPENSABLE COMPLEMENTARITE POLITIQUE ENTRE LES ORGANES PLENIERS ET LES ORGANES RESTREINTS}

Au préalable, une précision s'impose. Nous entendons par le terme politique, les finalités voulues et poursuivies par les États membres à travers les différents organes institués au sein des organisations internationales.

Au regard des actes constitutifs, il est donné de constater que dans la mise en œuvre des objectifs politiques des organisations internationales, il existe une véritable complémentarité entre les organes pléniers et les organes restreints. Nous-nous efforcerons à mettre en évidence les manifestations (paragraphe I) et les limites de cette complémentarité (paragraphe II).

279 La solution que nous proposons face à l’unilatéralisme de certains États au Conseil de sécurité est double. D’abord, il est important de rester fidèle aux mécanismes de la Charte de l'ONU qui considère que le recours à la force n'est légal que s'il est autorisé uniquement par le Conseil de sécurité. Le Conseil de sécurité étant l'organe principal chargé d'agir lorsqu'il y a des situations de crises politiques, tout recours à la force ne doit se faire sans son aval. Ensuite, dans une situation de crise urgente l'Assemblée générale doit absolument être associée à la prise de décision. En plus d'une autorisation du Conseil de sécurité, il faut que l’Assemblée générale, à travers une session extraordinaire, se prononce sur la question du recours à la force. Laisser au Conseil de sécurité le pouvoir exclusif de prendre la décision du recours à la force peut s'avérer inefficace en raison du nombre très réduit de ses membres qui ne reflètent pas forcément la volonté générale de l’ensemble des membres de l’organisation.Une décision de recours à la force doit passer par un vote du Conseil de sécurité et de l'Assemblée générale réunie en session extraordinaire d’urgence. 


\section{PARAGRAPHE I : LES MANIFESTATIONS DE LA COMPLEMENTARITE}

La complémentarité existant entre les organes pléniers et les organes restreints se perçoit à travers le statut de chacun de ces organes. Dans les organisations internationales, il faut nécessairement des organes qui délibèrent sur la politique visée par les États membres et des organes qui agissent concrètement en vue de matérialiser dans la réalité cette politique. Comme le souligne à juste titre le Professeur DUPUY « délibérer est le fait de plusieurs, agir celui du petit nombre. ${ }^{280}$ Dans les faits, les organes pléniers correspondent au mieux aux organes délibérants des organisations internationales(A). En revanche, les organes restreints ont les atouts nécessaires pour assumer le statut d'organes exécutants dans les organisations internationales (B).

\section{A : LE STATUT D’ORGANES DELIBERANTS DES ORGANES PLENIERS.}

Il est nécessaire de définir la notion d’organes délibérants des organisations internationales. En effet, dans chaque organisation, il y a un organe dans lequel peuvent être présents tous les membres. ${ }^{281}$ Dans sa nature, il s'agit d’un organe plénier où tous les membres ont la possibilité de s'informer et d'exprimer leur point de vue sur les activités de l'organisation. ${ }^{282}$

C'est cet organe de type plénier qui est chargé de définir l'orientation de l'organisation, d'approuver son budget et sa gestion financière et, en collaboration normalement avec d'autres organes, de décider de l'admission, la suspension et l'exclusion des membres. ${ }^{283}$ Dans le cadre de l'ONU, l’article 9 de la Charte dispose que « l’Assemblée générale se compose de tous les membres des Nations Unies.

Chaque membre a cinq représentants au plus à l'Assemblée générale. $»^{284}$

Etant un organe ouvert à l'ensemble des membres de l'organisation, il est plus apte à débattre des problèmes touchant aux intérêts des États. Il apparaît que le caractère d'organe délibérant de l'Assemblée générale de l'ONU résulte de son ouverture à tous les membres et aussi de capacité à définir les grandes orientations politiques de lorganisation. Ses sessions annuelles sont perçues comme un forum d'échanges politiques par les États membres.

L’Assemblée générale délibère sur les questions politiques fondamentales pour l’organisation. A défaut de les énumérer toutes, les plus significatives sont les suivantes :

- Etudier les principes généraux de coopération pour le maintien de la paix et la sécurité internationales. ${ }^{285}$

280 DUPUY (René-Jean), Le droit international, Que sais-je ?, n 1060, op.cit., p.82.

281 DIEZ DE VELASCO VALLEJO (Manuel), Les organisations internationales, op.cit., p.78.

282 Ibid.

283 Ibid.

284 Voir l'article 9 de la Charte des Nations Unies.

285 Voir l'article 11 de la Charte des Nations Unies. 
- Examiner et approuver le budget de l'organisation. ${ }^{286}$

- Elire ou nommer les membres non permanents du Conseil de sécurité, ${ }^{287}$ tous les membres du Conseil économique et social, ${ }^{288}$ les juges de la $\mathrm{CI}{ }^{289}$ et le Secrétaire général ${ }^{290} \ldots$

L'Assemblée générale de l'ONU assume pleinement son statut d’organe délibérant au sein des Nations Unies. Il en va de même pour l'Assemblée de l'Autorité des fonds marins. Aux termes de l'article 160 de la Convention de Montégo Bay, «...l’Assemblée a le pouvoir d'arrêter, en conformité avec les dispositions pertinentes de la Convention, la politique générale de l'Autorité sur toute question ou tout sujet relevant de la compétence de celle-ci... ${ }^{291}$ En d'autres termes, l'Assemblée est l'organe qui établit les lignes générales de la politique de l'organisation. En sa qualité d’organe plénier de l'Autorité des fonds marins, l'Assemblée a le pouvoir d’énoncer des principes directeurs et de formuler des directives générales relatives au fonctionnement de l’organisation.

Les exemples peuvent être multipliés. Mais, il convient de retenir que dans les organisations internationales, il faut absolument un organe délibérant qui définit les grandes orientations politiques. Ce statut d’organe délibérant est généralement reconnu aux organes pléniers. Cependant, la présence d'un organe délibérant ne suffit pas pour assurer la mise en application de la politique générale de l'organisation. L'exitence d'un organe restreint pouvant exécuter les décisions de lorgane plénier s’avère indispensable.

\section{B : LE STATUT D’ORGANES EXECUTIFS DES ORGANES RESTREINTS}

Ainsi que le souligne le Professeur VIRALLY, « c'est la volonté, chez ses fondateurs, de disposer d'un instrument propre à la poursuite d’objectifs définis qui explique la création d'une organisation internationale.» ${ }^{292}$ La fonction instrumentale de toute organisation internationale impose la création d'un organe pouvant mettre en exécution la politique définie par les États membres.

C’est dans cette logique que sont créés les organes restreints au sein des organisations internationales. Ils ont généralement un statut d’organes exécutifs dans les organisations internationales. Le Conseil de sécurité de l'ONU jouit de compétences qui lui permettent de mettre en application le but fondamental des Nations Unies qui se résume à la recherche de la paix et de la sécurité internationales. Pour déterminer ces compétences qui permettent au Conseil d'agir

\footnotetext{
286 Voir l'article 17 de la Charte des Nations Unies.

287 Voir l'article 23 de la Charte de l'ONU.

288 Voir l'article 61 de la Charte de l'ONU.

289 Voir l'article 4 du Statut de la CIJ.

290 Voir l'article 97 de la Charte de l'ONU.

291 Voir l'article 160 de la Convention de Montégo Bay.

292 VIRALLY (Michel), Lorganisation mondiale, op.cit., p.26.
} 
efficacement au plan politique, il est nécessaire de se référer à plusieurs dispositions qui figurent aux Chapitres VI, VII, VIII et XVIII de la Charte. Nous pouvons les résumer de la façon suivante : ${ }^{293}$

- Il est habilité à enquêter sur tout différend, toute situation susceptible d'entraîner un désaccord international ou d'engendrer un différend, afin de déterminer si ceux-ci peuvent menacer la paix ou la sécurité internationale.

- Il a le pouvoir de constater l'existence de toute menace, rupture de la paix ou acte d'agression et faire des recommandations opportunes ou décider des mesures à prendre pour maintenir la paix et la sécurité internationales. Ces mesures peuvent impliquer l’emploi de la force (démonstrations, blocus et opérations exécutées par des forces aériennes, navales ou terrestres) ou ne pas l'impliquer (interruption totale ou partielle des relations commerciales, économiques et communications ferroviaires..., ainsi que la rupture des relations diplomatiques).

A l'analyse de ces dispositions, il ressort que contrairement à l'Assemblée générale qui jouit d'une compétence générale consistant à examiner la politique générale de l’organisation, le Conseil de sécurité est un organe exécutif. Il est chargé de mettre en œuvre les objectifs définis par l'ensemble des membres de l'ONU regroupé au sein de l'Assemblée générale.

Sans le Conseil de sécurité, les buts et principes des Nations Unies resteraient lettres mortes. Les membres fondateurs de l'UA se sont inspirés de ce modèle pour mettre en place le Conseil de paix et de sécurité. Cet organe restreint est ce que le Conseil de sécurité est aux Nations Unies, le bras armé qui lui est nécessaire. ${ }^{294}$ Le Conseil de paix et de sécurité peut ainsi autoriser l’organisation et le déploiement de missions d’appui à la paix, recommander à la Conférence l'intervention au nom de l'Union dans un État membre dans certaines circonstances graves (crimes de guerre, crime de génocide, crime contre l'humanité. ${ }^{295}$ Le Conseil de paix et de sécurité devient le "bras séculier " de la Conférence des Chefs d'État et de gouvernement qui a pour mission essentielle de « définir les politiques communes de l’Union. $»^{296}$

Le Conseil de l'Autorité des fonds marins est aux termes de l'article 162 de la Convention de Montégo Bay "l'organe exécutif de l'autorité. ». ${ }^{297}$ A ce titre, il contrôle l'Entreprise chargée d'explorer et d'exploiter les fonds marins, lui donne des directives, approuve les plans de travail, détermine la politique économique du système et opère le choix entre les demandeurs d’autorisation de production. ${ }^{298}$

Aux termes de larticle XII section 3 paragraphe (a) du Statut du FMI, le Conseil d’Administration est « ... responsable de la conduite générale du Fonds, et à cette fin, il exerce tous

DIEZ DE VELASCO VALLEJO (Manuel), Les organisations internationales, op.cit., p.199. MVELLE (Guy), L'Union Africaine, fondements, organes, programmes et actions, op.cit., p.183. Voir l'article 7 alinéa 1 paragraphe (e) du Protocole relatif à la création du Conseil de paix et de sécurité de l'UA. Voir l'article 9 alinéa 1 paragraphe (a) de l'Acte constitutif de l'UA.

Voir l'article 162 de la Convention de Montégo Bay. DUPUY (René-Jean), La communauté internationale, entre le mythe et l'histoire, op.cit., p.98. 
les pouvoirs que le Conseil des gouverneurs lui a délégués. ${ }^{299} \mathrm{Il}$ est l'organe chargé de mettre en œuvre la politique définie par le Conseil des gouverneurs.

De façon générale les organes pléniers tendent à s’affirmer en organes suprêmes, et à reléguer les organes restreints au rôle d'exécutants de leurs décisions, chargés des seules mesures de détails. ${ }^{300}$ Pour le bon fonctionnement des organisations internationales, une complémentarité fonctionnelle doit exister entre les organes pléniers et les organes restreints. Elle est nécessaire pour la réalisation des principes et objectifs politiques définis par l'ensemble des États membres. Mais dans la pratique, elle connait des limites.

PARAGRAPHE II : LES LIMITES DE LA COMPLEMENTARITE ENTRE ORGANES PLENIERS ET ORGANES RESTREINTS

La complémentarité qui existe entre les organes pléniers et les organes restreints des organisations internationales connaît des limites. Elles résultent d’abord du dysfonctionnement des organes restreints (A) et ensuite de leur instrumentalisation par les États membres (B).

\section{A : LE DYSFONCTIONNEMENT DES ORGANES RESTREINTS.}

Avant toute réflexion, il convient de relativiser nos analyses. En effet, le dysfonctionnement dont il s'agit ici ne concerne pas tous les organes restreints, mais seulement quelques-uns.

Cette précision faite, il est important de souligner que le mauvais fonctionnement des organes restreints peut porter atteinte à leur complémentarité avec les organes pléniers. Dans ce cas, les organes restreints seront difficilement en mesure d'exécuter la politique générale de lorganisation définie par les États membres. Plusieurs raisons peuvent valablement expliquer cette situation. Mais, nous en retiendrons deux ici : il s'agit de l'encombrement des organes restreints et du défaut de moyens matériels.

L’encombrement des organes restreints se perçoit véritablement dans le fonctionnement du Conseil de sécurité des Nations Unies. ${ }^{301}$ Cet encombrement résulte de la multiplication de la liste des États désireux de se faire entendre sans droit de vote aux discussions du Conseil. ${ }^{302}$ Cette possibilité offerte aux États non représentés est règlementée par la Charte et le Règlement intérieur provisoire du Conseil de sécurité.

Aux termes de l'article 31 de la Charte « tout État membre de l'organisation qui n’est pas membre du Conseil de sécurité peut participer, sans droit de vote, à la discussion de toute question

\footnotetext{
299 Voir l'article XII section 3, paragraphe (a) du Statut du FMI.

300 VIRALLY (Michel), Lorganisation mondiale, op.cit., p.197.

301 SMOUTS (Marie-Claude), « Réflexions sur les méthodes de travail du Conseil de sécurité », AFDI, 1982, p.610.

302 Ibid., p.603.
} 
soumise au Conseil de sécurité, chaque fois que celui-ci estime que les intérêts de ce membre sont particulièrement affectés. » ${ }^{303}$ Cette disposition est renforcée par l'article 32 qui donne aussi la possibilité à « tout membre des Nations Unies qui n’est pas membre du Conseil de sécurité ou tout État qui n’est pas membre des Nations Unies, s'il est partie à un différend examiné par le Conseil de sécurité, est convié à participer sans droit de vote, aux discussions relatives à ce différend... » ${ }^{304}$

De ces dispositions de la Charte, il convient de retenir que les États membres ou non membres directement intéressés à un conflit à lordre du jour au Conseil peuvent être invités aux débats sans droit de vote. ${ }^{305}$ Cependant, ainsi que le souligne Marie-Claude SMOUTS, « dans la pratique, le Conseil de sécurité n’a pas fait de distinction entre les États directement intéressés et les autres. Toutes les demandes de participation sans droit de vote aux travaux du Conseil sont acceptées. $»^{306}$ Cette situation a pour corollaire la multiplication des États invités à participer aux travaux du Conseil de sécurité.

Cet encombrement du Conseil de sécurité entrave sérieusement son action. ${ }^{307}$ MarieClaude SMOUTS a pu écrire à juste titre que « sous bien des aspects, le Conseil de sécurité a été gagné par la routine. La tenue, depuis quelques années, de longues sessions consacrées à la même affaire, avec audition de dizaines d'orateurs, sans qu'il y ait d'urgence particulière, ni déléments nouveaux dans la situation, a érodé sa capacité d'impulsion politique. »

Le mauvais fonctionnement du Conseil de sécurité peut entraver sa capacité à mettre en œuvre la volonté politique des États membres siégeant à l'Assemblée générale. Toutefois, cette raison seule n'explique pas les difficultés des organes restreints à jouer pleinement leur rôle d’organes exécutifs des organisations internationales. Il faut mentionner également le défaut de moyens matériels qui peut également être une cause de dysfonctionnement des organes restreints.

Pour être un organe exécutif véritablement au service de l'ensemble des membres réunis au sein des organes pléniers, il est nécessaire de doter les organes restreints de moyens matériels suffisants. A défaut de moyens matériels, ces organes ne peuvent facilement mettre en application

303 Voir l'article 31 de la Charte de l'ONU et l’article 37 du Règlement intérieur provisoire du Conseil de sécurité. Il faut préciser que l'article 37 du Règlement intérieur provisoire ne reprend pas littéralement le libellé de l'article 31 de la Charte.

304 Voir l'article 32 de la Charte de l'ONU.

305 Les invitations sont faites par le Président du Conseil de sécurité. Les « invités » en règle générale, ne prennent place à la table du Conseil qu'après l’adoption de l'ordre du jour de la séance. Aussi, les « invités » prennent la parole pour faire un exposé ou une déclaration. Cependant, les « invités » ne peuvent soulever une motion d’ordre, car ils ne peuvent s'immiscer dans la manière dont le Conseil entend conduire ses débats. Ils peuvent toutefois présenter des propositions ou des projets de résolution mais ne peuvent les mettre aux voix. SMOUTS (Marie-Claude), « Réflexions sur les méthodes de travail du Conseil de sécurité », op.cit., p.603. L'auteur précise qu' «il n’est pas rare de voir intervenir dans le débat deux fois plus d'États non membres du Conseil que d'États membres. En janvier 1982, par exemple, trente-quatre États furent invités à participer à l'examen de la situation dans les territoires arabes occupés, en avril 1982, pour examiner la question de l'Amérique centrale portée devant le Conseil par le Nicaragua, trente-et-un États furent invités, outre le Nicaragua, à participer sans droit de vote. Pendant le débat, le Conseil entendit plus de 40 orateurs. » Ibid, p.610. 
la volonté politique des États membres. L'exemple qui peut nous aider à corroborer nos propos est celui du Conseil de paix et de sécurité de l'UA. Aux termes de l'article 2 paragraphe 1 du Protocole relatif à sa création, ${ }^{308}$ le Conseil de paix et de sécurité de l’UA est « un organe de décision permanent pour la prévention, la gestion le règlement des conflits » et constitue un système d’alerte rapide, visant à permettre une réaction rapide et efficace aux situations de conflit et de crise en Afrique. »

Mais, il se pose pourtant sur le plan pragmatique, le problème de moyens. Car, il faut bien le dire, les questions de paix et de sécurité nécessitent d'importants moyens à mobiliser. ${ }^{309}$ Lanalyse de l'article 21 du Protocole révèle les difficultés de financement du Conseil de paix et de sécurité. Selon l'article 21 paragraphe 1 et 2 et 3 de cette disposition, les opérations du Conseil de paix et de sécurité seront financées par un Fonds de la paix, essentiellement alimenté par des prélèvements sur le budget de l'Union, par des contributions volontaires des États membres et par d'autres sources africaines et extra-africaines. ${ }^{310}$ Le mode de financement des opérations du Conseil de paix et de sécurité tel que prévu par ces dispositions achève de nous convaincre sur les difficultés réelles de mise en œuvre des objectifs des États membres. Au regard de la situation socio-économique de la majorité des États africains caractérisée par le sous développement, nous sommes en droit de nous interroger sur leur capacité à pouvoir alimenter le Fonds de la paix de l'Union ? En instituant le recours à d'autres sources de financements extérieures à l'Afrique, les États membres de l'UA courent le risque de faire dépendre les opérations du Conseil de paix et de sécurité envers l'aide extérieure. ${ }^{311}$ Les sources non africaines de financement chercheront sans doute à influencer les décisions du Conseil de paix et de sécurité, voire à les manipuler ou en tous cas, à couvrir des ingérences manifestes dans les affaires intérieures des pays africains. ${ }^{312}$ Des organisations internationales, des institutions et des personnalités africaines pourront apporter des concours financiers et/ ou matériels importants au Conseil de paix et de sécurité, pour lui permettre de mobiliser et de déployer des forces armées en cas de besoin. Tout le problème est de savoir si ces

308 Voir l'article 2 paragraphe 1 du Protocole relatif à la création du Conseil de paix et de sécurité de l'UA.

309 Allocution de Saïd DJINNIT, Commissaire à la paix et à la sécurité de l'UA, in BANGOURA (Dominique) et FIDIECK A BIDIAS (Émile), L'Union Africaine et les acteurs sociaux dans la gestion des crises et des conflits armés, L'Harmattan, 2006, p.24.

310 Larticle 21 paragraphe 1 est libelle comme suit : « En vue de fournir au Conseil de paix et de sécurité les ressources nécessaires pour les missions de soutien à la paix et à la sécurité, un Fonds spécial dénommé Fonds de la paix, est crée. Les opérations du Fonds sont régies par le règlement financier de l'Union. »

Quant à l'article 21 paragraphe 2, il prévoit que « le Fonds de la paix est alimenté par des crédits prélevés sur le budget ordinaire de l'Union, y compris les arriérés de contributions volontaires des États membres, et d'autres sources en Afrique, y compris le secteur privé, la société civile et les particuliers, ainsi que par des Fonds provenant d'activités de mobilisations de ressources. "

Enfin, larticle 21 paragraphe 3 souligne que « le Président de la Commission mobilise et accepte des contributions volontaires provenant de sources extérieures à l'Afrique, conformément aux objectifs et aux principes de l'Union. "

311 BIYOGUE BI NTOUGOU (Jean Delors), "Les faiblesses du système africain de prévention, gestion et de résolution des conflits ", in BANGOURA (Dominique) et FIDIECK A BIDIAS (Émile), L'Union Africaine et les acteurs sociaux dans la gestion des crises et des conflits armés, L'Harmattan, 2006, p.89.

312 DJIENA WEMBOU (Michel-Cyr), « A propos du nouveau mécanisme de l'OUA sur les conflits », in DJIENA WEMBOU, Le droit international dans un monde en mutation, essais écrits au fil des ans, op.cit., p.208. 
contributions seront effectuées régulièrement et si l'organisation peut valablement compter sur ces ressources aléatoires pour mener à bien une tâche si importante. ${ }^{313}$

Le dysfonctionnement des organes restreints résultant de leur encombrement et du défaut de moyens matériels nécessaires à leurs actions est un obstacle majeur à la complémentarité qui doit exister avec les organes pléniers. Mais, en marge de cet obstacle technique, il y a un obstacle politique qu'il convient d'analyser. Il s'agit de l'instrumentalisation des organes restreints par les États membres.

\section{B : L'INSTRUMENTALISATION DES ORGANES RESTREINTS PAR LES ÉTATS MEMBRES}

Au sens étymologique, le concept d' «instrumentalisation » vient du verbe instrumentaliser. Le dictionnaire « le Petit Larousse » définit le verbe « instrumentaliser» comme le fait « d'utiliser quelque chose à son profit. » Partant de cette définition, il faut souligner que l'instrumentalisation des organes restreints se caractérise par leur manipulation à des fins politiques par une minorité d'États membres.

En principe, les organes restreints sont créés dans le but d’exécuter la volonté générale des États membres réunis au sein des organes pléniers. Cependant, lobservation de la pratique donne de constater qu'ils sont souvent instrumentalisés par les États membres. Cette instrumentalisation des organes restreints est plus perceptible dans les organisations internationales à vocation universelle. Dans ces organisations, les rapports de force entre les grandes puissances et les petits États se traduisent généralement par une compétition pour le contrôle ${ }^{314}$ des organes restreints. Cette compétition tourne généralement à l'avantage des grandes puissances qui utilisent les organes restreints à des fins politiques. Sûrement, un exemple précis permettra de justifier cette assertion.

Lexemple du Conseil de sécurité des Nations Unies parait le plus symptomatique de la manipulation des organes restreints. La guerre déclenchée contre l'Irak le 20 mars 2003 par les États coalisés (États-Unis d'Amérique, Royaume-Uni de Grande Bretagne et d'Irlande du Nord et leurs alliés que sont la Pologne et l'Australie) ne fait pas de mystère : il s'agit d'un recours à la force armée qui constitue une violation de l'article 2 paragraphe 4 de la Charte des Nations Unies. ${ }^{315}$ Cette guerre séloigne considérablement de l'esprit de la Charte. La Charte consacre l'idée de « sécurité collective » qui a pour noyau le Conseil de sécurité. Cet organe bénéficie du monopole du recours légitime à la force armée et assume au nom de la communauté internationale, à l’exclusion de tout

\footnotetext{
313 DJIENA WEMBOU (Michel-Cyr), «A propos du nouveau mécanisme de l'OUA sur les conflits », op.cit., p.219.

314 VIRALLY (Michel), " Panorama du droit international contemporain. Cours général de droit international public », RCADI, V, Tome 183, 1983, p.262.

315 LAGHMANI (Slim), « Du droit international au droit impérial ? Réflexion sur la guerre contre l'Irak », Actualité et droit international, www.ridi./org, avril 2003, p.1.
} 
autre État ou de tout autre organe, «la responsabilité principale du maintien de la paix et de la sécurité internationales. $»^{316}$

L'instrumentalisation du Conseil de sécurité se perçoit à trois niveaux. La guerre contre l'Irak a été déclenchée par les États-Unis et ses alliés sans l'autorisation du Conseil de sécurité. L'ONU est entrée dans une nouvelle phase de turbulence avec la deuxième guerre du Golfe, déclenchée de manière unilatérale par les États-Unis, le 20 mars 2003, après avoir échoué à obtenir une autorisation du Conseil de sécurité semblable à celle donnée en 1990 par la résolution $678 .{ }^{317}$

Le Conseil de sécurité a été contraint de constater la situation de guerre engendrée par cette intervention armée. En effet, confronté à une situation juridique découlant d'une action armée non autorisée par lui, le Conseil de sécurité des Nations Unies a eu du mal à lui trouver un cadre juridique approprié. Refusant de donner une quelconque légalisation ex post facto à l'intervention, le Conseil de sécurité a dû dans un premier temps se rendre à l'évidence et qualifier, justement, la situation postérieure à l'intervention armée, en vertu de la résolution 1483 du 22 mai 2003. Dans cette résolution, le Conseil nả fait en réalité que prendre acte de la prise de contrôle de l'Irak. ${ }^{318} \mathrm{Le}$ 22 mai 2003, la diplomatie américaine a obtenu la revanche qu'elle recherchait à l'ONU. En effet, deux mois à peine après avoir déclenché la guerre sans autorisation du Conseil de sécurité, les ÉtatsUnis sont parvenus à amener le Conseil de sécurité à donner une certaine légitimité à leur guerre, puisque l'instance onusienne a été amenée à leur confier dans la résolution 1483 la reconstruction de l'Irak.

Une fois cette reconnaissance de leur occupation de l'Irak obtenue, les États-Unis, confrontés à une situation d'après guerre de plus en plus intenable, chercheront de nouveau l'aval du Conseil de sécurité. Le 16 octobre 2003, suite au vote unanime de la résolution 1511 (2003) et moyennant une petite concession accordée à l'ONU, les États-Unis sont parvenus à obtenir le feu vert de la communauté internationale pour la mise en place d'une force multinationale à laquelle les États membres de l'ONU sont appelés à fournir une assistance y compris des forces militaires. ${ }^{319}$

Les États membres de la coalition n’ont pas eu recours au Conseil de sécurité pour engager cette guerre contre l'Irak. Au contraire, le Conseil de sécurité n’a fait qu’entériner cette situation. Concernant les conséquences juridiques de ces évènements, il a été remarqué que la désuétude de la Charte des Natons Unies semble désormais très avancée et les États-Unis semblent imposer progressivement une sorte de pratique coutumière de plus en plus éloignée des principes fondamentaux du droit international. ${ }^{320}$ En effet, le non respect de larticle 2 paragraphe 2 de la Charte et l'inapplication de l'article 24 paragraphe 1 et du chapitre VII de la Charte, pour lesquels

316 BEN ACHOUR (Rafâa), "L'ONU et l'Irak I», Actualité et droit international, op.cit., p.1.

317 BEN ACHOUR (Rafâa), " L'ONU et l'Irak II », Actualité et droit international, op.cit., p.2

318 BEN ACHOUR (Rafâa), "L'ONU et l'Irak I », op.cit., p.1.

319 BEN ACHOUR (Rafâa), « La résolution 1546 du Conseil de sécurité ou l'apogée de l'art de la fiction. », Actualité et droit international, op.cit., p.1.

320 FERRAJOLO (Ornella), « La pratique et la règle de droit. Réflexions à propos de la seconde guerre du Golfe », Actualité et droit international, op.cit., p.3. 
la responsabilité principale du maintien de la paix et de la sécurité internationales appartient au Conseil de sécurité, pourraient remettre en cause non seulement le système de sécurité collective mis en place par la Charte des Nations Unies, mais encore la règle coutumière concernant le non recours à la force dans les relations entre États. ${ }^{321}$ Les États-Unis ont procédé de manière progressive afin d'imposer une sorte de pratique coutumière de plus en plus éloignée des principes fondamentaux du droit international. ${ }^{322}$

Dans ces conditions, nous pouvons dire à la suite du Professeur DJIENA WEMBOU que « l'organisation universelle apparait de plus en plus, non comme un acteur autonome des relations internationales, mais comme une véritable chambre d'enregistrement de décisions prises à la maison blanche, en consultation avec certaines capitales occidentales. $»^{323}$

Il y a manifestement une instrumentalisation du Conseil de sécurité. Mais, à l'instar de cet organe restreint, les Conseils d'administration du FMI et de l'OIT subissent aussi la manipulation de certains États membres. Dans leur fonctionnement, ces organes font preuve d'une incapacité à se remettre en cause face à leurs échecs à représenter l'intérêt général en restant autonomes par rapports aux pays riches qui en sont les principaux contributeurs. ${ }^{324}$

Relativement au FMI, le Professeur Robert CHARVIN a écrit que : « Le FMI et -la Banque mondiale- sont les gardiens de l'ordre économique et financier libéral. Toute critique à l'égard de ces institutions qui font formellement partie du système des Nations Unies [...] relèverait d'un tiers mondisme attardé. » ${ }^{325}$ Et quant au Professeur Samuel Paul HUNTINGTON, il ajoute que « l'occident s'efforce et s'efforcera à l'avenir de maintenir sa position prééminente et défendre ses intérêts en les présentant comme ceux de la communauté mondiale.[...] Loccident tente, par exemple, d'intégrer les économies des sociétés non occidentales dans un système économique global qu'il domine. Il défend ses intérêts économiques par l'intermédiaire du FMI et autres institutions économiques internationales, et cherche à imposer aux autres nations les politiques économiques qu’il pense adaptées.» ${ }^{326}$

Des réflexions faites par ces auteurs, il résulte que de façon générale le FMI est perçu comme une organisation internationale au service de lordre économique des pays occidentaux. Ce constat a absolument une influence sur son organe restreint qui est le Conseil d'administration. Comme le souligne encore le Professeur Robert CHARVIN, « il est cependant difficile de voir dans le FMI autre chose qu'une institution au service, non de lordre international, mais d'un

\footnotetext{
321 Ibid.

322 CHARVIN (Robert), "La guerre Anglo-América ine contre l'Irak et le droit international, Apocalyse law », Actualité et droit international, op.cit., p.4.

323 DJIENA WEMBOU (Michel-Cyr), Le droit international dans un monde en mutation, essais écrits au fil des ans, op.cit., p.84.

324 MVELLE (Guy), L'Union Africaine, fondements, organes, programmes et actions, op.cit., p.205.

325 CHARVIN (Robert), Relations internationales, droit et mondialisation, un monde en sens unique, Harmattan, Paris, 2000, p.67 et 68.

326 HUNTINGTON (Samuel Paul), Le choc des civilisations, Odile Jacob, Avril 2007, p.200.
} 
certain « ordre » libéral, ne serait-ce que par les modes de votation dans son instance dirigeante où les puissances industrielles ont la majorité absolue.» ${ }^{327}$ Parmi les instances dirigeantes de cette organisation internationale figure le Conseil d'administration.

L'instrumentalisation du Conseil d’administration du FMI se perçoit dans son Statut. La disposition du Statut qui fonde cette assertion est l'article XII, section 3, paragraphe (a). Aux termes de cette disposition, « le Conseil d’administration est responsable de la conduite générale du Fonds et, à cette fin, il exerce tous les pouvoirs que le Conseil des gouverneurs lui a délégués.» Quelles analyses pouvons- nous faire de cette disposition? Au niveau de l'administration de l'organisation, le Conseil d'administration ne fait que mettre en œuvre la politique définie par lorgane suprême qui est le Conseil des gouverneurs Or, en réalité le Conseil des gouverneurs reste largement dominé par les grands puissances que sont les États-Unis, la Grande-Bretagne, la France, l'Allemagne en raison de leurs contributions financières considérables au fonctionnement de l'organisation. Logiquement, la politique que le Conseil d'administration est tenue de conduire est celle voulue par ces grandes puissances. Dans les faits, comme le souligne le Professeur Robert CHARVIN, «Le FMI s’est chargé durant une première phase historique d'assurer une certaine stabilité des parités monétaires et déviter des dévaluations perturbant les échanges. Mais cette fonction s'est effacée dans les années 1970 sur l'initiative des États-Unis, suivis par les autres États occidentaux. Le FMI, prolongeant la volonté des marchés financiers, impose surtout des contraintes aux États pour imposer la fin du contrôle des changes et la dérégulation des échanges entre les places financières. $»^{328}$ La politique du FMI que le Conseil d’administration doit mettre en exécution a connu une évolution définie par les grandes puissances. D’une politique axée essentiellement vers la recherche de la stabilité monétaire et des échanges multilatéraux, le FMI s’est orientée dans le sens d'une politique favorisant lordre économique des grandes puissances. Cette instrumentalisation de la politique du FMI s'est fortement ressentie dans ses rapports avec les États du tiers monde. A ce sujet, le Professeur Robert CHARVIN révèle encore que « le FMI intervient aussi pour imposer aux États du Sud l'ouverture de leur marché et l'orientation de leurs économies vers l'exportation, sauf à renoncer à toute possibilité d’emprunt sur les marchés financiers. Les plans d'ajustement structurel établis sans grande considération pour les spécificités des pays concernés, sans préoccupation pour la situation sociale visent à éviter toute crise de la dette. $»^{329}$

La vocation des organes restreints à être des compléments indispensables aux organes pléniers dans le fonctionnement des organisations internationales semble être remis en cause d'une part par leur dysfonctionnement et d'autre part par leur instrumentalisation par les États membres. Toutefois, il faut reconnaitre que les organes restreints assument assez pleinement le rôle

CHARVIN (Robert), Relations internationales, droit et mondialisation, un monde en sens unique, op.cit., p.68.

CHARVIN (Robert), Relations internationales, droit et mondialisation, un monde en sens unique, op.cit., p.68.

Ibid., p.68. 
fonctionnel pour lequel ils ont été institués dans les organisations internationales. Dans l'ensemble, ils permettent aux organisations internationales de répondre avec célérité aux besoins des États membres. Cependant, cette contribution fonctionnelle népuise pas les raisons de la mise en place des organes restreints dans les organisations internationales. Les États membres, particulièrement les grandes puissances ont aussi une autre motivation en créant des organes restreints. Il s'agit de la quête de l'hégémonie dans les organisations internationales. 
TITRE II : LA PROBLEMATIQUE DE L'HEGEMONIE DES ÉTATS MEMBRES DANS LES ORGANES RESTREINTS

L’« hégémonie des États » s'entend de la domination et la situation privilégiée de certains États au sein des organes restreints. Cette domination qui traduit l'inégalité des États pose des problèmes au regard des règles de fonctionnement des organisations internationales. La technique des organes restreints permet, sans nécessairement porter atteinte à légalité juridique entre les membres, d'assurer une double représentation à certains d'entre eux, de renforcer leur influence au sein de l'organisation en tenant compte de la distribution réelle de la puissance. ${ }^{330}$

Contrairement aux organes pléniers qui sont marqués par une égalité juridique entre les États membres, les organes restreints expriment considérablement les rapports de force entre ceuxci. Il s'ensuit une quête permanente de l'hégémonie des États membres au sein de ceux-ci. Dans les faits, cette volonté de suprématie se perçoit à travers l'émergence d'une oligarchie d'États dans les organes restreints (Chapitre I) Mais, il est important de souligner que cette oligarchie d'États fait lobjet de contestations (Chapitre II). 


\section{CHAPITRE I : L'EMERGENCE DES OLIGARCHIES D'ÉTATS DANS LES ORGANES RESTREINTS}

Une oligarchie est un régime politique dans lequel l’autorité est entre les mains de quelques personnes ou de quelques familles puissantes. En d'autres termes, il s'agit du pouvoir de domination politique d'un petit groupe d'individus sur la majorité. Dans le cadre des relations internationales, en effet, l'histoire de la société internationale nous enseigne qu'il a toujours existé une oligarchie interétatique qui sarrogeait la direction des affaires mondiales. ${ }^{331}$ Cette oligarchie d'États apparaît dans les organes restreints des organisations internationales. Pour le justifier, nous montrerons les conditions de leur formation (Section I) et la consécration des inégalités dans les organes restreints (Section II). 
Afin de cerner les contours de la notion d'oligarchie d'États dans les organes restreints, il est important d'analyser leurs critères de formation (Paragraphe I) et leurs effets dans les organisations internationales (Paragraphe II).

\section{PARAGRAPHE I : LES CRITERES DE FORMATION DES OLIGARCHIES D'ÉTATS DANS LES} ORGANES RESTREINTS

Les oligarchies d'États dans les organes restreints se forment généralement à partir de deux critères. Nous avons d'une part celui de la contribution aux activités de lorganisation (A), et la répartition géographique équitable d’autre part (B).

\section{A : LA CONTRIBUTION AUX ACTIVITES DE L'ORGANISATION}

La contribution aux activités de l'organisation est l'un des critères qui président le choix des membres des organes restreints. Dans sa mise œuvre, ce critère prend en considération l'apport matériel des États membres au fonctionnement des organisations internationales.

Les organisations internationales déploient une diversité d’activités sur la scène internationale. ${ }^{332}$ Ces activités nécessitent pour leur mise en œuvre, des moyens matériels et humains. Or en réalité, la majorité des organisations internationales, à l'exception de quelques unes (FMI, Banque mondiale...), ne dispose pas de ressources propres. Ce sont les contributions (obligatoires ou volontaires) et autres modes de financement (la retenue opérée sur les traitements de leurs fonctionnaires et agents, les recettes dégagées par la vente de livres, revues et documents, la vente de timbres, la rémunération des services fournis dans certaines situations, les dons privées) qui permettent aux organisations de fonctionner et d'accomplir leurs missions. ${ }^{333}$

La contribution des États au fonctionnement régulier des organisations internationales revêt une importance capitale. Dans la répartition des sièges dans les organes restreints, elle constitue un critère fondamental. La Charte de l'ONU, précisément à travers l'article 23 mentionne formellement ce critère dans la désignation des membres du Conseil de sécurité. Cette disposition est ainsi libellée : «le Conseil de sécurité se compose de quinze membres de l'organisation. La République de Chine, la France, la Russie, le Royaume-Uni de Grande-Bretagne et d'Irlande du Nord et les États-Unis d'Amérique sont membres permanents du Conseil de sécurité. Dix autres membres de lorganisation sont élus, à titre de membres non permanents du Conseil de sécurité, par l’Assemblée générale qui tient spécialement compte en premier lieu, de la contribution des

\footnotetext{
332 Voir supra.

333 YAO-N'DRE (Paul), Droit des organisations internationales, op. cit., p.74.
} 
membres de l'organisation au maintien de la paix et de la sécurité internationales et aux autres fins de l'organisation...»

Cette disposition de la Charte révèle que le critère de la contribution aux activités de lorganisation est le critère principal qui fonde la composition du Conseil de sécurité. De ce fait, il justifie le choix des cinq membres non élus. Ceux-ci figurent parmi les plus importants contributeurs au budget de l'organisation. ${ }^{334}$ Lors de lélection par l'Assemblée générale des dix membres non permanents, ce critère est rigoureusement appliqué. Ce qui signifie que les États candidats à l'élection à un siège au Conseil de sécurité, doivent au préalable satisfaire aux exigences du critère « de la contribution au maintien de la paix et de la sécurité internationales et aux autres fins de l'organisation.» Cette condition concerne la taille des États, qui est en relation directe avec la contribution qu'ils peuvent fournir. ${ }^{335}$ Elle a été introduite à la Conférence de San Francisco sur l'insistance d'un certain nombre d'États qui souhaitaient assurer au Conseil de sécurité la représentation des puissances moyennes à côté des membres permanents. ${ }^{336}$

Dans la même logique, le Conseil de paix et de sécurité de l'UA tient compte dans sa composition du critère de la contribution aux activités de l'organisation. Mais, contrairement au Conseil de sécurité des Nations Unies, il n’en fait pas un critère principal. Ce constat découle de l'article 5 paragraphe 2 du Protocole relatif à la création du Conseil de paix et de sécurité qui dispose qu' " en élisant les membres permanents du Conseil de paix et de sécurité, la Conférence applique le principe de la représentation régionale équitable et de la rotation, et tient compte des critères ciaprès pour chaque État membre postulant :

d) La contribution à la promotion et au maintien de la paix et de la sécurité en Afrique ;

g) la contribution au Fonds de la paix et/ ou à un Fonds spécial créé pour un but spécifique ;

i) l'engagement à honorer les obligations financières vis-à-vis de l'Union.

Nous pouvons aussi mentionner le Conseil d'administration du FMI parmi les organes restreints qui appliquent le critère de la contribution aux activités de l'organisation. Aux termes de la Section 3 alinéa (b) du Statut du FMI, il ressort que « le Conseil d'administration est composé d’administrateurs et présidé par le Directeur général. Les administrateurs sont choisis comme suit :

i) cinq sont nommés par les États membres disposant des quotes-parts les plus élevés ;

ii) quinze sont élus par les autres États membres. $»^{337}$

Le Statut du FMI tient compte du critère de la contribution aux activités de l'organisation dans la composition du Conseil d'administration. Dans cet organe restreint, les États membres ayant les quotes-parts les plus élevés ont le droit de nommer directement cinq administrateurs. Ces États sont les États-Unis (17,09\% des quotes-parts) le Japon (6,13\% des quotes-parts), l’Allemagne

Voir à ce sujet le tableau I figurant dans les annexes. 
(5,99\% des quotes-parts), la France (4,94\% des quotes-parts) et la Grande-Bretagne (4,94\% des quotes-parts).

Le critère de la contribution aux activités de l'organisation est aussi mentionné dans la composition du Conseil d'administration de l'Autorité des fonds marins. Aux termes de l'article 161 de la Convention de Montégo bay de 1982, « le Conseil se compose de 36 membres de l'Autorité, élus par l’Assemblée dans l'ordre suivant :

a) quatre membres choisis parmi les États parties dont la consommation ou les importations nettes de produits de base relevant des catégories des minéraux devant être extraits de la zone ont dépassé, au cours des cinq dernières années pour lesquelles il existe des statistiques, $2 \%$ du total mondial de la consommation ou des importations de ces produits de base $[\ldots]$, ainsi que le plus grand consommateur ;

b) quatre membres choisis parmi les huit États parties qui ont effectué directement ou par l'intermédiaire de leurs ressortissants, les investissements les plus importants pour la préparation et la réalisation d'activités menées dans la zone...;

c) quatre membres choisis parmi les États parties qui, sur la base de la production provenant des zones soumises à leur juridiction, sont parmi les principaux exportateurs nets des catégories de minéraux devant être extraits de la zone... $»^{338}$

La Convention de Montégo Bay a pris en considération dans la répartition des sièges au Conseil le poids économique de certains États membres. Il s’agit des États qui consomment le plus les produits extraits des fonds marins, des États qui investissent considérablement dans la préparation et la réalisation des activités menées dans les fonds marins et des États qui exportent les produits des fonds marins. L’idée de prendre en considération les intérêts économiques des États dans la composition du Conseil émane des pays développés. Du point de vue des pays industrialisés, et notamment ceux qui avaient un intérêt direct à l'exploitation des ressources de la Zone, un Conseil composé uniquement sur la base du principe de la répartition géographique équitable se révélait inacceptable, car il les aurait placé dans une situation d'infériorité numérique semblable à celle qu'ils connaissaient à l'Assemblée. ${ }^{339}$ C'est pourquoi ils insistèrent sur une représentation des intérêts. ${ }^{340}$ Le principe de la représentation des intérêts a, en fin de compte, été accepté par tous les Etats à la Conférence, même si pendant un certain temps, il a subsisté des divergences dopinion quant au nombre de membres qui devait représenter les intérêts spéciaux. Finalement, on en est arrivé à une formule de compromis dont la complexité révèle les difficultés des négociations. ${ }^{341}$ D’après cette formule, le Conseil se composera de 36 membres élus par l'Assemblée, dont la moitié sera élue selon le critère de la représentation des intérêts. ${ }^{342}$

338 Voir l'article 161 de la Convention de Montégo Bay.

339 DUPUY (René-Jean), VIGNES (Daniel), Traité du nouveau droit de la mer, op.cit., p.636.

$340 \quad$ Ibid.

341 Ibid.

342 Voir l'article 161, paragraphe 1, lettre a, b, c, d, de la Convention de Montégo Bay de 1982 sur le droit de la mer. 
Larticle 7 de la Constitution de l'OIT relative à la composition du Conseil d'administration fait aussi référence au critère de la contribution aux activités de l'organisation. Aux termes de cette disposition, «1.le Conseil d’administration sera composé de cinquante-six personnes :

Vingt-huit représentent les gouvernements,

Quatorze représentent les employeurs, et

Quatorze représentent les travailleurs.

2. Sur les Vingt-huit personnes représentant les gouvernements, dix seront nommés par les membres dont l'importance industrielle est la plus considérable et dix-huit seront nommés par les membres désignés à cet effet par les délégués gouvernementaux à la Conférence, exclusion faite des délégués des dix membres susmentionnés.... » ${ }^{343}$

Cette disposition de la Constitution de l'OIT résume à elle seule l'importance qui est accordée à la contribution des États membres dans cette organisation. Les dix États « dont l'importance industrielle est la plus considérable » jouissent d’un privilège non négligeable. Sur les vingt-huit sièges disponibles au Conseil d'administration, dix sièges leur sont réservés d'avance. Ces États, à l'issue des élections du 6 juin 2005 sont : les États-Unis, la France, la Fédération de Russie, le Japon, le Brésil, l'Inde, l'Italie, la Chine, le Royaume- uni, l’Allemagne.

La contribution des États est indispensable au fonctionnement des organisations internationales. Elle a été instituée comme critère majeur dans la composition des organes restreints. Mais, la contribution des États ne doit pas être limitée au plan financier. Les États peuvent contribuer au fonctionnement de lorganisation par d'autres moyens. Dans le cadre des interventions militaires de l'ONU, les États africains ont pu contribuer à la recherche de la paix en mettant leurs soldats à la disposition de l'organisation. ${ }^{344}$ Cette contribution se réalise à travers les opérations de maintien de maintien de la paix. Le maintien de la paix peut se définir comme un:

"Field operations established by the United Nations, with consent of the parties concerned, to help control and resolve conflicts between them, under United Nations command and control, at the expense collectively of the member states, and with military and other personnel and equipment provided voluntary by them, acting impartially between the parties and using force

343 Voir l'article 7 de la Constitution de l'OIT.

344 Selon Monsieur WULLSON MVOMO Ela, «s'il faut trouver un sens à la participation de l'Afrique à l'effort de paix, l'on peut dire que celle-ci est à la fois politique et économique. Politique, parce qu'il y va de sa survie. En effet, l'Afrique ne peut se permettre, sans compromettre durablement son développement, d'être à la fois le centre de la géopolitique mondiale des conflits et de la pauvreté. Au surplus, nul ne peut dédaigner le rayonnement que procure toute politique de projection. De ce point de vue, les missions de l'ONU offrent aux pays africains l'opportunité de participer concrètement à la structuration de l'ordre stratégique mondiale. Et pour ce qui est des bénéfices économiques de cette participation, il faut savoir que la rétribution des participants est importante pour des armées connues pour la précarité de leurs ressources financières et budgétaires. » (Voir WULLSON MVOMO Ela, «L'Afrique à l'ONU : le pari de la paix et de la sécurité à lère bipolaire et post-bipolaire. Plaidoyer pour le multilatéralisme », in L'ONU vue d'Afrique, op.cit., p.128. 
minimum extent necessary. $»^{345} \mathrm{Il}$ resulte de cette définition que le maintien de la paix contient trois principes majeurs : le consentement des parties, l'impartialité des troupes de l'ONU et le non usage de la force sauf en cas de légitime défense.

Des statistiques souvent mal connues du grand public indiquent que la participation de l'Afrique à l'effort de paix est importante. Et les tableaux ci-après en rendent compte. ${ }^{346}$

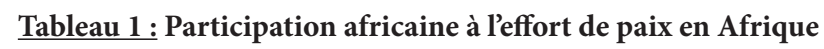

\begin{tabular}{|c|c|c|c|c|}
\hline MISSION - PAYS - DUREE & EFFECTIFS & $\begin{array}{l}\text { NOMBRE PAYS } \\
\text { AFRICAINS/ } \\
\text { TOTAL } \\
\end{array}$ & $\begin{array}{l}\text { PERTES } \\
\text { (TUES) }\end{array}$ & $\begin{array}{c}\text { BUDGETS } \\
\text { (MILLIONS \$) }\end{array}$ \\
\hline $\begin{array}{l}\text { 1) ONUC - Congo } \\
\text { Juillet } 60 \text { - Juillet }\end{array}$ & 19828 militaires & $10 / 30$ & 250 & 400.1 \\
\hline $\begin{array}{l}\text { 2) GANUPT Namibie - Angola } \\
\text { Avril } 89 \text { - Mars } 90\end{array}$ & $\begin{array}{l}7993 \text { militaires, } \\
\text { policiers et civils }\end{array}$ & $7 / 50$ & 19 & 368.6 \\
\hline $\begin{array}{l}\text { 3) UNAVEM I Angola } \\
\text { Janvier } 89 \text { - Avril } 91 \\
\end{array}$ & $\begin{array}{c}70 \text { militaires } \\
\text { (observateurs) }\end{array}$ & $2 / 10$ & Aucune & 16.4 \\
\hline $\begin{array}{l}\text { 4) UNAVEM II Angola } \\
\text { Mai } 91 \text { - Juin } 97\end{array}$ & $\begin{array}{l}1118 \text { militaires, } \\
\text { policiers et civils }\end{array}$ & $8 / 24$ & 5 & 175.8 \\
\hline $\begin{array}{l}\text { 5) UNAVEM III Angola } \\
\text { Février } 95 \text { - Juin } 97 \\
\end{array}$ & $\begin{array}{l}8405 \text { militaires, } \\
\text { policiers et civils }\end{array}$ & $11 / 38$ & 33 & 672.1 \\
\hline $\begin{array}{l}\text { 6) MONUA Angola } \\
\text { Juillet } 97\end{array}$ & $\begin{array}{l}1213 \text { militaires, } \\
\text { policiers et civils }\end{array}$ & $12 / 35$ & 12 & 140.8 \\
\hline $\begin{array}{l}\text { 7) MINURSO Sahara occidental } \\
\text { Avril } 91\end{array}$ & $\begin{array}{l}397 \text { militaires, } \\
\text { policiers et civils }\end{array}$ & $3 / 27$ & 8 & 65.1 \\
\hline $\begin{array}{l}\text { 8) ONUSOM I Somalie } \\
\text { Avril } 92 \text { - Mars } 93\end{array}$ & $\begin{array}{l}947 \text { militaires et } \\
\text { civils }\end{array}$ & $3 / 16$ & 8 & 42.9 \\
\hline $\begin{array}{l}\text { 9) ONUSOM II Somalie } \\
\text { Mars } 93 \text { - Mars } 95 \\
\end{array}$ & $\begin{array}{c}28000 \text { soldats et } \\
\text { policiers }\end{array}$ & $6 / 34$ & 148 & 1600 \\
\hline $\begin{array}{l}\text { 10) ONUMOZ Mozambique } \\
\text { Décembre } 92 \text { - Décembre } 94\end{array}$ & $\begin{array}{l}9424 \text { militaires, } \\
\text { policiers et civils }\end{array}$ & $7 / 40$ & 24 & 486.7 \\
\hline $\begin{array}{l}\text { 11) MONUOR Ouganda - } \\
\text { Rwanda } \\
\text { Juin } 93 \text { - Septembre } 94\end{array}$ & $\begin{array}{l}81 \text { militaires et } \\
\text { civils }\end{array}$ & $3 / 9$ & Aucune & 2.3 \\
\hline $\begin{array}{l}\text { 12) MINUAR Rwanda } \\
\text { Octobre } 93 \text { - Mars } 96\end{array}$ & $\begin{array}{c}5600 \text { militaires, } \\
\text { policiers et civils }\end{array}$ & $17 / 40$ & 26 & 453.9 \\
\hline $\begin{array}{l}\text { 13) MONUL Libéria } \\
\text { Septembre } 93 \text { - Septembre } 97\end{array}$ & $\begin{array}{l}368 \text { militaires et } \\
\text { civils }\end{array}$ & $4 / 22$ & Aucune & 114.2 \\
\hline $\begin{array}{l}\text { 14) GONUBA Tchad } \\
\text { Mai } 94 \text { - Juin } 94\end{array}$ & $\begin{array}{c}15 \text { militaires et } \\
\text { civils }\end{array}$ & $3 / 6$ & Aucune & 0,06747 \\
\hline $\begin{array}{l}\text { 15) MINURCA RCA } \\
\text { Avril } 98\end{array}$ & $\begin{array}{c}1379 \text { militaires, } \\
\text { policiers et civils }\end{array}$ & $9 / 12$ & 1 & 28.8 \\
\hline $\begin{array}{l}\text { 16) MONUSIL Sierra Leone } \\
\text { Juillet } 98\end{array}$ & $\begin{array}{c}90 \text { militaires, } \\
\text { policiers et civils }\end{array}$ & $2 / 10$ & Aucune & 18.3 \\
\hline
\end{tabular}

Source: Le tableau ci-dessus a été réalisé sur la base des données contenues dans 50 ans de maintien de la paix, 1948 1998, document publié par le Département de l’information des Nations unies/2004 - Mars 1999.

345 HATTO (Ronaldo), ONU et maintien de la paix : Proposition de reforme, de l'Agenda pour la paix au rapport Brahimi, L'Harmattan, Paris 2006, p.18 et 19.

346 WULLSON MVOMO (Ela), "L'Afrique à l'ONU : le pari de la paix et de la sécurité à lère bipolaire et post-bipolaire. Plaidoyer pour le multilatéralisme », in L'ONU vue d'Afrique, op.cit., p.128. 
Tableau 2: Participation africaine à l'effort de paix en Asie

\begin{tabular}{|c|c|c|c|c|}
\hline MISSION - PAYS - DUREE & EFFECTIFS & $\begin{array}{l}\text { NOMBRE PAYS } \\
\text { AFRICAINS/ } \\
\text { TOTAL } \\
\end{array}$ & $\begin{array}{l}\text { PERTES } \\
\text { (TUES) }\end{array}$ & $\begin{array}{l}\text { BUDGETS } \\
\text { (MILLIONS \$) }\end{array}$ \\
\hline $\begin{array}{l}\text { 1) UNIPOM Inde - Pakistan } \\
\text { Septembre } 65 \text { - Mars } 96\end{array}$ & $\begin{array}{l}96 \text { observateurs et } \\
\text { militaires }\end{array}$ & $1 / 19$ & Aucune & 1.7 \\
\hline $\begin{array}{l}\text { 2) UNSF Nouvelle Guinée } \\
\text { occidentale } \\
\text { Octobre } 62 \text { - Avril } 63\end{array}$ & $\begin{array}{l}1576 \text { militaires et } \\
\text { civils }\end{array}$ & $1 / 10$ & Aucune & I \\
\hline $\begin{array}{l}\text { 3) UNGOMAP Afghanistan - } \\
\text { Pakistan } \\
\text { Mai } 88 \text { - Mars } 90\end{array}$ & $\begin{array}{l}50 \text { observateurs, } \\
\text { militaires et civils }\end{array}$ & $1 / 10$ & Aucune & 14 \\
\hline $\begin{array}{l}\text { 4) MIPRENUC Cambodge } \\
\text { Octobre } 91 \text { - Mars } 90\end{array}$ & $\begin{array}{l}1090 \text { militaires et } \\
\text { civils }\end{array}$ & $4 / 24$ & Aucune & \\
\hline $\begin{array}{l}\text { 5) APRONUC Cambodge } \\
\text { Février } 92 \text { - Septembre } 93 \text { dont } \\
\text { le Cameroun }\end{array}$ & $\begin{array}{l}25795 \text { militaires, } \\
\text { policiers et civils }\end{array}$ & $10 / 45$ & 78 & 1600 \\
\hline $\begin{array}{l}\text { 6) MONUT Tadjikistan } \\
\text { Décembre } 94\end{array}$ & $\begin{array}{l}83 \text { policiers }(2) \\
\text { militaires et civils }\end{array}$ & $2 / 14$ & 6 & 22.3 \\
\hline
\end{tabular}

Source : Le tableau ci-dessus a été réalisé sur la base des données contenues dans 50 ans de maintien de la paix, 1948 1998, document publié par le Département de l’information des Nations unies/2004 - Mars 1999.

Tableau 3 : Participation africaine à l'effort de paix au Moyen-Orient

\begin{tabular}{lcccc}
\hline MISSION - PAYS - DUREE & EFFECTIFS & $\begin{array}{c}\text { NOMBRE PAYS } \\
\text { AFRICAINS/ } \\
\text { TOTAL }\end{array}$ & $\begin{array}{c}\text { PERTES } \\
\text { (TUES) }\end{array}$ & $\begin{array}{c}\text { BUDGETS } \\
\text { (MILLIONS \$) }\end{array}$ \\
\hline $\begin{array}{l}\text { 1) FUNU Sinaï - Suez } \\
\text { Octobre 73 - Juillet 79 }\end{array}$ & 6973 militaires & $2 / 13$ & 55 & 446.5 \\
\hline $\begin{array}{l}\text { 2) FINUL Liban } \\
\text { Mars 78 }\end{array}$ & $\begin{array}{c}4530 \text { militaires et } \\
\text { civils }\end{array}$ & $1 / 9$ & 227 & 136.7 \\
\hline $\begin{array}{l}\text { 3) GOMU II Iran - Irak } \\
\text { Août 88 - Février 91 }\end{array}$ & $\begin{array}{c}400 \text { militaires et } \\
\text { civils }\end{array}$ & $5 / 27$ & 177.9 \\
\hline $\begin{array}{l}\text { 4) UNYOM Yémen } \\
\text { Juillet 63 - Septembre 64 }\end{array}$ & $\begin{array}{c}189 \text { militaires et } \\
\text { civils }\end{array}$ & $1 / 11$ & Aucune & 1.8 \\
\hline $\begin{array}{l}\text { 5) MONUC Irak - Koweït } \\
\text { Avril 91 }\end{array}$ & $\begin{array}{c}1320 \text { militaires et } \\
\text { civils }\end{array}$ & $4 / 33$ & 11 & 50.6 \\
\hline
\end{tabular}

Source : Le tableau ci-dessus a été réalisé sur la base des données contenues dans 50 ans de maintien de la paix, 1948 1998, document publié par le Département de l’information des Nations unies/2004 - Mars 1999.

En plus de ce critère, il y a un autre qu'il convient d’analyser. Il s'agit de la répartition géographique équitable. 


\section{B : LA REPARTITION GEOGRAPHIQUE EQUITABLE}

Le deuxième critère qui est utilisé dans la composition des organes restreints est celui de la répartition géographique équitable. Ce critère fait reposer l'attribution des sièges sur la disposition géographique des États. ${ }^{347}$ Dans les organisations internationales contemporaines, il est élevé pratiquement au rang de principe dans les systèmes de répartition des sièges dans les organes restreints. ${ }^{348}$

Historiquement, l'acceptation de ce critère dans la formation des organes restreints des organisations internationales remonte à lépoque de la SDN. Il y fut mentionné pour la première fois le 11 septembre 1920 en ces termes : « trois membres devraient être choisis en Europe et en Amérique, le quatrième en Asie dans la ou dans les autres parties du monde. $»^{349}$

Devenu aujourd'hui incontournable, depuis l'élaboration de la Charte des Nations Unies, le principe de la répartition géographique est devenu, pour ainsi dire, une clause de style que lon retrouve dans la Charte constitutive de la plupart des organisations internationales. ${ }^{350}$ C'est donc logiquement qu'il est mentionné à l'article 23 de la Charte des Nations Unies relative à la composition du Conseil de sécurité en ces termes : « ...dix autres membres de l'organisation sont élus, à titre de membres non permanents du Conseil de sécurité, par l’Assemblée générale qui tient compte [....], d'une répartition géographique équitable. $»^{351}$ Ces dix sièges de membres non permanents sont répartis de la façon suivante :

\begin{tabular}{|c|c|}
\hline Afrique & 3 sièges \\
\hline Asie & 2 sièges \\
\hline Amérique latine & 2 sièges \\
\hline Europe occidentale et autres & 2 siège \\
\hline Europe orientale & 1 sièg \\
\hline
\end{tabular}

Mais en fait, aucun des groupes fonctionnant de façon effective ne correspond exactement à cette description purement géographique. ${ }^{352}$ Or, La concertation n’est possible quentre États

347 BENCHICK (Madjid), "Commentaire de l'article 33 de la Charte des Nations Unies », in COT (Jean-Pierre) et PELLET (Alain), La Charte des Nations Unis, commentaire article par article, $2^{\mathrm{e}}$ édition, Paris, Economica, p.439.

348 BENCHICK (Madjid), « Commentaire de l'article 33 de la Charte des Nations Unies », op.cit., p.440.

349 BOUTROS-GHALI (Boutros), " Le principe d'égalité des États et les organisations internationales » op.cit., p.48.

350 Ibid.

351 Voir l'article 23 de la Charte de l'ONU.

352 VIRALLY (Michel), Lorganisation mondiale, op.cit., p.283. 
qui non seulement se reconnaissent, mais sont liés par des intérêts communs et des conceptions communes. Il s'agit plutôt de groupes géopolitiques. ${ }^{33}$

Le Conseil économique et social des Nations Unies fait de la répartition géographique le critère essentiel du recrutement de ses membres. En application de la résolution 2847 de l’Assemblée générale, la répartition des 54 sièges du Conseil économique et social est la suivante :

- Afrique

- Asie

- Amérique latine

- Europe occidentale et autres

- Europe orientale
14 sièges
11 sièges
10 sièges
13 sièges
06 sièges.

Larticle 5 alinéa 2 du Protocole relatif à la création du Conseil de paix et de sécurité dispose qu' " en élisant les membres du Conseil de paix et de sécurité, la Conférence applique le principe de la répartition géographique équitable... $»^{354}$ En pratique, la répartition des sièges dans cet organe restreint selon les différentes zones géographique est la suivante :

$\begin{array}{ll}\text { - Afrique centrale } & 3 \text { sièges } \\ \text { - Afrique de l'est } & 3 \text { sièges } \\ \text { - Afrique du Nord } & 2 \text { sièges } \\ \text { - Afrique australe } & 3 \text { sièges } \\ \text { - Afrique de l'Ouest } & 4 \text { sièges. }\end{array}$

La Commission de l'UA ne déroge pas au critère de la répartition géographique équitable. Larticle 6 de son Statut relatif à lélection de ses membres le confirme amplement. Aux termes de cette disposition, il ressort que : «1. Lélection des membres de la commission est régie par les Règlements intérieurs de la Conférence, du Conseil exécutif, et les présents Statuts.

353 Ibid. L'auteur précise que : « le groupe latino-américain est peut-être celui qui se rapproche le plus d'une conception géographique. En fait, il s'est trouvé pendant longtemps, dans ce cas, que géographie et politique allaient ensemble [...] Nous avons aussi parlé du groupe afro-asiatique, dont l'assise géographique est bien définie. Il exclut cependant des États comme Israël, en raison de son conflit avec les États arabes. La Turquie, de son côté, bien qu'État asiatique par la plus grande partie de son territoire, est en dehors du groupe et se rattache aux États d'Europe occidentale. Le groupe des «États d'Europe orientale », est désigné habituellement par un autre vocable : celui des «États socialistes ». En effet, il regroupe en réalité les États membres s'inspirant du régime marxisme-léninisme [...] Enfin, le groupe des «États d'Europe occidentale et autres États » se présente un peu, comme son nom l'indique, comme un groupe résiduel, sans unité géographique. A côté des États d'Europe occidentale, on y trouve deux États d'Amérique du Nord (États-Unis et Canada), l'Australie et la Nouvelle-Zélande, ainsi que d'autres États du sud de l'Europe, comme le Grèce et la Turquie [...] Le point commun entre tous les membres disparates de cet ensemble est qu'il s'agit, dans tous les cas (sauf peut-être de la Turquie), d'États de population européenne (au moins par son origine), relativement développés et à économie de marché. Le critère du régime économique semble pourtant l’emporter, ce qui explique que le Japon soit maintenant pratiquement membre du «groupe occidentale». Quelques États n'appartiennent, de jure ou de facto, à aucun groupe, tels qu'Israël. » Voir le Protocole relatif à la création du Conseil de paix et de sécurité de l’UA. 
2. Les régions d’où viennent le Président et le Vice-président ont droit à un (1) Commissaire. Toutes les autres régions ont droit à deux commissaires chacun.

3. Un des membres de la Commission au moins, par région, est une femme. $»^{355}$

La lecture de cette disposition montre le souci permanent des fondateurs de l'UA d'assurer une représentation équitable à toutes les régions géographiques au sein de la Commission. La Commission étant un organe intégré, les fondateurs de l'UA sont guidés par la volonté d’assurer une représentation équilibrée et équitable des différentes personnalités que comptent les différentes régions d'Afrique.

La composition du Conseil de l'Autorité des fonds marins tient aussi compte du critère de la répartition géographique équitable. Pour s’en convaincre, il est important de se référer à l'article 161 alinéa 1 paragraphe (e) de la Convention de Montégo Bay. Il apparaît dans cette disposition en ces termes : « dix huit membres élus suivant le principe d’une répartition géographique équitable de l'ensemble des sièges du Conseil, étant entendu qu'au moins un membre par région géographique est élu en application de la présente disposition. A cette fin, les régions géographiques sont : l'Afrique, l'Amérique latine, l'Asie, l'Europe orientale (socialiste), ainsi que l'Europe occidentale et autres États. " ${ }^{356}$

Dans les organisations internationales, les critères de la contribution aux activités de l'organisation et celui de la répartition géographique équitable président au recrutement des membres des organes restreints. Mais, quelle est la portée de ces deux critères dans les organes restreints?

\section{PARAGRAPHE II : LA PORTEE DE CES DEUX CRITERES DANS LES ORGANES RESTREINTS}

Assurément, aussi bien le critère de la contribution aux activités de l'organisation que celui de la répartition géographique équitable contribuent à l'instauration des oligarchies d'États dans les organes restreints des organisations internationales. S'il est vrai que le critère de la contribution aux activités de l'organisation a une finalité aristocratique (A), force est d'admettre que celui de la répartition géographique équitable aboutit à la constitution d’une représentation sélective dans les organes restreints (B).

\footnotetext{
355 Voir l'article 6 du Statut de l'UA.

356 Voir l’article 161 alinéa 1 paragraphe (e).
} 


\section{A : LA FINALITE ARISTOCRATIQUE DU CRITERE DE LA CONTRIBUTION AUX ACTIVITES DE L'ORGANISATION}

Dire que le critère de la contribution aux activités de l'organisation vise une finalité aristocratique revient à dire que son utilisation permet de créer une caste d'États privilégiés dans les organes restreints. ${ }^{357}$ Cest dans le but de marquer la différence de poids économique entre les différents États de l'organisation que ce critère a été institué dans le recrutement des membres des organes restreints des organisations internationales. Une représentation privilégiée est accordée aux plus importants contributeurs de lorganisation internationale dans les organes restreints. Aussi, assistons-nous à la domination d'une minorité d'États développés sur la majorité de petits États dans les organes restreints.

Dans ces conditions, les États du tiers monde, particulièrement ceux d'Afrique seront toujours mis à lécart dans le processus décisionnel dans les organes restreints. A partir du poids économique réel des États africains dans les organes restreints un constat s'impose : «si l'Afrique veut être respectée au sein des organisations internationales, elle doit d'abord acquérir la puissance économique et l'influence politique suivra. Le contraire n'est pas possible, n’en déplaise aux convictions actuelles des africains». ${ }^{358}$

Aussi longtemps que le budget de fonctionnement des Nations Unies sera alloué en grande partie par les grandes puissances, on assistera toujours à la domination d'une oligarchie dans son fonctionnement. Monsieur Samir Gharbi, dans « Jeune Afrique l'intelligent » numéro 2881 du 26/09/2004 au 02/10/2004, rend compte de cet état de fait, à travers son article intitulé «Qui paie la note ? ». Il affirme : « L'ONU peine encore à ce jour à boucler son budget ordinaire : 1483 millions de dollars. Au 21 septembre 2004, 105 des 191 États membres ne sétaient pas acquittés de leur part. Les autres lui doivent encore un milliard de dollars, dont la moitié est due par les États-Unis. Les plus gros mauvais payeurs sont malins : ils n’accumulent pas plus de deux années d’arriéré et préservent ainsi leur droit de vote. Mais les plus pauvres n'y parviennent pas : 13 pays sont ainsi sous sanctions, dont 9 africains (Centrafrique, Comores, Géorgie, Guinée Bissau, Irak, Liberia, Malawi, Moldavie, Niger, Sâo-Tome et principe, Somalie, Tadjikistan et Tchad)... Le budget actuel repose à $82 \%$ sur 14 États. Certains pays pauvres n'arrivent même pas à payer les 0,001\% demandés.» ${ }^{359}$

A elle seule, cette répartition de la note du budget ordinaire des Nations Unies peut déjà inspirer une lecture froide des relations internationales. " $\mathrm{Au}$ delà des beaux discours africains, seuls ceux qui paient l'addition ont droit à la décision.» ${ }^{360} \mathrm{~A}$ partir de cette analyse, il faut comprendre que le critère de la contribution aux activités de l'organisation internationale

BOUTROS-GHALI (Boutros), «Le principe dégalité et les organisations internationales », op.cit., p.43. Il s'agit de Monsieur Kamwi John Nyambe, Ministre de l'enseignement supérieur de la Namibie, cité ici par Jean-Emmanuel PONDI, in L'ONU vue d'Afrique, Maisonneuve et Larosse / Afredit, Paris, 2003, p.333. GHARBI (Samir), cité par Jean-Emmanuel PONDI, op.cit.,p.337 et 338. PONDI (Jean-Emmanuel), L'ONU vue d'Afrique, op.cit., p.339. 
favorise irrémédiablement une " surreprésentation " des grandes puissances dans les organes restreints. Cette "surreprésentation » apparait plus clairement dans les organes restreints des organisations internationales à vocation universelle. Celles-ci, composées de tous les États de la société internationale, met en lumière la fracture économique entre les grandes puissances et les petits États. Le Conseil de sécurité des Nations Unies, le Conseil d’administration de l'OIT, le Conseil d’administration du FMI et le Conseil d’administration de l'Autorité des fonds marins traduisent parfaitement dans leur composition les clivages économiques existant entre leurs États membres. Dans ces différends organes restreints, les États représentés peuvent être classés en deux groupes : d'une part, on a les principaux bailleurs de l'organisation qui jouissent de privilèges fonctionnels exorbitants et d'autre part, les petits États qui viennent par leur présence légitimer le fonctionnement des organes restreints. ${ }^{361}$

Toutefois, un point important relatif au Conseil économique et social de l'ONU mérite dêtre relevé. Sa composition a suscité de vives controverses entre les États. Certains États estimèrent que l'Assemblée devrait assurer aux pays industriels une représentation continue. ${ }^{362}$ A l'opposé, d'autres États comme le Honduras soulignaient que le principe de légalité devait jouer ici aussi et que la représentation au sein du Conseil était très importante pour les petites nations. ${ }^{363}$ Cependant, le critère de la contribution aux activités de l’organisation n’a pas été retenu dans la mesure où une liberté d’action a été laissée à l’Assemblée générale dans le choix des membres de cet organe restreint.

Au regard de ce qui précède, il est possible de dire que le critère de la contribution aux activités de l'organisation vise essentiellement à établir l'inégalité matérielle qui existe entre les États dans les organisations internationales. En cela, il vient renforcer celui de la répartition géographique équitable qui instaure une représentation sélective dans les organes restreints.

361 A propos du rôle joué par les petits États au sein des organisations internationales, voici ce que dit Monsieur BOUTROS-GHALI : «La société internationale est une société hétérogène, composée de divers peuples. Chacun de ces peuples a sa physionomie propre qui doit être respectée, d'autant que la personnalité d'un peuple ne se mesure ni à l'étendue de son territoire, ni au chiffre de sa population, ni à la puissance de ses armements [...] La participation des petits États apporte à l'organisation internationale une contribution spécifique, car ces États sont moins directement engagés que les grandes puissances dans les conflits mondiaux et leur désintéressement , même relatif, leur permet d'exercer une action modératrice. Les petits États sont plus aptes que les grands à la collaboration internationale [...] Les petits États ne pouvant pas compter sur leur puissance matérielle pour défendre leurs intérêts, sont enclins à développer le règne du droit dans les rapports interétatiques, et il est de l'intérêt de l'organisation de soustraire progressivement les relations internationales au règne de la force pour la soumettre au règne du droit [... .] Lorganisation internationale ne peut donc se passer de la collaboration des petits États [...] En d'autres termes, il faut faire participer tous les États membres aux divers formes de l'activité collective ... » (Voir « Le principe dégalité et les organisations internationales » p.21 et 22)

362 BOUTROS-GHALI (Boutros), « Le principe d’égalité et les organisations internationales », op.cit.,p.53.

363 BAUCHARD (Denis), «Commentaire de l’article 61 de la Charte des Nations Unies », in COT (Jean-Pierre), PELLET (Alain) et FORTEAU (Mathias), La Charte des Nations Unis, commentaire article par article, $3^{\mathrm{e}}$ édition, Paris, Economica, 2005, p.942. 


\section{B : LE CARACTERE SELECTIF DU CRITERE DE LA REPRESENTATION GEOGRAPHIQUE EQUITABLE}

Le caractère sélectif du critère de la représentation géographique équitable résulte du fait qu'il favorise le recrutement d'un nombre assez limité de membres dans les organes restreints. C’est seulement certains États qui sont habilités à représenter toute une région dans les organes restreints. Dans sa mise en oeuvre, ce critère est très sélectif.

A priori, ce critère semble très avantageux pour les petits États. La simple existence physique d'un État, rattaché à un ensemble géographique, permet de revendiquer un jour un siège dans l'organe restreint de l'organisation. Ainsi, des États comme le Burundi (1970), la Biélorussie (1974), le Costa Rica (1974) ou le Koweït (1978) ont pu siéger au Conseil de sécurité des Nations Unies. ${ }^{364}$

Grâce à ce critère, n’importe quel État peut donc, à un moment donné de son histoire, participer aux travaux d'un organe restreint d'une organisation internationale. ${ }^{365}$ En outre, cette possibilité offerte aux petits États se trouve renforcée par le principe de la rotation ou encore le principe du roulement. ${ }^{366}$ Selon Monsieur BOUTROS-GHALI, " il consiste à renouveler l'Assemblée législative par moitié ou par tiers pour éviter un brusque changement et assurer une certaine continuité.

Ce critère pris isolement permet la représentation des petits États dans les organes restreints. Toutefois, c'est lorsqu'il est associé à celui de la contribution aux activités de l'organisation qu'il contribue à la formation « d’une classe de privilégiés » dans ces organes. Dans ce cas, dans sa mise en œuvre, il apparaît comme un critère sélectif. ${ }^{367}$ Généralement dans les Chartes constitutives des organisations internationales, le critère de la répartition géographique est appliqué en relation avec le critère de la contribution aux activités de l’organisation. Ainsi, l'article 23 alinéa 1 de la Charte de l'ONU le souligne en ces termes : «...Dix autres membres de l'organisation sont élus, à titre de membres non permanents du Conseil de sécurité, par l'Assemblée générale qui tient compte en premier lieu, de la contribution des membres de l'organisation au maintien de la paix et de la sécurité internationale et aux autres fins de l'organisation, et aussi d'une répartition géographique équitable... $»^{368}$

Larticle 5 alinéa 2 du Protocole relatif à la création du Conseil de paix et de sécurité de l'UA stipule que : «... En élisant les membres du Conseil de paix et de sécurité, la Conférence

BENCHIK (Madjid), « Commentaire de l'article 33 de la Charte des Nations Unies », op.cit.p.440.

Ibid, p.140.

BOUTROS-GHALI (Boutros), « Le principe d’égalité des États et les organisations internationales », op.cit.,p.41. Son caractère sélectif découle du fait que l'État candidat à un siège dans un organe restreint doit non seulement satisfaire les exigences de la contribution aux fins de lorganisation, mais aussi celles de la répartition géographique équitable.

Voir l'article 23 de la Charte des Nations Unies. 
applique le principe de la représentation régionale équitable et de la rotation, et tient compte des critères ci-après pour chaque État membre postulant :

b. la contribution à la promotion et au maintien de la paix et de la sécurité en Afrique... » ${ }^{369}$ Lanalyse de l'article 161 du Statut de l'Autorité des fonds marins montre que sa composition cherche à concilier le principe de la répartition géographique équitable avec celui de la représentation des intérêts particuliers. ${ }^{370}$ Ainsi que nous l’avons déjà souligné, le Conseil est composé de 36 membres élus par l'Assemblée qui tient compte de la répartition géographique équitable et des intérêts spéciaux des consommateurs, des importateurs, des investisseurs, des exportateurs, des États sans littoral et des États géographiquement désavantagés.

Dans sa mise en œuvre dans les organisations internationales le principe de la répartition géographique équitable est étroitement associé au critère de la contribution aux activités de lorganisation. Les États qui sont élus pour représenter les différentes régions géographiques doivent être en mesure de remplir certaines conditions tenant au fonctionnement matériel de l'organisation. Ce sont les États qui «peuvent contribuer aux fins de l'organisation » qui sont choisis pour siéger dans les organes restreints au titre des différentes régions. La mise en œuvre du critère de la répartition géographique a donc pour effet de conférer un caractère sélectif aux sièges attribués dans les organes restreints. Mais, ce caractère sélectif du principe de la répartition géographique équitable est tempéré par le principe du roulement qui entoure lélection des membres non permanents des organes restreints. ${ }^{371}$ Inspiré du droit public interne, il consiste à renouveler l'Assemblée législative par moitié ou par tiers pour éviter un brusque changement et assurer une continuité dans l'action. ${ }^{372}$ Le principe de renouvellement partiel des membres non permanents figure à l'article 23 de la Charte de l'ONU en ces termes : «Les membres non permanents du Conseil sont élus pour une période de deux ans. Toutefois, lors de la première élection des membres non permanents, trois seront élus pour une période de deux ans... $»^{373}$ Cette disposition permet à tous les États de siéger au Conseil de sécurité.

\footnotetext{
369 Voir l'article 5 du Protocole relatif à la création du Conseil de paix et de sécurité de l'UA.

370 Voir l'article 161 de la Convention de Montégo bay.

371 BOUTROS-GHALI (Boutros), "Le principe dégalité des États et les organisations internationales ", op.cit., p.41.

$372 \quad$ Ibid.

373 Voir l'article 23 de la Charte de l'ONU.
} 
En plus des nombreuses critiques dont il fait l'objet dans la doctrine, ${ }^{374}$ ce critère contribue à l'émergence d'une classe de membres privilégiés dans les organisations internationales. Mais, la notion d’oligarchie semble renforcée par la consécration des inégalités entre les États dans les organes restreints.

\section{SECTION II : LA CONSECRATION DES INEGALITES ENTRE LES ÉTATS DANS LES ORGANES RESTREINTS}

Dans les organes restreints des organisations internationales, les inégalités sont consacrées entre les États membres par les Chartes constitutives. Cette consécration résulte du fait qu’elles sont prévues et organisées par les actes constitutifs des organisations internationales. Elles s'expriment par la catégorisation des membres de ces organes (paragraphe I) par la dénaturation des conditions de vote (paragraphe II).

\section{PARAGRAPHE I : LA CATEGORISATRION DES MEMBRES DES ORGANES RESTREINTS}

Dans les organes restreints des organisations internationales, règne une véritable discrimination entre les petits États et les grandes puissances. Traduisant les rapports de force dans les relations internationales, cette catégorisation des États se manifeste par le statut de membre

374 Le principe de la répartition géographique équitable a été fortement critiqué par certains auteurs. Ainsi, le Professeur Georges Scelles soutient que « la solution continentale est certainement la moins précise et la moins recommandable. Il n’y a ni une Europe, ni une Asie, ni une Amérique...mais plusieurs. N'y a- t-il pas une Amérique latine à côté de l'Amérique anglo-saxon, et une Amérique centrale ? Pour le Professeur Alvarez, « la notion de région est approximative dans la mesure où on ne peut établir des règles pour déterminer les régions. Ce sont les circonstances et notamment les accords passés entre les États qui les composent, qui doivent montrer leur existence. » Quant à Monsieur BOUTROS-GHALI, « la mise œuvre de ce principe peut conduire à une ubiquité géographique. Pour preuve, lélection de la Turquie au Conseil de sécurité en 1951/ 1952, au siège réservé à l'Europe orientale. » (Voir à ce sujet l’article de Monsieur Boutros-Ghali sur «le principe deégalité et les organisations internationales ", déjà cité dans cette étude).

Enfin, quant au Professeur VIRALLY, il affirme que « l'expression « répartition géographique » ne va pas sans une certaine ambigüité. Fait-elle référence à la géographie physique ou à la géographie politique ? Selon qu’on envisage l'une ou l'autre, les régions n'ont plus du tout les mêmes contours. Dans le vocabulaire des Nations Unies, c'est la géographie politique qui a fini par l'emporter, en raison de l'importance grandissante prise par les "groupes ", qui possèdent normalement une assise régionale, au sens de la géographie physique, mais auxquels leurs membres appartiennent en fonction des critères politiques. Cette évolution a eu évidemment pour effet de conférer un droit de présentation, au moins officieux, au profit de chaque groupe pour les sièges revenant à «sa " région. Les dangers qui en résultent ont été dénoncés dès les premières années de l'organisation. Certains États qui, du fait de circonstances particulières, n’appartiennent à aucun groupe (Israël), ou ont été expulsés de celui auquel ils appartenaient (Cuba), ou sont dans une situation difficile en son sein, risquent en effet de se trouver exclus de toute représentation. Le danger de l'autre interprétation est qu'elle permet à des États étrangers à la région de choisir ceux qui seront appelés à la représenter. " (Voir VIRALLY (Michel), Lorganisation mondiale, op.cit., p.97). 
permanent des grandes puissances (A) et par le droit de veto dont elles jouissent dans les organes restreints (B).

\section{A : LE STATUT DE MEMBRES PERMANENTS DES GRANDES PUISSANCES}

Un aspect de la remise en cause du principe d'égalité des États dans les organes restreints des organisations internationales réside dans la différence de statut juridique entre les Etats membres. En principe, et en vertu de la règle de l'égalité absolue, l'organisation internationale ne doit pas admettre l'idée d'une participation limitée ou l'admission d'une catégorie spéciale de membres jouissant de droits restreints. ${ }^{375}$

La composition des différents organes restreints révèle l'existence de deux catégories de membres : d'une part les membres permanents et d'autre part, les membres temporaires. La première catégorie, celle des membres permanents, est constituée de membres désignés une fois pour toute par le pacte constitutif de l'organisation internationale ${ }^{376}$. Il s'agit de membres inamovibles, dont le siège n'est soumis à aucune élection.

Larticle 23 alinéa 1 de la Charte des Nations Unies ${ }^{377}$ dispose que «le Conseil de sécurité se compose de quinze membres de lorganisation. La République de Chine, la France, la Russie, le Royaume-Uni de Grande-Bretagne et d'Irlande du Nord et les États-Unis d'Amérique sont membres permanents du Conseil de sécurité. Dix autres membres de lorganisation sont élus, à titre de membres non permanents du Conseil de sécurité, par l'Assemblée générale.... ». Le Professeur Prosper WEIL décrit cette différence de statut entre les Etats membres de l'ONU en affirmant que « contrairement à l'Assemblée générale, de caractère plus representatif ou « démocratique », le Conseil de sécurité représente essentiellement l'oligarchie des cinq membres permanents, aux côtés desquels les dix autres membres ne sont guère plus, dans certaines circonstances, que des figuarants par définition transitoires et changeants $» .{ }^{378}$

Larticle 7 de la Constitution de l'OIT ${ }^{379}$ dispose que «... sur les 28 personnes représentant les gouvernements, dix seront nommées par les membres dont l'importance industrielle est la plus considérable...»

Lanalyse de l'article 161 de la Convention de Montégo Bay relative à la composition du Conseil de l'Autorité des fonds marins révèle que sur les 36 États qui composent cet organe restreint, 18 sont nommés directement par les plus gros investisseurs et les plus gros consommateurs des produits des fonds marins. En revanche, les 18 autres sont élus par la Conférence de l’Autorité des

375 BOUTROS-GHALI (Boutros), "Le principe d'égalité des États et les organisations internationales », op. cit.,p.22.

$376 \quad$ Ibid, p.39.

377 Voir l'article 23 de la Charte des Nations Unies.

378 WEIL (Prosper), "Le droit international en quête de son identité, cour général de droit international public », RCADI, 1992, VI, Tome 237, p.106. 
fonds marins ${ }^{380}$ La même approche a été suivie par l’article XII, section III du Statut du FMI. En effet, aux termes de cette disposition, « les administrateurs sont choisis comme suit :

i) cinq sont nommés par les cinq États membres disposant des quotes-parts les plus élevés.

ii) quinze sont élus par les autres États membres.»

Relativement au Conseil économique et social de l'ONU, une clarification importante s'impose. Théoriquement, au regard des dispositions de la Charte aucune différence de statut n’a été instituée par les États membres. Cependant, ainsi que le souligne BOUTROS-GHALI, « un examen attentif des quatorze élections qui ont eu lieu au Conseil économique et social, par exemple, permet de constater une régression du principe de l'égalité et la consécration d’une inégalité de fait : les cinq membres permanents du Conseil de sécurité ont été réélus de manière presqu’automatique. Quant aux treize sièges qui restaient, ils furent distribués régulièrement suivant un « virtual gentlement's agreement » fondé sur le principe de la répartition géographique équitable. ${ }^{381}$ Lélection régulière des membres permanents du Conseil de sécurité a institué une permanence de fait au Conseil économique et social.

Les exemples peuvent être multipliés. Mais, il reste bien établi que les membres des organes restreints nont pas tous les mêmes statuts juridiques. Dans ces organes, cohabitent des membres inamovibles et des membres temporaires. Cette situation est le reflet des rapports de force entre les grandes puissances et les petits États dans les organsations internationales. Elle répond au souci d'accorder aux grandes puissances certaines prérogatives fonctionnelles pour tenir compte des responsabilités accrues qui leur incombent et du pouvoir réel dont elles disposent. ${ }^{382}$

Certes, la consécration du principe dégalité des États par le droit international a marqué un progrès réel sur l’anarchie antérieur aboutissant à la prééminence des grandes puissances dans la vie internationale. ${ }^{383}$ Cependant, cette égalité doit pourtant être conçue avec réalisme. ${ }^{384}$ La représentation privilégiée accordée aux grandes puissances à travers le statut de membres permanents des organes restreints vise essentiellement à mettre en exergue l'inégalité de fait entre les grandes puissances et les petits États dans les organes restreints. En raison de leur participation considérable au fonctionnement des organisations internationales, les grandes puissances disposent des raisons assez suffisantes pour revendiquer le statut de membres permanents dans les organes restreints.

Au plan politique ce statut de membres permanents remet en cause la démocratie dans les organisations internationales. Pour BOUTROS-GHALI, «l'organisation internationale devra démocratiser l'accès aux fonctions directrices qu'exercent certains États, de par leurs aptitudes,

380 Voir l'article 161 de Convention de Montégo bay.

381 BOUTROS-GHALI (Boutros), «Le principe dégalité et les organisations internationales », op.cit., p.39.

$382 \quad$ Ibid, p.30.

383 DRAGO (Roland), "La pondération dans les organisations internationales », AFDI, 1956, p.529.

384 Ibid. 
leurs possibilités, ou leur force [...] Tous les États devront avoir la même chance de participer au fonctionnement des organes restreints et à létablissement de règles internationales. ${ }^{385}$

Le statut de membres permanents participe à la catégorisation des États dans les organisations internationales. Il en va de même pour le droit de véto qui constitue aussi un privilège considérable accordé à certains États au sein des organes restreints.

\section{B : LE DROIT DE VETO DES GRANDES PUISSANCES}

Historiquement, la notion de veto est une institution dorigine romaine. En effet, sous la République romaine, deux consuls occupent la première magistrature de l'État. Il est possible à l'un des consuls de refuser une mesure prise par son collègue. C'est ce pouvoir qu’on dénommait « le droit d'intercessio » ou droit de véto. ${ }^{386}$

Le droit de veto constitue un privilège exceptionnel et aristocratique dont disposent les grandes puissances dans les organes restreints des organisations internationales. Le concept de droit de veto en lui-même n'existe pas à proprement parler dans la Charte des Nations Unies, ${ }^{387}$ et dans d'autres actes constitutifs des organisations internationales.

Il s'agit de la faculté d’empêcher reconnue aux grandes puissances lors de l'adoption d'un texte dans les organes restreints. Plus précisément, le droit de veto est un droit qui permet aux grandes puissances d'invalider un texte en voie d'adoption lors d'un vote.

Dans le cadre de la Charte des Nations Unies, ce mécanisme est formellement reconnu aux grandes puissances à l'article 27. En effet, aux termes de cette disposition :

«1- chaque membre du Conseil de sécurité dispose d’une voix.

2- Les décisions du Conseil de sécurité sur des questions de procédure Sont prises par un vote affirmatif de neuf membres.

3- Les décisions du Conseil de sécurité sur toutes autres questions sont prises par un vote affirmatif de neuf de ses membres dans lesquels sont comprises les voix de tous les membres permanents... " ${ }^{388}$

Cette disposition selon laquelle « a security council resolution requires a super-majority of members (9-15) and, more importantly, all permanent members have a veto» ${ }^{389}$ appelle un

385 BOUTROS-GHALI (Boutros), "Le principe dégalité des États et les organisations internationales », op.cit., p.70.

386 COUlibAlY (Drissa), Le droit de véto à l'ONU, Mémoire, en vue de l'optention du Diplôme d'Etudes Approfondies (D.E.A), Droit public, l'Université Félix Houphouet Boigny d’Abidjan-Cocody, Année académique, 1996-1997, p.15.

387 ALBALA (Nuri), « Maudit droit de veto », in Le monde diplomatique, septembre 2005, p.23.

388 Voir l'article 27 de la Charte des Nations Unies.

389 GUZMAN (Andrew T.), «The consent problem in international law », httpt//ssrn.com./ abstract=1852354, p.20 
commentaire. Lalinéa 1 prévoit que pour les questions de procédure, ${ }^{390}$ la majorité requise est de neuf voix. A ce niveau, aucune différence nest établie entre les voix des membres permanents et celles des membres non permanents. En revanche, la discrimination est opérée à l'alinéa 3 «pour les questions autres que celles de procédure ». Pour ces questions de fond, il est requis toujours une majorité de neuf membres, mais comprenant absolument la voix de tous les membres permanents. Ce mécanisme implique que les États-Unis d’Amérique, la France, la Chine, la Russie, le RoyaumeUni ont chacun la possibilité de bloquer une proposition de résolution par un vote négatif quand bien même les quatre autres membres permanents et les autres membres non permanents voteraient en faveur du projet. ${ }^{391}$

Ce droit de véto des membres permanents du Conseil de sécurité sétend à des domaines très importants du fonctionnement de l'ONU. Il s'agit des questions touchant à l’admission, la suspension ou l'exclusion d'un membre. Le droit de véto en matière d'admission a pu être justifié par le fait que l'ONU ayant été créée conformément à certains idéaux, il importait de les faire respecter. C’est du moins ce qui ressort du sentiment exprimé par les délégués français et néerlandais lors de la Conférence préparatoire de San Francisco : « il est essentiel que l’admission de nouveaux membres soit régie par certaines règles afin de sauvegarder entre les membres de lorganisation, une communauté d'idéaux et de principes politiques. »" ${ }^{392}$

Dans les faits, les cinq membres permanents du Conseil de sécurité ont abondamment usé de ce privilège. En 1946, l'Afghanistan, l'Eire, l'Island, le Portugal, la Suède et la Transjordanie se déclarèrent candidats à l'admission aux Nations Unies. Les États-Unis étaient partisans de les admettre tous en bloc. Mais l'URSS mis son véto à l'admission de l'Eire, du Portugal et de la Transjordanie. En 1947, les États-Unis et la Grande-Bretagne opposèrent leur véto aux candidatures de l'Albanie et de la Mongolie.

Le droit de véto sétend aussi aux questions touchant à nomination du Secrétaire général, au maintien de la paix et de la sécurité internationales et à l'amendement de la Charte. Aux termes de l’article 97 de la Charte, le Secrétaire général «... est nommé par l’Assemblée générale sur recommandation du Conseil de sécurité... ${ }^{393}$ De ce fait, les cinq membres permanents peuvent donc opposer leur véto à une candidature au poste de Secrétaire général. Tel fut le cas du véto de la

390 Selon le Professeur Paul TAVERNIER « les questions de procédure sont celles relatives à l'inscription d'une question à l'ordre du jour, à l'ajournement de l'examen d'un point de l'ordre du jour, à la suppression d'un point de la liste des questions dont le Conseil de sécurité est saisi, à la décision du Président du Conseil de sécurité, à la suspension d'une séance, à l'ajournement d’une séance, à l'invitation à participer aux débats et à la conduite des débats. » (Voir Jean-Pierre COT et Alain PELLET, « Commentaire de l'article 27 de la Charte des Nations Unies », p.499.)

FAYE (André), «Quelle place pour l'Afrique dans un Conseil de sécurité élargi à de nouveaux membres ? , www.univ-REIMS.fr/labos CERI/ article-André FAYE- htm ou AndreFaye.metawiki.com. p.5, avril 2004, p.7.

392 COULIBALY (Drissa), Le droit de veto à l'ONU, op.cit.,p.34.

393 Voir l'article 97 de la Charte de l'ONU. 
Chine à la réélection de Kurt WALDHEIM. Sans le veto de la Chine, il aurait accédé à un troisième mandat avec le soutient actif des États-Unis, de la France, du Royaume-Uni et de l'URSS. ${ }^{394}$

Ce même système est utilisé au Conseil d’administration de l'OIT. En effet, les dix membres représentant les États dont l'importance industrielle est la plus considérable ne disposent d'aucun pouvoir de vote particulier quand le Conseil prend ses décisions. Ils bénéficient par contre, d'une prérogative spéciale quand a lieu la ratification des amendements à la Constitution de l'OIT. Conformément à l'article 36 de la Constitution de l'OIT, les amendements doivent être ratifiés par les deux tiers des membres de l'organisation, cette majorité comprenant cinq des dix membres ayant l'importance industrielle la plus considérable. ${ }^{395}$ En l'absence des voix de cinq des grandes puissances industrielles, aucune décision d'amendement ne peut être adoptée.

Il est important de mentionner un autre mécanisme similaire au droit de véto. Il s'agit de la minorité de blocage. Contrairement au droit de véto qui est attribué expressément par les actes constitutifs des organisations internationales, la minorité de blocage constitue plutôt un état de fait dans lequel la possibilité de bloquer une décision résulte d’une situation passagère -quoique souvent durable- due au nombre de voix d'un État. ${ }^{396}$ En guise d'exemple, nous pouvons relever le cas des États- Unis d'Amérique au FMI. Ce pays, qui à lui seul détient 17,14\% des quotes-parts possède une minorité de blocage au Conseil d’administration. ${ }^{397}$ Les États- Unis peuvent empêcher la prise de décision dans cet organe restreint dans la mesure où ils disposent des quotes-parts les plus importants.

\footnotetext{
394 COULIBALY (Drissa), Le droit de veto à l'ONU, op.cit., p.27.

395 BOUTROS-GHALI (Boutros), «Le principe d’égalité des États et les organisations internationales », op.cit., p. 60 et 61 .

396 KANZ (Jerzy), «Le vote pondéré dans les organisations internationales », RGDIP, 1981, p.339.

397 Les quotes-parts des principaux contributeurs du FMI, en septembre 2006, se répartissent comme suit :

- $\quad$ États-Unis : $\quad 17,14 \%$

- Japon: $\quad 6,13 \%$

- $\quad$ Allemagne : $\quad 5,99 \%$

- $\quad$ France : $\quad 4,94 \%$

- $\quad$ Grande-Bretagne : $\quad 4,94 \%$

- Chine: $\quad 3,72 \%$

- Italie : 3, $25 \%$

- Canada: $\quad 2,93 \%$

- $\quad$ Russie : $\quad 2,74 \%$

- Pays-Bas : 2, $38 \%$

- Belgique : 2, $12 \%$

- Inde : 1, $91 \%$

- Mexique : $\quad 1,45 \%$

- Espagne: $\quad 1,40 \%$

- Brésil : $\quad 1,40 \%$

- $\quad$ Corée du Sud : $\quad 1,35 \%$

- $\quad$ Afrique du Sud : $\quad 0,86 \%$

- Turquie: $\quad 0,55 \%$
}

(Source : www.ladocumentationfrançaise.fr/dossier/banque-mondiale, p.2) 
Le problème de la minorité de blocage a été posé au Conseil de l'Autorité des fonds marins par les grandes puissances. En effet, bien qu'ils se soient employés à développer dans les détails les normes régissant les fonds marins, les pays industrialisés n’ont pas négligé pour autant le système de prise de décision. ${ }^{398}$ Les pays développés ayant un intérêt spécial à l’exploitation de la Zone s'efforcèrent de faire accepter des procédures susceptibles de leur permettre de contrôler l’adoption des décisions malgré leur position minoritaire. ${ }^{399}$ Mais, cette position n’a pas été partagée par les pays du tiers monde numériquement plus importants et mieux représentés à l'Assemblée et qui soutenaient le principe selon lequel chaque État devrait avoir une voix et toutes les décisions devraient être prises à la majorité, laquelle serait de deux tiers pour les questions de fond. ${ }^{400}$ La solution qui fut finalement incorporée dans le texte de la Convention tire son origine d'une proposition du Président de la conférence, qui, dans son intention de concilier des positions aussi convergentes, n'avait d'autre issue que de diviser les questions de fond relevant de la compétence du Conseil en différentes catégories et de suggérer une majorité variable selon ces dernières. ${ }^{401}$

La reconnaissance d'un statut de membres permanents et d'un droit de véto aux grandes puissances dans les organes restreints constituent la preuve de la consécration des inégalités dans les organes restreints. Mais, elles sont accentuées par les conditions de votes inéquitables mises en place au sein de ces organes.

\section{PARAGRAPHE II : L'INSTAURATION DE CONDITIONS DE VOTES INEQUITABLES}

Les conditions de vote qui ont été instaurées dans les organes restreints sont inéquitables. Elles favorisent les grandes puissances dans les prises de décision. Cette manipulation de l’expression

\footnotetext{
398 DUPUY (René-Jean), VIGNES (Daniels), Traité du nouveau droit de la mer, op.cit., p.24.

399 Ibid, p.639.

$400 \quad$ Ibid, p.638.

401 Selon cette formule, il existe au moins cinq catégories de questions : -En premier lieu, les questions de procédure sont décidées à la majorité des membres présents et votant (article 161, paragraphe 8 , lettre a).

- En deuxième lieu, les décisions sur les questions de fond énumérées au paragraphe 8, lettre b) de l’article 161 sont prises à la majorité des deux tiers des membres présents et votant, cette majorité devant comprendre celle des membres du Conseil.

- En troisième lieu, le paragraphe 8, lettre c), du même article contient une longue énumération des questions de fond qui doivent être prises à la majorité des trois quarts des membres présents et votants, cette majorité devant comprendre celle des membres du Conseil.

- En quatrième lieu, conformément à l'article 161, paragraphe 8, lettre d), le consensus est requis : pour l'adoption de mesures en vue de la protection des États en développement contre les effets économiques défavorables visés à l'article 150, lettre h); pour la recommandation, l'adoption et l'application provisoire de règles, règlements et procédures; pour l'approbation d'amendements à la partie XI de la Convention et pour le rejet de plans de travail dont l'approbation a été recommandé par la Commission juridique et technique, mais qui font l'objet d'une objection de la part d'un membre du Conseil (article 162, paragraphe 2, lettre j, point i).

- En cinquième lieu, il existe une procédure spéciale, extrêmement complexe, pour l'approbation ou le rejet d'un plan de travail.
} 
de la volonté des membres de ces organes se réalise par la pratique de la pondération des voix (A) et par la dénaturation des règles traditionnelles de vote (B).

\section{A : LA PRATIQUE DE LA PONDERATION DES VOIX}

L'expression de la volonté de l'organisation internationale se réalise à travers le vote des différents organes. Aussi revêt-il une importance capitale dans la quête du contrôle et de la domination de celle-ci. C’est dans cette perspective que s'inscrit le vote pondéré dans les organes restreints des organisations internationales.

Le vote pondéré, selon le Professeur NGUYEN QUOC Dinh, « consiste à attribuer à chaque État membre un nombre de voix plus ou moins proportionnel à la valeur de ses apports en capital. $»^{402}$ En principe, la pondération des voix est utilisée pour traduire l'importance réelle des États dans le domaine considéré. ${ }^{403}$

Elle tient compte principalement de l'apport de chaque État membre, de telle sorte que ceux qui contribuent considérablement au financement de l'organisation puissent jouir d'un nombre de voix en adéquation avec leur participation. Il s'agit d’un ensemble de techniques selon lesquelles la compétence juridique au sein des organisations internationales est proportionnée à leurs responsabilités internationales et au rôle que chacun d'eux joue dans chaque organisation. ${ }^{404}$ La pondération vise essentiellement à donner un poids différent au vote d'un membre par rapport à celui d'un autre membre. ${ }^{405}$

Ainsi définie, la pondération des voix est beaucoup utilisée dans les organes restreints des organisations internationales économiques. Dans les institutions financières internationales, le principe « un État, une voix » est mis à lécart en faveur du vote pondéré. Au sein du Conseil d'administration du FMI, ce sont les quotes-parts de chaque État, c'est-à-dire leur souscription

\footnotetext{
402 NGUYEN QUOC (Dinh), Droit international public, op.cit., p.691.

403 DORMOY (Daniel), Droit des organisations internationales, Dalloz, 1995, p.76.

404 DRAGO (Roland), «la pondération dans les organisations internationales », AFDI, 1956, p.531.

405 MONACO (Riccardo), « Les principes régissant la structure et le fonctionnement des organisations internationales », op.cit.,p.135.
} 
au capital du Fonds, qui détermine leur droit de vote. ${ }^{406}$ Au regard donc de ce système, les pays industrialisés détiennent la plupart des droits de votes. Quant aux pays du tiers monde, réduits à une fonctionnalité marchande, ils sont marginalisés dans le système économique international incarné par le FMI. Ils n’ont pas voix au chapitre dans l'instance de décision du FMI que constitue le Conseil d'administration.

Les raisons qui militent en faveur du vote pondéré dans les organes restreints sont essentiellement d’ordre économique. Pour les pays occidentaux, la pondération se justifie pour les besoins de justice ou de bon sens. ${ }^{407}$ De ce point de vue, souligne le Professeur Jerzi KRANZ « il semble en effet justifié qu'un État membre dont la contribution financière est mille fois supérieure à celle d'un autre dispose, au moins dans certains cas, d'un plus grand nombre de voix que le second.» ${ }^{408}$ Donc, poursuit-il, «que cela nous plaise ou non, la configuration des forces au sein d'une organisation internationale économique à caractère opérationnel ne peut pas sécarter trop sensiblement du rapport des forces économiques dans le domaine règlementé.» ${ }^{409}$

Les pays occidentaux se prononcent en faveur de la pondération en raison de leur puissance économique et politique. Ils estiment que l'application du principe " un État, une voix » dans les organisations universelles à vocation économique, conduirait à l'agitation et à une majorité

406 La répartition des droits de vote des administrateurs se présentait comme suit en 2003 :

$-1^{\text {er }}$ États- Unis : $\quad(17,14 \%)$

$-2^{\mathrm{e}}$ Japon : $\quad(6,15 \%)$

$-3^{\mathrm{e}}$ Allemagne: $\quad(6,01 \%)$

$-4^{\mathrm{e}}$ France : $\quad(4,96 \%)$

$-5^{\mathrm{e}} \quad$ Royaume-Uni : $\quad(4,96 \%)$

$-6^{\mathrm{e}}$ Belgique : $\quad(5,15 \%)$

$-7^{\text {e }}$ Pays-Bas : $\quad(4,86 \%)$

$-8^{\text {e }}$ Espagne: $\quad(4,29 \%)$

$-9^{\mathrm{e}}$ Grèce : $\quad(4,19 \%)$

$-10^{\mathrm{e}}$ Canada : $\quad(3,72 \%)$

- $11^{\mathrm{e}}$ Island : $\quad(3,52 \%)$

$-12^{\mathrm{e}}$ Australie: $\quad(3,34 \%)$

$-13^{\mathrm{e}}$ Arabie Saoudite : $\quad(3,23 \%)$

$-14^{\text {e }}$ Malaisie : $\quad(3,18 \%)$

$-15^{e}$ Nigéria : $\quad(3,18 \%)$

$-16^{\mathrm{e}}$ Chine : $\quad(2,95 \%)$

- $17^{\mathrm{e}}$ Egypte : $\quad(2,95 \%)$

$-18^{\mathrm{e}}$ Suisse : $\quad(2,85 \%)$

$-19^{\text {e }}$ Russie : $\quad(2,75 \%)$

$-20^{\mathrm{e}}$ Brésil: $\quad(2,47 \%)$

$-21^{\text {e }}$ Iran : $\quad(2,47 \%)$

$-22^{\mathrm{e}}$ Inde: $\quad(2,40 \%)$

$-23^{\mathrm{e}}$ Chili : $\quad(2 \%)$

$-24^{\mathrm{e}}$ Guinée : $\quad(1,42 \%)$

Source : www.le figaro.fr/economie/, p.2

407 KRANZ (Jerzy), " Le vote pondéré dans les organisations internationales »,op.cit.,p.321.

408 Ibid.

409 Ibid. 
mécanique des pays en développement dont l'importance politique et économique n’est pas comparable à celle des pays développés. ${ }^{410}$ La pondération des voix a surtout pour but d’assurer la suprématie des grandes puissances dans la prise des décisions. Il est difficile d’imaginer la création et le bon fonctionnement du FMI sans la participation des États-Unis ou des principaux autres pays industriels. ${ }^{411}$

Dans les organisations internationales à vocation politique, la pondération des voix nest pas aussi exclue. A ce sujet, l'exemple le plus connu est le système prévu dans la Charte des Nations Unies au Conseil de sécurité. ${ }^{412}$ Le pouvoir de vote des membres du Conseil est inégal, bien que chacun d'entre eux dispose d'une voix. ${ }^{413}$ De ce fait, il y a par conséquent deux catégories de voix : d'abord les voix des privilégiés qui doivent obligatoirement faire partie de la majorité, ensuite les voix des non privilégiés qui ne sont nécessaires que dans la mesure où elles contribuent à la formation de la majorité requise. ${ }^{414}$

Dans tous les cas, la pondération des voix dans les organes restreints aboutit à la dictature des grandes puissances. ${ }^{415}$ Elle permet de rétablir entre les membres d'une organisation internationale les inégalités de puissance qui existent entre États en dehors de cette organisation, voire même de les accentuer. ${ }^{416}$ La séduction de ce mécanisme auprès des plus puissants est facile à comprendre. ${ }^{417}$ La conséquence en serait de réduire gravement les possibilités d'influence, déjà réduites, des plus faibles, qui pourraient devenir absolument négligeable. ${ }^{418}$ La pondération des voix est contraire aux principes élémentaires de la démocratie dans les organisations internationales fondée sur la règle de vote « un État, une voix ». Le principe d’égalité des États dans les organisations internationales est, en définitive lié à l'idée de démocratie internationale. ${ }^{419} \mathrm{La}$ démocratie dans les organisations internationales ne peut s'accommoder du système de la pondération des voix, qui constitue en soi une négation du principe d'égalité. Mais, la dénaturation des conditions de vote traditionnelles renforce davantage cette inégalité.

\section{B : LA DENATURATION DES REGLES TRADITIONNELLES DE VOTE}

Le droit des organisations internationales fait apparaître trois grandes règles de vote au sein des organes des organisations internationales : Il s’agit de la règle de l'unanimité, de la majorité

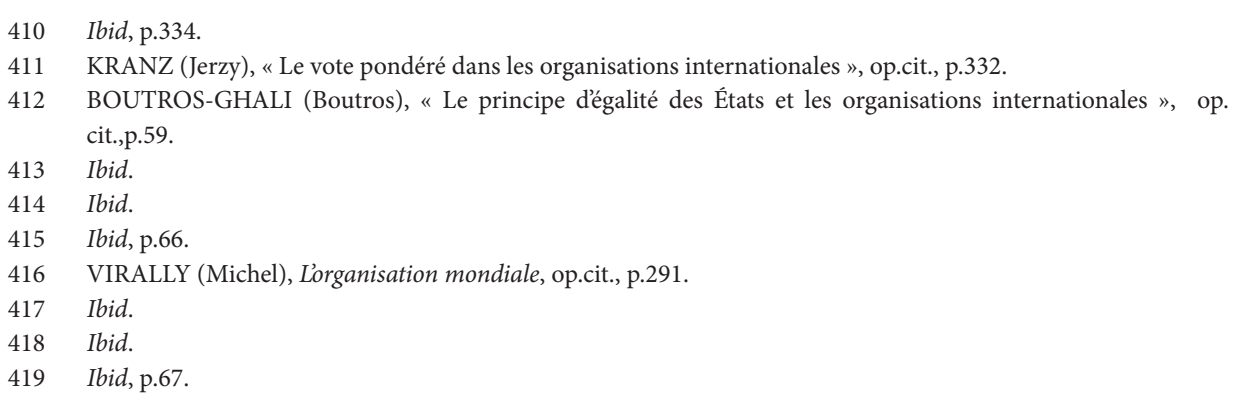


et du consensus. Le choix de l'une ou de l'autre révèle l'intensité de la solidarité collective ainsi que la mesure dans laquelle l’organisation se montre respectueuse de la liberté des États membres. ${ }^{420}$

Dans les organes restreints, les procédures de vote ont été aménagées dans le sens de la protection des intérêts des grandes puissances. Ainsi, la règle de l'unanimité plus respectueuse de l'égalité des États a été allégée dans son application par le recours à la règle de la majorité (1). Quant à la règle du consensus, elle se révèle très contraignante pour les petits États (2).

\section{(1) L’allègement de la règle de l’unanimité par celle de la majorité}

La règle de l'unanimité tire son origine historique des Conférences internationales. ${ }^{421}$ Elle signifie que la prise de décisions requiert l'accord de tous les États membres, sans exception. Elle a pour conséquence que chaque membre possède un pouvoir de vote égale à celui des autres. ${ }^{422}$

Le principe de l'unanimité ne fait aucune distinction entre les États dans le processus décisionnel. Mieux, il réalise la véritable égalité juridique entre les États. Le principe général découlant du principe de l'égalité des États est que chaque État dispose d'une voix. ${ }^{423} \mathrm{Du}$ point de vue politique, il est certainement la preuve tangible de l'expression authentique de la volonté générale. Aussi, les partisans de ce principe rappellent-ils que la démocratie est par essence placée sous le signe de l'unanimité. ${ }^{424}$ Dans ce cas, aucune majorité d'États ne saurait imposer, même à un autre État, une résolution que ce dernier n'aurait pas voté. ${ }^{425}$ Dans un organe restreint où le nombre de membres est très réduit, des votes démocratiques devraient s'exprimer par le biais du principe de l’unanimité.

Mais, ainsi que le souligne le Professeur YAO-N'DRE Paul, « le droit de vote des États dans les organisations internationales néchappe pas nécessairement à la lutte des intérêts contradictoires. » ${ }^{426}$ Face à la rigueur des effets de la règle de l'unanimité dans les organes restreints, les organisations internationales qui ont vu le jour après la deuxième guerre mondiale ont opté pour la règle de la majorité. ${ }^{427}$ Grâce à la règle « un État, une voix ", les petites puissances se sentent protégés contre l'arbitraire des grandes nations. ${ }^{428} \mathrm{~A}$ l'occasion d'un vote important à prendre dans

420 VIRALLY (Michel), Lorganisation mondiale, op.cit., p.291.

421 MONACO (Riccardo), « Les principes régissant la structure et le fonctionnement des organisations internationales »,op.cit., p.130. La Conférence internationale a un caractère temporaire et se réunit seulement en vue d'un résultat particulier que les États veulent atteindre : elle ne crée aucun système particulier qui puissent lier les États participants quant à l'expression de la volonté ; elle est aussi fondée sur le principe de légalité absolue des États et elle ne donne lieu à aucune atteinte à leur indépendance réciproque. BOUTROS-GHALI (Boutros), « Le principe d’égalité des États et les organisations internationales », op.cit.,p.27. DORMOY (Daniel), Droit des organisations internationales, op.cit., p.75

$424 \quad$ Ibid, p.29.

425 Ibid, p.27.

426 YAO-N'DRE (Paul), Droit des organisations internationales,op.cit.,p.69. 
un organe quelconque d'une institution internationale, il suffit de la coalition de petits États pour faire échec aux décisions proposées par les grandes puissances au sein des assemblées. ${ }^{429}$

Pour toutes ces raisons, les organisations internationales contemporaines, créées sous les auspices des grandes puissances ont opté pour la règle de la majorité dans leurs organes restreints. ${ }^{430}$ Au Conseil de sécurité des Nations Unies, l'article 27 de la Charte prévoit une majorité de 9 membres pour les questions de procédure. Quant aux questions de fonds, il est aussi prévu une majorité de 9 membres ; mais une majorité comprenant obligatoirement les voix des cinq membres permanents. Larticle 67 alinéa 2 relatif au Conseil économique et social dispose que "les décisions du Conseil économique et social sont prises à la majorité des membres présents et votants.»

En fait, soutient BOUTROS-GHALI, « le vote majoritaire donne la possibilité à la grande puissance de se créer une véritable clientèle. ${ }^{431}$ La clientèle de la grande puissance se constitue par le nombre d'États alliés ou partisans qui s’associent à elle afin de lui donner une position privilégiée dans le cadre du vote majoritaire. Autrement dit, ce clientélisme vise à mettre «le nombre au service de la puissance ». ${ }^{432}$ BOUTROS-GHALI souligne encore qu' " on commettrait une erreur en se représentant les États membres d'une organisation internationale comme des entités indépendantes les unes des autres. A des degrés divers, l'attitude de la plupart d’entre eux est déterminée par des liens politiques qui les unissent à certaines puissances.» ${ }^{433}$

Les procédés utilisés par les grandes puissances pour aboutir à cette clientèle sont variés. Il y a d'abord les procédés diplomatiques habituels : persuasion, pression, assistance.,etc. En échange de certains avantages, le petit État soutiendra la grande puissance dans une opération de vote. Le petit État donne pour ainsi dire une procuration générale à la grande puissance pour toutes les résolutions qui ne concernent pas ses intérêts propres. ${ }^{434}$ Ces pratiques témoignent des rapports de forces entre les États membres d'un organe restreint. La grande puissance attire dans son sillage tous les petits États lors des votes à la majorité.

Il faut ensuite mentionner les accords et les organismes régionaux - Union panaméricaine, Pacte atlantique, ensemble Afro-asiatique, bloc communiste- qui imposent à leurs membres une certaine discipline au profit des grandes puissances qui animent ces groupes ${ }^{435}$. Les accords et organismes régionaux contribuent aussi aisément à assurer la prédominance des grandes puissances par lorientation qu'ils donnent à leurs membres lors des votes majoritaires dans les organes

429 MONACO (Riccardo), «Les principes régissant la structure et le fonctionnement des organisations internationales ", op.cit., p.134.

430 Dans sa mise en œuvre, la règle de la majorité dans les organisations internationales peut être une majorité simple (la moitié des votes émis plus un) ou qualifiée (généralement les deux tiers des votes, ou une majorité encore plus importante dans laquelle interviennent représentatif de tous les groupes existants dans l'organisation).

431 BOUTROS-GHALI (Boutros), «Le principe d'égalité et les organisations internationales », op.cit., p.56.

432 VIRALLY (Michel), Lorganisation mondiale, op.cit., p.291.

433 BOUTROS-GHALI (Boutros), « Le principe d'égalité et les organisations internationales », op.cit., p.57.

434 Ibid,p. 57.

$435 \quad$ Ibid, p.58. 
restreints.Il apparaît ainsi que le système majoritaire au sein des organisations internationales offre aux grandes puissances une infinité de procédés, de "filtres ", d' " écrans ", de "freins ", pour corriger les défauts du vote égalitaire, tout en le maintenant. ${ }^{436}$

Le recours à la règle de la majorité dans les organes restreints en lieu et place de celle de l'unanimité s'avère avantageuse pour les grandes puissances. Qu’en est-il de la règle du consensus ?

(2) Le caractère contraignant de la règle du consensus pour les petits États au sein des organes restreints.

Le consensus peut s'entendre selon la pratique de l'ONU comme un accord général sans vote, mais pas nécessairement unanime. ${ }^{437} \mathrm{Il}$ traduit la volonté d’arriver à un accord découlant d'une absence d'opposition. ${ }^{438}$ Le Professeur Guy DE LACHARRIERE définit le consensus comme « une procédure de prise de décision, exclusive du vote, consistant à constater l'absence de toute objection présentée comme étant un obstacle à l'adoption de la décision en cause. » ${ }^{439}$

Politiquement, le développement de la pratique du consensus doit être rattaché à la persistance des antagonismes que connaît la société internationale. La pratique du consensus se présente comme un instrument de dialogue entre les pays développés et les pays en voie de développement. ${ }^{440}$ Un cadre véritable de discussion aboutissant à une solution de compromis s'instaure avec la pratique du consensus.

Elle trouve aujourd'hui son terrain de prédilection dans les organes des Nations Unies. ${ }^{441}$ Le Conseil de sécurité a fréquemment recours à cette procédure pour ses prises de décisions. Cette procédure est particulièrement bien adaptée à la tâche du Conseil qui est le règlement politique des conflits internationaux. ${ }^{422}$ A priori, elle permet de trouver un compromis entre les grandes puissances et les petits États qui siègent au Conseil de sécurité.

Les organes restreints des institutions spécialisées adoptent aussi des décisions par consensus. Cette pratique est courante au sein du Conseil d'administration de l'OIT depuis sa création. ${ }^{443}$ Quant aux organes restreints des organisations internationales à vocation économique, ils appliquent aussi la règle du consensus. Le Conseil d’administration du FMI utilise généralement cette procédure. Aussi, les votes formels sont-ils rares dans cet organe. ${ }^{444}$

\footnotetext{
436 Ibid, p.59.

437 CASSAN (Hervé), « Le consensus dans la pratique des Nations Unies », AFDI, 1974, p.46O.

438 DORMOY (Daniel), Droit des organisations internationales, op.cit., p.77.

439 DE LACHARRIERE ( Guy), «Le consensus en droit international », in Pouvoir , n5, p.35.

440 CASSAN (Hervé), « Le consensus dans la pratique des Nations Unies », op.cit.p.457.

$441 \quad$ Ibid,p.460.

442 Ibid, p.461. L’auteur souligne qu’entre 1946 et 1970, le Conseil de sécurité avait adopté 179 résolutions relatives au maintien de la paix et de la sécurité dont 50 avaient été par consensus.

$443 \quad$ Ibid,p.465.

$444 \quad$ Ibid, p.466.
} 
La pratique du consensus semble faire l'adhésion dans les organes restreints des organisations internationales. Elle ne pose pas de problèmes particuliers pour les grandes puissances qui l'utilisent dans leurs rapports avec les petits États. Cependant, le consensus présente des inconvénients. Le consentement d'un État sur une question ne peut s'exprimer que par un vote formel. Ce faisant, l'Etat donne sa position sur le problème en cause. Or, avec la procédure du consensus qui exclut le vote, cette liberté d’expression est enlevée aux États. Pour les petits États, la procédure du consensus est relativement contraignante car elle constitue une procédure d'approbation forcée, un moyen de provoquer artificiellement l'adhésion de tous en donnant un sens positif aux abstentions. ${ }^{445}$ Dans les relations entre les petits États et les grandes puissances, ces derniers se sont montrés méfiants à l'égard de ce processus décisionnel et, lorsqu'ils l'ont accepté par intérêt c'est plus dans la résignation que dans l'enthousiasme qu'ils l'ont fait. ${ }^{446}$

Le caractère contraignant de la procédure du consensus tient au fait qu'elle exige que des États qui nont pas les mêmes pouvoirs politiques, économiques et militaires de trouver un compromis sur des questions d'intérêts universels. Naturellement, la décision qui sera prise par consensus ne réalise "qu’un compromis sur un désaccord ". L'unanimité tacite n’est donc qu’un accord de façade. Donc, la décision prise par consensus n’est pas forcément la volonté unanime des États.

Les grandes puissances s'efforcent d'assigner à cette pratique deux rôles qu'elle ne devrait pas avoir. En orientant le consensus vers une interprétation et une pratique volontairement restrictives, les grandes puissances recherchent une nouvelle forme de véto dans le mécanisme de décision. ${ }^{477}$ En insistant sur la nécessité de la pratique permanente du consensus comme mode quasi-exclusif de prise de décision, en usant de tous les subterfuges pour cimenter cette pratique, y compris des menaces à peine voilées lors des débats et des négociations ou des retenues indument opérées sur leurs quotes-parts au budget, les grands pays entendent affirmer une nouvelle orientation du consensus qui, en dernier ressort signifierait soit la renonciation, fût-elle volontaire, soit l'utilisation marginale, de leur droit souverain de vote par les pays en développement. ${ }^{448}$

Les différents privilèges fonctionnels et les condtions de vote favorables aux grandes puissances visent à établir leur hégémonie dans les organes restreints. Toutefois, cette hégémonie fait lobjet de contestations récurrentes des petits États.

\footnotetext{
445 CASSAN (Hervé), «Le consensus dans la pratique des Nations Unies », op.cit., p.485.

446 DORMOY (Daniel), Droit des organisations internationales, op.cit., p.77.

447 MONTHE (Michel Tommo), «Quatre décennies de reformes et de renouveau au sein de l'ONU : un regard africain », in « L'ONU vue d'Afrique », op.cit., p.310. 


\section{CHAPITRE II : LA CONTESTATION DE L'HEGEMONIE DES GRANDES PUISSANCES}

DANS LES ORGANES RESTREINTS

Les petits États contestent de plus en plus l'hégémonie des grandes puissances dans les organes restreints. Cette contestation trouve sa justification dans l'instauration d'un nouvel ordre politique dans les relations internationales. Lordre politique est défini par le Professeur Michel VIRALLY comme "le système de rapports étatiques qui prévaut à une époque donnée et présenté comme comportant des règles de jeu proprement politiques, c'est-à-dire non confirmées ni garanties par des instruments juridiques et n'ayant pas acquis un statut coutumier... » ${ }^{49}$ Dans cette perspective, des reformes structurelles (Section I) et fonctionnelles s'imposent dans les organes restreints (Section II).

449 VIRALLY (Michel), « Panorama du droit international contemporain. Cours général de droit international public», op.cit., p.31 et 32 . 


\section{SECTION I : LA NECESSITE D'UNE REFORME STRUCTURELLE DES ORGANES RESTREINTS}

Il faut entendre par la notion de reformes structurelles, les adaptations de la composition des organes restreints de l'organisation internationale à l'évolution des relations internationales.

Pour le Professeur Riccardo MONACO, «étant donné que le système juridique créé par l'acte constitutif tend à devenir permanent et toujours complexe, il s'ensuit que sa structure même doit s'adapter aux nouvelles exigences de la vie de l'organisation.» ${ }^{450}$

Cette reforme pourrait se matérialiser par lélargissement des organes restreints (Paragraphe I). Toutefois, il convient de souligner que cet élargissement est entravé par de nombreux obstacles (Paragraphe II).

\section{PARAGRAPHE I : L'ELARGISSEMENT DES ORGANES RESTREINTS}

Lélargissement des organes restreints implique l'augmentation du nombre de membres de ceux-ci. A l'analyse, cet élargissement se justifie par la représentativité des petits États dans les organisations internationales (A) et par les mutations politico-économiques survenues dans les relations internationales $(\mathrm{B})$.

\section{A : UN ELARGISSEMENT JUSTIFIE PAR LA REPRESENTATIVITE DES PETITS ÉTATS}

La représentativité des petits États dans les organisations internationales se perçoit à deux niveaux. Il s'agit d'une représentativité démographique, c'est-à-dire qui porte sur le nombre de population qu'ils représentent.

Il est certes vrai que ce sont les représentants des États en général qui siègent dans les organes restreints. ${ }^{451}$ Mais dans la réalité, ceux- ci par le truchement de l'État expriment les aspirations des populations qu'ils gouvernent. De ce fait, la représentation dans les organes restreints devrait être proportionnelle aux nombres d'habitants des différents groupes d'États.

Au Conseil de sécurité des Nations Unies, la représentativité des États au regard de leurs populations est assez significative. En effet, l'ONU a été créée en 1945 par 51 États, dans un monde où le continent européen exerçait un pouvoir colonial sur la majeure partie d'un Sud relativement peuplé. ${ }^{452}$ A cette période les membres permanents comptaient 980 millions d'habitants, soit près

450 MONACO ( Riccardo), «Les principes régissant la structure et le fonctionnement des organisations internationales », op.cit., p.120.

451 En général, les organes interétatiques regroupent les représentants des États. En revanche, les organes intégrés sont composés de personnalités indépendantes.

452 DUMONT (Gérard-François), Editorial paru dans la revue «population et avenir », n66O, novembre 2OO2, www.population-démographie.org., p.3. 
de $40 \%$ de la population mondiale. Mais trois d'entre eux estimaient représenter des populations beaucoup plus importantes. L'URSS se présentait comme le mandant des populations de toutes les Républiques de l’empire soviétique. Quant à la France et le Royaume-Uni, ils se considéraient comme les mandataires des populations de leurs colonies.

Pris dans ce contexte, la représentation privilégiée des cinq membres permanents était admissible. Mais aujourd'hui, plus de 60 ans après la création de l'ONU, cette représentativité démographique des cinq membres permanents est devenue obsolète. Les données démographiques actuelles nous imposent de reconsidérer la composition des organes restreints de l'ONU que sont le Conseil de sécurité et le Conseil économique et social.

La représentativité démographique dont se prévalaient les cinq membres permanents s'est fortement détériorée. Ils comptaient en 2003 environ 1846 millions d'habitants, soit 20\% de la population mondiale. Si désormais on établit une projection moyenne en 2005, la situation laisse entrevoir la poursuite de la diminution de représentativité des cinq membres permanents actuels de 29\%, en 2003, à 26\% en $2025 .{ }^{453}$ Logiquement, les cinq membres permanents ont perdu la représentativité qu'ils avaient à la création de l'ONU.

Le deuxième aspect de la représentativité des petits États est relatif à leur nombre dans les organisations internationales. Avec l'accession à l'indépendance de nombreux États du tiers monde, leur nombre s'est accru dans les organes pléniers. Ils constituent une «majorité numérique» ${ }^{454}$ dont il faut tenir compte dans la composition des organes restreints.

La représentativité démographique combinée à la représentativité numérique des petits États va entraîner des élargissements de certains organes restreints. Le Conseil de sécurité comportait 11 sièges dans sa version de 1945 de la Charte, lorsque les Nations Unies étaient composées de 50 États et depuis un amendement entré en vigueur en 1965, reste formé de 15 membres. ${ }^{455}$

Cet élargissement semble être remis en cause par la majorité des petits États, particulièrement par les États africains. Ceux-ci souhaitent un nouvel élargissement du nombre de membres du Conseil de sécurité. Toutefois, cette reforme souhaitée du Conseil de sécurité suscite de grandes divergences entre les États. Plusieurs formules ont été proposées à ce sujet.

Le projet du G4 (le Brésil, l'Inde, l’Allemagne et le Japon) ${ }^{456}$ qui élargit le Conseil de sécurité à 10 nouveaux membres repartis comme suit : 6 membres permanents (sans droit de véto)

\footnotetext{
453 Ibid, p.3.

454 La majorité numérique s’oppose à la majorité économique. Si la majorité numérique se fonde sur le nombre des États, en revanche la majorité économique tient compte de l'apport matériel des États dans le fonctionnement des organisations internationales.

455 DECAUX (Emmanuel), Droit international public, Dalloz, $7^{\mathrm{e}}$ édition,paris, 2010, p.203.

456 Les États qui soutiennent ce projet sont: Afghanistan, Allemagne, Belgique, Bhoutan, Brésil, Danemark, Fidji, France, Géorgie, Grèce, Haïti, Honduras, Iles Salomon, Inde, Islande, Japon, Kiribati, Lettonie, Maldives, Nauru, Palaos, Paraguay, Pologne, Portugal, République Tchèque, Tuvalu et Ukraine.
} 
et 4 membres non permanents. ${ }^{457}$ La question d'un éventuel droit de véto était réportée pour les 15 prochaines années.

Le projet du groupe « unis pour le consensus » (le Canada, le Pakistan, l'Italie, l'Espagne, la Corée du Sud, l'Argentine, et la Turquie) ${ }^{458}$ beaucoup plus souple qui propose de seulement recommander à l'Assemblée générale d'élire pour une période de 2 ans, 20 membres non permanents, sans aucune augmentation du nombre de membres permanents.

Il y a le projet des États africains. Pour ceux-ci, il existe un trop grand fossé entre le nombre des États africains membres de l'ONU et leur représentation au Conseil de sécurité. Il y a évidemment un écart considérable entre le nombre d'États africains à l'Assemblée générale et leur représentation au Conseil de sécurité. Quatre pays africains sont membres originaires de l'ONU. Il s'agit de l'Ethiopie, de l'Egypte, du Libéria et de l'Union Sud-Africaine. En 1945, lors de la création de «lorganisation mondiale», ${ }^{459}$ l'Afrique avait quatre représentants sur un total de 51 États présents. ${ }^{460}$ A cette époque, la sous représentation de l’Afrique au Conseil de sécurité pouvait se comprendre aisément.

Cependant, les données ont beaucoup évolué. Au début des années 1960 avec l'accélération de la décolonisation, la représentation de l'Afrique à l'ONU s'accentue très rapidement. De 1945 à 1960, la représentation de l'Afrique va passer de 4 à 24 membres pour atteindre 39 en 1971. En 1998, sur les 188 États membres de l'ONU, le groupe des États africains est de 54, le groupe des États d'Asie de 44, d'Europe orientale 26, d'Amérique latine et Caraïbes 34, d'Europe occidentale et autres $26 .{ }^{461}$ Le groupe des États africains compte le plus grand nombre de représentants à l'Assemblée générale de l'ONU. Mais paradoxalement, ce groupe n’est pas suffisamment représenté au Conseil de sécurité.

Pour remédier à cette situation injuste, lors de la Conférence des Chefs d'État et de Gouvernement de l'OUA tenue à HARARE en juin 1997, une déclaration sur la position de l'Afrique par rapport à la réforme du Conseil de Sécurité avait été adoptée. ${ }^{462}$ Ce texte repose sur le constat que l’Afrique est mal représentée au sein du Conseil de sécurité, pour ne pas dire sous représentée. C’est le seul continent qui ne dispose pas de membres permanents au Conseil de sécurité. Ce texte contient donc les propositions des États africains sur lélargissement du Conseil de sécurité

457 Les 6 membres permanents étant composés du G4 et de deux États africains désignés par l'UA. Les 4 membres non permanents seraient à partager entre l'Afrique, l'Asie, l'Europe de l'Ouest et enfin l'Amérique Latine. Les nouveaux membres permanents seraient soumis à une évaluation périodique sur leur capacité à contribuer à la paix, mais pour l'instant les modalités demeurent floues.

458 Les États qui soutiennent ce projet sont: L’Argentine, La Colombie, le Costa Rica, L'Italie, Malte, le Mexique, le Pakistan, la Corée du Sud, San Marin, l' Espagne et la Turquie.

459 Ce terme est le titre de l’ouvrage du Professeur Michel VIRALLY, paru aux Editions Armand Colin à Paris en 1972.

460 DUMONT (Gérard-François), Editorial paru dans la revue «population et avenir », op.cit, p.3.

$461 \quad$ Ibid, p.4.

462 Pour le texte de la déclaration de l'Afrique par rapport à la réforme du Conseil de sécurité, il faut se référer au site internet de l'UA : www.africa-union.org. 
de l'ONU. ${ }^{463}$ Les Chefs d'État et de gouvernement se sont accordés sur le fait que «l'Afrique doit disposer de cinq sièges non permanents dans le Conseil de sécurité élargi». En létat actuel de la composition du Conseil de sécurité, les dix membres non permanents sont répartis de la façon suivante : l'Afrique dispose de trois sièges, l'Asie de deux, l'Amérique latine de deux, l'Europe occidentale et autres de deux sièges et l'Europe orientale d'un siège. L'Afrique disposerait de deux nouveaux sièges non permanents qui ajoutés aux trois autres actuels, feraient un total de cinq membres permanents. ${ }^{464}$

La déclaration de HARARE ne s'arrête pas à la revendication de sièges de membres non permanents. Elle va plus loin dans ses prétentions en réclamant aussi deux sièges de membres permanents. Comme leur nom l'indique, ces deux sièges permanents ne seraient pas soumis au système délection périodique mais seraient occupés de façon permanente par des États africains. L'Afrique rejoindrait au Conseil de sécurité comme nouveaux membres permanents, les anciens membres permanents que sont les États-Unis d’Amérique, la France, le Royaume Uni, la Russie et la République populaire de Chine et les autres nouveaux membres qui seraient élus ou désignés. ${ }^{465}$ Cette revendication des États africains montre que de nouvelles modifications s'imposent dans la configuration du Conseil de sécurité.

La volonté des États africains de reformer la composition du Conseil de sécurité a été réaffirmée dans le cadre de l'UA à travers le « consensus d'Ezulwini » en 2005. L'UA souhaite la même configuration de membres permanents avec deux sièges permanents " avec tous les privilèges et prérogatives de membres permanents y compris le droit de véto ", ainsi que cinq sièges non permanents (Afrique, Asie, Amérique Latine et Europe orientale). ${ }^{466}$ Mais à ce stade de l'analyse, des préoccupations fondamentales méritent dêtre posées : l'Afrique peut-elle assumer les obligations qui découlent du statut de membres permanents du Conseil de sécurité ? En effet, aux termes de l'article 24 de la Charte de l'ONU, «...ses membres confèrent au Conseil de sécurité la

463 Il faut signaler qu’en 1963, le nombre de membres du Conseil de sécurité a été porté de onze à quinze ( article 23 de la Charte) et le nombre des membres du Conseil dont le Vote affirmatif est requis pour toutes les décisions autres que celles de procédure a été porté de sept à neuf y compris les cinq membres permanents (article 27 de la Charte). Ce fut le premier élargissement du Conseil de sécurité de l'ONU. Depuis cette date, la composition du Conseil de sécurité est restée inchangée. Toutefois, par sa résolution 47/62 du 11 décembre 1992 « question de la représentation équitable au Conseil de sécurité et l'augmentation de ses membres ", l'Assemblée générale avait demandé au Secrétaire général des Nations Unies d'inviter les membres à soumettre dans un délai de six mois des observations écrites sur une nouvelle révision du Conseil de sécurité. Egalement en 1993, l'Assemblée générale avait par sa résolution A/ 48/ 26 du 03 décembre, a mis en place un groupe de travail chargé d’examiner tous les aspects de la question. FAYE (André), «Quelle place pour l’Afrique dans un Conseil de sécurité élargi à de nouveaux membres », op. cit., AndreFaye.metawiki.com, p.5.

465 FAYE (André), "Quelle place pour l’Afrique dans un Conseil de sécurité élargi à de nouveaux membres? ", op.cit., p.5.

466 L'Afrique tient fortement à ce que les nouveaux membres permanents aient les mêmes droits que les membres permanents actuels. Ce qui exclut l'approche intermédiaire proposée par certains États. Cette approche créerait une nouvelle catégorie de sièges au Conseil de sécurité avec un mandat qui oscillerait entre trois et quinze ans, selon la proposition, et qui pourrait être sujet à une réélection immédiate. 
responsabilité principale du maintien de la paix et de la sécurité internationales... ${ }^{467}$ Dans les faits, ce sont les membres permanents qui ont en charge cette responsabilité. ${ }^{468}$ Celle-ci entraîne pour eux un certain nombre d'obligations notamment celle de contribuer concrètement à cette mission. ${ }^{469}$

L'ONU est dotée d'un budget qui autorise la perception des recettes et l'ordonnancement des dépenses. Les ressources de l'organisation proviennent d'une part des contributions volontaires qui alimentent des fonds spéciaux créés pour une action spécifique et d’autre part des contributions obligatoires. ${ }^{470}$ De ce fait, tous les États membres de l'organisation sont obligés de verser leur contribution selon un barème spécial. Le critère qui permet de calculer la participation financière de chaque État pour une opération de maintien de la paix est à la fois politique et économique. Laspect politique de ce critère recouvre la responsabilité que les États assument respectivement dans le domaine du maintien de la paix et de la sécurité internationales. Cette responsabilité est plus ou moins grande selon les États membres permanents du Conseil de sécurité et les autres membres de l'organisation. ${ }^{471}$ En effet, l'aspect économique du critère de répartition des contributions financières conduit à accroître les quotes-parts des membres permanents et des pays développés dans le même temps où celles des pays les plus pauvres sont fortement allégées. ${ }^{472}$ Avec la présence de membres permanents africains au Conseil de sécurité, l'Afrique aurait certainement un rôle plus important en matière de maintien de la paix et de la sécurité internationales. Les États africains seraient obligés de participer au financement des opérations de maintien de la paix. Mais, la participation financière que devraient verser les États africains pour le financement des opérations de maintien de la paix pose de véritables problèmes. En vérité, au regard de la situation économique peu reluisante de l'ensemble des États d'Afrique, il est vraiment réaliste de s'interroger si ceux-ci auront les moyens financiers qu'impose le statut de membre permanent du Conseil de sécurité ?

Cette position adoptée par l'Afrique apparaît très intransigeante et teintée de beaucoup d’ambiguités. En effet, l'unité de la position n’est qu’apparente car de nombreux États africains ne sont pas prêts à accepter que certains d'entre eux, deviennent des membres permanents disposant de droit de véto. Il semblerait que cette intransigeance provienne avant tout de la volonté, de ceux qui ne peuvent espérer devenir membres permanents, d’empêcher les États qui seraient susceptibles de le devenir d'acceder à ce statut.

Parallèlement à ces projets de réformes concurrents, Monsieur Kofi ANNAN, alors Secrétaire général de l'ONU, met en branle un processus de réforme en septembre $2003 .{ }^{473} \mathrm{Il}$

\footnotetext{
467 Voir l'article 24 de la Charte de l'ONU.

468 FAYE (André), " Des sièges permanents africains au Conseil de sécurité des Nations unies : pourquoi faire?", AndreFaye.metawiki.com, p.3.

469 Ibid.

470 FAYE (André), « Des sièges permanents africains au Conseil de sécurité des Nations Unies : pourquoi faire?», op.cit., p.10.

471 Ibid.

472 Ibid.

473 MOREAU DEFARGES (Philippe), « La réforme de l'ONU, obsédante et impossible », www.afri-ct.org/ IMG/ pdf/ 67_Moreau_Defarges.pdf, p.1
} 
crée un Groupe de personnalités de haut niveau comprenant 17 membres et présidé par l'ancien premier ministre de la Thailande, Anand PANYARACHUN. Le 2 décembre 2004, le rapport « sur les menaces, les défis et les changements ", " un monde plus sûr : notre affaire à tous ", est remis au Secrétaire général. Le 21 mars 2005, Kofi ANNAN, puisant largement dans le rapport, propose un très ambitieux plan de réforme. ${ }^{474}$ Ce rapport sera débattu en septembre 2005 lors la session extraordinaire de l'Assemblée générale. ${ }^{475}$

Mais quel est le contenu des réformes du Conseil de sécurité proposées par Kofi ANNAN?

On peut remarquer que les propositions du Secrétaire général sont très proches de celles du Groupe de personnalités de haut niveau. ${ }^{476}$ Dans son rapport de mars 2005 intitulé « Dans une liberté plus grande : vers le développement, la sécurité et les droits de l'homme pour tous ", ${ }^{477}$ précisément au point 170, il affirme : «J'engage les États membres à considérer les deux options (formule A et B) proposées dans le présent rapport, ou toute autre proposition viable en termes de nombre et d'équilibre inspirée de l'un ou l'autre modèle. Les États membres devraient convenir de prendre une décision sur une question importante avant le sommet de septembre 2005. Il serait préférable, et de loin, qu'ils prennent cette décision vitale par consensus ; s'ils ne sont pas en mesure de parvenir au consensus, cela ne devra cependant pas servir de prétexte au report de la prise de décision.

Réforme du Conseil de sécurité : formules A et B.

Selon la formule A, il serait créé six nouveaux sièges permanents sans droit de véto, et trois nouveaux sièges non permanents avec mandat de deux ans, répartis entre les principales régions...

La formule B suppose la création de sièges non permanents dont huit avec un mandat renouvelable de 4 ans, et un siège non permanent avec mandat non renouvelable de deux ans. ${ }^{478}$

Ibid. Kofi ANNAN a accueilli avec satisfaction les recommandations du groupe de personnalités et il a approuve l'analyse de l'ensemble du rapport. Il a fait valoir qu'il reprenait à son compte les arguments centraux en faveur du système de sécurité collective plus large et plus global. Dans la note accompagnant ce document, il affirme : « je souscris sans réserve à sa thèse première, à savoir celle d'une sécurité collective à vocation plus étendue et globale qui permette d'appréhender toutes les menaces, anciennes et nouvelles, et les problèmes de sécurité de tous les États -riches et pauvres, faibles et forts-. Selon lui, le rapport, « exhaustif et fouillé » présente « une nouvelle grille d'analyse des liens qui existent entre les différentes menaces et offre l'occasion unique pour remodeler et renover les institutions de l'Organisation des Nations Unies. (Voir TAVERNIER (Paul), « soixante ans après : la réforme du Conseil de sécurité des Nations Unies est-elle possible? », www.ridi.org/adi, août 2005, p.6) Cette session extraordinaire baptisée le « sommet du millénaire » coïncide avec le $60^{\mathrm{e}}$ anniversaire de l’ONU. TAVERNIER (Paul), "Soixante ans après : la réforme du Conseil de sécurité des Nations Unies est-elle possible? ", op.cit.p.7 . Le rapport de Monsieur Kofi ANNAN est disponible sur le site suivant : www.un.org/ french/ largerfreedom. Voir « Dans une liberté plus grande : développement sécurité et respect des droits de l'homme pour tous ", rapport de Monsieur Kofi ANNAN sur la réforme de l'ONU, mars 2005, disponible sur le site suivant : $w w w$. un.org/ french/ largerfreedom. Extraits des pages 49-51. Il est important de souligner que la formule " A " proposée par le Groupe de personnalités de haut niveau semble avoir été conçue pour tenir compte de la demande de l'Afrique de disposer de 2 sièges permanents au sein du Conseil de sécurité. Mais des États africains comme l'Algérie préfèrent plutôt le modèle « $\mathrm{B}$ » le plus démocratique et le plus à même de permettre une rotation équitable. 
Les deux formules $\mathrm{A}$ et $\mathrm{B}$ ont été résumées dans ces deux tableaux suivants :

Model A

\begin{tabular}{lccccc}
\hline Regional area & $\begin{array}{c}\text { Number of } \\
\text { states }\end{array}$ & Permanent seats & $\begin{array}{c}\text { Proposed new } \\
\text { permanent seats }\end{array}$ & $\begin{array}{c}\text { Proposed two-years } \\
\text { seat }\end{array}$ & Total \\
\hline Africa & 53 & (continuing) & & (non renewable) & 6 \\
\hline Asia and pacific & 56 & 1 & 2 & 4 & 6 \\
\hline Europe & 47 & 3 & 1 & 2 & 6 \\
\hline Américas & 35 & 1 & 1 & 4 & 6 \\
\hline Total model A & $\mathbf{1 9 1}$ & $\mathbf{5}$ & $\mathbf{6}$ & $\mathbf{1 3}$ & $\mathbf{2 4}$ \\
\hline
\end{tabular}

model B

\begin{tabular}{lccccc}
\hline Regional area & $\begin{array}{c}\text { Number } \\
\text { of states }\end{array}$ & $\begin{array}{c}\text { Permanent } \\
\text { seats }\end{array}$ & $\begin{array}{c}\text { Proposed new } \\
\text { permanent seats }\end{array}$ & $\begin{array}{c}\text { Proposed two-year seats } \\
\text { (non renewable) }\end{array}$ & Total \\
\hline Africa & & (continuing) & & 4 & 6 \\
\hline Asia and Pacific & 53 & & 2 & 3 & 6 \\
\hline Europe & 47 & 3 & 2 & 1 & 6 \\
\hline Americas & 35 & 1 & 2 & 3 & 6 \\
\hline Totals model B & $\mathbf{1 9 1}$ & $\mathbf{5}$ & $\mathbf{8}$ & $\mathbf{1 1}$ & $\mathbf{2 4}$ \\
\hline
\end{tabular}

Source: United Nation Secretary General report in larger freedom, p.43 (Box 5)

Malgré toutes ces propositions, il faut constater l'échec de la réforme après la réunion de la soixantième session de l’Assemblée générale de l'ONU car aucune décision n’a été prise et aucune solution privilégiée. Cet échec transparaît dans les extraits du document final de cette Assemblée générale du 15 septembre 2005 :

«153. Nous souhaitons - et c'est un élément central de la réforme générale de l'organisation que nous menons - que le Conseil de sécurité soit reformé sans tarder, afin de le rendre plus largement représentatif, plus performant et plus transparent, ce qui accroîtra son efficacité, la légitimité de ses décisions et la qualité de leur mise en œuvre. Nous nous engageons à continuer à nous efforcer d'aboutir à une décision à cette fin, et nous prions l'Assemblée générale d'examiner, d'ici à la fin de 2005, les progrès accomplis sur cette voie.

154. Nous recommandons que le Conseil de sécurité continue à adapter ses méthodes de travail de façon à ce que les États qui n’en sont pas membres participent davantage, le cas échéant, à ses travaux, à ce qu'il réponde mieux de son action devant l'ensemble des États membres et à ce qu'il fonctionnne dans une plus grande transparence. ${ }^{479}$ 
En dehors du Conseil de sécurité, le Conseil économique et social a connu aussi des élargissements. La composition du Conseil économique et social des Nations Unies est passée successivement de 18 à 27 puis 54 membres. Des querelles ont opposé les États du tiers monde et les grandes puissances sur l'universalité et la réforme de cet organe restreint. Pour les petits États, le Conseil économique et social des Nations Unies reste un club où les pays industrialisés continuent à faire sentir leur influence. Il n'est donc pas pleinement représentatif. ${ }^{480}$ En revanche, les membres permanents du Conseil de sécurité craignent que tout élargissement « ouvert » du Conseil économique et social ne soit utilisé comme un précédent qui viserait le Conseil de sécurité luimême et les prérogatives qu'ils ont selon la Charte. ${ }^{481}$ Malgré ces positions contradictoires, la composition du Conseil économique et social des Nations Unies est passée successivement de 18 à 27 puis à 54. Précisons qu’à l’origine, le nombre de membres était de 18 . Il a été élargi à deux reprises. Ce nombre a été porté de 18 à 27 en 1965 puis de 27 à 54 en $1973 .{ }^{482}$

La question de lélargissement du Conseil d’administration de l'OIT a été aussi posée de façon récurrente. Le Professeur Ernest MAHAIM souligne «quà chacune de ses sessions, la Conférence était saisie des réclamations des États extra-européens au sujet de la composition du Conseil.» ${ }^{483}$ A l'origine, le Conseil d'administration de l'OIT se composait 24 membres repartis de la façon suivante : 12 représentent les gouvernements, 6 les patrons et 6 les ouvriers. Le premier élargissement de cet organe restreint eut lieu à la quatrième Conférence en 1922. La composition du Conseil d’administration devrait être modifiée de la façon suivante : il comprenait 32 membres, dont seize représentant les gouvernements, 8 les patrons et 8 les ouvriers. Sa composition a été élargie à 48 membres dont 24 représentent les gouvernements, douze les travailleurs et douze les employeurs. ${ }^{484}$ Mais en 1972, la composition du Conseil d’administration sera modifiée à 56 membres à la suite de l'amendement de 1972.

La représentativité des organes restreints justifie leur élargissement. Mais, les nombreuses mutations politico-économiques survenues dans les relations internationales imposent une recomposition des organes restreints.

480 BAUCHARD (Denis), "Commentaire de l'article 61 de la Charte des Nations Unies ", in Cot (Jean-Pierre), PELLET (Alain), La Charte des Nations Unies, commentaire article par article, $2^{\mathrm{e}}$ édition, p.947.

$481 \quad$ Ibid, p.948.

482 Ibid, p. 943

483 MAHAIM (Ernest), "L’Organisation permanente du travail », RCADI, 1924, III, Tome 4, p.162.

484 MONTCEAU (Marc), L'Organisation internationale du travail, Que sais-je?, nº 836, PUF, 1972, p.28. 


\section{B : UN ELARGISSEMENT IMPOSE PAR LES MUTATIONS POLITICO-ECONOMIQUES DANS LES RELATIONS INTERNATIONALES}

L’environnement politico-économique d'une organisation internationale conditionne largement la configuration de ses organes à un moment donné. Le Professeur René-Jean DUPUY précise que «le dynamisme des organes -d’une organisation internationale- ne peut être isolé de celui des forces politiques qui dominent et orientent l'institution ... etc. ${ }^{485}$

Il s'ensuit que les organes restreints des organisations internationales doivent nécessairement être en adéquation avec les forces politiques qui existent, où qui apparaissent au sein de l'organisation. Lélargissement des organes restreints doit se faire en tenant compte absolument des mutations politiques et économiques opérées dans les relations internationales.

Le cas du Conseil de sécurité de l'ONU constitue un exemple qui pourrait permettre d'expliciter ce point. ${ }^{486} \mathrm{Il}$ est évident que depuis la création de cette organisation internationale, les relations internationales ont connu des bouleversements politiques et économiques majeurs.Ceuxci imposent une recomposition de cet organe restreint. Le Professeur Jean-Pierre QUENEUDEC le souligne en ces termes : «depuis l'entrée en vigueur de la Charte des Nations Unies le 24 octobre 1945, le monde a changé. L’image géopolitique de la planète qui était issue de la seconde guerre mondiale et que l'ONU était vouée à préserver, a été profondément transformée. La fin du monopole nucléaire américain, le démembrement des empires coloniaux britannique et français, la disparition de l'URSS, l'unification allemande, les conquêtes de léconomie japonaise, les prémices de léveil chinois : autant de faits qui ont contribué à modifier léquilibre des puissances au cours du demi-siècle écoulé...» ${ }^{487}$

Manifestement, les réalités politico-économiques de 1945 à nos jours ont connu des mutations substantielles. De nouvelles puissances politiques et économiques ont émergé dans les rapports entre les États. La nouvelle configuration du Conseil de sécurité devrait normalement

485 DUPUY (René-Jean), La communauté internationale entre le mythe et l'histoire, Economica, Paris,1986, p.60.

486 La bibliographie sur cette question est très abondante. Voir notamment : TAVERNIER (Paul), « L'ONU et la réforme du Conseil de sécurité ", in L'ONU, 50 ans après : bilan et perspectives, Colloque organisé par la Faculté de droit de Besançon, 29-30 mars 1995, p.151-174; "La réforme du Conseil de sécurité des Nations Unies. État de la question de la représentation équitable au Conseil de sécurité et de l'augmentation du nombre de ses membres ", Polish Yearbook of international law, 1994, p.41-54; « Réflexion à propos du 50 anniversaire des Nations Unies ", Spécial 50 anniversaire de l'ONU, Centre d'information des Nations Unies et Annuaire français des Nations Unies, Paris, 1995, p.39-43; «La réforme du Conseil de sécurité des Nations Unies : le rôle des grandes puissances ", Revue de recherche juridique, Université Chahid-Behechti (Téhéran-Iran), Faculté de droit, n¹6-17, 1996, p.489-508 (en persan) ; "Faut-il reformer le Conseil de sécurité des Nations Unies? ", p.181-194, in " Un demi-siècle des Nations Unies », Marrakech, Université Cadi Ayyad, série Séminaires et colloques, n7, 1997 ; GUILLOT (Philippe), "La réforme du Conseil de sécurité des Nations Unies ", Arès, Volume XV, ${ }^{\circ} 1$ 1, 1996, p.3-22, FLEURANCE (Olivier), La réforme du Conseil de sécurité. Létat du débat depuis la fin de la guerre froide, Bruylant, Bruxelles, 2000. (Cette bibliographie a été fournie par le Professeur TAVERNIER (Paul), "Soixante ans après la réforme du Conseil de sécurité des Nations Unies est-elle possible? ", Actualité et droit international, www.ridi.org/ adi, Août 2005, p.2) QUENEUDEC (Jean-Pierre), «A propos de la composition du Conseil de sécurité », RGDIP 1995-4, p.955. 
prendre en considération toutes ces évolutions. Il serait logique d'étendre le nombre de membres permanents à de nouveaux États qui constituent aujourd'hui des acteurs importants des relations internationales. Dans les faits, le statut de membres permanents est revendiqué par le Brésil «qui estime qu'un «État du Sud», représentant les pays en voie de développement, doit bénéficier d’un siège de membre permanent au Conseil de sécurité, d’autant que le Brésil est actuellement la onzième puissance économique mondiale.» ${ }^{488}$

A l'automne 2003, dans la foulée de la Conférence de l'Organisation Mondiale du Commerce à Cancun (Mexique), un «triangle» se met en place, comprenant le Brésil, l'Inde et l'Afrique du sud. Ces trois pays demandent officiellement le 25 septembre 2003 une réforme des Nations Unies et des institutions multilatérales, justifiant leurs attitudes par le fait qu'ils sont trois démocraties de trois continents et donc que leurs dirigeants s'expriment au nom des populations qu'ils représentent. ${ }^{49}$

En toute objectivité, un Conseil de sécurité légitime ne devrait pas exclure dans sa nouvelle composition des puissances régionales comme l'Afrique du Sud, le Brésil, l'Inde et le Nigéria. Pour que le Conseil de sécurité puisse pleinement assumer ses fonctions dans une Organisation des Nations Unies rénovée et fonctionnant selon les principes de la Charte, il doit offrir les garanties requises d’objectivité, et c'est de cette exigence là que toute réforme devra tenir compte. ${ }^{490} \mathrm{De}$ ce fait, s'interroge le Professeur DJIENA WEMBOU : «à l'aube du troisième millénaire, le moment n'est-il pas venu de prendre en considération les bouleversements et les changements importants intervenus depuis 1945, sur la scène internationale, dans la composition du Conseil de sécurité ? Peut-on continuer à ignorer les géants du Sud (Brésil, Inde, Nigéria, Afrique du Sud) qui peuvent pourtant jouer un rôle irremplaçable dans la mise en œuvre des dispositions du Chapitre VIII consacrés aux accords et organismes régionaux, et qui font en fait de ces institutions des relais des actions décidées par le Conseil de sécurité ? " ${ }^{491}$

En dehors des nouvelles puissances émergentes, d’autres grandes puissances économiques reconnues dans les relations internationales actuelles méritent de siéger en qualité de membre permanent au Conseil de sécurité. Au nombre de ces États, figurent d’abord l’Allemagne qui est la première puissance économique de l'Europe, et le Japon qui est le deuxième pays contributeur au budget de l'ONU. ${ }^{492}$

Ces deux États qui sont aujourd'hui deux grandes puissances économiques pourraient contribuer davantage aux lourdes charges financières et militaires requises pour les organismes de maintien de la paix de plus en plus coûteuses. A l'heure actuelle, la politique financière des États-

\footnotetext{
488 LE JEUNE (Pierrick), Introduction au droit des relations internationales, LGDJ, 1994, p.96.

489 DUMONT (Gérard-François), Editorial paru dans la revue " population et avenir ", n 660, novembre 2002, www.population-démographie.org., p.3

490 DJIENA WEMBOU (Michel-Cyr), Le droit international dans un monde en mutation, essais écrits au fil des ans,op.cit., p.106.

$491 \quad$ Ibid, p.106.

492 Voir dans les documents annexes.
} 
Unis vis-à-vis de l'ONU est de parvenir dans les années à venir à une réduction importante de la contribution américaine au budget de lorganisation notamment au financement des organismes de maintien de la paix. Les États-Unis affirment que le statut de membre permanent a pour corollaire l'obligation de jouer un rôle actif dans le maintien de la paix internationale. ${ }^{493}$ Pour cette raison, ils soutiennent la candidature de ces deux États au Conseil de sécurité comme membres permanents. Mais, «la réforme du Conseil de sécurité, affirme Madame CHEMILLIER-GENDREAU, ne devrait en aucun cas être seulement un réaménagement de la place des occidentaux (sièges offerts à l'Allemagne et au Japon)». ${ }^{494}$

La composition du Conseil d'administration de l'OIT nécessite une réadaptation dans sa configuration actuelle. La formule malheureuse ${ }^{495}$ des «10 puissances industrielles les plus considérables» présidant la détermination des sièges permanents doit faire lobjet d'un élargissement. ${ }^{496}$ La composition des dix membres permanents ne correspond plus aux nouvelles réalités politico-économiques. ${ }^{497}$ Il est facile de constater que parmi ces dix États ayant un siège de membres permanents, ne figure aucun pays africain. Au moins l'Afrique du Sud qui est la première puissance industrielle du continent pourrait bien disposer d'un siège permanent. Il est absurde que l'Afrique n'ait pas un siège permanent au Conseil d'administration de l'OIT, qui est la tribune par excellence où se prennent les grandes décisions mondiales en matière de législation de travail.

$\mathrm{Au}$ regard de ce qui précède, nous pouvons conclure qu'un élargissement des organes restreints à de nouveaux États s'avère indispensable. Toutefois, cet élargissement souhaité en majorité par les petits États est compromis par de nombreux obstacles.

493 FAYE (André), "Quelle place pour l’Afrique dans un Conseil de sécurité élargi à de nouveaux membres? ", op.cit., p.11.

494 CHEMILLIER-GENDREAU (Monique), "Que vienne le règne de la loi internationale », Le monde diplomatique, janvier 19991, p.19.

495 MAHAIM (Ernest), «Lorganisation permanente du travail», op.cit., p.78.

496 Pour le Professeur Ernest MAHAIM, cette formule a « fatalement pour conséquence de rendre instable la situation des États qui occupent, en importance les dernières places. Il peut arriver que les différences soient très minimes, dans les éléments statistiques correspondant aux critères adoptés, entres les États admis parmi les 10 privilégiés et ceux qui ne sont pas admis. Des modifications très minimes peuvent, d'une élection à l'autre, faire perdre le privilège ou le faire conquérir. » Voir RCADI 1924-III, p.159.

497 Voir l'article 7 de la Constitution de l'OIT. Les membres détenant des sièges non électifs en leur qualité d'États ayant l'importance industrielle la plus considérable à la suite des élections du 6 juin 2008 sont : l'Allemagne, le Brésil, les États- Unis, la Chine, la France, l'Inde, Italie, le Japon, le Royaume-Uni et la Fédération de Russie. 


\section{PARAGRAPHE II : LES OBSTACLES A L'ELARGISSEMENT DES ORGANES RESTREINTS}

Toute réforme, que ce soit dans l'ordre interne ou dans l'ordre international, s'accompagne toujours de heurts. Le concept de réforme signifiant des modifications de méthodes, de pratiques, de procédures, quelquefois de structures, pouvant permettre d'améliorer le fonctionnement d'une organisation ou du système dans son ensemble ne fait pas l'unanimité dans les organisations internationales. ${ }^{498}$ Aussi, lélargissement des organes restreints des organisations internationales est confronté à des difficultés tant techniques (A) que politiques (B).

\section{A : LES OBSTACLES TECHNIQUES}

Deux obstacles techniques entravent l'élargissement des organes restreints. S'il est vrai que l'élargissement remet en cause l'efficacité des organes restreints (1), il faut souligner qu'il est assujetti à une modification des Chartes constitutives des organisations internationales (2).

\section{1) La remise en cause de l'efficacité des organes restreints}

Ainsi que nous l'avons souligné dans nos analyses précédentes, l'une des raisons qui expliquent la création d'un organe restreint est la quête de l'efficacité des organisations internationales.

Cette efficacité des organisations internationales résulte surtout du nombre limité des membres des organes restreints. L'organe restreint, qu'il soit investi d'une fonction exécutive, d'une fonction juridictionnelle ou encore d'une fonction spécialisée, doit être composé d'un nombre limité de membres. On peut donc affirmer que plus est limité le nombre d'États composant l'organe restreint, plus grande en sera l'efficacité. ${ }^{499}$

Lélargissement des organes restreints des organisations internationales pourrait porter atteinte à leur efficacité. En fait, d’une manière générale, le problème de l’efficacité est souvent évoqué pour freiner l'élargissement des organes restreints. ${ }^{500}$ Les États qui s'opposent à l'accroissement du nombre de sièges des organes restreints justifient leur position par la question de l'efficacité. Le Professeur Paul TAVERNIER fait remarquer qu' " une loi de science politique ou de science administrative bien connue veut que si l'on élargit de manière excessive un organe de décision, celui-ci ne peut plus fonctionner efficacement et risque dêtre paralysé ». ${ }^{501}$

498 TAVERNIER (Paul), « Du rapport Bertrand (1985) au rapport du groupe des 18 (1986)», RGDIP, 1998, p.3O9.

499 BOUTROS-GHALI (Boutros), " Le principe dégalité des États et les organisations internationales », op.cit., p.37.

500 BENCHICK (Madjid), « Commentaire de l’article 33 de la Charte de l'ONU », op.cit., p.445.

501 TAVERNIER (Paul), «Soixante ans après: la réforme du Conseil de sécurité des Nations Unies est -elle possible? » Actualité et droit international, www.ridi.org /adi, aoûte 2005, p.8. 
En pratique, ce point de vue est confirmé par les problèmes engendrés par l'élargissement successif du Conseil économique et social de l'ONU. Selon le Professeur Emmanuel DECAUX, « par contraste, le Conseil économique et social peut sembler un organe "semi-restreint " avec ses 54 membres, élus pour des mandats de trois ans par l’Assemblée générale, surtout si l'on se rappelle qu'avant cet élargissement entré en vigueur en 1973, ce nombre était de 18 dans le texte initial de la Charte, puis de 27 lors d'une première reforme en 1965. Le Conseil économique et social, organe principal placé sous l'autorité de l'Assemblée générale se trouve dès lors dans une situation bâtarde, ne pouvant disposer ni de la représentativité de l'Assemblée générale, ni de l'efficacité du Conseil de sécurité. $»^{502}$ La taille du Conseil économique et social nuit à son efficacité. Une réduction de sa composition s'impose avec acuité..$^{503}$

Le précédent créé par lélargissement du Conseil économique et social a fortement influencé celui du Conseil de sécurité. Les principales objections à l'idée d’un Conseil de sécurité élargi ont porté sur la perte de son efficacité. Pour les membres permanents actuels, « il n’est pas opportun d'accroître le Conseil dont la composition actuelle constitue [...] le maximum compatible avec le bon accomplissement de ses fonctions.» ${ }^{504}$ L'organisation du travail est plus difficile dans un organe composé d'un nombre important de membres. Les débats sur la question à lordre du jour et les prises de décision se prolongent nécessairement lorsque lorgane restreint est élargi de façon exponentielle.

Si les États-Unis d'Amérique ont accepté le principe d'un élargissement, il faut noter que leur acceptation est assujettie à de nombreuses conditions. Ils proposent un Conseil de sécurité de 20 membres ou de 21 membres au plus. Lélargissement du Conseil doit être limité afin de préserver l'efficacité dudit conseil. ${ }^{505}$ La crainte que nourrissent les États, particulièrement les membres permanents, est de voir une augmentation du nombre des membres se faire au détriment de l'efficacité du Conseil. Le Conseil de sécurité pourrait-il prendre efficacement des décisions? Sa capacité à prendre des décisions avec promptitude pour répondre à un besoin urgent ne risque-telle pas d'être grandement affaiblie?

Lélargissement des organes restreints pose un véritable dilemme dans le fonctionnement des organisations internationales. Il obéit à une volonté de démocratisation des organes restreints. Pour ceux-ci, l’enjeu de cette question réside surtout dans la représentativité de cet organe. En revanche, s'interroge le Professeur DEGNI-SEGUI : « ce que l’on gagnerait en idéal démocratique, ne la perdrait-on pas en efficacité ? » ${ }^{506}$ Entre ces deux extrêmes, il faut sorienter vers une solution

\footnotetext{
502 DECAUX (Emmanuel), Droit international public, op. cit., p.202.

503 NOVOSSELOFF (Alexandra), «L'ONU ou la réforme perpétuelle », AFDI, 2004, p.542.

504 MANKIEWICZ ( R.H), « Augmentation des membres de l'OACI », op .cit., p.447.

505 FAYE (André), «Quelle place pour l'Afrique dans un Conseil de sécurité élagi à de nouveaux membres », op.cit., p.11.

506 DEGNI-SEGUI (René), "Commentaire de l'article 24 paragraphe 1 et 2 de la Charte des Nations Unies », in COT (Jean-Pierre), PELLET (Alain), La Charte des Nations Unies, commentaire article par article ", $2^{\mathrm{e}}$ édition, op.cit., p.465.
} 
de compromis qui consisterait à augmenter le nombre de membres des organes restreints dans une proportion raisonnable.

Mais dans tous les cas, cette augmentation du nombre de membres passe nécessairement par la modification des chartes constitutives des organisations internationales.

\section{2) La modification de la Charte constitutive des organisations internationales}

La charte constitutive d'une organisation internationale revêt une double nature juridique. Elle est un traité international, fondé, en tant que tel, sur la volonté des contractants. ${ }^{507}$ En outre, elle a la valeur d'une Constitution en tant quacte fondateur de l'institution internationale. Pris dans sa seconde acception, c'est-à-dire comme loi fondamentale, la charte constitutive définit les pouvoirs et la composition de l'organisation internationale. Toute modification de la structure initiale d'un des organes de l'organisation entraine sa modification.

Tout élargissement d'un organe restreint est soumis à une modification ou un amendement préalable de la Charte constitutive des organisations internationales. ${ }^{508}$

507 MONACO (Riccardo), « Les principes régissant la structure et le fonctionnement des organisations internationales », op.cit., p.101.

508 Réviser la Charte des Nations Unies signifie que des transformations devront être apportées à l'Acte constitutif. Plusieurs termes peuvent être employés pour désigner les transformations qu’un traité peut subir. On parle alors d'amendement, de révision, de modification. La Commission de droit international dans lélaboration de ses projets d'articles avait retenu le terme " amendement " pour les modifications mineures et celui de « révision » pour les transformations fondamentales. Elle a finalement écarté le terme " révision » et retenu celui d'amendement pour désigner les modifications concernant toutes les parties et celui de "modifications " lorsque seules certaines parties se sont entendues pour conclure un accord modifiant le traité ou lorsque la pratique des États a transformé le régime du traité.

La Convention de Vienne sur le droit des traités de 1969 a consacré cette conception dans sa quatrième partie réservée aux amendements et modifications des traités. Toutefois le principe dominant en matière de révision des traités est celui de l'accord entre les parties, que la révision soit envisagée en l'absence de toute clause conventionnelle la prévoyant, ou lorsqu'une telle clause existe. Ce principe est expressément prévu à l’article 39 de la Convention de Vienne en ces termes: «Un traité peut être amendé par accord entre les parties. Sauf dans la mesure où le traité en dispose autrement, les règles énoncées dans la partie II s’appliquent à un tel accord. » Par l'invocation des dispositions contenues dans la deuxième partie de la Convention de Vienne concernant les règles générales de la conclusion des traités, la Convention pose implicitement le principe de l’absence d’obligations générales pour toutes les parties au traité de participer à l'accord d’amendement. On admet également que l'accord d'amendement peut, lorsque le traité ne se prononce pas, prendre n'importe quelle forme, orale ou tacite. Dans la sentence arbitrale rendue le 22 décembre 1963 entre la France et les États -Unies d'Amérique à propos de l'interprétation de leur accord bilatéral de transport aérien, il est tenu compte de la pratique ultérieure des parties considérée non seulement comme un moyen utile d'interprétation de l'accord mais aussi comme source d'une modification postérieure de celui-ci.

Linsertion de clauses de révision dans les traités multilatéraux revêt une importance particulière dans les relations conventionnelles. Elle a pour effet de faciliter les nécessaires adaptations des traités internationaux et permet une diversification des relations contractuelles. Dans les traités bilatéraux, toute initiative de révision est perçue comme une offre de renégociation du traité. L’utilisation fréquente des clauses de révision a progressivement permis dériger en règles certaines pratiques répandues en matière de révision. Il en ainsi pour les règles relatives aux Conventions qui ne peuvent être révisées qu’après l’écoulement d’un certain délai. 
Les articles 108 et 109 de la Charte des Nations Unies posent les conditions de toute modification. ${ }^{509}$ Larticle 108 de la Charte dispose que "les amendements à la présente Charte entreront en vigueur pour tous les membres des Nations Unies quand ils auront été adoptés à la majorité des deux tiers des membres de l’Assemblée générale et ratifiés, conformément à leurs règles constitutionnelles respectives, par les deux tiers des membres de l'organisation, y compris tous les membres permanents du Conseil de sécurité.» Quant à l'article 109 il prévoit que «... toute modification à la présente Charte recommandée par la Conférence à la majorité des deux tiers prendra effet lorsqu'elle aura été ratifiée, conformément à leurs règles constitutionnelles respectives, par les deux tiers des membres des Nations Unies, y compris tous les membres permanents du Conseil de sécurité...» Cette idée est reprise par Nicoletta PIROZI et Natalino RONZITTI en ces termes : "Any modification of the United Nations Security council involves an amendement of the United Nation Charter [....] The Charter sets out two mechanisms : an amendement procedure (article 108) and a review procedure (article 109). From a formal standpoint there is no difference between the two procedures as far as amending the Charter is concerned. $»^{510}$

Il découle de ces deux dispositions que toute modification de la Charte des Nations Unies exige deux conditions : «Any modification must obtain two-thirds of the votes of General Assembly or of the rewiew Conference and must be ratified by two-thirds of the United Nations members, includind the permanent members of the Security council $»{ }^{511}$ Laccord de tous les membres permanents du Conseil de sécurité est donc nécessaire pour toute reforme du Conseil de sécurité. ${ }^{12}$ Or dans le processus décisionnel au sein de cet organe, les membres permanents disposent d'un privilège aristocratique qu'est le droit de véto. Chacun des cinq membres permanents peut sopposer par son droit de véto à tout projet délargissement du Conseil de sécurité.

Ces deux dispositions constituent un autre grand obstacle au changement de la taille et de la composition du Conseil de sécurité. ${ }^{513}$ Tout changement dans la composition du Conseil de sécurité doit se faire par un amendement de la Charte. Or selon l'article 108, pour qu'un amendement puisse entrer en vigueur, il doit avoir été adopté à la majorité des deux tiers des membres de l’Assemblée générale et ratifiée par les deux tiers des membres de l'organisation, y compris tous les membres du Conseil de sécurité. ${ }^{514}$

Pour Monsieur HAZBI, «en ces articles se cristallisent, plus sans doute qu'en aucun autre article de la Charte, les objectifs contradictoires recherchés par les grandes puissances.

\footnotetext{
509 Voir les articles 108 et 109 de la Charte des Nations Unies.

510 PIROZZI (Nicoletta), RONZITTI (Natalino), " The European Union and the reform of the United Nations Security council : Toward a new regionalism?, Istituto affari internazionali, working paper 11, may, 2011, p.4. 511 Ibid.

512 FAYE (André), "Quelle place pour l’Afrique dans un Conseil de sécurité élargi à de nouveaux membres? ", op.cit., p.17.

513 HATTO (Ronaldo), ONU et maintien de la paix : propositions de réforme. De l'Agenda pour la paix au Rapport BRAHIMI, L'Harmattan, Paris, 2006, p.55. Ibid, p.296.
} 
Traité constitutif d'une organisation internationale devant servir de fondement juridique à la société internationale toute entière, la préserver des dangers de la guerre moderne et encadrer ses inéluctables mutations, la Charte devait pouvoir être modifiée [...] mais ces modifications ne devaient porter atteinte à léquilibre fragile de forces et de responsabilités... ${ }^{515}$ Ces deux dispositions introduisent une inégalité fondamentale au profit des cinq membres permanents. Chacun d'eux possède un pouvoir discrétionnaire de blocage, nonobstant le principe de l'égalité souveraine hautement réaffirmé par l'article 2, paragraphe 1 , de la Charte. Les cinq membres permanents restent maîtres de la procédure d’amendement et leur assentiment est la condition sine qua non de toute modification de la Charte. ${ }^{516}$

Le pouvoir discrétionnaire dont dispose les cinq membres permanents du Conseil de sécurité suscite des interrogations. En fait, est-ce que ceux-ci sont réellement disposés à accepter un rééquilibrage des rapports de force au sein du Conseil de sécurité, ou sont-ils disposés à partager avec d'autres membres les privilèges issus de la seconde guerre mondiale ?517 Les enjeux véritables de toute ratification de la Charte des Nations Unies visant l'élargissement du Conseil de Sécurité se situent à ce niveau.

Le privilège dont bénéficient les grandes puissances dans le processus d’amendement de la Charte constitutive des organisations internationales a été prévu par la Constitution de l'OIT. Aux termes de l'article 36, il est formellement prescrit que «les amendements à la présente Constitution adoptés par la Conférence à la majorité des deux tiers des suffrages émis par les délégués présents entreront en vigueur lorsqu'ils auront été ratifiés ou acceptés par les deux tiers des membres de lorganisation comprenant cinq des dix membres représentés au Conseil d'administration en qualité de membres ayant l'importance industrielle la plus considérable, conformément aux dispositions du paragraphe 3 de l'article 7 de la présente Constitution.» ${ }^{518}$ Cette disposition implique l'accord nécessaire de cinq des dix puissances industrielles représentées au Conseil d’administration avant tout amendement visant l'augmentation de ses membres. Elle traduit la volonté des rédacteurs de la Constitution de cette organisation à subordonner toute modification de l'Acte constitutif à l'unanimité d’au moins cinq puissances industrielles siégeant au Conseil d’administration.

La révision de la Charte constitutive des organisations internationales constitue une question délicate, à la limite une question tabou. ${ }^{519}$ Les obstacles techniques à franchir pour lélargissement des organes restreints des organisations internationales sont réels. Mais, ils ne doivent pas occulter les contraintes politiques.

\footnotetext{
515 HAZBI (Aziz), ONU et ordre mondial: réformer pour ne rien changer, op.cit., p.76.

516 FLEURENCE (Olivier), La réforme du Conseil de sécurité. Létat du débat depuis la fin de la guerre froide, Bruylant, Bruxelles, 2000, p.16.

517 FAYE (André), "Quelle place pour l'Afrique dans un Conseil de sécurité élargi à de nouveaux membres ? ", op.cit., p17.

518 Voir l'article 36 de la Constitution de l'OIT.

519 TAVERNIER ( Paul), « Le processus de réforme des Nations Unies. Du rapport Bertrand (1985) au rapport du groupe des 18 (1986)», RGDIP 1988, p.324.
} 


\section{B : LES OBSTACLES POLITIQUES}

En règle générale, toutes les réformes peuvent s'enliser ou dépérir avant même d'avoir pu sépanouir en raison de facteurs politiques. ${ }^{520}$ Aussi, deux considérations politiques freinent-ils lélargissement des organes restreints. D’abord, il est important de souligner le bouleversement des équilibres politiques au sein de ces organes (1), ensuite l'incompatibilité du modèle démocratique étatique dans les organisations internationales (2).

1) Le bouleversement des équilibres politiques au sein des organes restreints.

Comme l'a souligné le Professeur Charles CHAUMONT «les organes d'une organisation répondent à certaines exigences et à certaines transactions qui se concrétisent au bout d'une longue et difficile négociation». ${ }^{51}$ La configuration des rapports de force interne dans un organe restreint obéit à des considérations politiques recherchées par les membres originaires de l'organisation.

Lélargissement des organes restreints pourrait dans une certaine mesure remettre en cause ces rapports de force. C'est ainsi que la question de lélargissement du Conseil de sécurité donne un reflet particulier aux critères de composition de cet organe. Il apparaît aux grandes puissances, que «l'institutionnalisation» de la notion de démocratisation des relations internationales porte en germe une évolution de l'équilibre des forces que les membres permanents risquent de ne pas pouvoir contrôler. ${ }^{522}$

Inéluctablement, un élargissement du Conseil de sécurité entrainera un bouleversement considérable des forces politiques au sein de cet organe. D’où le consensus négatif des cinq membres permanents contre tout élargissement du Conseil, bien que le nombre des États membres de l'ONU ait été trois fois plus élevé qu'en $1945 .^{523}$

Les candidatures des États déclarées pour un siège de membre permanent suscitent bien souvent des oppositions fondées en général sur des raisons politiques. Ainsi, la ratification par la Chine d’une réforme du Conseil de sécurité qui accueillerait le Japon comme membre permanent avec droit de veto est impensable pour le moment. ${ }^{524}$ De fait, la cohabitation de ces deux voisins a été toujours marquée par des rivalités politico-militaires très anciennes. La logique veut que la Chine ne donne pas à un tel voisin plus de puissances qu'il n'en détient. ${ }^{525}$

520 TAVERNIER (Paul), « Le processus de réforme des Nations Unies. Du rapport Bertrand (1985) au rapport du groupe des 18 (1986), op.cit., p .330.

521 CHAUMONT (Charles), "Léquilibre des organes politiques des Nations Unies et la crise de l'organisation, op.cit., p.429.

522 BENCHICK (Madjid), « Commentaire de l'article de l'article 33 de la Charte des Nations Unies », op.cit.p.439.

523 TAVERNIER (Paul), " soixante ans après : la réforme du Conseil de sécurité des Nations Unies est -elle possible ?», Actualité et droit international, www.ridi.org/adi, août 2005, p.4.

524 FAYE (André), Quelle place pour l’Afrique dans un Conseil de sécurité élargi à de nouveaux membres? », op. cit.p.10.

Ibid. 
La candidature de l'Allemagne, si elle était acceptée, soulèverait incontestablement un problème de leadership politique entre ce pays et la France en Europe. Ladmission de l'Allemagne au Conseil de sécurité comme membre permanent permettrait à ce pays d’acquérir une très grande puissance sur la scène internationale et en Europe, en particulier. Ce statut pourrait permettre à l'Allemagne d'occuper une place centrale au sein de l'Union européenne et y jouer les premiers rôles. Mais, ceci n’est pas sans inquiéter certains États Européens, notamment la France car la puissance de l'Allemagne éveille des craintes. ${ }^{526}$ Dans ces conditions, est-que la candidature de l'Allemagne au Conseil de sécurité peut-elle être soutenue par l’ensemble des États européens ?

La position de la Russie est intimement liée à ses difficultés politiques et économiques actuelles. De ce fait, la Russie est-elle prête à partager avec d'autres États le seul symbole de la puissance qui lui reste aujourd'hui ? $^{527}$ En effet, sur la scène internationale, un nouvel ordre politique international unipolaire s'est mis en place. Il résulte de l'autodestruction du système socialiste qui a succombé, victime de ses propres contradictions ${ }^{528}$. Lanalyse des données politiques internationales donne de constater que la Russie ne représente plus la grande puissance rivale des États-Unis d’Amérique. Depuis 1990, la Russie connaît une série d'échecs sur le plan politique. C’est d’abord l'échec d'un système de fédération qui se voulait intégratif ; l'échec d'une fédération qui se voulait centralisatrice. Cette situation a conduit de nombreux États de la Fédération à prendre de plus en plus de distance vis-à-vis de la Fédération de la Russie. Cette situation qui est le premier élément de la délégitimation de la Fédération constitue un facteur essentiel de contestation du système mis en place. C’est pourquoi depuis un certain nombre d'années, l’on assiste progressivement à la désagrégation de la Fédération de la Russie.

La Russie ne compte encore dans les relations internationales que grâce à son statut de membre permanent du Conseil de Sécurité, même si l'on n’ignore pas sa force nucléaire. ${ }^{529}$ Elle tient aujourd'hui une place privilégiée au plan international en raison de son statut de membre permanent du Conseil de sécurité de l'ONU. L’arrivée de nouvelles puissances économiques (Japon, Allemagne...) au Conseil de sécurité reléguerait la Russie à une échelle inférieure. Logiquement, dans sa proposition de réforme du Conseil de sécurité, la Russie ne semble pas disposée à l'accroissement des membres permanents. ${ }^{530}$

Ces divergences et ces difficultés montrent qu'au-delà des déclarations favorables à la reforme de l'ONU, la mise en œuvre de celle-ci demeure très problématique. ${ }^{531}$ Le syndrome de

526 Ibid.

527 FAYE (André), "Quelle place pour l'Afrique dans un Conseil de sécurité élargit à de nouveaux membres ", op.cit., p.10.

528 LAGMANI (Slim), « Le nouvel ordre politique international et son impact sur le droit international », in Affaires et documents de droit international, Centre de Publication Universitaire, Tunis, 2005, p.10.

529 FAYE (André), "Quelle place pour l'Afrique dans un Conseil de sécurité élargi à de nouveaux membres ", op.cit., p.10.

530 FAYE (André), « Quelle place pour l’Afrique dans un Conseil de sécurité élargi à de nouveaux membres ? »,op. cit.,p.10.

531 HAZBI (Aziz), ONU et ordre mondial: réformer pour ne rien changer, op.cit., p.129. 
l'État voisin, frère ennemi, se dévoile et porte l'irrationalité jusqu’à saboter toute réforme pour ne pas laisser accéder le rival à une position qui en soi traduit la revendication de démocratisation des relations internationales tant attendues. ${ }^{532}$

Mais, il est important de souligner qu'avec l’adoption par l'Assemblée générale de la résolution 48/26 du 3 décembre 1993, en vertu de laquelle fut créé le groupe de travail sur l'élargissement du Conseil de sécurité et la réforme de ses méthodes de travail, il y a eu une évolution qui montre une certaine ouverture au sein de l'ONU où on accepte désormais de débattre de l'élargissement et de l'efficacité du Conseil de sécurité. ${ }^{533}$ Mais, cette semi-ouverture n’a pas encore abouti à des décisions concrètes. Toutefois, il semble désormais acquis que la composition du Conseil de sécurité devrait refléter certains changements intervenus dans le monde. ${ }^{534}$ Ce point a été suffisamment appuyé lors du sommet du millénaire. La déclaration du millénaire du 13 septembre 2000 issue de ce sommet consacre une partie de ses développements à cette question..$^{535}$

Plusieurs puissances se sont, depuis, déclarées favorables à la réforme de l'ONU. Dans un communiqué commun, le Sommet franco-britannique du 18 novembre 2004 a exprimé le soutien de la France et du Royaume-Uni à « une réforme du Conseil de sécurité qui adaptera sa composition aux réalités d’aujourd'hui. ${ }^{536}$ La France s'est donc déclarée favorable à un élargissement du Conseil de sécurité, aussi bien en ce qui concerne le nombre des membres permanents que celui des membres non permanents. ${ }^{537}$ La France soutient l'accession à un siège de membre permanent de l'Allemagne, du Brésil, de l'Inde et du Japon. Elle appuie aussi une présence plus importante des pays africains au Conseil de sécurité, notamment parmi les membres permanents. ${ }^{538}$ Les États-Unis semblent se rallier à la réforme du Conseil de sécurité, ${ }^{539}$ à leurs conditions, et sans que ce soit une

$532 \quad$ Ibid, p.130

533 Ibid, p.91.

534 Ibid.

535147 Chefs d'État et de gouvernement, sur les 189 États membres, représentant les pays riches et les pays du sud qui regroupent près de la moitié des 6 milliards de la planète, vivant avec moins de deux dollards par jour, ont participé du 6 au 8 septembre 2000, à New York, au Sommet du Millénaire. La réunion s’est terminée par la « déclaration du millénaire » qui a fait l’objet de la résolution 55/2 de l’Assemblée générale de l’ONU, en date du 8 septembre 2000 .

536 Voir le site internet suivant : www.un.int/France.

537 Ibid.

538 A ce sujet, l’ex- Président de la République française Nicolas SARKOZY a déclaré le 30 janvier 2011 au Sommet de l'Union africaine à Addis-Abeba (Ethiopie) : «Je suis convaincu depuis bien longtemps que l'Afrique n'a pas la place qui lui revient dans la gouvernance internationale. Alors, puisque le Président de l’Assemblée générale des Nations Unies est ici et que le Secrétaire général est ici, je leur dis : faites la réforme du Conseil de sécurité dès cette année. N'attendez pas. La France vous soutiendra. Ne faites pas un discours, prenez des décisions. Elargissez le nombre de membres du Conseil de sécurité, reconnaissez au milliard d'africains la place à laquelle ils ont droit et la France vous soutiendra...» (Voir La France à l'ONU, www.un.int/France)

539 Déclaration de la déléguée des États-Unis, communiqué de presse AG/ 10338 relatif aux $87^{\mathrm{e}}$ et $88^{\mathrm{e}}$ séances plénières de l’Assemblée générale, du 7 avril 2005. 
priorité pour eux. Ils insistent plutôt sur la rationalisation de la gestion de l'organisation. ${ }^{540}$ Quant à la Chine, elle se contente de se prononcer en faveur d'un large consensus sur la réforme de l'ONU. ${ }^{541}$

Comme on peut le constater, la position des grandes puissances sur la réforme du Conseil de sécurité a évolué favorablement.

La démocratisation du Conseil d'administration de l'OIT est aussi confrontée à des problèmes politiques résultant de la remise en cause des rapports de force entre les États. Sa réforme s'est posée en des termes différents que celle du Conseil de sécurité de l'ONU. Ainsi, invoquant la démocratisation du Conseil d’administration, les pays en voie de développement demandent la suppression des dix sièges permanents, attribués depuis l'origine aux dix puissances industrielles. ${ }^{542}$ Vraisemblablement, cette requête des pays en développement pose le problème de l'équilibre des forces politiques au sein du Conseil d'administration de l'OIT. Le concept de puissances industrielles utilisé afin de légitimer les dix sièges permanents octroyés aux États industrialisés traduit une certaine réalité politique internationale. Les États industrialisés n’ont pas les mêmes niveaux de développement économique, social et technologique que les États en développement. Or, la préoccupation principale des fondateurs de l'OIT est aisée à comprendre, et elle est justifiée. Il faut que la direction de lorganisation reste dominée par les États dont les plus grands intérêts sont engagés, les États les plus importants au point de vue industrielle. ${ }^{543}$ Il en résulte que l'orientation politique de l'OIT en général et, du Conseil d’administration en particulier relève globalement des initiatives de ces grandes puissances industrielles. La revendication des pays en développement tendant à supprimer ces dix sièges permanents ne sera pas sans conséquences sur la configuration des forces politiques au sein de cet organe restreint.

Leur suppression conduirait à traiter sur un pied de stricte égalité les pays industrialisés et les pays en développement au Conseil d'administration. Cette remise en cause des rapports de force entre les grandes puissances et les petits États rend presqu'impossible la démocratisation voulue par les petits États. Objectivement, pour le Professeur Paul TAVERNIER, « aucune réforme n’a de chance de réussir si elle trop radicale et fondamentale. Seule une réforme progressive, qui ne bouleverse pas l'ensemble de l'édifice est acceptable». ${ }^{544}$

Le bouleversement des équilibres politiques existant entre les États membres constitue l'un des obstacles à la démocratisation des organes restreints des organisations internationales. Cependant au-delà de ces écueils, un point essentiel mérite d’être souligné. Les Etats parlent de plus en plus de démocratisation des organes restreints. Mais de quelle démocratie s’agit-il ? Le modèle

\footnotetext{
540 HAZBI (Aziz), ONU et ordre mondial, op.cit., p.94.

$541 \quad$ Ibid.

542 DUPUY (Béatrice), Réforme de structure et démocratisation de l'OIT, travaux de recherche de l'institut du droit de la paix et du développement, Nice, 1979, p.175.

543 MAHAIM (Ernest), « Lorganisation permanente du travail », op.cit., p.159.

544 TAVERNIER (Paul), « Le processus de réforme des Nations Unies. Du rapport Bertrand (1985) au rapport du groupe des 18 », RGDIP, 1988, p.326.
} 
de démocratie valable dans l'ordre interne des Etats est-il incompatible avec celui des organisations internationales?

2) L'incompatibilité du modèle démocratique étatique au sein des organisations internationales

La démocratie dans l'État doit-elle avoir le même sens et contenu que dans les organisations internationales? Autrement dit, les États peuvent-ils transposer le modèle de démocratie qui prévaut dans leurs ordres internes dans les organisations internationales?

La réponse à cette question peut véritablement nous situer sur les enjeux et le réalisme de la démocratisation des organes restreints des organisations internationales. Il est vrai que l'une des exigences du nouvel ordre politique revendiqué par les petits États est la démocratisation des relations internationales. Mais, il faut reconnaître la différence qui existe entre la démocratie dans lordre interne des États et celle qui est revendiquée dans les organisations internationales.

La démocratie est conformément à son étymologie, le pouvoir du peuple, par le peuple et pour le peuple. Cette définition de la démocratie constamment rappelée dans la plupart des Constitutions des États modernes en général et dans les États africains en particulier, traduit à elle seule le fait que le pouvoir au sein de l'État appartient au peuple. Ce sont les individus qui sont les titulaires du pouvoir et qu'ils confient à des autorités qu'ils élisent.

Cette conception de la démocratie n'est pas transposable dans l'ordre international, et particulièrement dans les organisations internationales.

Dans l’ordre international il est facile de remarquer la grande disparité qui prévaut au plan matériel entre les États. La classification entre les États oppose les riches aux États pauvres. ${ }^{545}$ Les États dans l'ordre international n’ont pas tous le même niveau de développement économique, politique et social. Le principe d'égalité entre les États n’est que formel, car dans les faits les États membres de la communauté internationale ne sont pas matériellement égaux.

La logique juridique qui sous-tend la vie politique internationale est très différente de celle de l'ordre interne. Alors que la vie politique au sein de l'État est régie par un droit de subordination qui conditionne des sujets susceptibles dêtre contraints au besoin par la force, grâce à un appareil institutionnel adéquat, à l'observance des lois; la vie politique internationale est encadrée par un droit de coordination qui se borne à favoriser la coopération entre les États. ${ }^{546}$ Le pouvoir politique dans l'ordre interne est règlementé par des autorités politiques qui sont tenus de faire respecter la légalité. A l'opposé, souligne le Professeur DUPUY «dans l'ordre international, les États nétant couverts par aucune autorité de superposition, ne se relient les uns aux autres que sur la base volontaire et demeurent chacun souverain dans lestimation de leur droit. C'est dire que

545 DUPUY (René-Jean), La communauté internationale entre le myIthe et l'histoire, Paris, Economica, 1986, p.11O.

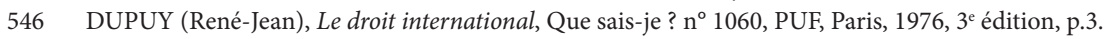


la règle de droit n'est pas toujours comprise de la même façon par tous et les États ont tendance à hypostasier en valeurs sacrées leurs intérêts les plus importants.» ${ }^{547}$

Au regard de ce qui précède, il est évident que la démocratie telle qu’elle fonctionne au sein de l'État ne peut être reproduite dans les organes restreints des organisations internationales. La logique qui sous-tend la mise en place d'un organe restreint dans une organisation internationale fonde la nécessité d’une configuration des forces politiques assez particulière. Pour le Professeur Charles CHAUMONT «les organes restreints des organisations internationales, compétents pour des cas concrets, il faut la concentration de la puissance (avec les membres permanents) dans une suffisante représentation des continents (avec les membres non permanents).» ${ }^{58}$ Dans cette perspective, l'objectif poursuivi à travers l'institution des organes restreints est la recherche d'un organe «exécutif fort» dans les organisations internationales. Le nombre restreint de ses membres, la puissance qu'ils représentent et la place prépondérante des intérêts des grandes puissances orientent ces organes vers l'action. ${ }^{549}$

Dans ces conditions, la démocratie au sein des organes restreints doit prendre en considération la puissance politique des grands États et la majorité numérique des petits États. Lélargissement des organes restreints doit assurer une représentation considérable des plus importants contributeurs de l'organisation et permettre une représentation équitable des petits États en raison de leur nombre.

C'est donc à juste titre que le Professeur Jean-Pierre QUENEDEUC note que «concevoir le problème de la représentativité comme un problème quasi mathématique, avec la part inévitable d’alchimie que recèlent de délicats dosages de représentation, c'est sans doute s'engager dans une impasse». ${ }^{550} \mathrm{Si}$ des reformes structurelles s'avèrent indispensables pour la démocratisation des organes restreints, il faut aussi mentionner l'apport inestimable des reformes fonctionnelles en vue de corriger les inégalités entre les États membres dans les organisations internationales.

\section{SECTION II : L'EXIGENCE DE REFORMES FONCTIONNELLES DES ORGANES RESTREINTS.}

Contrairement aux reformes structurelles qui concernent la taille et la composition des organes restreints, les reformes fonctionnelles sont relatives aux aspects fondamentaux du fonctionnement des organisations internationales. Dans cette perspective, la revendication par les petits États de la suppression du droit de véto et du statut de membres permanents des grandes

$547 \quad$ Ibid.,p.3 et 4.

548 CHAUMONT (Charles), "Léquilibre des organes politiques des Nations Unies et la crise de l'organisation", AFDI 1965, p.433.

549 FLEURENCE (Olivier), La réforme du Conseil de sécurité, létat du débat depuis la fin de la guerre froide, Bruylant, Bruxelles, 2000, p.222.

550 QUENEDEUC (Jean-Pierre), «A propos de la composition du Conseil de sécurité », op.cit, p.958. 
puissances mérite une analyse approfondie (Paragraphe I). Mais, sur cette question la position originale de l'UA à travers le Conseil de paix et de sécurité doit être mise en relief dans le cadre de cette étude (Paragraphe II).

\section{PARAGRAPHE I : LA REVENDICATION DE LA SUPPRESSION DU DROIT DE VETO ET DU STATUT DE MEMBRES PERMANENTS PAR LES PETITS ÉTATS}

Dans les organisations internationales, les petits États remettent en cause les privilèges fonctionnels reconnus aux grandes puissances. Parmi ceux-ci, figurent le droit de véto et le statut de membres permanents. Si cette revendication semble être justifiée du point de vue du droit (A), dans les faits elle paraît difficilement réalisable (B).

\section{A : UNE REVENDICATION JURIDIQUEMENT FONDEE...}

Si l'on se place du strict point de vue des règles du droit international public -particulièrement celle de légalité des États- la revendication par les petits États de la suppression du droit de véto et du statut de membres permanents semble se justifier.

Le droit de véto et le statut de membres permanents sont contraires à l'égalité juridique des États en droit international. Ils relèvent des rapports de force entre les États membres des organisations internationales. ${ }^{551}$ Le Professeur CHEMILLIER-GENDREAU soutient qu' " il faut contester la puissance comme critère de désignation des responsables. Toute l'histoire de la démocratie a consisté à lutter contre la confiscation du pouvoir par les plus riches ou les plus forts [...] Le droit de véto et le statut de membres permanents restent des positions de pouvoirs sans aucune justification». ${ }^{52}$

Le droit de veto constitue pour le Professeur Paul TAVERNIER « une institution rétrograde, vestige d'un passé révolu, qu'il faut abolir, et au mieux comme l'expression d'un mal nécessaire, dont il convient de limiter les effets et corriger les abus $»^{553}$. Toutefois, la limitation des effets et des abus du droit de veto qu'il évoque est très discutable. Dans le débat sur le droit de veto, la plupart des États qui dénoncent son abus ne suggèrent pas son abolition pure et simple ; ils proposent seulement un aménagement de celui-ci. ${ }^{554}$ Les propositions visant à limiter le droit de véto s'articulent autour de deux axes principaux : la limitation du champ d’application de la règle

551 YAO-N’DRE (Paul), Droit des organisations internationales, op.cit., p.28.

552 CHEMILLIER-GENDREAU (Monique), « Pour une organisation de la communauté mondiale », op.cit.p.22.

553 TAVERNIER (Paul), "Commentaire de larticle 27 de la Charte des Nations Unies ", in COT (Jean-Pierre), PELLET (Alain), La Charte des Nations Unies commentaire article par article, op.cit., p.496. Ibid, p.511. 
de l'unanimité et la réduction de sa portée. ${ }^{555}$ Or, serait-il plus démocratique de le supprimer et les États se trouveraient dans une égalité aussi superbe que théorique. ${ }^{556}$

Ce droit que les grandes puissances se sont arrogées dans les organes restreints comporte nécessairement des obligations envers la communauté internationale. Ces privilèges fonctionnels devraient leur permettre d'agir dans le sens des buts définis par les actes constitutifs des organisations internationales. Pourtant, souligne encore le Professeur CHEMILLIER GENDREAU, « ce que les bénéficiaires de ce pouvoir ont fait en un demi-siècle démontre clairement qu'il faut en finir avec ce système. Leur impunité, la consolidation de leur puissance, la militarisation à laquelle ils ont conduit le monde plaident sans hésitation pour une remise en cause de leurs privilèges. $»^{557}$

La suppression du droit de véto et du statut de membres permanents doit être mise en œuvre en vue de l'instauration d'un ordre juridique plus juste dans les relations internationales. Cependant dans la réalité, cette revendication des petits États paraît difficile à réaliser.

\section{B : MAIS DIFFICILE A REALISER}

Le problème de la suppression du droit de véto et du statut de membres permanents des grandes puissances soulève des questions qui relèvent des fondements théoriques des relations internationales. Il faut entendre par les fondements théoriques des relations internationales, « les diverses approches, courants de pensée en matière des relations internationales. Ce sont les représentations mises en forme selon des schémas qui se veulent cohérents et systématiques et qui visent à expliquer les relations internationales, à exposer la structure de la société internationale et à mettre à jour les facteurs déterminants de lévolution des relations internationales. $»^{558}$

Il faut le rappeler, deux grandes théories fondent les relations internationales. Il y a d'un côté les théories réalistes qui prônent que les relations internationales constituent un champ de lutte pour le pouvoir, un ensemble d'activités dans lesquelles règnent la force et la violence. ${ }^{559}$ Les Professeurs GOLDSMITH et POSNER affirment à ce sujet que " realist believe that nations act instrumentally to further their own interests, and they view international behavior largely as a function of distribution of national power [...] the realists think nations are motivated by power, not moral and legal precepts, and thus do not believe that moral and legal rhetoric have significant behavioral consequences on the international stage...» ${ }^{560}$

FLEURENCE (Olivier), La réforme du Conseil de sécurité, létat du débat depuis la fin de la guerre froide, op. cit., p.99.

556 ALBALA (Nuri), " Maudit droit de veto? », in Le monde diplomatique, septembre 2005, p.23.

557 CHEMILIER-GENDREAU (Monique), " Pour une organisation de la communauté mondiale », op.cit., p.22.

558 MELEDJE DJEDJRO (Francisco), Relations internationales, les éditions ABC, Abidjan, janvier 2O10, p.28.

559 Ibid, p.29.

560 GOLDSMITH (Jack L.) and POSNER (Eric A.), « Moral and legal rhetoric in international relations: a rational choice perspective », John M. Olin law and economics working paper, ${ }^{\circ} 108,2^{\text {nd }}$ series, p.4 and 5 . 
Il faut ensuite mentionner les théories idéalistes qui considèrent que les relations internationales sont marquées par la coopération et l'interdépendance des États. ${ }^{561}$ Le Professeur GUZMAN range dans cette catégorie les théories institutionnalistes. Il souligne que « the second category of international relations theories is that of institutionnalists [...] Institutionnalists believe that international cooperation is possible, and that international institutions can play a role in facilating that cooperation... ${ }^{562}$

A priori, la création des organisations internationales par les États correspond à une vision idéaliste des relations internationales. Les États décident de se mettre ensemble dans le cadre des organisations internationales en vue de résoudre les difficultés qui se présentent à eux. Mais cette vision, si juste soit-elle ne doit pas faire illusion.

Dans le fonctionnement interne des organisations internationales, la conception réaliste des relations internationales prend le dessus. Au sein des organes restreints des organisations internationales, les rapports de force s'expriment à travers les privilèges fonctionnels reconnus aux grandes puissances. Le droit de véto et le statut de membre permanent répondent à une vision réaliste des relations internationales. Ils ont été institués en vue d'accorder aux grandes puissances certaines prérogatives fonctionnelles pour tenir compte des responsabilités accrues qui leur incombent et du pouvoir réel dont elles disposent. ${ }^{563}$

La revendication de la suppression de ces deux privilèges fonctionnels s'avère difficile à réaliser car ils sont liés intimement liés à la contribution matérielle des grandes puissances au fonctionnement des organisations internationales. En fait, soutient le Professeur SOUBEYROL, " les grands États ne pourront accepter que leur force industrielle, militaire, financière soit utilisée contre leur gré par une majorité d'États qui n’ont pas grand-chose à perdre dans une action internationale $»^{564}$. Les États qui revendiquent la suppression du droit de véto et le statut de membres permanents ne font même pas partie des contributeurs importants des organisations internationales. La présence de membres permanents au sein du Conseil de sécurité de l'ONU garantit par ailleurs à l'organisation les moyens de son action. Ils représentent le support politique et financier, sans lequel aucune action ne peut être engagée. ${ }^{565}$

Toutefois, il est vrai que si les grandes puissances acceptaient la suppression du droit de veto et le statut de membre permanent, ils bénéficieraient encore de ce quon a pu appeler « le veto financier» ainsi que du « veto administratif». Ils pourraient, en effet continuer à influencer

\footnotetext{
$561 \quad$ Ibid, p.34 à 38 .

562 GUZMAN (Andrew T.), «International law : a compliance based theory », UC Berkeley public law and legal theory working paper series, ${ }^{\circ} 47$, April, 2001, p.15 and 16.

563 BOUTROS-GHALI (Boutros), «Le principe dégalité des États et les organisations internationales », op.cit., p.30.

564 SOUBEYROL (Jacques), « Considérations sur la crise financière de l'ONU », op.cit., p.699.

565 FLEURENCE (Olivier), La réforme du Conseil de sécurité, létat du débat depuis la fin de la guerre froide, op. cit., p.90.
} 
les décisions de l'organisation dans la mesure où ils financent une grande part de son budget et également grâce à la présence de nombreux nationaux aux postes-clés du Secrétariat. ${ }^{566}$

Conscients des difficultés inhérentes à l'hégémonie des grandes puissances dans les organisations internationales, les fondateurs de l'UA se sont orientés dans une logique de rupture en supprimant le droit de véto et le statut de membre permanent au Conseil de paix et de sécurité.

\section{PARAGRAPHE II : LA LOGIQUE DE RUPTURE DU CONSEIL DE PAIX ET DE SECURITE DE L'UA}

Le Conseil de paix et de sécurité de l'UA tire son originalité de la volonté de ses fondateurs de rompre avec le système du Conseil de sécurité de l'ONU. Le Conseil de sécurité a été construit autour d'une logique de responsabilité et de capacité, et non de représentativité. ${ }^{567}$ C'est cet aspect de son fonctionnement que les fondateurs de l'UA nont pas voulu reproduire. La logique de rupture des créateurs du Conseil de paix et de sécurité de l'UA s'exprime par l'inexistence du droit de véto et du statut de membres permanents dans son fonctionnement (A). Mais, cette innovation a sûrement des conséquences sur l'efficacité de l'UA (B).

\section{A : L'INEXISTENCE DU DROIT DE VETO ET DU STATUT DE MEMBRES PERMANENTS}

Assurément, les fondateurs du Conseil de paix et de sécurité de l'UA ont opté pour une égalité juridique entre les membres de cet organe restreint. Cette option apparaît à l'article 5 paragraphe 1 du Protocole relatif à la création de cet organe en ces termes : « Le Conseil de paix et de sécurité est composé de quinze membres ayant des droits égaux ... $»^{568}$

Ce choix s'explique par une volonté persistante des États africains de préserver au maximum leur souveraineté. ${ }^{569}$ Elle se traduit au niveau de l'Acte constitutif de l'UA par la consécration d’objectifs et de principes souverainistes : $:^{570}$

- L' égalité souveraine et l'indépendance de tous les États membres de l'Union ;

- Le respect des frontières existant au moment de l'accession à l'indépendance ;

- La non- ingérence dans les affaires intérieures d’un État membre.

Dans les faits, l'égalité de droits des membres du Conseil de paix se traduit par l'absence de droit de véto et du statut de membres permanents reconnus à certains États. ${ }^{571}$

TAVERNIER (Paul), «Commentaire de l'article 27 de la Charte des Nations Unies », op.cit., p.514. NOVOSSELOF (Alexandra), «L'ONU ou la réforme perpétuelle », op.cit., p.542.

Voir l'article 5 paragraphe 1 du Protocole relative à la création du Conseil de paix et de sécurité de l'UA. AHADZI (Koffi), « réflexions critiques sur l'Union africaine », op.cit, p.92. Ibid.

DUJARDIN (Stéphanie), « l'Union Africaine: objectifs et moyens de gestions des crises politiques et des conflits armés », op.cit., p.69. 
L’exclusion du droit de véto aurait été guidée par deux considérations : le risque constitué par la manipulation de ce droit par certains États à leurs propres fins et la particularité des conflits du continent, c'est-à-dire, la tendance de certains États africains à s'impliquer dans les situations conflictuelles de leurs voisins, voire leur complicité dans leur maintien ou leur aggravation. ${ }^{572}$

Labsence de statut de membres permanents au sein du Conseil de paix et de sécurité serait imputable à l'opposition vigoureuse des États de petite taille qui craignaient, en acceptant le statut de membres permanents, de cautionner loctroi de pouvoirs exorbitants à certains États. ${ }^{573}$

Toutefois, il convient de nuancer ces propos. Le Protocole relatif à la création du Conseil de paix et de sécurité aménage une permanence relative de certains membres dans la mesure où le Conseil est composé de 10 membres élus pour un mandat de deux ans et de 5 membres élus pour un mandat de trois ans dans le but d'assurer la continuité. ${ }^{574}$ Cependant, il est possible que cette continuité se transforme en permanence à travers le jeu des réélections automatiques prévues à l'article 5 paragraphe 3 du Protocole. ${ }^{575}$

La suppression du droit de véto et du statut de membres permanents au sein du Conseil de paix et de sécurité semble se justifier par la volonté des États africains d'éviter de reproduire ce qu'ils considèrent comme une injustice au sein du Conseil de sécurité de l'ONU et qu'ils condamnent énergiquement dans le cadre de la reforme des Nations Unies. ${ }^{576}$ Mais, cette innovation apportée par le Conseil de paix et de sécurité s'avère-t-elle en réalité efficace ? En d’autres termes, cette innovation contribue-t-elle à l'efficacité de l'organisation?

\section{B : LES CONSEQUENCES SUR LE FONCTIONNEMENT DU CONSEIL DE PAIX ET DE SECURITE}

Labsence du droit de véto et du statut de membres permanents a nécessairement des conséquences sur le fonctionnement du Conseil de paix et de sécurité. Mieux, elle devrait permettre un meilleur fonctionnement de l'organisation.

La suppression de ces privilèges fonctionnels traduit la volonté des États africains de faire prévaloir la solidarité régionale en évitant de donner trop de puissances à certains États et en permettant la rotation des sièges. ${ }^{577}$ Avant tout, l'UA est une organisation régionale qui vise à réaliser la solidarité et l'union entre ses membres. Dans le préambule de l'Acte constitutif de cette organisation, les Chefs d'État et de gouvernement, réunis en sommet à Lomé en juillet 2000, proclament qu'ils sont inspirés par les nobles idéaux qui ont guidé les pères fondateurs de l'OUA

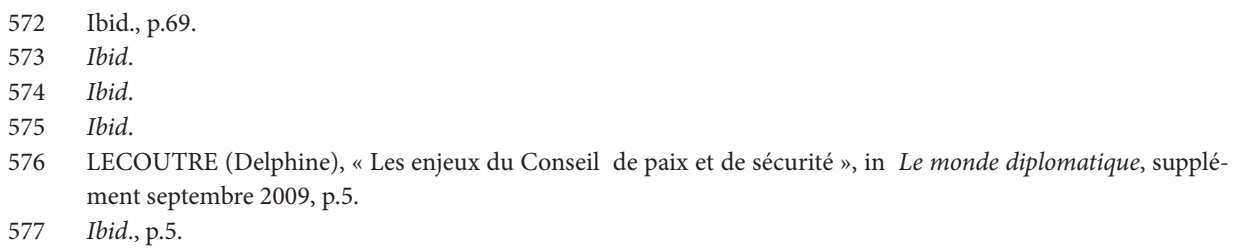


et des générations de panafricanistes dans leur détermination à promouvoir l'unité, la solidarité, la cohésion et la coopération entre les peuples d’Afrique et les États africains. ${ }^{578}$

Loctroi d'un droit de véto et d'un statut de membres permanents serait totalement contraire à cet idéal de solidarité. Pour atteindre les objectifs fixés par le Protocole relatif à la création du Conseil de paix et de sécurité, c'est-à-dire la prévention et la gestion des conflits, il faut éviter de reproduire le schéma de fonctionnement du Conseil de sécurité de l'ONU.

Si l'UA s'est orientée dans le sens d'une égalité entre les États membres au Conseil de paix et de sécurité, évidemment ce choix a des incidences sur son fonctionnement. En vertu de cette égalité, les États doivent contribuer équitablement au fonctionnement de lorganisation. Les États membres doivent prendre toutes les mesures nécessaires pour s'acquitter de leurs contributions au fonctionnement de l'organisation. En outre, cette contribution doit être fixée sur une base égalitaire.

Dans le cas contraire, le Conseil de paix et de sécurité pourrait s’appuyer sur les grands États africains capables de mener des opérations de maintien de la paix de façon efficace. ${ }^{579}$ L'Egypte possède une armée puissante et disponible ; et le Nigéria dispose d'atouts objectivement considérables. ${ }^{580}$ Quant à l'Afrique du Sud, elle dispose d'énormes moyens. Ce pays représente $40 \%$ des richesses de toute l'Afrique subsaharienne et est le dixième contributeur aux opérations de maintien de la paix de l'ONU et le premier contributeur africain avec 3300 hommes. ${ }^{581}$

$\mathrm{Si}$, le Conseil de paix et de sécurité devrait s’appuyer sur ces grands États africains, cela n'impliquerait-il pas que les États membres de l'organisation leur accordent des privilèges fonctionnels tels que le droit de véto et le statut de membres permanents?

578 AHADZI (Koffi), « Réflexions critiques sur l'Union africaine », op.cit., p.86.

579 MVELLE (Guy), L'Union Africaine, fondements, organes programmes et actions, op. cit., p.241.

580 Ibid.

581 Ibid. 


\section{CONCLUSION DE LA PREMIERE PARTIE}

Les fondements de la création d'un organe restreint dans une organisation internationale se résument généralement à deux points essentiels. Il s'agit de rechercher l'efficacité de l'organisation et de l'hégémonie. A côté des «organes intermittents ${ }^{582}$ que sont les organes pléniers, les organes restreints se présentent comme des " organes permanents» ${ }^{583}$ assurant le fonctionnement régulier des organisations internationales. Pour les grandes puissances, les organes restreints permettent de corriger la rigueur du principe d'égalité des États qui prévaut dans les organes pléniers. Les organes restreints demeurent ceux dans lesquelles s'instaurent l'hégémonie des grandes puissances dans les organisations internationales. Contrairement aux organes pléniers qui fonctionnent sous le sceau de l'égalité entre les États membres, les organes restreints sont très souvent marqués par les rapports de force entre les États membres.

Les États créent les organisations internationales dans un but essentiellement fonctionnel. A cet effet, la structure organique des organisations internationales doit nécessairement répondre à cet objectif poursuivi par les États. Dans cette perspective, les organes restreints sont institués en vue de la quête de l'efficacité des organisations internationales. Cette recherche de l'efficacité passe par le caractère permanent des organes restreints et aussi par la contribution à la rationalisation des activités de l'organisation. A ce niveau, les organes restreints jouent un grand rôle dans le bon fonctionnement des organisations internationales.

Mais, ce rôle est fortement entravé par l'inégalité consacrée par les Chartes constitutives entre les États au sein des organes restreints. Celle-ci se traduit par le statut de membres permanents et les droits de vote spéciaux reconnus aux grandes puissances. Ces privilèges fonctionnels qui traduisent les rapports de forces entre les États dans les organisations internationales, freinent l'efficacité de ces organes. Aussi, font-elles l'objet de contestation récurrente de la part des petits États. La réforme du Conseil de sécurité a fait l’objet de nombreuses discussions entre les États. Le document final du sommet de 2005 soulignait que la réforme du Conseil de sécurité était « un élément central de la réforme générale de lorganisation » et le Secrétaire général rappel dans son rapport sur l'activité de l'organisation « qu'il a toujours soutenu que nulle réforme de l'organisation ne serait complète si le Conseil de sécurité en était exclu » et qu'il importait qu'il soit plus représentatif des réalités géopolitiques d’aujourd'hui et que ses méthodes de travail deviennent plus efficaces et transparentes $»{ }^{584}$ Malheureusement, aucun progrès n’a été accompli sur la question. ${ }^{585}$

582 KAMTO (Maurice), « Les mutations institutionnelles de l'OUA », in KAMTO (Maurice), PONDI (Jean-Emmanuel), ZANG (Laurent), L'OUA : rétrospective et perspectives africaines, Economica, Paris, 1990, p.28.

583 Ibid.

584 TAVERNIER (Paul), « Année des Nations Unies, Problèmes juridiques », AFDI, Volume 52, 2006, p.287.

585 Ibid. 


\section{DEUXIEME PARTIE}

\section{L'ORDONNANCEMENT DES POUVOIRS DES ORGANES RESTREINTS}

Le «pouvoir » peut se définir comme l’autorité reconnue aux organes d'une organisation internationale pour accomplir certains actes ou pour remplir certaines fonctions. Les organes restreints sont dotés de pouvoirs définis et organisés dans les limites du traité constitutif des organisations internationales. ${ }^{586}$ Le mot ordonnancement peut s'entendre comme la manière dont sont organisés les pouvoirs des organes restreints.

L'analyse des actes constitutifs des organisations internationales et de la pratique des États permet de déterminer ces pouvoirs (Titre I). Cependant, ils comportent des limites assez considérables (Titre II).

586 Les pouvoirs des organes restreints ne dépassent pas évidemment le cadre constitutionnel tracé par l'Acte constitutif. Cependant, en vertu du principe des compétences implicites des organisations internationales, les organes restreints peuvent exercer des pouvoirs non expressément prévus par la Charte constitutive. La CIJ, dans son célèbre avis du 11 avril 1949 sur la réparation des dommages subis au service des Nations Unies consacre le principe des pouvoirs implicites des organisations internationales. 


\section{TITRE I : LA DETERMINATION DES POUVOIRS DES ORGANES RESTREINTS}

Déterminer les pouvoirs des organes restreints revient à élucider leurs contenus. De ce point de vue, les pouvoirs reconnus aux organes restreints sont des pouvoirs politiques (Chapitre I) et juridiques (Chapitre II). 


\section{CHAPITRE I : LES POUVOIRS POLITIQUES DES ORGANES RESTREINTS}

La notion de pouvoirs politiques évoque les compétences reconnues aux organes restreints en vue d'agir dans la sphère des relations internationales.

Deux points essentiels seront abordés ici. Nous analyserons le problème de la répartition des pouvoirs politiques entre les organes restreints et les organes pléniers (Section I) et nous éluciderons la régulation des relations internationales par les organes restreints (Section II). 


\section{SECTION I : LA REPARTITION DES POUVOIRS POLITIQUES ENTRE LES ORGANES RESTREINTS ET LES ORGANES PLENIERS.}

Dès l'instant qu'il existe des organes à vocation politique dans une organisation internationale, il se pose incontestablement le problème de la répartition des pouvoirs politiques entre ceux-ci.

Comme le fait observer le Professeur Charles CHAUMONT, "léquilibre juridique dans une organisation aussi complexe que l'organisation des Nations Unies exprime une certaine situation politique. ${ }^{587} \mathrm{Ce}$ point de vue peut être aisément ramené aux organisations internationales autres que les Nations Unies. La répartition des pouvoirs politiques entre les organes restreints et les organes pléniers révèle une compétition pour le contrôle des pouvoirs politiques des organisations internationales (Paragraphe I). Elle a nécessairement des répercussions sur la configuration des pouvoirs politiques entre ces deux organes (Paragraphe II).

\section{PARAGRAPHE I : LA COMPETITION POUR LE CONTRÔLE DU POUVOIR POLITIQUE ENTRE LES ORGANES PLENIERS ET LES ORGANES RESTREINTS}

$\mathrm{Au}$ sein des organisations internationales, la question de la répartition des pouvoirs politiques se pose entre les organes pléniers et les organes restreints. Elle témoigne des rapports de force entre la majorité numérique constituée par l'ensemble des États regroupés dans les organes pléniers et la minorité hégémonique représentée par les États membres des organes restreints.

La compétition pour le contrôle du pouvoir politique entre les deux organes s'exprime de deux manières. S'il est vrai que les petits États souhaitent le contrôle des pouvoirs politiques par les organes pléniers $(A)$, en revanche les grandes puissances expriment une volonté de récupération de ces pouvoirs dans les organes restreints (B).

\section{A : LA REVENDICATION DU POUVOIR POLITIQUE PAR LES ORGANES PLENIERS}

Les petits États revendiquent l’exercice du pouvoir politique par les organes pléniers. Cette revendication se fonde sur la majorité numérique de ces États dans ces organes.

Pour le Professeur Michel VIRALLY, cette position s'explique par le fait que les organes pléniers « permettent à tous les États membres de participer à la prise de décision, ce qui est évidemment exclu dans les organes restreints, où l'influence des minorités risque de se faire davantage sentir. $»^{588}$ Le Professeur René-Jean DUPUY fait remarquer aussi que « les principes de la démocratie politique et de la démocratie économique vont s’affronter dans une tension dialectique

587 CHAUMONT (Charles), «L'équilibre des organes politiques des Nations Unies et la crise de lorganisation », op.cit., p.429.

588 VIRALLY (Michel), L’organisation mondiale, Armand Colin, op.cit., p.57. 
que les États situés entretiennent par ailleurs assez volontiers dans le cadre des organisations internationales.» ${ }^{589} \mathrm{La}$ démocratie politique traduit le monopole du pouvoir politique par le plus grand nombre. En revanche, la démocratie économique est celle qui prône le pouvoir de la minorité disposant des moyens matériels les plus importants dans les organisations internationales.

Pour illustrer cette tension entre les organes pléniers et restreints, l'exemple de l'Autorité des fonds marins semble être approprié. Les négociations sur la structure de l'Autorité des fonds marins témoignent de l'ampleur de cette lutte entre les organes pléniers et les organes restreints. C’est un fait, les pays en développement sont largement majoritaires au sein de l’Assemblée générale de cette organisation internationale. ${ }^{590} \mathrm{Il}$ paraît normal qu'ils souhaitent voir affirmer la suprématie politique de l'Assemblée sur le Conseil. ${ }^{591}$

Organe suprême de l'Autorité, l'Assemblée générale pourrait détenir un pouvoir hiérarchique supérieur qui lui permettrait de déterminer et de définir la politique générale de l’organisation. ${ }^{592}$ La conséquence en serait forcément la subordination du Conseil, qui ne constituerait qu'un simple organe d'exécution auquel serait refusé le pouvoir de prendre des décisions à caractère politique. ${ }^{593}$ Cette volonté des petits États siégeant dans les organes pléniers a été exprimée par leurs différentes délégations lors des débats portant sur les rapports politiques entre l'Assemblée générale de l'Autorité des fonds marins et le Conseil.

Monsieur RAKOTOSIHANAKA de la délégation malgache, n’a-t-il pas déclaré que « l'organe suprême de l'Autorité, l'Assemblée, comprenant tous les États membres de l'Autorité devrait être titulaire de tous les pouvoirs politiques de décisions [...] ?». Et n’a-t-il pas rejeté «l'idée de certains États tendant à faire d'un organe restreint, le Conseil, le rouage centrale de l'Autorité, car cela reviendrait à perpétuer le système de véto des grandes puissances... ${ }^{594}$ Dans le même sens, le délégué du Koweï, Monsieur Al IBRAHIM, n’a-t-il pas indiqué que l’Assemblée devrait être «l'organe législatif principal et l’organe suprême de formulation des politiques alors que le Conseil serait l'organe exécutif qui agirait sous le contrôle et selon les directives de l'Assemblée ? ^595

Assurément, les petits États faisant prévaloir la loi du nombre exige l’exercice de la totalité du pouvoir politique par l'Assemblée générale de l'Autorité des fonds marins. Mais, cette exigence qui s'appuie exclusivement sur le nombre semble occulter le pouvoir des contributions financières et économiques des grandes puissances. Or, ce pouvoir économique des grandes puissances conditionne le fonctionnement des organisations internationales. C'est pourquoi, affirme le juge

589 DUPUY (René-Jean), « L'organisation internationale et l'expression de la volonté générale », op.cit., p.540-541.

590 AUDOIN (Jean-Pierre), L'Autorité internationale des fonds marins (D’après les travaux de la troisième Conférence des Nations Unies sur le droit de la mer), op.cit., p.170.

Ibid.

592 Ibid.

593 Ibid.

594 AUDOIN (Jean-Pierre), L’Autorité des fonds marins (D'après les travaux de la troisième Conférence des Nations Unies sur le droit de la mer), op.cit., p.170. Ibid. 
BEDJAOUI, « ceux qui parlent de la puissance de la majorité automatique devrait nuancer quelque peu leur jugement, car il faut se garder de confondre jouissance et exercice d'un droit. Une grande marge, dont la mesure est précisément le sous-développement, sépare la jouissance théorique d'un droit- celui que les États du tiers monde tirent légitimement de leur nombre- et l'exercice effectif de ce droit [...] Et, lorsque l'outil, c'est-à-dire lorganisation, est disponible pour un faire valoir de ce droit, c'est l'artisan, c'est-à-dire le pays en développement, qui se révèle indisponible pour le plein exercice de ce droit. $»^{596}$ La puissance du nombre ne peut pas à elle seule déterminer l'exercice du pouvoir politique par les petits États. Il faut nécessairement tenir compte de la contribution des grandes puissances.Ce constat explique la volonté de récupération des pouvoirs politiques par les organes restreints.

\section{B : LA VOLONTE DE RECUPERATION DES POUVOIRS POLITIQUES PAR LES ORGANES RESTREINTS}

Conscients du poids du nombre des petits États dans les organes pléniers, les grandes puissances essayeront d'inverser les tendances en récupérant les pouvoirs politiques de l'organisation internationale dans les organes restreints. C’est cette approche pragmatique et réaliste qui a été retenue par la Convention de Montégo Bay.

Les pays industrialisés intéressés à l'exploitation des richesses sous-marines ne pourront jamais admettre la suprématie de l'Assemblée générale dont les décisions seront dictées par une majorité d'États peu concernés par l'exploitation industrielle des fonds marins. ${ }^{597}$ La Convention de Montégo Bay de 1982 sur le droit de la mer réalise un compromis à cette épineuse question. ${ }^{598}$ Elle reconnaît que l'Assemblée est « l'organe suprême» de l'Autorité devant lequel les autres organes sont responsables. Le caractère suprême d'un organe se définit non pas tant par les qualificatifs qui lui sont accolés que par la nature et l'ampleur des fonctions dont il est chargé et par ses relations avec les autres organes de l'institution. ${ }^{599}$ Dans le cas de l'Assemblée son autorité se manifeste par son

596 BEDJAOUI (Mohamed), cité par le Professeur DJIENA WEMBOU dans son ouvrage intitulé, Le droit international dans un monde en mutation, essais écrits au fil des ans, à la page 197.

597 AUDOIN (Jean-Pierre), L'Autorité des fonds marins (D'après les travaux de la troisième conférence des Nations Unies sur le droit de la mer), op.cit., p.170.

598 La Convention de Montégo Bay a été ouverte à la signature le 10 décembre 1982 et est le fruit de quatorze années d'efforts déployés tant au niveau du Comité des fonds marins qu'au sein de la Conférence des Nations Unies sur le droit de la mer par près de 150 États représentant les divers systèmes juridiques du monde et appartenant aussi bien à des blocs idéologiques opposés qu’à des niveaux de développement différents. Cette Charte de la mer, qui a pour objet de réglementer les utilisations de la mer, de préciser les droits que les différents groupes d'États peuvent exercer dans les diverses zones maritimes depuis les questions de délimitation jusquà celles de la protection et de la préservation du milieu marin en passant par l'accès aux mers, la navigation, l'exploitation et la conservation des ressources biologiques, la recherche scientifique, l'exploitation minière des fonds marins, la technologie et le règlement des différends .(Voir DJIENA WEMBOU, « L'Afrique et le droit international de la mer ", in le droit international dans un monde en mutation, essais écrits au fil des ans, op.cit.,p. 137 et 138.) DUPUY (René-Jean), VIGNES (Daniels), Traité du nouveau droit de la mer, op.cit., p.648. 
pouvoir d'énoncer des principes directeurs et de formuler des directives générales concernant la politique que le Conseil devra suivre. ${ }^{600}$ Cette solution a été formalisée à l’article 160 alinéa 1 de cette Convention en ces termes : "L'Assemblée, seul organe composé de tous les membres de l'Autorité est considéré comme lorgane suprême de celle-ci devant lequel les autres organes principaux sont responsables, ainsi qu'il est expressément prévu dans la Convention. L’Assemblée a le pouvoir d'arrêter, en conformité, avec les dispositions pertinentes de la Convention, la politique générale de l'Autorité sur toute question ou sur tout sujet relevant de la compétence de celle-ci. » ${ }^{601}$ Cette disposition confère à l'Assemblée le pouvoir d’orienter l'activité des autres organes de l'Autorité, qui devront se conformer aux directives politiques quelle dicte. ${ }^{62}$ Dans l'exercice du pouvoir d'arrêter la politique générale de l'Autorité, l'Assemblée agit en toute autonomie et est libre de suivre ou de rejeter les recommandations du Conseil. Ce faisant, la Convention semble consacrer un lien de subordination entre l'Assemblée générale et le Conseil. Mais en réalité, il n’en est rien.

Aux termes de l'article 162 alinéa 1 de la Convention de Montégo Bay, il est reconnu au Conseil la compétence de « déterminer les politiques spécifiques.» ${ }^{603}$ Il n’est pas facile de percevoir la différence, si tant est qu'elle existe, entre la politique générale définie par l’Assemblée et les politiques spécifiques que le Conseil est chargé d'arrêter. ${ }^{604}$ Où se termine l'une et où commencent les autres ? ${ }^{605}$ Dans tous les cas les politiques spécifiques se résument au pouvoir de contrôle de l'Entreprise, chargée d’explorer et d'exploiter les fonds marins, d'approbation des plans de travail, de détermination de la politique économique du système et du choix des demandeurs d'autorisations de production. ${ }^{606}$ L'entreprise est l’organe opérationnel de l'Autorité des fonds marins. Elle est le bras séculier de l'Autorité des fonds marins.

Les pouvoirs exercés par l'Assemblée ne sont que des pouvoirs d'orientation générale de la politique de l'Autorité. En revanche, le Conseil qui est l'organe restreint jouit de compétences spécifiques relatives à l'exploitation des fonds marins. Dans la mesure où leurs sociétés seront appelées à participer à l'exploitation des richesses minérales sous-marines dans le cadre d'un régime international, les pays industrialisés ont refusé tout système qui faciliterait la suprématie d'un organe au sein duquel ils ne pourraient participer efficacement à la prise de décision. ${ }^{607}$

La Convention de Montégo Bay a attribué les pouvoirs les plus importants, qui portent sur la gestion matérielle de l’Autorité des fonds marins au Conseil dominé par les grandes puissances. Objectivement, cette option de la Convention de Montégo Bay semble être pleinement justifiée. Les

600 DUPUY (René-Jean), La communauté internationale entre le mythe et l'histoire, op.cit., p.98.

601 Voir l'article 160 alinéa 1 de la Convention de Montégo Bay.

602 DUPUY (René-Jean), VIGNES (Daniel), Traité du nouveau droit de la mer, op.cit., p.649.

603 Voir l’article 162 alinéa 2 de la Convention de Montégo Bay.

604 DUPUY (René-Jean), VIGNES (Daniel), op.cit., p.652.

605 Ibid.

606 Ibid.

607 AUDOIN (Jean-Pierre), Lautorité des fonds marins (D'après les travaux de la troisième conférence des Nations Unies sur le droit de la mer), op.cit., p.176. 
États du tiers monde jouissent en réalité de droits que leur procure leur grand nombre dans l'organe plénier de l'Autorité des fonds marins. Mais, les faits montrent qu'ils sont dépourvus de moyens matériels pouvant leur permettre d'exercer effectivement ces droits. L'exploitation des fonds marins requière des moyens économiques et une grande expertise dont les petits États ne disposent pas. Les parties à la Convention ont fait preuve de réalisme en octroyant les pouvoirs opérationnels de l'Autorité des fonds marins au Conseil.

La lutte entre les petits États et les grandes puissances pour la maîtrise du pouvoir politique de l'Autorité des fonds marins est révélatrice des rapports entre la loi du nombre évoquée par les petits États et l'importance des contributions économiques rappelée par les grandes puissances. Elle exprime aussi la difficulté rencontrée par les fondateurs des organisations internationales pour trouver une configuration acceptable des pouvoirs politiques entre les organes pléniers et les organes restreints.

\section{PARAGRAPHE II : LA CONFIGURATION DES POUVOIRS POLITIQUES ENTRE LES ORGANES RESTREINTS ET LES ORGANES PLENIERS}

La configuration des pouvoirs politiques dans les organisations internationales porte sur leur agencement entre les différents organes prévus par la charte constitutive.

En générale, l’analyse des chartes constitutives des organisations internationales permet dobserver deux schémas possibles. En effet, ces deux organes peuvent entretenir des rapports de hiérarchie (A) ou des rapports déquilibre politique (B).

\section{A : LES RAPPORTS DE HIERARCHIE POLITIQUE ENTRE LES ORGANES RESTREINTS ET LES ORGANES PLENIERS}

Les rapports hiérarchiques créent un lien de subordination entre les organes restreints et les organes pléniers. De ce point de vue, deux cas de figure sont à noter. Ce qui est habituel dans les organisations internationales est la suprématie politique des organes pléniers sur les organes restreints (1). Mais dans des cas assez rares, le contraire peut se présenter. Lorgane restreint se trouve alors dans une situation de suprématie politique sur l'organe plénier (2).

1- La suprématie politique des organes pléniers sur les organes restreints

La suprématie politique des organes pléniers sur les organes restreints implique un lien de subordination de l'organe restreint vis-à-vis de l'organe plénier. Au plan politique, l'organe restreint dépend entièrement de l'organe plénier. 
Dans la plupart des organisations internationales africaines et particulièrement à l'UA, la répartition des pouvoirs politiques entre les organes pléniers et les organes restreints révèle un véritable rapport de suprématie des premiers sur les seconds. ${ }^{608}$ Cette suprématie politique des organes pléniers apparaît formellement dans les dispositions des chartes constitutives des organisations internationales africaines. Larticle 6 de la Charte de l'UA dispose que « la Conférence est l'organe suprême de l'Union " ${ }^{609}$ Cet organe réunit les Chefs d'État et de gouvernement ou leurs représentants. Il détient la plénitude des compétences dévolues à l'Union.

Cette suprématie des organes pléniers sur les organes restreints se traduit par les pouvoirs et attributions considérables qui leur sont reconnus. La définition de la politique générale de lorganisation revient aux termes des dispositions des Chartes constitutives aux organes pléniers. Larticle 9 alinéa 1, paragraphe (a) de l'Acte constitutif de l'UA attribue à la Conférence des Chefs d'État et de gouvernement la responsabilité de « définir les politiques communes de l'Union ». ${ }^{610}$

La suprématie de la Conférence des Chefs d'État et de gouvernement est encore renforcée par l'article 9 de l'Acte constitutif. Aux termes de cette disposition la Conférence des Chefs d'État et de gouvernement a compétence pour « recevoir, examiner, et prendre des décisions sur les rapports et les recommandations des autres organes de l'Union et prendre des décisions à ce sujet. " ${ }^{611}$ Cette situation pourrait s'expliquer par la volonté des Chefs d'État africains d'avoir des pouvoirs étendus sur l’organisation. De ce fait, les décisions finales des autres organes doivent nécessairement avoir leur approbation. Les organes restreints que sont la Commission et le Conseil de paix et de sécurité sont subordonnés à la Conférence des Chefs d'État et de gouvernement. Pour preuve, l'article 20 de l'Acte constitutif de l'UA dispose qu' « il est créé une Commission qui est le Secrétariat de l'Union.» ${ }^{612}$ Le Secrétariat d'une organisation internationale est l'organe auquel est confiée la tâche d'exécuter les décisions et les instructions de l'organe plénier. ${ }^{613} \mathrm{Il}$ est utile de rappeler que la Commission remplace le Secrétariat général de l'OUA dans la nouvelle organisation africaine qu'est l'UA. Quant au Conseil de paix et de sécurité, sa subordination à la Conférence résulte du fait qu'il doit sa création et ses pouvoirs à la volonté des Chefs d'État et de gouvernement.

Du reste, cette situation de subordination des organes restreints aux organes pléniers apparaît aussi à l'OIT. Dans les rapports politiques entre la Conférence générale et le Conseil d'administration, le même constat de suprématie de l'organe plénier sur l'organe restreint peut être établi. Elle transparaît au niveau des pouvoirs reconnus au Conseil. Un certain nombre de fonctions ont été confiées au Conseil par la Conférence et figurent soit dans le Règlement de la

\footnotetext{
608 GONIDEC (Pierre-François), Les organisations internationales africaines, L'Harmattan, Paris, 1987, p.37.

609 Voir l'article 6 de l'Acte constitutif de l'UA.

610 Voir l'article 9 de l'Acte constitutif de l'UA.

611 Voir l'article 9 de l'Acte constitutif de l'UA.

612 Voir l'article 20 de l'Acte constitutif de l'UA.

613 MONACO (Riccardo), «Les principes régissant la structure et le fonctionnement des organisations internationales », op.cit.,p.112.
} 
Conférence soit dans le Règlement financier. ${ }^{614}$ Leur analyse révèle que le Conseil d’administration tient certains de ses pouvoirs de la Conférence générale qui est l’organe suprême de l’OIT. Lanalyse des fonctions reconnues au Conseil d'administration par la Constitution de l'OIT révèle qu'il est un organe administratif. ${ }^{615}$

Les rapports entre l'Assemblée générale des Nations Unies et les organes restreints, notamment le Conseil économique et social obéissent aussi à la même logique politique. Pour comprendre la nature des rapports entre ces deux organes, il est nécessaire de se référer aux dispositions de l'article 60 de la Charte. ${ }^{616} \mathrm{Il}$ ressort de ce texte que la relation du Conseil économique et social à l'Assemblée générale est une relation de subordination. Quoique le Conseil soit lui-même un des organes principaux de l'organisation et soit destiné à se charger de l'essentiel du travail dans les domaines économique et social, il agit toujours sous l'autorité de l'Assemblée. ${ }^{617}$ Il est donc lié dans l'exercice de ses fonctions à l'Assemblée générale. A l'analyse de l'ensemble des résolutions du Conseil, l’on constate une tendance croissante à renvoyer les questions de fond à l'Assemblée générale, soit pour décision définitive, soit même pour lui transmettre simplement un rapport soumis au Conseil par un organe subsidiaire ou par le Secrétariat en proposant, selon une formule consacrée, que l'Assemblée générale tienne compte des vues exprimées au cours du débat au Conseil. ${ }^{618}$

La suprématie politique des organes pléniers sur les organes restreints paraît conforme au le principe d’égalité juridique des États. Lorgane plénier, par son universalité, est le seul organe capable, d'une part d’opérer la confrontation de tous les intérêts et de tous les systèmes, d'autre part dexprimer les grands courants qui peuvent, dans une certaine phase historique, entraîner

614 Voir le recueil de règles applicables au Conseil d’administration du Bureau International du Travail, Genève, février 2006, p.3.

615 Les fonctions administratives reconnues par la Constitution au Conseil d'administration sont :

- Lélection du Directeur général (article 8.1).

- la détermination du lieu de la réunion de la Conférence (article 5).

- La détermination de l'ordre du jour de la Conférence (article 14.1).

- La demande de rapports sur les conventions non ratifiées et les recommandations en vertu de l'article 19.5e) et 7 d) iv) et v)

- déterminer la forme des rapports présentés en vertu de l’article 22.

- Examiner les réclamations (articles 24 et 25).

- Le dépôt d'une plainte contre un membre (article 26.4).

- La transmission des plaintes au gouvernement mis en cause (article 26.2).

- La désignation d'une commission d’enquête (article 26.3).

- faire des recommandations à la Conférence pour assurer l'exécution des conclusions des commissions d'enquêtes (articles 33 et 34).

- La formulation et la soumission à la Conférence de règles pour l'institution d'un tribunal compétent pour l'interprétation des conventions (articles 37.2).

- La formulation des règles pour les Conférences régionales (article 38.2).

616 Voir l'article 60 de la Charte des Nations Unies.

617 LEMOINE (Jacques), "Commentaire de l'article 60 de la Charte des Nations Unies ", in COT (Jean-Pierre), PELLET (Alain), la Charte des Nations Unies, commentaire article par article, $2^{\mathrm{e}}$ édition, op.cit., p. 929-930. LEMOINE (Jacques), «Commentaire de l’article 60 de la Charte des Nations Unies », op.cit., p.931. 
une opinion publique internationale. ${ }^{619}$ Les organes pléniers symbolisent la conscience politique universelle de la communauté internationale. C’est justement ce que traduit l'ex- Chef de l'État libyen dans ses propos au sujet de la réforme du Conseil de sécurité : «La réforme des Nations Unies, c'est donner toutes les prérogatives à l’Assemblée générale car toutes les nations du monde sont représentées à l'Assemblée générale avec un siège permanent. Pourquoi cherchons-nous à occuper des sièges permanents au Conseil de sécurité alors que nous avons des sièges permanents à l’Assemblée générale ? L’Assemblée générale est effectivement les Nations Unies. C'est le parlement universel, c'est l’organe législatif si vous le voulez du monde. Donc, toutes les prérogatives devraient être accordées au parlement mondial et non pas au Conseil de sécurité. » ${ }^{620}$

Le rapport de suprématie politique des organes pléniers sur les organes restreints est plus respectueux de la démocratie prônée dans les organisations internationales. Le pouvoir politique dans les organisations internationales devrait logiquement être détenu par l'organe dans lequel se trouve représenté la majorité des États membres. Cependant dans certains cas -très rares-, on note la suprématie politique d'un organe restreint sur l'organe plénier d'une organisation internationale.

\section{2- La suprématie politique des organes restreints sur les organes pléniers}

Il est rare de rencontrer dans les organisations régionales et universelles des organes restreints qui soient supérieurs au plan politique aux organes pléniers.Cependant aux Nations Unies, les rapports du Conseil de sécurité et de l’Assemblée générale en matière du maintien de la paix se présentent comme une exception.

Larticle 24 de la Charte fait du Conseil de sécurité la pierre angulaire du système de sécurité collective établie par la Charte en lui confiant la responsabilité principale du maintien de la paix et de la sécurité internationales. Cet organe devient ainsi le gendarme qui a la charge de concevoir et d'imposer l'ordre mondial. ${ }^{621}$ Nous sommes en présence de l'une des rares hypothèses

619 CHAUMONT (Charles), "Léquilibre des organes politiques des Nations Unies et la crise de l'organisation ", op.cit., p.432.

620 Interview accordée au quotidien ivoirien « Fraternité matin » dans sa parution du samedi 10 et dimanche 11 septembre 2005, $\mathrm{n}^{\circ} 12252$, p.3.

621 DEGNI-SEGUI (René), « Commentaire de l'article 24 paragraphe 1 et 2 de la Charte des Nations Unies », op. cit., p.447. 
dans laquelle l'organe restreint d'une organisation internationale se trouve placé dans une situation de suprématie politique par rapport à l'organe plénier. ${ }^{622}$

Le paragraphe 1 de l'article 24 règle le problème de la distribution des pouvoirs en matière de maintien de la paix et de la sécurité internationales entre les deux organes politiques en établissant un rapport hiérarchique: au Conseil revient la fonction principale et à l'Assemblée générale la fonction résiduelle. ${ }^{623} \mathrm{Il}$ s'agit d'une primauté fonctionnelle du Conseil de sécurité sur l’Assemblée générale, ${ }^{624}$ qui se manifeste concrètement par une primauté procédurale et une primauté matérielle. ${ }^{625}$

Au plan procédural, larticle 12 de la Charte donne un droit de priorité au Conseil de sécurité. Cette disposition fait obligation à l'Assemblée générale de s'abstenir de faire des recommandations sur une affaire dont est saisie le Conseil de sécurité. ${ }^{626}$ Au plan matériel, la primauté du Conseil de sécurité se concrétise par le double monopole de qualification et d'action qui lui est reconnu en cas « de menace contre la paix, d'une rupture de la paix ou d'un acte d'agression.» ${ }^{627}$ Dans le cadre du Chapitre VII de la Charte, le Conseil de sécurité est le seul organe compétent pour constater si un fait constitue une menace contre la paix, une rupture de la paix ou un acte d'agression. En plus, il est encore l'unique organe habilité à prendre des mesures provisoires ou coercitives destinées à juguler une crise internationale.

Dans le dispositif de sécurité collective de la Charte des Nations Unies, le Conseil de sécurité jouit d’une compétence principale au détriment de l'Assemblée générale à qui échoit une responsabilité subsidiaire. ${ }^{628}$ Toutefois, ce système de sécurité collective prévu initialement par la Charte a connu une modification dans la pratique avec la résolution Acheson. Le 3 novembre 1950, l’Assemblée générale adopta la résolution intitulée « union pour le maintien de la paix » ou résolution «Acheson », du nom du Secrétaire d’État américain de l’époque qui présenta le projet.

622 Pour comprendre cette suprématie du Conseil de sécurité des Nations Unies sur l'Assemblée générale, il est important de relever l'intention profonde de certains membres permanents, notamment les États-Unis et l'URSS (devenue par la suite la Russie). A ce sujet, le Professeur Robert CHARVIN souligne que «l'URSS, comme les États-Unis, ne souhaitaient pas le rétablissement du rapport entre l’Assemblée générale et le Conseil de sécurité prévu par la Charte : «le principe d’égalité souveraine des États auquel on se réfère pour appuyer la thèse de la suprématie de l'Assemblée générale sur le Conseil de sécurité, écrit le Professeur TURKIN (Une organisation internationale universelle : illusions et réalités, in comprendre, n³1-32, Venise, 1968), doit être envisagée en tenant compte de la situation réelle dans les relations internationales. Si on confie à l’Assemblée générale, et non au Conseil de sécurité, la responsabilité principale du maintien de la paix [...], cela pourrait entraîner une situation tout à fait dangereuse, avant tout pour les petits États. » (Voir Robert CHARVIN, Relations internationales, droit et mondialisation, un monde en sens unique, op.cit., p.152, précisément la note de bas de page $n^{\circ} 273$ ). DEGNI-SEGUI (René), "Commentaire de l'article 24 paragraphe 1 et 2 de la Charte des Nations Unies », op. cit., p. 448.

$624 \quad$ Ibid, p.449.

625 Ibid.

626 Voir l'article 12 de la Charte des Nations Unies. Cette disposition vise évidemment à assurer la prééminence du Conseil de sécurité sur l’Assemblée générale en matière de maintien de la paix et de la sécurité internationales. Voir le Chapitre VII de la Charte des Nations Unies. CASTENEDA (Jorge), « La valeur juridique des résolutions des Nations Unies », RCADI, 1970- I, p.258. 
En guise de rappel des circonstances historiques de cette résolution, il est important de savoir que de 1911 à 1945, la Corée est sous domination japonaise. Après sa libération en 1948 par les alliés, un gouvernement d'obédience communiste occupe le nord et un autre d'orientation capitaliste est établi en dessous du $38^{\mathrm{e}}$ parallèle. Les coréens du nord envahissent ceux du sud. Le Conseil de sécurité interprète la « politique de la chaise vide » de l'URSS -qui proteste contre la non admission de la Chine populaire à l'ONU- comme une abstention et adopte des résolutions constatant la rupture de la paix. Il invite les États membres à apporter l'aide nécessaire à la Corée du sud pour repousser les assaillants. L’armée américaine, sans avoir reçu mandat de l'ONU, intervient avec des contingents de quelques États contrairement aux dispositions de l'article 42 de la Charte. L'URSS revient au Conseil de sécurité pour s'opposer à de telles mesures en exerçant son droit de véto. C'est ainsi que l’Assemblée générale de l'ONU adopte la résolution 377 (V) le 3 novembre 1950, appelée la résolution Acheson. ${ }^{629}$

Le contenu et l'application de la résolution Acheson se résument essentiellement en trois points. L’Assemblée générale se reconnaît le droit de suppléer le Conseil de sécurité lorsque se présente l'une des trois situations du Chapitre VII et que ce dernier est paralysé par le veto. L’Assemblée générale peut être amenée à préconiser des mesures collectives y compris s'il s’agit d'une rupture de la paix ou d'un acte agression. L’Assemblée générale, si elle ne siège pas, peut être convoquée en session extraordinaire d'urgence, tenue dans les 24 heures, à la demande soit du Conseil de sécurité, soit de l'Assemblée générale. ${ }^{630}$ Cette résolution bouleverse fondamentalement le déséquilibre établi par la Charte des Nations Unies qui accorde une primauté au Conseil de sécurité pour les questions liées au maintien de la paix et de la sécurité internationale. Pour cette raison, la constitutionnalité de cette résolution a été remise en cause. Ainsi, pour le Professeur DEGNI-SEGUI cette « résolution n'est conforme ni à l'esprit ni même à la lettre de la Charte.» ${ }^{631}$ Le Professeur YAO-N'DRE dénonce aussi l'inconstitutionnalité de la résolution Acheson. Cependant, selon lui, «par application de la théorie des compétences implicites la résolution Acheson qui donne compétence à l'Assemblée générale d'intervenir en cas de défaillance du Conseil de Sécurité, n'est pas contraire aux buts assignés à l'ONU. » ${ }^{632}$

La suprématie politique du Conseil de sécurité sur l'Assemblée générale en matière seulement de maintien de la paix et de sécurité internationales demeure l'un des rares cas où un organe restreint d'une organisation internationale se trouve placé dans une position de supériorité politique sur un organe plénier. En dehors de ce domaine, le système général de la Charte soriente dans le sens d'un équilibre entre ces deux organes.

629 YAO-N’DRE (Paul), Droit des organisations internationales, op.cit., p.134.

630 DEGNI-SEGUI (René), "Commentaire de l'article 24 paragraphe 1 et 2 de la Charte des Nations Unies ", op. cit.,p.452 et 453 .

631 DEGNI-SEGUI (René), « Commentaire de l'article 24 paragraphe 1 et 2 de la Charte des Nations Unies », op. cit., p.455.

YAO-N’DRE (Paul), Droit des organisations internationales, op.cit., p.134 et 135. 


\section{B : LES RAPPORTS D'EQUILIBRE POLITIQUE ENTRE LES ORGANES RESTREINTS ET LES ORGANES PLENIERS}

Nous nous intéresserons aux manifestations de léquilibre existant entre les organes restreints et les organes pléniers (1) et ses corollaires (2).

\section{1- Les manifestations de léquilibre politique}

La meilleure garantie de l'équilibre entre les organes d'une organisation internationale résulte du fait qu'ils sont placés par l'acte constitutif sur un plan de parité. ${ }^{633}$ L'équilibre politique qui pourrait exister entre les organes restreints et les organes pléniers découle nécessairement des dispositions des Chartes constitutives des organisations internationales.

Léconomie générale de la Charte de l'ONU montre qu'un équilibre a été mis en place entre l'Assemblée générale et le Conseil de sécurité. La recherche de l'équilibre n’apparaît réellement qu'entre ces deux organes politiques que sont le Conseil de sécurité et l'Assemblée générale. ${ }^{634}$ Des six organes principaux que comptent les Nations Unies, le Conseil de Sécurité et l'Assemblée générale sont les organes politiques les plus importants. C’est en eux que se réalise le type d'équilibre qui caractérise les exigences politiques dominantes de l'ONU. ${ }^{635}$ Léquilibre entre ces deux organes principaux repose, avant tout sur l'institution d'un bicéphalisme, c'est-à-dire de la dualité des organes politiques souverains : le Conseil de sécurité, dominé par les grandes puissances, et l’Assemblée générale, dominée par le nombre, c'est-à-dire la majorité des petits États. ${ }^{636}$

Cependant, comment se manifeste concrètement l'équilibre politique entre l'Assemblée générale et le Conseil de sécurité ? La clé de cet équilibre se trouve dans les articles 11, 14, et $24 .{ }^{637}$ Ces trois dispositions constitutionnelles constituent la pierre angulaire de l'équilibre politique entre le Conseil de sécurité et l'Assemblée générale. Il se réalise tant au niveau des fonctions que des pouvoirs de ces deux organes. Aux termes de l'article 11, "l’Assemblée générale étudie [...] les questions qui se rattachent au maintien de la paix.» ${ }^{638} \mathrm{Et}$, l'article 14 dispose que «...l'Assemblée générale peut recommander les mesures propres à assurer l'ajustement pacifique de toute situation.... $»^{639}$ En ce qui concerne le Conseil de sécurité, l’article 24 dispose qu' « afin d’assurer l'action rapide et efficace de lorganisation, ses membres confèrent au Conseil de sécurité la

633 MONACO (Riccardo), «Les principes régissant la structure et le fonctionnement des organisations internationales », op.cit., p.112.

634 CHAUMONT (Charles), "L'équilibre des organes politiques des Nations Unies et la crise de l'organisation ", op.cit., p.429.

635 Ibid, p.430.

636 VIRALLY (Michel), Lorganisation mondiale, op.cit., p.107.

637 CHAUMONT (Charles), "Léquilibre des organes politiques des Nations Unies et la crise de l'organisation ", op.cit., p.431.

638 Voir l'article 11 de la Charte des Nations Unies.

639 Voir l'article 14 de la Charte des Nations Unies. 
responsabilité principale du maintien de la paix et de la sécurité internationales... " ${ }^{640}$ L’analyse de ces dispositions de la Charte nous situe sur le sens de l'équilibre établi entre le Conseil de sécurité et l'Assemblée générale. L’Assemblée étudie ce qui "se rattache » à la paix, le Conseil « agit » pour assurer la paix. A l'intérieur du même domaine du maintien de la paix, sétablit ainsi une sorte de répartition entre la fonction détude et la fonction d'action. ${ }^{641}$

Léquilibre politique entre le Conseil de sécurité et l'Assemblée générale se vérifie aussi au niveau de la prise de certaines décisions fondamentales. La Charte des Nations Unies réserve certains actes importants pour l'avenir de l'organisation à une décision exigeant l'accord des deux grands organes politiques : l'admission d'un nouveau membre, ${ }^{642}$ la suspension ${ }^{643}$ ou l'exclusion d'un membre, ${ }^{644}$ la nomination du Secrétaire général ${ }^{645}$ résultent d'une décision de l'Assemblée générale prise sur recommandation du Conseil de sécurité $e^{646}$.

Dans ses avis consultatifs du 28 mai 1948 et du 3 mars 1950 sur la compétence del’Assemblée générale pour l'admission d'un État aux Nations Unies, la CIJ s'est prononcée en ce sens. ${ }^{647}$ La Cour a déclaré n’avoir « aucun doute » sur le sens de l’article 4, paragraphe 2 de la Charte : « Celui-ci exige deux choses pour que soit effectuée l'admission : une " recommandation » du Conseil de sécurité et une « décision» de l’Assemblée générale, la recommandation devant, par la nature des choses, précéder la décision [....], la recommandation du Conseil de sécurité est la condition préalable de la décision de l'Assemblée par laquelle se fait l'admission.» ${ }^{68}$ Le Conseil de sécurité jouit d'un véritable pouvoir de codécision ${ }^{649}$ avec l'Assemblée générale dans la procédure d’admission, de suspension et d'exclusion d'un État membre, de même dans la procédure de nomination du Secrétaire général. Ce

640 Voir l'article 24 de la Charte des Nations Unies.

641 CHAUMONT (Charles), "Léquilibre des organes politiques des Nations Unies et la crise de lorganisation », op.cit., p.431.

642 Voir l'article 4 de la Charte des Nations Unies.

643 Voir l'article 5 de la Charte des Nations Unies.

644 Voir l'article 6 de la Charte des Nations Unies.

645 Voir l'article 97 de la Charte des Nations Unies.

646 VIRALLY (Michel), "La valeur juridique des recommandations des organisations internationales ", AFDI, 1956, p.76.

647 Le problème de l'admission fut soulevé à l’occasion de deux avis consultatifs démandés à la CIJ : l'un portant sur les conditions de fond de l'admission, l'autre sur les conditions de procédure. Dans l'avis consultatif du 28 mai 1948, la CIJ fut consultée à propos de la controverse soulevée par Union soviétique qui, durant la période dite de " guerre froide » (1948-1955), entendait subordonner l'admission d'un État à l'admission d'autres États (idéologiquement proches d'elle). La Cour estima que la liste des conditions de fond posées par l'article 4 de la Charte était limitative et que ce marchandage était illégal.

Dans l'avis du 3 mars 1950, l'Union soviétique continuant à paralyser (après l’avis de 1948), l'admission de nouveaux membres, la Cour fut consultée sur le point de savoir si, aux termes de l'article 4 de la Charte, l'Assemblée générale pouvait passer outre à l'absence de recommandation du Conseil de sécurité (une éventuelle recommandation se heutant au véto soviétique). La Cour répondit par la négative, car la recommandation du Conseil de sécurité est le support essentiel de la décision de l’Assemblée générale. (Voir RUZIE (David), Droit international public, $18^{\mathrm{e}}$ édition, Dalloz, 2006, p.217).

648 VIRALLY (Michel), « La valeur juridique des recommandations des organisations internationales », op.cit., p.76. Ibid, p.77. 
pouvoir de codécision du Conseil de sécurité traduit une volonté affichée des grandes puissances, membres permanents de cet organe, de se prononcer et même de poser leur droit veto sur les questions essentielles, fondamentales pour la vie de l'organisation. ${ }^{650}$

Dans le schéma de la Charte, un équilibre politique a été institué entre le Conseil de sécurité et l'Assemblée générale. Quels en sont les corollaires?

\section{2- Les corollaires de l'équilibre politique}

Léquilibre politique entre les organes restreints et les organes pléniers emporte des conséquences considérables tant au plan politique que juridique.

En ce qui a trait à l'aspect politique, la démocratie dans les organisations internationales ne peut se réaliser dans l'hypothèse d'un équilibre entre les organes politiques. ${ }^{651}$ L'équilibre permet d'aboutir à une juste répartition des pouvoirs politiques entre «la masse » représentée par l’ensemble des États membres siégeant dans les organes pléniers et « le petit nombre » constitué par les membres des organes restreints. ${ }^{62}$ Dans la pratique, un tel équilibre évite qu’un seul organe de lorganisation ait le monopole du pouvoir de décision sur les questions importantes.

Léquilibre établi entre le Conseil de sécurité et l’Assemblée générale constitue une garantie pour la démocratie aux Nations Unies en ce sens qu'il institue un système de contre pouvoir politique entre ces deux organes. Comme le fait observer MONTESQUIEU, « c'est une expérience éternelle que tout homme qui a du pouvoir est porté en abuser; il va jusquà ce qu'il trouve des limites [...]. Pour quon ne puisse abuser du pouvoir, il faut que par la disposition des choses, le pouvoir arrête le pouvoir $» .{ }^{653}$ Aux Nations Unies, le fait que certaines fonctions électives de grandes importances soient soumises à une décision conjointe du Conseil de Sécurité et de l'Assemblée générale traduit la volonté de démocratiser la prise de décisions dans ces matières d'intérêt général pour l'ensemble des États-membres.

$\mathrm{Du}$ point de vue juridique, léquilibre politique entre ces deux organes entraîne inéluctablement des conflits de compétence. Ils se produisent dans certaines hypothèses bien précises. Il convient de mentionner la situation dans laquelle un organe dépasse les limites de ses compétences et empiète sur celles d'un autre. ${ }^{654}$ Dans ce cas, l'un des deux organes prend des initiatives dans le domaine constitutionnel de l'autre organe, entraînant par là même un déséquilibre institutionnel. Pour le Professeur Charles CHAUMONT « le déséquilibre a commencé à apparaitre

650 Cependant, il convient de souligner que pour l'élection des juges à la CIJ, la décision de l’Assemblée générale n’est pas ici subordonnée à la décision du Conseil de sécurité. Les deux organes agissant parallèlement et en toute indépendance l'un de l'autre. 
lorsque l'Assemblée générale a supervisé le Conseil jusque dans la fonction propre de celui-ci, qui est l'action concrète. C’est l'essence même de la résolution Acheson du 3 novembre 1950, tout au moins dans la mesure où, dans un cas déterminé, elle permet à l'Assemblée générale de qualifier l'agression et de mettre en mouvement des opérations militaires (deux compétences que la Charte réserve au Conseil dans l'article 39). »655

Avec la résolution Acheson, l'Assemblée générale empiète de façon considérable sur les pouvoirs du Conseil de sécurité dans le domaine du maintien de la paix et de la sécurité internationales. Le recours à cette résolution a des effets négatifs sur le fonctionnement de l'Assemblée générale. Le Professeur Charles CHAUMONT souligne encore que « le processus entamé par la résolution Acheson n’a pas été un processus de destruction du Conseil ; il a été plutôt un processus d'autodestruction de l'Assemblée générale. En s’alourdissant de la fonction du Conseil, l'Assemblée a pris un poids que son armature ne lui permet pas de soutenir. ${ }^{656}$

La répartition des pouvoirs politiques entre les organes restreints et les organes pléniers traduit les rapports de force entre les États membres des organisations internationales. Une fois le point touchant la distribution des pouvoirs réglé, il est important de réfléchir sur létendue des pouvoirs politiques des organes restreints.

\section{SECTION II : L'ETENDU DES POUVOIRS POLITIQUES DES ORGANES RESTREINTS}

Les pouvoirs politiques des organes restreints peuvent être compris comme l'ensemble des compétences reconnues dans la sphère politique par les États membres.

Létendu des pouvoirs politiques des organes restreints dans les relations internationales est très considérable. Ils constituent un ersatz d'un pouvoir exécutif dans l'ordre international (paragraphe I) et contribuent à la régulation des relations entre les États (paragraphe II).

\section{PARAGRAPHE I : LES ORGANES RESTREINTS, UN ERSATZ D'UN GOUVERNEMENT A L'ECHELLE INTERNATIONALE}

Dire que les organes restreints sont un ersatz d'un gouvernement à léchelle internationale revient à les considérer comme un succédané, un organe de remplacement d'un gouvernement à l'échelle internationale.

Dans une société internationale où il n'existe pas d’organe exécutif, les organes restreints des organisations internationales peuvent constituer un ersatz d'un gouvernement à l'échelle internationale. Cette vocation des organes restreints à représenter un pouvoir exécutif (A) se heurte à la souveraineté des États (B).

655 CHAUMONT (Charles), « Léquilibre entre les organes politiques des Nations Unies et la crise de l'organisation », op.cit., p.434.

656 Ibid. 


\section{A : LA VOCATION DES ORGANES RESTREINTS A REPRESENTER UN POUVOIR EXECUTIF A L'ECHELLE INTERNATIONALE}

Dans lordre interne des États, le pouvoir exécutif est celui qui est chargé de mettre en œuvre, d'appliquer les règles adoptées en son sein. Dans cet ordre, les sujets de droit sont placés au-dessous d'un pouvoir qui pose la loi et en impose le respect. ${ }^{657}$ De ce fait, le droit interne devient un droit de subordination qui conditionne des sujets susceptibles dêtre contraints au besoin par la force, grâce à un appareil institutionnel adéquat. ${ }^{658}$

Au contraire, l’ordre international ne comprend que des phénomènes de relations, qui ne supportent aucune autorité supérieure à celle des États juxtaposés. ${ }^{659} \mathrm{Il}$ n'y a donc pas dans l'ordre international un appareil institutionnel chargé d’appliquer le droit. Mais, avec la création des organisations internationales, « on tend à accéder à un système institutionnel s'inspirant d'une conception différente, susceptible de retrouver les caractères du droit interne et d'offrir l'image d'un ensemble doté d’organes compétents pour légiférer, juger et exécuter.» ${ }^{660}$ Dans la pratique, cette évolution se matérialise par les organes mis en place au sein des organisations internationales. Particulièrement, les organes restreints sont investis d'un pouvoir exécutif dans les relations internationales. Les Chartes constitutives des organisations internationales sont assez explicites sur ce point. Elles reconnaissent aux organes restreints un domaine d'action assez important.

L'Acte constitutif de l'UA prévoit une Commission composée d'un Président et d'un Vice-président et de huit Commissaires tous nommés par la Conférence des Chefs d'État et de gouvernement ou leurs représentants. ${ }^{661}$ Les Commissaires ont à leur charge huit portefeuilles : paix et sécurité, affaires politiques, infrastructures et énergie, affaires sociales, ressources humaines, sciences et technologie, commerce et industrie, économie rurale et agriculture, affaires économiques. ${ }^{662}$ Ces différents portefeuilles constituent les domaines dans lesquels la Commission est chargée d'intervenir sur le continent africain.

Aux termes de l'article 6 du Protocole relatif à la création du Conseil de paix et de sécurité, il « assume les fonctions dans les domaines suivants :

a. Promotion de la paix, de la sécurité et de la stabilité en Afrique ;

b. Alerte rapide et diplomatie préventive ;

c. Rétablissement de la paix, y compris les bons offices, la médiation, la conciliation et l'enquête ;

d. Opération d'appui à la paix et intervention...;

657 DUPUY (René-Jean), Le droit international, Collection Que sais-je ?, op.cit., p.5.

658 Ibid.

659 DUPUY (René-Jean), La communauté internationale, entre le mythe et l'histoire, op.cit., p.43.

660 DUPUY (René-Jean), Le droit international, Collection Que sais-je ?, op.cit., p.6.

661 MVELLE (Guy), L'Union africaine, fondements, organes, programmes et actions, op.cit., p.216.

662 Ibid, p.217. 
e. Consolidation de la paix et reconstruction post-conflit,

f. Action humanitaire et gestion des catastrophes... ${ }^{663}$

Dans le cas de l'ONU, l'article 24 alinéa 1 de la Charte fait du Conseil de sécurité l’organe exécutif dans le domaine du maintien de la paix et de la sécurité internationales. Aux termes de cette disposition, les membres de l'organisation « ...confèrent au Conseil de sécurité la responsabilité principale du maintien de la paix et de la sécurité internationales... " ${ }^{664}$ Par l’effet de cette disposition, il devient le seul organe ayant compétence pour agir au nom de tous les États membres dans le domaine du maintien de la paix et de la sécurité internationales. Larticle 62 de la Charte définit le domaine d'action du Conseil économique et social. Il s'agit entre autres des " domaines économique, social, de la culture intellectuelle et de l'éducation, de la santé publique.» ${ }^{665}$ Cet organe est aussi compétent pour « faire des recommandations en vue du respect effectif des droits de l'homme et des libertés fondamentales pour tous... » ${ }^{666}$

$\mathrm{Du}$ reste, avec les organes restreints " la valeur instrumentale ${ }^{667}$ des organisations internationales semble se préciser assez nettement. A l'instar du gouvernement dans l’ordre interne, ses fondateurs disposent d'un instrument pouvant leur permettre d'exécuter leurs missions dans les relations internationales. Mais dans la réalité, cette vocation des organes restreints à incarner un pouvoir exécutif à léchelle internationale semble être remise en cause par les États membres de lorganisation.

\section{B : L'OBSTACLE DECOULANT DE LA SOUVERAINETE DES ÉTATS MEMBRES}

En dépit de son appartenance à une organisation internationale, l'État garde sa souveraineté. ${ }^{668}$ Celle-ci se définit comme le pouvoir suprême en vertu duquel l'État n'est assujetti à aucun autre État dans l’ordre international. Cette définition est partagée par le Professeur GUZMAN lorsqu'il affirme que « sovereign states are not subject to any obligation unless they have consented to it. $"$ "69

La vocation des organes restreints à représenter un gouvernement à léchelle internationale se heurte à cet obstacle majeur que constitue la souveraineté des États. Cette notion centrale du droit international public donne un caractère particulier à la question de l'existence d'un pouvoir exécutif dans l'ordre international. Cet ordre, affirme le Professeur DUPUY «ne supporte aucune

\footnotetext{
663 Voir l'article 6 du Protocole relatif à la création du Conseil de paix et de sécurité de l'UA.

664 Voir l'article 24 de la Charte de l'ONU.

665 Voir l'article 62 alinéa 1 de la Charte de l'ONU.

666 Voir l'article 62 alinéa 2 de la Charte de l'ONU.

667 VIRALLY (Michel), Lorganisation mondiale, op.cit., p.26.

668 DUPUY (René-Jean), La communauté internationale entre le mythe et l'histoire, op.cit., p.62.

669 GUZMAN (T. Andrew), «International law : a compliance based theory », UC Berkeley public law and legal theory working paper, $\mathrm{n}^{\circ} 47$, April 2001, p.8 and 9.
} 
autorité supérieure à celle des États juxtaposés [...] On y chercherait en vain un pouvoir central. » ${ }^{670}$ Dans l'ordre international, le quasi-monopole par les États de la qualité d’agent juridique, ${ }^{671}$ implique l'inexistence d'un pouvoir exécutif au dessus d'eux.

Cette idée est soutenue par Madame Simone BASTID. Selon elle, " en réalité, il n’y a pas dans la vie internationale actuelle de fonctions proprement exécutive appartenant à un organe international, ou du moins cette fonction exécutive n'existe que dans un domaine limité, et plutôt au profit des organes permanents de l'Administration internationale. En général, ce sont les États qui exécutent et non pas un organe quel qu'il soit.» ${ }^{672}$

Dans le même ordre d'idée, les Professeurs GOLDSMITH et LEVINSON affirment que " domestic law is taken to be the paradigm of how a legal system should work. Legal rules are promulgated and updated by a legislature or by common law courts subject lo legislative revision [...] The individuals to whom law is addressed have an obligation to obey law making authorities, even when legal rules stand in the way of their interest or are imposed without their consent [...] International law has no centralized legislature or hierarchical court system authorized to create, revised or specify the application of legal norms... ${ }^{673}$

Même si les organisations internationales, à travers leurs organes restreints adoptent des actes juridiques destinés à s’appliquer aux États, en définitive leur exécution incombe aux États souverains. Dans les faits, elles ont toujours recours aux États pour exécuter les actes émanant de leurs organes restreints. Les mesures coercitives prises par le Conseil de sécurité en vertu de l'article 42 de la Charte peuvent permettent d’expliciter ce point. Aux termes de cette disposition, «si le Conseil de sécurité estime que les mesures prévues à l'article 41 seraient inadéquates ou qu'elles se sont révélées telles, il peut entreprendre, au moyen des forces aériennes, navales ou terrestres, toute action qu'il juge nécessaire au maintien ou au rétablissement de la paix et de la sécurité internationales. Cette action peut comprendre des démonstrations, des mesures de blocus et d'autres opérations exécutées par des forces aériennes, navales ou terrestres de membres des Nations Unies.» ${ }^{674}$ L'impossibilité de recourir à l'article 42, faute de forces aériennes, navales ou terrestres à la disposition du Conseil, a été dénoncée comme un vice fondamental de l'organisation. ${ }^{675}$

De ce fait, il est difficile de prétendre que c'est le Conseil de sécurité, en tant qu'organe exécutif de l'ONU qui met en application directement le contenu de l'article 42 de la Charte. Ce sont les États membres, disposant des moyens militaires qui exécutent les résolutions adoptées sur le fondement de cette disposition. Cette analyse est aussi valable pour le Conseil de paix et de

\footnotetext{
$670 \quad$ Ibid, p.43.

671 Ibid.

672 BASTID (Simone), Cours de droit international public, fascicule III, Les cours de droit, Paris, 1969-1970, p.467.

673 GOLDSMITH (Jack L.) and LEVISON (Daryl J.), «Law for states: international law, constitutional law, public law», havard law school public law and legal theories working paper series, $\mathrm{n}^{\circ} 9-16,2009$, p.1.

674 Voir l'article 42 de la Charte des Nations Unies.

675 FISCHER (Georges), "Commentaire de l'article 42 de la Charte de l'ONU », in COT (Jean-Pierre), PELLET (Alain) La Charte des Nations Unies, commentaire article par article, $2^{\mathrm{e}}$ édition, op.cit., p.716.
} 
sécurité de l'UA. Aux termes de l'article 13 du Protocole relatif à sa création, dans la réalisation de sa mission, cet organe s’appui sur « une force africaine pré positionnée, composée de contingents multidisciplinaires prêts à être déployés et stationnés dans leurs pays d’origine.» Cette disposition montre la dépendance étroite du Conseil de paix et de sécurité vis à vis des États membres. Leurs contributions au fonctionnement de cet organe est considérable. Elles se situent au plan opérationnel par la mise à la disposition des contingents nationaux et par le financement des activités de cet organe.

En dépit des obstacles que constituent les États à leur statut de pouvoir exécutif dans lordre international, les organes restreints participent à la régulation des relations internationales.

\section{PARAGRAPHE II : LA REGULATION DES RELATIONS INTERNATIONALES PAR LES ORGANES RESTREINTS}

Les organes restreints favorisent la régulation des relations internationales. Cette régulation se manifeste surtout à travers les pouvoirs opérationnels des organes restreints (A). Mais, il est important de souligner que ces pouvoirs opérationnels comportent des carences (B).

\section{A : LES POUVOIRS OPERATIONNELS DES ORGANES RESTREINTS}

Les pouvoirs opérationnels sont des pouvoirs d'action qu'exercent les organisations internationales. Il s'agit de compétences autres que celles d'édiction des normes. ${ }^{676}$ Ces pouvoirs sont relatifs à la dimension sécuritaire et structurelle du maintien de la paix dans les relations internationales.

La dimension sécuritaire du maintien de la paix concerne l'absence de recours à la force armée dans les relations internationales. Cette approche du maintien de la paix vise à créer les conditions nécessaires au non recours à la force entre les États, acteurs principaux des relations internationales. Ceux-ci tentent de réglementer le non recours à force en mettent en place un système qui fonctionne autour des organes restreints.

Les rédacteurs de la Charte des Nations Unies ont désigné un organe qui a prioritairement la charge d'assurer cet objectif de maintien de la paix. Larticle 24 alinéa 1 dispose à cet effet qu' « afin d'assurer l'action rapide et efficace de l'organisation, ses membres confèrent au Conseil de sécurité la responsabilité principale du maintien de la paix et de la sécurité internationales et reconnaissent qu'en s'acquittant des devoirs que lui impose cette responsabilité le Conseil agit en leur nom.» ${ }^{677}$

Le Conseil de sécurité a pris des résolutions visant à réguler des problèmes politiques fondamentaux dans les relations internationales comme celui du terrorisme. Dans sa résolution

676 YAO-N'DRE (Paul), Droit des organisations internationales, op.cit., p.110.

677 Voir l'article 24 de la Charte des Nations Unies. 
1373 (2001) adoptée à l'unanimité des États membres, le Conseil décide que « chaque État a le devoir de s'abstenir d’organiser et d'encourager des actes de terrorisme sur le territoire d'un autre État, d'y aider ou d'y participer, ou de tolérer sur son territoire des activités organisées en vue de perpétrer de tels actes.» ${ }^{678}$ Il ne se limite pas seulement à l'édiction d’une simple résolution. En raison de l'importance politique que revêt la question du terrorisme dans les relations internationales, cette résolution 1373 du Conseil de sécurité fait l’objet d'un mécanisme de suivi organisé. Il s'agit d'un comité contre le terrorisme (CCT). ${ }^{679}$ Dans sa mission, le CCT impose aux États de lui rendre compte des mesures prises dans le cadre de la lutte anti-terroriste du fait que l'objet du contrôle opéré par le CCT porte sur la conformité des mesures prises ou la mise en œuvre des exigences posées par le Conseil de sécurité. ${ }^{600}$

Cette résolution 1373 sera suivie de la résolution 1566 (2004) du 8 octobre 2004. Dans cette résolution, le Conseil rappelle dans le paragraphe 3 du dispositif que « les actes criminels, notamment ceux dirigés contre des civils dans l'intention de causer la mort ou des blessures graves [...] qui sont visés et érigés en infractions dans les conventions et protocoles internationaux relatifs au terrorisme ne sauraient en aucune circonstance être justifiés par des motifs de nature politique, philosophique et idéologique ${ }^{681}$ et demande "à tous les États de les réprimer par des sanctions à la mesure de leur gravité.» ${ }^{682}$

A l'instar de la résolution 1373, le Conseil renforce son action par la création d'un groupe de travail plénier devant faire des propositions concrètes dans le but à la fois délargir la liste des personnes ou entités liées à des activités terroristes au-delà de celle prise en application des résolutions visant Al-Quaïda et les Talibans et d'étudier la création d'un fonds international d'indemnisation des victimes d'actes terroristes. ${ }^{683}$ De toute évidence, le Conseil de sécurité des Nations Unies a pris conscience du fait que le terrorisme est devenu un problème de sécurité collective globale qu'il convient de traiter avec une attention soutenue supposant de dégager une méthode efficace et efficiente. ${ }^{684} \mathrm{Il}$ en va de même dans le cadre régional avec le Conseil de paix et de sécurité de l'UA.

Concernant la gestion des crises politiques et la gestion des conflits armés, l'UA en fait une priorité en se fixant pour objectif de promouvoir la paix, la sécurité et la stabilité sur le continent sur la base de principes classiques comme $:^{685}$

\footnotetext{
678 Voir affaires et documents de droit international, Centre de Publication Universitaire, Tunis, 2005, p.310.

679 KOKOROKO (Komla Dodzi), « Réflexion sur le pouvoir législatif du Conseil de sécurité de l'ONU », RBSJA, décembre $2007, \mathrm{~N}^{\circ} 19$, p. 128 .

$680 \quad$ Ibid, p.129.

681 Voir la résolution 1566 du 8 octobre 2004 du Conseil de sécurité des Nations Unies.

682 Ibid.

683 KOKOROKO (Komla Dodzi), « Réflexion sur le pouvoir législatif du Conseil de sécurité de l'ONU », op.cit., p.130 et 131 .

684 Ibid, p.13O.

685 Voir l'article 3, paragraphe g de l'Acte constitutif de l'UA.
} 
- le règlement pacifique des différends entre les États membres de l'Union par des moyens appropriés ; ;86

- L'interdiction de recourir ou de menacer de recourir à l'usage de la force entre les États membres de l'Union ; ${ }^{687}$ Et de principes nouveaux comme :

- Le droit de l'Union d'intervenir dans un État membre sur décision de la Conférence, dans certaines circonstances graves, à savoir les crimes de guerre, le génocide et les crimes contre l'humanité ; 688

- La condamnation et le rejet des changements anti-constitutionnels de gouvernement... etc $^{689}$

Pour atteindre l'objectif de paix et de sécurité sur le continent africain et se conformer aux principes sur lesquels elle entend se fonder, l'UA s’est dotée d'un organe décisionnel et opérationnel de gestion et résolution des conflits armés : le Conseil de paix et de sécurité. ${ }^{690}$

Le cadre d'action du Conseil de paix et de sécurité est très large. Il « anticipe et prévient les différends et les conflits, ainsi que les politiques susceptibles de conduire à un génocide et à des crimes contre l'humanité.» ${ }^{691}$ En outre, il faut mentionner le domaine de l'appui à la paix et de l'intervention. A ce titre, le Conseil de paix et de sécurité s'appuie sur la Commission qui sous son autorité et en consultation avec toutes les parties impliquées dans un conflit, prend toutes les initiatives qu'il juge appropriées pour prévenir, gérer et régler les conflits. ${ }^{692}$

Le Conseil de paix et de sécurité peut recommander à la Conférence l'intervention au nom de l'Union dans un État membre en cas de circonstances graves, à savoir les crimes de guerre,

686 Voir l'article 4, paragraphe e de l'Acte constitutif de l'UA.

687 Voir l'article 4, paragraphe f de l'Acte constitutif de l'UA.

688 Voir l'article 4, paragraphe h de l'Acte constitutif de l'UA.

689 Voir l'article 4, paragraphe p de l'Acte constitutif de l'UA.

690 Il faut préciser que le Conseil de paix et de sécurité n'existait pas lors de l'adoption de l’Acte constitutif de l'UA en juillet 2000. Ce n'est qu’en juillet 2002, en exécution de son mandat, que le Secrétaire général, Amara ESSY, en consultation avec le comité des Ambassadeurs, a présenté au sommet de l'UA un projet de Protocole sur la création d'un Conseil de paix et de sécurité au sein de l'UA. Ainsi, sur recommandation du Conseil des Ministres, la Conférence adopte le Protocole et lance un appel à tous les États membres pour qu'ils le signent et le ratifient en vue de son entrée en vigueur rapide. Le Protocole est entré en vigueur en décembre 2003.

691 Voir l'article 7 paragraphe 1 du Protocole relatif au Conseil de paix et de sécurité de l'UA. Dans le cadre de la prévention des conflits, l'UA s'est dotée de deux nouveaux instruments : le groupe de sages et le système d'alerte rapide. Le groupe des sages est composé de cinq personnalités africaines sélectionnées par le Président de la Commission et nommées pour trois ans par la Conférence des Chefs d'États et de gouvernements. Ce groupe de sages est chargé de fournir des services consultatifs au Conseil de paix et de sécurité et au Président de la Commission sur toutes questions relatives au maintien et à la promotion de la paix, de la sécurité et de la stabilité en Afrique. Il faut aussi mentionner le système continental d'alerte rapide composé d'un centre d'observation et de contrôle chargé de la collecte et de l'analyse des données transmises par des unités d'information et de contrôle des mécanismes régionaux, permettant de mieux suivre les foyers de tensions potentiels et lévolution des conflits.

692 DUJARDIN (Stéphanie), "L'UA : objectifs et moyens de gestion des crises politiques et des conflits armés ", op.cit., p.73. 
le génocide, les crimes contre l'humanité. ${ }^{693}$ Il est prévu par le Protocole créant le Conseil de paix et de sécurité une force africaine prépositionnée qui sera composée de contingents multidisciplinaires en attente, avec des composantes civiles et militaires, stationnées dans leurs pays dorigine et prêts à être déployés rapidement. Cette force sera dirigée par un Comité d'État major chargé de conseiller et d'assister le Conseil de paix et de sécurité pour tout ce qui concerne les questions d’ordre militaire. ${ }^{64}$ Le Conseil de paix et de sécurité est également mandaté pour entreprendre des activités de consolidation de la paix dans les situations post-conflits. ${ }^{695}$ Il facilite la restauration de l'État de droit, la création et le développement d'institutions démocratiques ainsi que la supervision des élections de l'État concerné. ${ }^{696}$ Il jouit de ce fait d'un domaine d’actions politiques très large.

$\mathrm{Au}$ regard de ses attributions politiques, le Conseil a pu s'investir dans la recherche de solutions à certaines crises politiques survenues sur le continent. Le premier test pour l'UA et le Conseil de paix et de sécurité a été le Togo. ${ }^{697}$ Afin de condamner laccession controversée au pouvoir du Président Faure GNASSINGBE en février 2005, le Conseil de paix et de sécurité a suspendu les autorités de fait du Togo de toutes les activités des organes de l'UA jusqu'au retour de lordre constitutionnel. ${ }^{698}$ Dans sa résolution du 25 février 2005, le Conseil de paix et de sécurité exige le retour à la légalité constitutionnelle qui passe par le départ de Faure GNASSINGBE et le respect des dispositions de la Constitution togolaise relatives à la succession à la tête de l'État. ${ }^{699}$ Des élections présidentielles sont organisées très rapidement en avril et Faure GNASSINGBE sort vainqueur. ${ }^{700}$ Alors, le Conseil de paix et de sécurité adopte une résolution le 27 mai 2005 dans laquelle il considère que les conditions de la levée des sanctions contre le Togo ont été remplies et retire la mesure de suspension des autorités togolaises aux activités de l'UA. ${ }^{701}$

Egalement, la gestion de la crise ivoirienne a été l'une des préoccupations du Conseil de paix et de sécurité. En fait, suite à l'attaque d'un groupe rebelle le 19 septembre 2002, la détérioration de la situation politique en Côte d'Ivoire fait peser de graves dangers sur la paix et la stabilité dans la sous région de l'Afrique de l'ouest. ${ }^{702}$ Cette situation conduit le Conseil de paix et de sécurité à se saisir du dossier en collaboration avec l'ONU et la CEDEAO. Dans sa résolution d'octobre 2005, le

693 Voir l'article 4, paragraphe h de l'Acte constitutif de l'UA.

694 Voir l'article 13 du Protocole créant le Conseil de paix et de sécurité.

695 DUJARDIN (Stéphanie), "L’UA : objectifs et moyens de règlement des crises politiques et des conflits armés », op.cit., p.74.

696 Ibid.

697 ADJOVI (Roland), « Togo, un changement anticonstitutionnel savant et un nouveau test pour l'Union africaine ", Actualité et droit international, www.ridi.org/adi, février 2005, p.1.

698 DUJARDIN (Stéphanie), "L'UA : objectifs et moyens de règlement des crises politiques et conflits armés ", op.cit.,p.76.

699 Ibid.

$700 \quad$ Ibid.

701 DUJARDIN (Stéphanie), «L’UA : objectifs et moyens de règlement des crises politiques et des conflits armés », op.cit., p.76.

Ibid, p.78. 
Conseil de paix et de sécurité réitère sa demande aux parties de s'abstenir du recours à la force ainsi que le démantèlement et le désarmement des milices. ${ }^{703}$

Dans la mesure où le mandat de l'ex-Président de la République de Côte d'Ivoire, Monsieur Laurent Gbagbo prenait fin le 30 octobre 2005, le Conseil avait demandé la création des conditions nécessaires à la tenue des élections libres, régulières et transparentes. ${ }^{704}$ Or en réalité, toutes les parties ivoiriennes ont reconnu l'impossibilité d’organiser des élections à la date prévue. ${ }^{705}$ Face à cette situation embarrassante, le Conseil de paix et de sécurité décida le 6 octobre 2005 que Monsieur GBAGBO demeurera le Chef de l'État pour une période de 12 mois.

Il convient de relever l'implication du Conseil de paix et de sécurité dans la crise du Darfour. Le Darfour est une partie de l'ouest du Soudan où le gouvernement affronte depuis février 2003, plusieurs mouvements rebelles, à l'aide des milices Janjaweed qu'il ne contrôle plus. Ces affrontements ont provoqué la mort de milliers de personnes et l'exode massif de réfugiés au Tchad. Le Conseil de Paix et de sécurité décide de renouveler le mandat de l'UA au Soudan et de porter à 6171 éléments militaires et 1560 personnels civils comprenant les bataillons de sept pays africains. ${ }^{706}$ Le but de la mission est d'assurer la mise en œuvre effective de l'accord de cessez-le-feu avec un accent particulier sur le désarmement et la neutralisation des milices Janjaweed, la protection de la population civile et la facilitation de l'acheminement de l'assistance humanitaire. ${ }^{707}$

Le Darfour n'est pas le seul cas où le Conseil de paix et de sécurité de l'UA a constitué une force de paix destinée à intervenir militairement dans un État membre. Il y a eu aussi l'intervention de cette organisation dans les îles Comores.

Invoquant des irrégularités et des actes d'intimidation lors de la campagne électorale, le gouvernement de l'Union des îles des Comores et l'UA avaient décidé de reporter les élections à Anjouan. ${ }^{708}$ Passant outre cette décision, Mohamed BACAR avait fait imprimer ses propres bulletins de vote et avait organisé des élections qu'il avait par ailleurs largement remportées. Ni Monsieur BACAR, ni le gouvernement de l'Union des Comores, nétait prêt à faire des concessions. Tous les efforts de l'UA pour dénouer la crise ont échoué, y compris les sanctions prises contre les dirigeants d'Anjouan ciblant leur liberté de circulation et leurs avoirs financiers. Après plusieurs négociations infructueuses, le Conseil de paix et de sécurité de l'UA a revu sa position sur la crise politique en proposant au gouvernement de l'Union de recourir à la force militaire. Soutenues par une coalition de troupes Tanzaniennes, soudanaises et sénégalaises mise sur pied par le Conseil de paix et de sécurité, et grâce à l'appui logistique de la Lybie, les forces gouvernementales comoriennes ont débarqué sur l'île d'Anjouan le 25 mars 2007 pour mettre fin à la rébellion de Mohamed BACAR.

DUJARDIN (Stéphanie), " L'UA : objectifs et moyens de règlements des crises politiques et des conflits armés »,op.cit.,p.78 et 79.

Ibid.

705 Ibid.

706 Ibid.

707 Ibid, p.81.

708 Les Comores se composent de trois îles principales : l'île d’Anjouan, la Grande Comore et Mohéli. 
Comme on vient de le montrer, les organes restreints prennent en charge les questions liées aux aspects sécuritaires du maintien de la paix. Mais, à cette dimension sécuritaire du maintien de la paix, il faut ajouter les aspects structurels qui néchappent pas aussi à la régulation des organes restreints.

Contrairement à la dimension sécuritaire du maintien de la paix qui ne concerne que l'absence de recours à la force armée dans les relations internationales, les aspects structurels supposent létablissement des conditions propices au développement politique, économique et social des États, le respect des droits fondamentaux des personnes. Aux Nations Unies, un organe restreint a reçu compétence des membres pour gérer les questions relevant des aspects structurels du maintien de la paix dans les relations internationales. Il s'agit du Conseil économique et social. Ces compétences résultent des dispositions de l’article 62, paragraphe 1 et 2 de la Charte.

Larticle 62, paragraphe 1 est consacré aux pouvoirs essentiels du Conseil économique et social : provoquer des études et adresser des recommandations sur les questions économiques et sociales. ${ }^{709}$ Les questions économiques et sociales internationales traitées par le Conseil relèvent de trois fonctions essentielles. ${ }^{710}$ Le Conseil sert de «tribune centrale» pour la formulation des recommandations de politique générale destinées aux États membres et à l'ensemble du système des Nations Unies, ainsi que pour le contrôle de leur application. ${ }^{711}$ Le Conseil assure la coordination générale des activités des organismes des Nations Unies et assure l’application des priorités définies par l'Assemblée générale pour l'ensemble du système. ${ }^{712}$ Le Conseil assure la programmation des activités de l'ONU dans les domaines économique et social. Dans ce dernier cas, le domaine couvert par le Conseil touche toutes les activités économiques et sociales de l'ONU.713 Il se trouve placé au cœur de la régulation des questions liées au développement économique et social des États.

Aux termes du paragraphe 2 de l'article 62 de la Charte, son domaine de compétence prend aussi en compte les questions des droits de l'homme et des libertés fondamentales. ${ }^{714}$ Elle

709 RUCZ (Claude), "Commentaire de l'article 62, paragraphe 1 de la charte des Nations Unies », in COT (JeanPierre), PELLET (Alain), La Charte des Nations Unies, commentaire article par article », op. cit., p.951.

$710 \quad$ Ibid, p.952.

711 Ibid.

712 RUCZ (Claude), « Commentaire de l'article 62, paragraphe 1 de la Charte des Nations Unies », op.cit., p.952 .

713 Ces activités économiques et sociales qui relèvent de la compétence du Conseil économique et social sont :

- Secours en cas de catastrophes, - Ressources naturelles,

- Contrôle en cas de drogues, - Cartographie,

- Réfugiés,

- Energie,

- Environnement,

- Alimentation et agriculture,

- Habitat,

- Administration publique,

- Science et technologie,

- développement social et femmes,

- Statistiques,

- Sociétés transnationales,

- Développement industriel,

- Transports.

714 Larticle 62, paragraphe 2 est ainsi libellé : « Il peut faire des recommandations en vue d'assurer le respect effectif des droits de l'homme et des libertés fondamentales pour tous.» 
était dans l'esprit des rédacteurs de la Charte un terrain d'intervention privilégiée et prioritaire. ${ }^{715}$ L'intérêt porté au respect effectif des droits de l'homme était ainsi institutionnalisé en reconnaissant au Conseil économique et social le pouvoir d'adresser des recommandations aux Etas membres et non membres. ${ }^{716}$

Le Conseil a adopté des recommandations sur l'état du respect des droits de l'homme et des libertés au Chili, après avoir mis en place un groupe de travail spécial d’enquête, de même que des rapports ont été établis sur le Kampuchea démocratique, l'Afghanistan et la Pologne, la Bolivie, le Guatemala, le Salvador et l'Iran. ${ }^{717}$

Le Conseil a aussi porté son attention sur les domaines les plus variés : condition de la femme, droits de l'enfant, libertés fondamentales garanties aux individus en matière judiciaire, respect des droits de l'homme et la dignité des travailleurs migrants, non discrimination dans le droit pour tous daller et de venir dans tout pays, questions des exécutions sommaires ou arbitraires, de la torture ou des traitements inhumains ou dégradants, discrimination à l'encontre des populations autochtones, protection des droits syndicaux, lutte contre l'esclavage et la traite des êtres humains, etc... ${ }^{718}$

Les organes restreints exercent des pouvoirs opérationnels dans les relations internationales. Cependant, l'exercice de ces pouvoirs recèle de nombreuses carences qu'il convient de mettre en lumière.

\section{B : LES CARENCES DES POUVOIRS OPERATIONNELS DES ORGANES RESTREINTS}

Parler des carences des pouvoirs opérationnels des organes restreints revient à montrer les insuffisances qui apparaissent dans la mise en ouvre de ceux-ci dans les relations internationales. Les limites qui affectent ces pouvoirs opérationnels concernent la politique du «deux poids deux mesures» pratiquée au sein de ces organes. La politique du «deux poids, deux mesures » consiste à traiter de manière différente des situations identiques sur le plan du droit international. ${ }^{719}$ C'est une politique d'engagement sélectif. ${ }^{720}$ En d'autres termes, il s'agit d'une application sélective du droit international aux États proportionnellement au capital de sympathie dont ils jouissent dans les relations internationales.

715 ROSENBERG (Dominique), "Commentaire de l'article 62, paragraphe 2 de la Charte de l'ONU », in COT (Jean-Pierr), PELLET (Alain), La Charte des Nations Unies, commentaire article par article, op.cit., p.968.

716 Ibid.

717 ROSENBERG (Dominique), "Commentaire de l'article 62, paragraphe 1 de la Charte des Nations Unies ", op.cit., p.967.

718 Ibid.

719 DJIENA WEMBOU (Michel-Cyr), Le droit international dans un monde en mutation, op.cit.,p.136.

720 FLEURENCE (olivier), La réforme du Conseil de sécurité, l'état du débat depuis la fin de la guerre froide, op.cit., p.132. 
Cette politique «du deux poids, deux mesures» remet en cause l'efficacité du système de sécurité collective des Nations Unies construit autour du Conseil de sécurité. La sécurité collective est l'engagement pris par chaque État d’apporter son appui à une décision collective de sopposer à tout État coupable, d'une agression ou d'une menace à la paix. ${ }^{721}$ Comme l'a fait observer le Professeur Michel VIRALLY, «c'est l'engagement de tous contre et de tous avec tous.» ${ }^{722}$

Mais à l'épreuve des faits, la réaction du Conseil de sécurité face à certaines menaces contre la paix et la sécurité internationales a été marquée par le triomphe des comportements sélectifs des États les plus puissants que sont les membres permanents. ${ }^{723}$ Face aux violations massives des droits de l'homme, cette réaction dépend des intérêts stratégiques des États, notamment des plus puissants d'entre eux. ${ }^{724}$ Comme l'observe le Professeur DJIENA WEMBOU, «en 1965, environ 300000 personnes dorigines chinoises furent assassinées en Indonésie. Les faits furent rapportés dans la presse. Mais aucune action ne fut organisée en leur faveur. De même, les Khmers rouges massacrèrent au vu et au su de tout le monde des millions de leurs compatriotes. Le Conseil de sécurité ne prit aucune mesure pour faire cesser les massacres.» ${ }^{725}$

La situation en Somalie constitue une autre illustration de la politique du «deux poids deux mesures» dans laquelle s'inscrit le Conseil de sécurité. En effet, «depuis le 17 novembre 1991 une guerre civile de factions ravage la ville de Mogadiscio. La quatrième en dix mois; le bilan, dressé par des Organisations Non gouvernementales aussi sérieuses qu'Human Right Watch et Médecins du Monde, sélève à 14000 morts et 27000 blessés. Mais la Somalie est un pays pauvre, sans pétrole, qui ne représente plus un intérêt stratégique pour les grandes puissances. Après bien de tergiversations, le Conseil de sécurité n’a décidé d’aucune véritable intervention humanitaire en faveur des civils somaliens.» ${ }^{726}$

Mais cette politique de «deux poids, deux mesures», n’est pas pratiquée seulement au Conseil de sécurité des Nations Unies. Elle prévaut aussi au Conseil de paix et de sécurité de l'UA. Cet organe est intervenu militairement dans l'île d'Anjouan afin de rétablir l'ordre constitutionnel qui était menacé par les actes de rébellion du Colonel Mohamed BACAR. Si cette intervention mérite dêtre relevée comme une application de l'article 4, paragraphe (p) de l'Acte constitutif de l'UA relatif au refus des changements anti-constitutionnels de gouvernement, elle suscite néanmoins des réserves assez importantes. Si elle a été effectuée, sans nul doute c'est parce qu’Anjouan est une petite île qui ne représente en réalité aucun danger du point de vue militaire. Sinon, comment comprendre que dans d'autres circonstances similaires, le Conseil de paix et de sécurité n’a pas jugé utile d’envoyer des troupes armées afin de rétablir l’ordre constitutionnel ? C’est le cas de la

721 BOOH BOOH (Jean Roger), «Les opérations onusiennes de maintien de la paix en Afrique : vision d’un acteur sur le terrain », in «L'ONU vue d’Afrique », Maisonneuve et Larosse/ Afredit, Paris, 2003, p.89.

Ibid. DJIENA WEMBOU (Michel-Cyr), Le droit international dans un monde en mutation, op.cit.,p.79. Ibid, p.79 et 80 .

Ibid.

DJIENA WEMBOU (Michel-Cyr), Le droit international dans un monde en mutation, op.cit., p.79 et 80. 
République centrafricaine où le Général François BOZIZE, parvenu au pouvoir suite à un coup d'État, resta sourd aux invitations de l'Union qui plaidait pour le retour à l'ordre constitutionnel. ${ }^{727}$ Pour des raisons similaires, les appels répétés de l'UA au retour à l'ordre constitutionnel en Mauritanie nont jamais eu pour effet le renoncement à l'exercice du pouvoir par la junte. ${ }^{728}$

La prise du pouvoir par la force de François BOZIZE en République centrafricaine et celle de la junte en Mauritanie ont donné à l’opinion publique africaine l'occasion de pouvoir mesurer l'efficacité de l'UA ${ }^{729}$ et particulièrement de son Conseil de paix et de sécurité. Mais, dans ces deux États le Conseil de paix et de sécurité n'a pas pu mettre en œuvre une intervention militaire en vue de rétablir des régimes politiques destitués par des moyens contraires au droit. Ainsi, sa crédibilité est remise en cause aux yeux de l'opinion publique africaine et de la communauté internationale. ${ }^{730}$ De ce constat, une question fondamentale mérite dêtre posée : est- ce que le Conseil de paix et de sécurité de l'UA peut effectuer une intervention militaire dans certains grands États comme le Nigéria, l’Afrique du Sud, l'Algérie, l'Egypte...etc en cas de velléités de prise de pouvoir par des moyens non démocratiques?

Les organes restreints disposent de pouvoirs politiques qui leur permettent d'agir dans les relations internationales. Qu'en est-il de leurs pouvoirs juridiques?

BIYOGUE BI NTOUGOU (Jean Delors), "Les faiblesses du système africain de prévention, gestion et de résolution des conflits ", in BANGOURA (Dominique),FIDIECK A BIDIAS, L'Union Africaine et les acteurs sociaux dans la gestion des crises et des conflits armés ", L'Harmattan, Paris, 2005, p.86. Ibid.

729 Ibid, p.88.

730 Ibid. 


\section{CHAPITRE II : LES POUVOIRS JURIDIQUES DES ORGANES RESTREINTS.}

Les pouvoirs juridiques peuvent s'entendre comme des compétences reconnues par la Charte constitutive des organisations internationales aux organes restreints pour édicter des actes juridiques. Afin de saisir son contenu réel, il convient de mettre en exergue la nomenclature (Section I) et la valeur juridique des actes juridiques édictés par les organes restreints (Section II). 
Lactivité normative des organes restreints comprend les actes juridiques liés au fonctionnement interne (Paragraphe I) et ceux régissant les activités extérieures de lorganisation (Paragraphe II).

\section{PARAGRAPHE I : LES ACTES JURIDIQUES LIES AU FONCTIONNEMENT INTERNE DE L'ORGANISATION}

De prime abord, il faut souligner l'existence d'un droit interne propre aux organisations internationales. Elles ont un pouvoir d'auto-détermination, c'est-à-dire le pouvoir de déterminer elles mêmes la manière suivant laquelle elles devaient exercer leurs propres attributions. ${ }^{731}$ C'est un pouvoir règlementaire reconnu aux organes restreints.

D’une organisation internationale à une autre, la compétence règlementaire peut être attribuée par l'Acte constitutif soit aux organes pléniers, soit aux organes restreints. Le pouvoir règlementaire des organes restreints des organisations internationales sétend aux mesures individuelles (A) et aux mesures générales (B).

\section{A: LES MESURES INDIVIDUELLES}

Les mesures individuelles concernent la nomination et la révocation des agents, des juges des juridictions internationales rattachées aux organisations internationales, l'admission des États et leur exclusion de l'organisation. ${ }^{732}$ Dans l'ordre juridique interne des organisations internationales, les pouvoirs visant lédiction de mesures individuelles sont reconnus à certains organes restreints.

Le Conseil de sécurité adopte des actes relatifs au statut des États membres. Ces actes portent sur l'admission, la suspension, l'exclusion des États membres, auxquelles s'ajoutent la nomination du Secrétaire général, et lélection des membres de la CIJ. Les actes relatifs à l’admission, à la suspension et à l'exclusion des États membres sont prévus par les dispositions de l’article 4 alinéa

731 FOCSANEANU (Lazare), « Le droit interne de l'organisation des Nations Unies », AFDI, 1957, p.315.

732 YAO-N'DRE (Paul), Droit des organisations internationales, op.cit., p.84. 
$2^{733}$ et $5^{734}$ et $6^{735}$ de la Charte des Nations Unies. Relativement à la nomination du Secrétaire général et la nomination des juges de la CIJ, ce sont respectivement les dispositions de l'article 97 de la Charte ${ }^{736}$ et l'article 4 du Statut de la CIJ qui les prévoient. Il ressort de ces dispositions que le Conseil prend ces actes qui sont des recommandations concurremment avec l'Assemblée générale. ${ }^{737}$ Est-ce que de telles compétences juridiques ont été reconnues au Conseil de paix et de sécurité de l'UA ? Evidemment la réponse à cette question est négative dans la mesure où la Conférence des Chefs d'État et de gouvernement qui est l'organe suprême de l'UA n'a pas partagé ses pouvoirs avec un autre organe de l'organisation. C'est elle qui décide seule des questions relatives au statut des États membres et à la nomination des hauts fonctionnaires de l'organisation. En revanche, le Président de la Commission de l'UA dispose d'un pouvoir de nomination du personnel administratif de cet organe. Aux termes de l'article 8 alinéa 1 paragraphe alinéa r), le Président de la Commission est chargé de «nommer le personnel de la Commission, conformément aux dispositions de l’article 18 des présents Statuts... $»^{738}$

Le Conseil d'administration de l'OIT dispose aussi d'un pouvoir d'édiction de mesures individuelles important. Aux termes de l'article 8 de la Constitution de l’OIT, le Directeur général du Bureau international du travail est «désigné par le Conseil d’administration, de qui il recevra ses instructions et vis-à-vis de qui il sera responsable de la bonne marche du Bureau ainsi que de l'exécution de toutes autres tâches qui auront pu lui être confiées... » ${ }^{739}$

En dehors de l'édiction de mesures individuelles reconnues à certains organes restreints, il y a les mesures générales qui concernent le fonctionnement de l’organisation.

\section{B: LES MESURES GENERALES}

Les mesures générales reconnues aux organes restreints des organisations internationales sont celles qui organisent les conditions de travail et les relations entre agents, entre organes d'une

733 L'article 4 alinéa 2 de la Charte des Nations Unies est libellé comme suit : «... l’admission comme membre des Nations Unies de tout État remplissant ces conditions se fait par décision de l’Assemblée générale sur recommandation du Conseil de sécurité. »

734 Larticle 5 de la Charte des Nations Unies est libellé comme suit : « Un membre de lorganisation contre lequel une action préventive a été entreprise par la Conseil de sécurité peut être suspendu par l’Assemblée générale, sur recommandation du Conseil de sécurité, de l’exercice des droits et privilèges inhérents de la qualité de membre. L’exercice de ces droits et privilèges peut être établi par le Conseil de sécurité.

735 Larticle 6 de la Charte des Nations Unies est libellé comme suit : «Si un membre de l'organisation enfreint de manière persistante les principes contenues dans la présente Charte, il peut être exclu de lorganisation par l’Assemblée générale sur recommandation du Conseil de sécurité. »

736 Larticle 97 de la Charte est libellé comme suit : «...Le Secrétaire général est nommé par l’Assemblée générale sur recommandation du Conseil de sécurité..."

737 La notion de recommandation sera clarifiée dans les analyses qui vont suivre.

738 Voir l’article 8 paragraphe 1 alinéa r) du Statut de la Commission de l'UA.

739 Voir l'article 8 de la Constitution de l'OIT. 
part, et entre agents et organes d'autre part. ${ }^{740} \mathrm{~A}$ l’opposé des mesures individuelles, les mesures générales ne visent pas un membre particulier de l’organisation encore moins un agent.

Elles concernent essentiellement les règlements intérieurs des différents organes, les règlements financiers, le statut des agents, le statut des organes subsidiaires. ${ }^{741}$ Mais dans le cadre de cette thèse, nous nous limiterons aux règlements intérieurs des organes restreints.

De façon générale, les chartes constitutives des organisations internationales reconnaissent aux organes restreints la compétence juridique d'édicter leurs propres règlements intérieurs. Le règlement intérieur d'un organe est un acte juridique qui définit la procédure de travail en son sein.

Larticle 30 de la Charte de l'ONU dispose que «le Conseil de sécurité établit son Règlement intérieur, dans lequel il fixe le mode de désignation de son président.» ${ }^{742}$ Mais un fait important relatif à ce Règlement intérieur mérite dêtre souligné. En effet, quand le Conseil de sécurité se réunit pour la première fois à Londres le 17 janvier 1946, il n’avait pas encore de règlement intérieur. ${ }^{743}$ Le Règlement intérieur fut rédigé par un comité d’experts créé par le Conseil de sécurité et fut publié le 5 février $1946 .{ }^{744}$ Mais, précise l’Ambassadeur Jacques LEPRETTE, «assez curieusement, le Règlement intérieur, après plus de quarante-quatre ans demeure un Règlement «provisoire ». Pourquoi ? Il semble que les membres du Conseil, et très particulièrement ses membres permanents aient préféré ne pas conférer à ces règles de procédure une valeur intangible et ne pas courir le risque, en tentant de rendre le Règlement définitif, douvrir des controverses inutiles et probablement sans solution.» ${ }^{745}$

Relativement au Conseil économique et social, l’article 72 de la Charte précise qu’il «1. Adopte son Règlement intérieur, dans lequel il fixe le mode de désignation de son Président.

2. Il se réunit selon les besoins, conformément à son Règlement, celui-ci comportera des dispositions prévoyant la convocation du Conseil sur la demande de la majorité de ses membres.» ${ }^{746}$

Dans la même logique, l'article 14 du Protocole relatif au Conseil de paix et se sécurité dispose aussi qu'il « établit son propre Règlement intérieur, dans lequel il fixe la convocation de ses réunions, la conduite des débats, la publicité et les procès verbaux des séances, ainsi que tout autre aspect pertinent de son travail, pour examen et approbation par la Conférence.» ${ }^{747}$

Enfin, le Règlement du Conseil d'administration de l'OIT a été adopté le 23 mars 1920. Il a connu plusieurs modifications dont la dernière en date est celle du 18 novembre $1999 .{ }^{748}$

\footnotetext{
740 YAO-N’DRE (Paul), Droit des organisations internationales, op.cit.,p.84.

741 Ibid.

742 Voir l'article 30 de la Charte des Nations Unies.

743 LEPRETTE (Jacques), « Commentaire de l'article 30 de la Charte des Nations Unies. », in COT (Jean-Pierre), PELLET (Alain), La Charte des Nations Unies, commentaire article par article », $2^{\mathrm{e}}$ édition, op.cit., p.544.

$744 \quad$ Ibid.

745 Ibid, p.543-544

746 Voir l'article 72 de la Charte des Nations Unies.

747 Voir l'article 14 du Protocole relatif à création du Conseil de paix et de sécurité de l'UA.

748 Voir le Règlement du Conseil d’administration de l'OIT, Genève, février 2000.
} 
$\mathrm{Au}$ plan juridique les organes restreints peuvent prendre des mesures individuelles et des mesures générales qui ne sont que des actes juridiques relatifs au fonctionnement interne des organisations internationales. Mais, à côté de ceux-ci il faut ajouter les actes juridiques liés aux activités extérieures des organisations internationales.

\section{PARAGRAPHE II : LES ACTES JURIDIQUES LIES AUX ACTIVITES EXTERIEURES DES ORGANISATIONS INTERNATIONALES}

Les compétences juridiques des organes restreints ne se limitent pas au fonctionnement interne des organisations internationales. Une grande partie de leurs actes juridiques régissent leurs activités extérieures. Ces actes se divisent en deux grandes catégories. Ce sont les actes unilatéraux (A) et les actes conventionnels (B).

\section{A : LES ACTES UNILATERAUX}

Qu'est ce qu'un acte unilatéral ? Le Professeur YAO-N'DRE Paul définit un acte unilatéral comme «une manifestation de volonté émanant d’un sujet de droit international et destiné à produire des droits et des obligations dans les relations internationales.» ${ }^{749}$ Les actes unilatéraux peuvent procéder des organes juridictionnels, des États et des organisations internationales. ${ }^{750} \mathrm{Il}$ comprennent les résolutions (1), des recommandations (2) et des décisions (3) que nous allons analyser successivement.

\section{1/ Les résolutions des organes restreints}

Plusieurs auteurs ont donné une approche définitionnelle des résolutions des organisations internationales. Les résolutions, selon le Professeur YAO-N’DRE «sont des actes qui émanent généralement des organes intergouvernementaux des organisations internationales et qui suggèrent aux destinataires une ligne de conduite à tenir». ${ }^{751}$

Quant au Professeur Jorge CASTENADA, il soutient que «le contenu de ce concept est trop vaste : une résolution peut comporter soit un ordre, soit une invitation, soit une variété de formes hybrides intermédiaires; elle traite soit des questions techniques, soit d’affaires éminemment politiques ; tantôt elle a un caractère matériellement législatif, c'est-à-dire qu'elle énonce des normes juridiques, tantôt elle constitue un acte administratif individuel [...] Quel que soit l'angle sous lequel on l'examine, le concept de résolutions internationales n'est pas univoque.» ${ }^{752}$

\footnotetext{
749 YAO-N’DRE (Paul), Relations internationales, Abidjan, PUCI, avril 1999, p.148.

750 Ibid.

751 YAO-N’DRE (Paul), Droit des organisations internationales, Abidjan, PUCI, 1996, p.107.

752 CASTENADA (Jorge), « La valeur juridique des résolutions des Nations Unies », RCADI, 1970-I, p.211.
} 
Dans les organisations internationales, les résolutions peuvent être édictées par les organes pléniers et les organes restreints. Dans le cas du Conseil de sécurité, l'initiative d'un projet ou d'une proposition de résolution échoit aux membres, permanents et non permanents. En revanche, tout membre des Nations Unies convié à participer aux débats du Conseil de sécurité au titre de l’article 32 de la Charte peut présenter des propositions et des projets de résolution. ${ }^{753}$ Lélaboration d'une résolution est souvent un long chemin. Elle est en général une solution à un problème qu'il a luimême soulevé ou qui lui a été soumis par le Secrétaire général, par l’Assemblée générale ou par un État membre de l'ONU. Elle définit les actions à entreprendre pour résoudre ce problème. Il peut donc se passer plusieurs mois avant qu'un projet de résolution ne soit rédigé, discuté, voté puis appliqué. ${ }^{754}$ Aux termes de l'article 31 du Règlement intérieur du Conseil de sécurité, «les projets, les amendements et les propositions de fond sont en principe soumis aux représentants par écrits.» ${ }^{755}$ Dans la suite des débats au Conseil, selon l’article 32 du Règlement intérieur, «les propositions principales et les projets de résolutions ont priorité dans l'ordre où ils sont présentés... " ${ }^{756}$ Mais seuls peuvent alors venir en priorité les suspensions ou ajournements de séance, le renvoi à une Commission, au Secrétaire général ou l'introduction d'un amendement. ${ }^{757}$

En ce qui concerne l'adoption d'une résolution proprement dite, il est important de souligner quelle fait l'objet de nombreuses tractations politiques et diplomatiques entre les États membres. Généralement, on assiste à un marchandage des votes entre les membres permanents et les membres non permanents. Lancien Secrétaire général des Nations Unies, BOUTROSGHALI sétale largement sur cette question. En effet, il cite les « procédés diplomatiques habituels : persuasion, pression, assistance...», ensuite «le rayonnement politique et économique de la grande puissance» et enfin «les accords et organismes régionaux [...] qui imposent à leurs membres une certaine discipline au profit des grandes puissances...» ${ }^{758}$ Le vote des résolutions au sein des organes restreints des organisations internationales est souvent le résultat de pressions politiques des grandes puissances sur les petits États.

En guise d'exemple, les discussions qui ont entouré le vote de la résolution 1721 du Conseil de sécurité des Nations Unies sur la crise ivoirienne. La France, membre permanent du Conseil de sécurité a usé de son poids politique pour influencer l'adoption de cette résolution. Cette attitude de la France a été fustigée par Monsieur John BOLTON, ancien Ambassadeur des États-Unis à l'ONU. En effet, le diplomate américain soutient : «Par bien des aspects, je pense que la France et les Européens en général se comportaient comme si la Côte d’Ivoire était encore une colonie. 
Ils administraient ses affaires, décidaient de qui devaient diriger le pays ; en fait, ils décidaient des moindres aspects de la situation politique interne en Côte d'Ivoire.» ${ }^{759}$

La France a voulu intentionnellement changer la pratique habituelle au Conseil de sécurité selon laquelle lorsque «l'UA trouve un accord, elle vient au Conseil de sécurité et lui demande simplement de l'adopter tel quel.» ${ }^{760}$ Or selon John BOLTON, «la position française marquait donc une différence flagrante avec la manière dont nous avons procédé auparavant. Cela peut ne pas être une mauvaise chose, et je ne pense que l'UA ait toujours raison. Mais soyons claire : ceci était une déviation par rapport à la pratique habituelle...» ${ }^{761}$

La France, membre permanent du Conseil de Sécurité use de toute son influence politique et diplomatique en vue d'obtenir le vote d'une résolution correspondant à ses aspirations Dans cette perspective, elle réussit à rallier à sa cause le Secrétaire général de l'ONU -Monsieur Koffi ANAN-, qui pour John BOLTON «a très clairement pris fait et cause pour la position de la France. Et John Bolton révèle que «l'un de ses adjoints est venu dire au Conseil : «Nous devrions soutenir le texte français. Je lui ai demandé : est-ce que c'est la position du Secrétaire que seul le texte français est acceptable ? Il y eut un long silence, parce qu'ils n'avaient pas l'habitude dêtre contredits sur des choses de ce genre.» ${ }^{762}$

Cet exemple témoigne de l'environnement politique qui entoure les résolutions des organes restreints. Que pouvons-nous dire sur les recommandations?

\section{2/ Les recommandations des organes restreints}

Le concept de recommandation constitue sans doute l'un des plus utilisé dans le langage $\mathrm{du}$ droit des organisations internationales. Le terme de recommandation, apparu d'abord dans la pratique des conférences diplomatiques, est devenu d'usage constant dans le droit des organisations internationales. ${ }^{763}$

Cependant, il est important de savoir ce que recouvrent en réalité les recommandations des organisations internationales. Pour le Professeur Michel VIRALLY «le terme de recommandation peut dès lors qualifier les résolutions d'un organe international adressées à un ou plusieurs destinataires qui lui sont extérieurs et impliquant une invitation à adopter un comportement déterminé, action ou abstention.» ${ }^{764}$

Quant au Professeur Jorge CASTENADA, il établit un lien entre les recommandations des organisations internationales et la souveraineté des États dans l'ordre international. Pour lui,

\footnotetext{
759 Voir le quotidien ivoirien «Le Courrier d'Abidjan » dans sa parution du mardi 24 juillet 2007, p.6 et 10.

760 Ibid.

761 Ibid.

762 Ibid.

763 VIRALLY (Michel), « La valeur juridique des recommandations des organisations internationales », AFDI, 1956, p.66.

$764 \quad$ Ibid, p.68.
} 
«lordre international en raison de sa structure embryonnaire fondée sur la souveraineté des États, et vu la nécessité de recourir, dans chaque cas, à la collaboration des ses membres pour l'exécution des obligations internationales, il est naturel que la forme normale d'expression et l'instrument type de la société internationale soient la recommandation...» ${ }^{765} \mathrm{Il}$ en découle que dans les rapports entre États et organisations internationales, l’instrument privilégié de la collaboration est la recommandation.

Les fins désignées par un traité institutionnel ne peuvent quêtre générales et abstraites. Il reste à leur donner un contenu concret de règles d'action et de solutions précises en face des multiples problèmes que font naître la vie et les relations des États. ${ }^{766}$ C'est à travers les recommandations des différents organes que l'organisation exprime sa volonté politique dans les relations internationales.

Dans la Charte des Nations Unies le pouvoir de recommandation est reconnu au Conseil de sécurité dans certains domaines importants. Comme l'a souligné le professeur Michel VIRALLY, la Charte « réserve certains actes importants pour l'avenir de l'organisation à une décision exigeant l'accord des deux grands organes politiques : l'admission d'un nouveau membre, la suspension ou l'exclusion d'un membre, la nomination du secrétaire général résultent d'une décision de l'Assemblée générale prise sur recommandation du Conseil de Sécurité ». ${ }^{767}$

Toutes les décisions importantes de l'Assemblée générale sont conditionnées à une recommandation du Conseil de sécurité. Or toute recommandation du Conseil de sécurité néchappe pas au droit de veto des membres permanents. D’où la très grande politisation de ces

765 CASTENADA (Jorge), « La valeur juridique des résolutions des Nations Unies », RCADI, 1970-I, Tome 129, p.218.

766 VIRALLY (Michel), « La valeur juridique des recommandations des organisations internationales », op.cit., p.83.

$767 \quad$ Ibid, p.76. 
questions essentielles telles que la nomination d'un nouveau Secrétaire général, l’admission et lexclusion de certains États membres... ${ }^{768}$

\section{3/ Les décisions des organes restreints}

Le Professeur NGUYEN QUOC Dinh définit la décision comme «un acte unilatéral qui émane d'une manifestation de volonté de l'organisation et qui produit des effets juridiques à l'égard de son ou de ses destinataires.» ${ }^{769}$ Elle émane d'un organe d'une organisation internationale et a nécessairement un effet obligatoire.

C'est précisément dans le cadre du maintien de la paix et de la sécurité internationale et aussi dans le cadre des mesures d'exécution des arrêts de la $\mathrm{CI}{ }^{770}$ que le Conseil de sécurité prend des décisions. ${ }^{771}$ Mais, en ce qui concerne les décisions prises dans le domaine du maintien de la paix une analyse s'impose. Le maintien de la paix constitue une prérogative de puissance publique reconnue au Conseil de sécurité. ${ }^{72}$ Par le truchement du maintien de la paix, le Conseil de sécurité devient ainsi le gendarme international qui a charge de concevoir et d'imposer l'ordre mondial. ${ }^{773}$

768 Ce point a soulevé de vives controverses au sein de l’Assemblée générale à la suite du blocage de la procédure d'admission provoqué par l'usage systématique du droit de veto au Conseil de sécurité. Le Conseil sétant divisé en deux blocs se trouve désormais incapable de statuer objectivement et individuellement sur les candidatures. Les États soutiennent les candidats de leur bloc et barrent ceux de l'autre : l'absence de majorité, le veto paralysant les admissions. De plus, certains pays proposent l'admission globale de tous les candidats (package deal), ou subordonnent leur vote pour un État à la condition que d'autres États soient admis. L'Assemblée générale sétant émue de ces difficultés adopte une résolution du 17 novembre 1947 demandant un avis à la Cour sur deux questions :

«Un membre de l'ONU appelé en vertu de l’article 4 à se prononcer par son vote, soit au Conseil de sécurité, soit à l'Assemblée générale, sur l’admission d’un État comme membre des Nations Unies, est-il juridiquement fondé à faire dépendre son consentement à cette admission de condition non expressément prévues à l'alinéa $1^{\text {er }}$ de l'article 4 ?»

«En particulier peut-il, alors qu’il reconnaît que les conditions prévues par ce texte sont remplies subordonner son vote affirmatif à la condition que, en même temps que l'État dont il s'agit, d’autres États soient également admis comme membres des Nations Unies?"

A la première question, la Cour répond que l'article 4 de la Charte pose des conditions nécessaires, mais suffisantes, limitatives, et exclut les conditions politiques qui ne s'y rattacheraient pas raisonnablement.

Sur la deuxième question, il montre que l'article 4, non seulement interdit toute condition supplémentaire, mais exige l'examen individuel de ces conditions pour chaque pays, et exclut donc la condition que d'autres États soient admis en même temps.

769 NGUYEN QUOC (Dinh), Droit international public, op.cit.,p.365 et 366.

$770 \quad$ Il s'agit ici de l’article 94 , paragraphe 2.

771 Il faut souligner que dans le cadre du Chapitre VII, le Conseil de Sécurité peut librement choisir le procédé de la recommandation où s'engager dans la voie des décisions. Toutefois, lorsque le Conseil de Sécurité ordonne un cessez-le-feu ou exige une cessation immédiate des hostilités et le retrait des forces militaires d'un territoire étranger, c'est la voie de la décision qu'il utilise. C’est le cas également lorsqu'il décide de déclencher des sanctions au titre de l'article 41 à l'encontre d'un État membre ou non membre.

772 DEGNI-SEGUI (René), «Commentaire de larticle 24 paragraphe 1 et 2 de la Charte des Nations Unies », op. cit., p.450.

Ibid, p.447 
Cependant, au delà du Conseil de sécurité, c'est, en définitive aux grandes puissances qu’est dévolu le rôle principal du maintien de la paix. Celle-ci ne peut être maintenue sans leur appui financier et militaire. ${ }^{74}$ Mieux, le droit de veto dont l'exercice relève d'un pouvoir discrétionnaire, vient davantage renforcer leur poids politique et juridique, si bien quaucune décision ne peut être prise par le Conseil sans le consentement de l'un quelconque du "club des cinq" et à fortiori contre eux. ${ }^{775}$ Le pouvoir de décision du Conseil de Sécurité est un instrument de domination des grandes puissances dans les relations internationales. Egalement, l'Acte constitutif de l'UA reconnait au Conseil de paix et de sécurité le pouvoir d’édicter des décisions. Cette compétence apparaît à l'article 7, paragraphe 3 du Protocole relatif à la création du Conseil de paix et de sécurité en ces termes : « les États membres conviennent d'accepter et d’appliquer les décisions du Conseil de paix et de sécurité, conformément à l’Acte constitutif. ${ }^{776}$

Les organes restreints des organisations internationales disposent de la compétence dédicter des actes unilatéraux. Mais en dehors de ceux-ci, il faut aussi mentionner que ces organes ont aussi le pouvoir de conclure des traités.

\section{B : LA CONCLUSION DES TRAITES}

Le Professeur N'Guyen QUOC DINH définit le traité comme «un accord conclu entre deux ou plusieurs sujets de droit, régit par le droit international et destiné à produire des effets de droit.» La définition donnée par le Professeur SYKES s'inscrit dans la même logique. Selon lui, «a treaty is an agreement executed by duly authorised officials of signatory states, evincing an intention to make it binding legal obligation. Treaty obligations are themselves governed by an over-aching treaty known as the Vienna Convention on treaty...» ${ }^{777}$

Les organisations internationales, en tant que sujet de droit international public, ont le pouvoir de conclure des traités avec les autres sujets de droit international que sont les États et aussi les organisations internationales. ${ }^{778}$

Cependant, la capacité reconnue aux organisations internationales de conclure des traités soulève une question fondamentale : quels sont, dans les organisations internationales, les

$774 \quad$ Ibid, p.450 et 451.

775 Ibid, p.451.

776 Voir l'article 7 paragraphe 3 du Protocole relatif à la création du Conseil de paix et de sécurité de l'UA.

777 SYKES (Alan O.), «The economics of public international law », John M. Olin law and economics working paper, $\mathrm{n}^{\circ} 216,2^{\text {nd }}$ series, Chicago, July, 2004, p.3.

778 Pour les règles applicables aux traités conclus par les organisations internationales, il faut se référer aux dispositions de la Convention de Vienne sur le droit des traités entre États du 23 mai 1969 et aux dispositions de la Convention de Vienne de 1986 sur le droit des traités conclus entre États et organisations internationales ou entre deux ou plusieurs organisations internationales du 21 mars 1986. 
organes compétents pour les engager définitivement ${ }^{779}$ Les organes restreints des organisations internationales ont-ils le pouvoir juridique de conclure un traité?

La réponse que nous donnerons à ces différentes questions nous conduira à suivre la démarche du Professeur René-Jean DUPUY dans son cours donné à l'académie de droit international de la Haye en $1960 .{ }^{780}$ Pour le Professeur René-Jean DUPUY, il faut distinguer formellement au sein des organisations internationales « les organes compétents pour élaborer les traités et ceux qui sont compétents pour son entrée en vigueur. »

Lélaboration des traités, de façon générale concerne la négociation, la rédaction et la signature. ${ }^{781}$ La Convention de Vienne de 1986, en son article 7 précise que l’autorité compétente pour négocier, signer et exprimer l'accord de l'organisation à être lié ne bénéficie pas d'une présomption. Elle doit produire des pouvoirs appropriés pour établir quelle possède de telles compétences pour engager l’organisation. ${ }^{782}$ Larticle 6 de cette Convention précise que la capacité de conclure des traités d'une organisation est régie par les dispositions pertinentes de son existence. ${ }^{783}$ Aux termes de ces deux dispositions, il faut admettre qu'aucun organe de l'organisation ne bénéficie d’office de la capacité d'élaborer un traité. Le pouvoir d'élaboration d'un traité au sein d'une organisation internationale peut être valablement reconnu aussi bien à un organe plénier quà un organe restreint.

Dans le cadre du système des Nations Unies, la Charte de l'organisation dispose en son article 63 que «le Conseil économique et social peut conclure avec toute institution visée à l'article 57 des accords fixant les conditions dans lesquelles cette institution sera reliée à l'organisation»; mais cette disposition précise que «ces accords sont soumis à l'approbation de l'Assemblée générale». ${ }^{784}$ De cette disposition de la Charte des Nations Unies, il est nécessaire de mettre en évidence le pouvoir attribué par la Charte spécifiquement à cet organe restreint de conclure les accords de liaison et de coordonner l'activité des institutions spécialisées. ${ }^{75}$ De ce fait, le Conseil économique et social devient le centre moteur du système de coordination externe avec les autres organisations internationales. ${ }^{786} \mathrm{Il}$ a reçu compétence pour négocier, rédiger et signer les accords de liaison avec les institutions spécialisées. Il a institué au cours de sa première session un comité chargé des négociations avec les institutions intergouvernementales composé de onze membres. Ce comité a mené, à quelques exceptions près, les négociations avec les institutions spécialisées. Il a

\footnotetext{
779 DUPUY (René-Jean), « Le droit des relations entre les organisations internationales », RCADI, 1960-II, p.492.

$780 \quad$ Ibid, p. 489 à 514.

781 Ibid,p.493.

782 YAO-N'DRE (Paul), Droit des organisations internationales, op.cit.p.108.

783 Ibid.

784 Voir l'article 63 de la Charte de l'ONU.

785 MANIN (Aleth), "Commentaire de l'article 63 de la Charte des Nations Unies ", in COT (Jean-Pierre), PELLET (Alain), La Charte des Nations Unies, commentaire article par article, $2^{\mathrm{e}}$ édition, op,cit.,p. 977.

786 MONACO (Riccardo), « Les principes régissant les structure et le fonctionnement des organisations internationales », op. cit.p. 172 .
} 
bénéficié du concours du Secrétariat général des Nations Unies qui a préparé la plupart des projets d’accords en consultation avec les Secrétariats des institutions spécialisés. ${ }^{787}$

Ainsi qu’on le constate, la négociation des accords de liaison relève de la compétence du Conseil économique et social. Comprenant un nombre retreint de membres, il est supposé agir au nom de l'ensemble des États membres. C'est à ce titre que le Conseil économique et social reçoit compétence de la Charte pour élaborer les accords entre les Nations Unies et les institutions spécialisées. ${ }^{788}$

Toutefois, la question se pose de savoir si le Conseil économique et social, après avoir élaboré les traités portant sur les accords de liaison avec les institutions spécialisées est aussi compétent pour engager définitivement l'organisation ? Cette question met en cause la distribution du pouvoir au sein de l'organisation. ${ }^{789}$ En effet, il s’agit de préciser l’organe compétent pour engager définitivement l'organisation? Un tel organe est celui qui exerce effectivement la «treaty making power» ${ }^{790}$ ou encore le pouvoir de conclure les traités.

Le comité des négociations, après avoir authentifié -signé ou paraphé- le projet d’accord avec le Chef de délégation de l'institution spécialisée, la transmet au Conseil économique et social pour adoption. ${ }^{791}$ Par la suite, le Conseil économique et social procède à l'examen du projet d'accord avant de le recommander pour approbation à l’Assemblée générale. ${ }^{792}$ L'Assemblée générale qui est lorgane plénier des Nations Unies est la seule autorité compétente pour engager définitivement l’organisation..$^{793}$ L’entrée en vigueur des accords de liaison est subordonnée à son approbation et à celle de l'organe plénier de l'institution spécialisée. ${ }^{794}$ Par conséquent, toute autre autorité agissant pour le compte de l'Assemblée générale, l’organe suprême des Nations Unies, n'est qu’un «pouvoir commis», placé sous son étroite dépendance, si bien qu’en réalité ceest cet organe qui conclut luimême l'accord. ${ }^{795}$

MANIN (Aleth), « Commentaire de l'article 63 de la Charte des Nations Unies », op.cit., p.981.

788 DUPUY (René Jean), « Le droit des relations entre les organisations internationales », op.cit., p.494 et 498.

789 Ibid, p.499.

790 Ibid.

791 MANIN (Aleth), «Commentaire de l'article 63 de la Charte des Nations Unies », op.cit., p.981.

792 Ibid.

793 DUPUY (René-Jean), « Le droit des relations entre les organisations internationales », op.cit., p.499.

794 MANIN (Aleth), «Commentaire de l'article 63 de la Charte des Nations Unies », op.cit., p...982.

795 DUPUY (René-Jean), « Le droit des relations entre les organisations internationales », op.cit., p.502. 
De ce qui précède, il faut déduire que le Conseil économique et social jouit en matière délaboration d’accord de liaison de compétences déléguées. ${ }^{796}$ C’est à l’Assemblée générale qu'appartient le dernier mot ; c'est son approbation qui engage l'organisation.$^{797}$ Lobjectif poursuivi au plan politique est une volonté de démocratisation dans la conclusion des traités ou des accords de liaison, en associant largement tous les États membres de l'organisation, représentés au sein de l’organe plénier. Le Professeur René-Jean DUPUY souligne à ce sujet que «dans les organisations interétatiques, on voudrait ainsi sauver l'influence des États ; les Assemblées plénières réalisent la démocratie directe à leur profit. Cependant les besoins de la pratique imposeront à lorgane général de désigner une assemblée réduite à un petit nombre de personnes pour conduire les négociations et préparer le texte de l'instrument. Pour autant il ne s'agira que d’un pouvoir commis, placé sous la dépendance de l’Assemblée plénière.» ${ }^{798}$

Cependant, s'il vrai que les accords conclus par le Conseil économique et social avec les institutions spécialisées sont soumis à l’approbation de l'Assemblée générale, il en va différemment de ceux conclus par le Conseil de sécurité. Aux termes de l’article 43 de la Charte des Nations Unies, «1. Tous les membres des Nations Unies, afin de contribuer au maintien de la paix et de la sécurité internationales, s'engagent à mettre à la disposition du Conseil de sécurité, sur son invitation et conformément à un accord spécial ou des accords spéciaux, les forces armées, l'assistance et les facilités, y compris le droit de passage, nécessaires au maintien de la paix et de la sécurité internationales $[\ldots .$.

3. Laccord ou les accords seront négociés aussitôt que possible, sur l'initiative du Conseil de sécurité. Ils seront conclus entre le Conseil de sécurité et des membres de l'organisation, ou entre le Conseil de sécurité et des groupes de membres de lorganisation, et devront être ratifiés par les États signataires selon leurs règles constitutionnelles respectives.» ${ }^{799}$

Cette disposition de la Charte met en exergue la compétence reconnue au Conseil de sécurité pour conclure des accords avec les États membres. Ces accords sont négociés et conclus par le Conseil de sécurité avec les États membres, des groupes d’États membres. Mais, ce texte ne précise pas si le Conseil de sécurité est compétent pour engager définitivement l’organisation ou

796 Cette primauté de l'Assemblée générale sur le Conseil économique et social en matière de conclusion d’accords de liaison trouve son fondement, en dehors de l'article 63, dans certaines dispositions de la charte des Nations Unies, notamment les articles 10 et 17. En effet, aux termes de l'article 10 de la charte : "l'Assemblée générale peut discuter toutes questions ou affaires rentrant dans le cadre de la présente charte ou se rapportant aux pouvoirs et fonctions de l’un quelconque des organes prévus dans la présente charte... ».De cette disposition, il faut retenir que l’Assemblée générale dispose d’une compétence générale lui permettant de faire des recommandations sur les autres organes principaux. Quant à l'article 17, précisément en son alinéa 3 il dispose clairement que : "l’Assemblée générale examine et approuve tous arrangements financiers et budgétaires passés avec les institutions spécialisées visées à l'article 57 et examine les budgets administratifs desdites institutions en vue de leur adresser des recommandations. »

797 DUPUY (René-Jean), « Le droit des relations entre organisations internationales », op.cit., p.51O.

$798 \quad$ Ibid, p.494.

799 Voir l'article 43 de la Charte des Nations Unies. 
s’il doit se référer à l'Assemblée générale ? Dans le silence de la Charte, on peut déduire que le Conseil de sécurité est compétent pour engager définitivement lorganisation. Dans le système de la Charte, le Conseil de sécurité est l'organe compétent en matière de manitien de la paix et de la sécurité internationale. Il serait illogique que les accords qu'il conclut avec les États membres soient soumis à l’approbation de l’Assemblée générale. Mais quelle est la valeur juridique des actes édictés par les organes restreints?

\section{SECTION II : LA VALEUR JURIDIQUE DES ACTES DES ORGANES RESTREINTS}

La valeur juridique des actes des organes des organisations internationales en générale est une question récurrente en droit international public. Il s'agit de s'interroger sur les effets produits par ces actes.Une meilleure approche de la question commande que nous envisageons la valeur juridique des actes des organes restreints dans le fonctionnement interne des organisations internationales (Paragraphe I) et dans les relations avec les États (Paragraphe II).

\section{PARAGRAPHE I : LA VALEUR DES ACTES DES ORGANES RESTREINTS DANS LE FONCTIONNEMENT INTERNE DES ORGANISATIONS INTERNATIONALES}

Les actes des organes qui sont liés au fonctionnement interne des organisations constituent les actes juridiques d’ordre intérieur. ${ }^{800}$ Ici, auteur et destinataire sont placés dans le cadre d'une seule structure institutionnelle.

Leur valeur juridique dépend de la nature des relations structurelles existant entre les organes intéressés. ${ }^{801}$ De ce point de vue, il s'agira d'analyser la valeur des actes édictés entre organes hiérarchisés (A) et celle des actes émanent des organes indépendants (B). ${ }^{802}$

800 Voir létude menée par le Professeur Lazare FOSCANEANU dans l’annuaire français de droit international de 1957 sur la question du droit interne des Nations Unies, précisément de la page 315 à la page 349. Lauteur souligne la lente prise de conscience de la doctrine sur l'existence d'un droit interne des organisations internationales. Cet état de fait s’explique selon lui par deux raisons. Il s’agit d'une part du rôle presque' exclusif dévolu à l'État dans la production des normes juridiques et d'autre part par la rédaction de la charte des organisations internationales qui ne fait aucunement la distinction entre les règles constitutionnelles de droit interne et les règles conventionnelles de droit international. Ainsi, l'idée du droit interne des organisations internationales, dégagée d’abord par la jurisprudence du tribunal administratif de la SDN, sera réaffirmée après vingt-cinq d'hésitations doctrinales, à la barre de la CIJ à travers l'avis consultatif sur l'effet des jugements du tribunal administratif des Nations Unies accordant indemnité.

$801 \quad$ Ibid, p.71.

802 Nous suivons ici la démarche utilisée par le Professeur Michel VIRALLY dans son article sur « la valeur juridique des résolutions des organisations internationales " précité. 


\section{A : LA VALEUR JURIDIQUE DES ACTES EDICTES ENTRE ORGANES HIERARCHISES}

Les rapports juridiques entre les organes restreints et les autres organes, particulièrement les organes pléniers peuvent s’analyser en termes d'hiérarchie. De ce fait, l'organe restreint peut être situé dans une position d'infériorité hiérarchique ou encore de supériorité hiérarchique. La valeur juridique des actes édictés par l’organe restreint dépendra de sa situation hiérarchique vis-à-vis des autres organes de l'organisation.

Au regard de l'acte constitutif de l'organisation internationale, les actes édictés par un organe restreint en situation d'infériorité juridique par rapport à l'organe plénier ne peuvent pas produire d'effets contraignants. En raison de son infériorité juridique à l'égard de celui auquel il s'adresse, lauteur de la recommandation est alors hors d'état de conférer force obligatoire à celle-ci. Elle aura la valeur d'une simple proposition dans le cadre d'une procédure de décision où l'organe subordonné est chargé de préparer les délibérations de lorgane supérieur et éventuellement, de rédiger un texte qui ne prendra valeur juridique qu’après avoir été adopté par ce dernier. ${ }^{803}$ Un acte juridique émanant d'un organe restreint et destiné à un organe juridiquement supérieur n’a aucune valeur obligatoire.

Dans cette hypothèse, on peut valablement citer les recommandations faites par le Conseil économique et social à l'Assemblée générale. Aux termes de l'article 62 alinéa 1 de la Charte, «le Conseil économique et social peut faire ou provoquer des études et des rapports sur des questions internationales dans les domaines économique, social, de la culture intellectuelle et de l'éducation, de la santé publique et autres domaines connexes et peut adresser des recommandations sur toutes ces questions à l'Assemblée générale, aux membres de l'organisation et aux institutions spécialisées intéressées...» ${ }^{804}$ Les recommandations adressées par le Conseil économique et social à l'Assemblée générale ne sont pas obligatoires. Pour le Professeur Claude RUCZ, «dans l'ordre interne de lorganisation, l'autorité des recommandations du Conseil est fonction de la place de son destinataire dans la hiérarchie des organes. Les recommandations du Conseil à l'Assemblée générale sont une contribution indispensable à la préparation de ses travaux en ce qui concerne aussi bien les méthodes de travail (documentation, organisation des travaux) que les questions de fond : mais elle laisse intact le pouvoir de décision de l’Assemblée.» ${ }^{805}$

L'Acte constitutif de l'UA montre que les recommandations faites par le Conseil de paix et de sécurité et par la Commission n’ont aucun effet obligatoire à l'égard de la Conférence des Chefs d'État et de gouvernement. Larticle 9 paragraphe 1 alinéa (b) dispose que «les pouvoirs et attributions de la Conférence sont les suivants : [...] recevoir, examiner et prendre les décisions sur les rapports et les recommandations des autres organes de l'Union et prendre des décisions à ce

803 VIRALLY (Michel), " La valeur juridique des recommandations des organisations internationales ", op.cit., p.74 et 75 .

804 Voir l'article 62 de la Charte des Nations Unies.

805 RUCZ (Claude), «Commentaire de l'article 62 de la Charte des Nations Unies », op.cit., p.962. 
sujet ; ${ }^{806}$ Une recommandation émanant du Conseil de paix et de sécurité ou de la Commission n’ont pas pour effet de contraindre la Conférence des Chefs d'État et de gouvernement. Au contraire, en sa qualité d'organe suprême de l'UA, la Conférence examine et approuve les recommandations de ces organes restreints.

En revanche, lorsque l'acte juridique émane d'un organe restreint juridiquement supérieur à l’organe destinataire, sa force obligatoire est impliquée dans cette supériorité qui, sans elle, serait dépourvue de signification. Plus exactement, c'est à l'auteur de l'acte de déterminer luimême, dans certaines limites, le degré d’obligation qu'il entend lui conférer. ${ }^{807} \mathrm{~A}$ titre d'exemple, nous pouvons citer l'article 8 de la Constitution de l'OIT qui dispose que le Directeur général « recevra ses instructions ${ }^{808}$ du Conseil d'administration. ${ }^{809}$ L’organe restreint, le Conseil d'administration est juridiquement supérieur à l'organe auquel est destiné l'acte juridique, le directeur général. Dans ce cas, le caractère obligatoire de l'acte ne saurait être remis en cause ${ }^{810} \mathrm{Il}$ découle du lien de subordination existant entre le Conseil d’administration et le Directeur général.

Le lien hiérarchique entre les organes restreints et les autres organes détermine les effets des actes juridiques des ceux-ci vis-à-vis de ceux-là. Toutefois, entre organes indépendants il en va différemment.

\section{B : LA VALEUR JURIDIQUE DES ACTES EDICTES ENTRE ORGANES INDEPENDANTS}

Dans ce cas, l’organe restreint se situe dans un rapport dégalité avec l'organe auquel est destiné l’acte juridique. ${ }^{811}$

Le Professeur VIRALLY souligne que «la nature même des relations juridiques existant ici entre l'auteur et le destinataire -de l'acte juridique- exclut semble-t-il toute possibilité d’obligation :

806 Voir l'article 9 paragraphe 1 alinéa (b) de l'Acte constitutif de l'UA.

807 VIRALLY (Michel), «La valeur juridique des recommandations des organisations internationales », op.cit., p.72.

808 Le terme instruction utilisé ici dans le cadre de l'OIT ne doit pas être interprété autrement. Il renvoie aux recommandations et aux résolutions prises en tant quactes d'instruction de service dans le cadre d'une administration internationale.

809 Larticle 8 de la Constitution de l'OIT est libellée comme suit : «1. Un Directeur général sera placé à la tête du Bureau internationale du Travail ; il sera désigné par le Conseil d’administration, de qui il recevra ses instructions et vis-à-vis de qui il sera responsable de la bonne marche du Bureau ainsi que de lexécution de toutes tâches qui auront pu lui être confiées.

2. Le Directeur général ou son suppléant assisteront à toutes les séances du Conseil d’administration. »

810 Si le caractère obligatoire de tels actes ne pose pas de problème, un point tout de même essentiel mérite dêtre soulevé. En effet, quelles seront les conséquences de la méconnaissance d'un acte juridique émanant d'un organe supérieur par un organe subordonné ? Pour le Professeur Michel VIRALLY, en cas de méconnaissance d'une recommandation émanant d'un organe supérieur, force est de reconnaître que, dans l'état actuel du droit des organisations internationales aucune sanction n’est prévue. (Voir Michel VIRALLY, dans son article sur « la valeur juridique des recommandations des organisations internationales », déjà cité.)

811 L'exemple type de ce rapport est celui existant entre le Conseil de sécurité et l’Assemblée générale, bien que les membres non permanents du premier soient élus par la seconde. 
ce serait porter atteinte à cette indépendance réciproque affirmée au départ.» ${ }^{812}$ Telle est la situation juridique qui prévaut entre le Conseil de sécurité et l’Assemblée générale des Nations Unies.

En raison de l'indépendance existant entre ces deux organes, les actes juridiques du Conseil de sécurité destinés à l’Assemblée générale n’ont aucune valeur obligatoire. Cependant, ils participent à la collaboration nécessaire entre ces deux organes politiques dans le fonctionnement de lorganisation. Il s'agit par exemple de la participation du Conseil à une décision conjointe avec l'Assemblée générale. ${ }^{813}$ Ces actes sont relatifs à l'admission d'un État à l'ONU (article 4 paragraphe 2 de la Charte), la suspension et l'exclusion des États membres (article 5 et 6 de la Charte), la nomination du Secrétaire général (article 97 in fine de la Charte), la nomination des juges de la CIJ. ${ }^{814}$ Dans tous ces cas - excepté la nomination des juges à la CIJ- le Conseil de sécurité fait une recommandation à l'Assemblée générale. La question qui mérite dêtre posée ici est bien celle de savoir la valeur juridique de cette recommandation du Conseil de sécurité à l'Assemblée générale ? Le Professeur Lino DI QUAL répond à cette interrogation en soulignant que «la recommandation du Conseil ne lie l'Assemblée mais constitue un acte créant une nouvelle situation de droit. ${ }^{815}$ L’Assemblée générale a le pouvoir de refuser d’admettre un État proposé par le Conseil de sécurité. La composition de l'Assemblée générale étant plus large que celle du Conseil, la majorité des membres de l'Assemblée générale peut ne pas s'aligner sur la position du Conseil de sécurité. ${ }^{816}$

Cependant, la CIJ dans son avis consultatif du 3 mars 1950 relatif à l'admission d'un État à l'ONU précise que «la recommandation du Conseil de sécurité est la condition nécessaire pour que l'Assemblée générale puisse exercer son pouvoir de décision.» De ce fait, même si la recommandation du Conseil de sécurité n'a pas un effet obligatoire vis-à-vis de l’Assemblée générale, il faut reconnaître que sans elle, l'Assemblée générale ne peut statuer sur l'admission ou l'exclusion d'un membre de l'organisation. Dans ces conditions, les membres permanents du Conseil de sécurité ont encore la possibilité de bloquer toute décision d’admission par le jeu du véto.

La valeur des actes juridiques des organes restreints édictés dans le cadre du fonctionnement interne des organisations internationales dépend considérablement du rapport hiérarchique avec lorgane auquel il est destiné. Cependant, qu’en est-il de la portée des actes édictés par les organes restreints dans le cadre des relations avec les États membres?

812 VIRALLY (Michel), « La valeur juridique des recommandations des Nations Unies », op.cit., p.75.

813 DI QUAL (Lino), Les effets des résolutions des Nations Unies, LGDJ, Paris, 1967, p.47. Voir aussi en ce sens l'article précité du Professeur Michel VIRALLY à la page 76.

814 En ce qui à trait à la nomination des juges à la CIJ, il faut se référer aux dispositions de l’article 4 et 8 du Statut de cette juridiction. En vérité, l’article 4 du Statut de la CIJ ne prévoit pas la nomination par l’Assemblée générale sur recommandation du Conseil de sécurité. Les membres de la Cour « sont élus par l’Assemblée et par le Conseil de sécurité sur une liste de personnes présentées par les groupes nationaux... ». Cependant, l'article 8 précise que « l’Assemblée générale et le Conseil de sécurité procède indépendamment l'un de l'autre à lélection des membres de le Cour..."

815 DI QUAL (Lino), Les effets des résolutions des Nations Unies, op.cit., p.52.

816 Ibid. 


\section{PARAGRAPHE II : LA PORTEE DES ACTES DES ORGANES RESTREINTS DANS LES RELATIONS AVEC LES ÉTATS}

Contrairement aux actes juridiques édictés dans l'ordre interne des organisations internationales, ceux qui sont édictés dans les relations extérieures, particulièrement dans les relations avec les États posent plus de problèmes juridiques. En vue de donner un début de réponses à ces problèmes, il est nécessaire d'analyser l'effectivité (A) et l'autorité de ces actes dans lordre interne des États membres (B).

\section{A : L'EFFECTIVITE DES ACTES JURIDIQUES DES ORGANES RESTREINTS DANS L'ORDRE INTERNE DES ÉTATS}

Il convient au préalable de donner une approche sémantique au concept d'effectivité en droit internationale public. Il faut le souligner, la doctrine est assez muette sur la question de l'effectivité dans la mesure où elle n’a pas fait l'objet d’abondants développements.

Le Professeur Charles DE VISSCHER, dans son étude consacrée à la question affirme que «la place faite à l'effectivité chez les auteurs est assez déconcertante. La plupart n’en parlent quen termes vagues, comme en passant, parfois simplement par allusion. D’autres relèvent sa présence dans quelques situations, toujours les mêmes, en concevant d’ailleurs de l'imprécision de la notion. Il en est enfin qui se la représentent comme un principe général et autonome, doté d’une autorité normative propre dont les applications couvriraient l'ensemble du droit international.» ${ }^{817}$ Pour la compréhension de notre présente analyse, il est impérieux de donner un contenu, bien entendu, au sens du droit international public, à cette notion.

Le Professeur Charles DE VISSCHER soutient que «s'il n’apparaît pas que l'effectivité soit, au sens propre, un principe du droit international, il est vrai quelle reflète hautement l'esprit de ce droit, du fait qu'elle tend essentiellement, par un recours particulièrement fréquent au «vrai» au « réel», à parer à ses étroitesses formelles, à ses faiblesses les plus notoires, à s’affranchir des représentations ou constructions plus ou moins artificielles pour atteindre dans les voies de léquité, de la praticabilité, de la stabilité, de la finalité, la pleine réalisation de la règle de droit.» ${ }^{818}$ De ce fait, il faut souligner que dans l'ordre du droit international objectif (règle de droit), l'effectivité se caractérise par l'impact, par la pesée directe, souvent impérieuse, du fait sur l'évolution du droit. ${ }^{819}$ Le Professeur Prosper WEIL renforce cette idée en soulignant « la répugnance du système normatif international à accepter une dissociation entre le fait et le droit, de sa réticence à laisser croire que ce qui est ne l'est pas valablement et de sa tendance à décréter ce qui est doit être. Telle est, en effet,

817 DE VISSCHER (Charles), Les effectivités du droit international public, Pédone, Paris, 1967, p.8.

818 Ibid, p.11.

819 Ibid, p.15. 
la signification de l'importance accordée au droit international, bien plus que par tout autre système juridique, à ce qu’on courammant, les « effectivités "»». ${ }^{820}$

Pris dans cette acception, en rapport avec les actes juridiques édictés par les organes restreints destinés aux États, la question de l'effectivité revêt un intérêt juridique considérable. Ici, il s'agit de répondre à la problématique générale des rapports que chaque ordre juridique interne entretient avec les règles d'origine internationale. ${ }^{821}$

Il est bien établi que l'exécution des actes unilatéraux des organisations internationales est par nature décentralisée. ${ }^{822}$ Contrairement à l'État, les organisations internationales ne possèdent pas de mécanismes exécutifs ; l’exécution des décisions revient aux États eux-mêmes. ${ }^{823}$ En d’autres termes, l'effectivité des résolutions dépend du comportement des États agissant en qualité d'agents d'exécution. ${ }^{824}$ Elle dépend entièrement «du bon vouloir des États.» ${ }^{825}$

Généralement des raisons politiques peuvent influencer les États dans l'exécution d'un acte d'un organe restreint. Il s'agit par exemple des circonstances politiques ayant entouré son adoption. Le cas de la résolution 1721 du Conseil de sécurité des Nations Unies en date du $1^{\text {er }}$ novembre 2006 sur la situation en Côte d'Ivoire paraît très édifiant. En effet, comme précédemment indiqué dans les propos de l'Ambassadeur John BOLTON, l’adoption de cette résolution a été marquée par des manœuvres diplomatiques de la France. Mais, il est important de ne pas se méprendre sur le rôle joué par la France au Conseil de sécurité. En général, les États interviennent dans les relations internationales surtout pour défendre leur réputation. Cette thèse a été défendue par le Professeur GUZMAN dans plusieurs travaux en droit international. A ce sujet, il soutient que : « in the absence of other enforcement mechanism, then, a state's commitment is only as strong as its reputation. In entering into an international commitment, a country offers its reputation for living up to its commitment as a form of collateral [...] The point here is that a country reputation has value, and a country will hesitate before compromising that reputation.... ${ }^{826}$

Dans son contenu cette résolution posait le problème du rapport entre une résolution du Conseil de sécurité et la Constitution de l'État de Côte d'Ivoire. Ainsi, l'ex-Président de la République de Côte d'Ivoire, Laurent GBAGBO affirmait que le projet de résolution proposé par la France contient «la grave affirmation selon laquelle les décisions du Conseil de sécurité prévalent

\footnotetext{
820 WEIL (Prosper), « Le droit international en quête de son identité, cours général de droit international public », op.cit., p. 212 .

821 LANFRANCHI (Marie-Pierre), "La valeur juridique en France des résolutions du Conseil de sécurité », AFDI, 1997, p.33.

$822 \quad I b i d$, p.32.

823 Ibid.

824 Ibid, p.33.

825 Ibid.

826 GUZMAN (Andrew T.), «International law: a compliance based theory ", UC Berkeley public law and legal theory working paper, $\mathrm{n}^{\circ} 47$, April 2001, p.27-28.
} 
sur la Constitution ivoirienne et la législation du pays.» ${ }^{827}$ Dès lors, pour lui, «cette proposition posait pour la première fois la double question de la souveraineté des États africains indépendants et de l'égalité de ces États avec les autres États du monde.» ${ }^{828}$

Naturellement, ce point de l'avant projet proposé par la France touchant à la souveraineté de l'État de Côte d'Ivoire n’a pas eu l'adhésion totale ni des autres membres permanents, ni des membres non permanents. ${ }^{829}$

Toutefois dans sa version achevée du $1^{\text {er }}$ novembre 2006, la résolution 1721 contient aussi des violations flagrantes de la Constitution ivoirienne. Le Conseil de sécurité, en sa 5561éance du $1^{\mathrm{er}}$ novembre 2006 adopta la résolution 1721 . Cette résolution souligne que :

"8.le premier ministre, pour l'exécution du mandat mentionné au paragraphe $7 \mathrm{ci}$ dessus, doit disposer de tous les pouvoirs nécessaires, de toutes les ressources, matérielles et humaines requises et une autorité totale et sans entraves, conformément aux recommandations de la CEDEAO en date du 6 octobre 2006, et qu'il doit pouvoir prendre toutes les décisions nécessaires, en toutes matières, en Conseil des ministres ou en Conseil de gouvernement, par ordonnance ou décret- loi.

9. Souligne également que le Premier Ministre, pour l'exécution du mandat mentionné au paragraphe 7 ci-dessus, doit disposer également de toute l'autorité nécessaire sur les Forces de défense et de sécurité de Côte d'Ivoire [...],

13. Demande instamment au Premier Ministre de prendre immédiatement par la voie d’ordonnance qu'il signera dans les conditions énoncées au paragraphe 8 ci-dessus, toutes les mesures appropriées en vue d'accélerer la délivrance des certificats de naissance et de nationalité dans le cadre du processus d'identification dans un esprit d’équité et de transparence ; ${ }^{830}$

Comme on peut le constater, cette résolution est en contradiction avec certaines dispositions de la Constitution ivoirienne. A l'analyse, elle fait du Premier Ministre le titulaire du pouvoir exécutif :

-il a le pouvoir de prendre toutes les décisions nécessaires et en toutes matières, en Conseil des Ministres ou en Conseil de gouvernement, par ordonnance ou décret-loi et il peut signer par ordonnance toutes les mesures appropriées en vue de la délivrance des certificats de nationalité et de naissance dans le cadre du processus d'identification. Ces deux points de la résolution 1721 violent l'article 75 alinéa 1de la Constitution ivoirienne de 2000. Aux termes de cette disposition «Le président de la République peut, pour l'exécution de son mandat, demander à l’Assemblée nationale, l'autorisation de prendre par ordonnance, pendant un délai limité, des mesures qui sont relatif à son l'adoption de la résolution 1721 disponible sur le site internet de la République de Côte d'Ivoire : www.presidence.ci. Ibid.

829 En effet, parmi les membres permanents la Chine et la Russie ont posé leur droit de véto sur l'adoption de ce point de l'avant projet.

830 Voir la résolution 1721 du Conseil de sécurité. 
normalement du domaine de la loi... ${ }^{831}$ De cette disposition, il faut retenir que la Constitution ivoirienne fait du Chef de l'Etat le détenteur exclusif du pouvoir dédiction des ordonnances. La résolution 1721, en conférant un tel pouvoir au Premier Ministre sécarte de la lettre et de l'esprit de la Constitution ivoirienne,

- Pour l'exécution de son mandat, il doit disposer de l'autorité nécessaire sur les Forces de défense et de sécurité. Cet autre point de la résolution 1721 n'est pas conforme à l'article $47 \mathrm{de}$ la Constitution ivoirienne. Cette disposition précise que « Le président de la République est le chef suprême des armées. Il préside le Conseil Supérieur de la défense ".

Au regard des contradictions de la résolution 1721 avec la Constitution ivoirienne, l'exChef de l'État a-t-il saisi le Conseil Constitutionnel dans le cadre d'un avis consultatif. ${ }^{832}$

Lavis rendu sur la question par la haute juridiction constitutionnelle ivoirienne est révélateur sur deux points essentiels. Au niveau de la compétence du Conseil Constitutionnel pour contrôler la constitutionnalité d'une résolution du Conseil de sécurité de l'ONU. En effet, il ressort de la Constitution ivoirienne et de la loi organique sur le Conseil constitutionnel que le contrôle de la constitutionnalité d'une résolution du Conseil de sécurité ne relève pas formellement de sa compétence. ${ }^{833}$ Dans le fond, cet avis consultatif déclare l'inconstitutionnalité de la résolution 1721 du Conseil de sécurité. La haute juridiction constitutionnelle déclare que «cette organisation -les Nations Unies- ne peut donc, sans outrepasser ses pouvoirs, sans commettre un excès de pouvoir ou un détournement de fonction, ni dissoudre, ni modifier, ni même suspendre la Constitution d’un État, ou encore moins placer cet État sous régime de tutelle, ce régime ne pouvant s’appliquer, en vertu de l'article 78 de la Charte, aux pays devenus membres des Nations Unies, les relations entre ceux-ci devant être fondées sur le respect du principe de l'égalité souveraine de tous ses membres.» ${ }^{834}$

831 Voir l'article 75 de la Constitution ivoirienne de 2000.

832 La lettre de saisine du Conseil Constitutionnel par l'ex- Président de la République de Côte d’Ivoire remonte à la date du 27 novembre 2006 et a été enregistrée le même jour sous le n 98 au Secrétariat général du Conseil. Cette lettre qui a été adressé 27 jours après l'adoption de la résolution 1721 montre à quel point le problème juridique qu’elle soulève- la résolution- constitue une question urgente qu'il faut nécessairement élucider.

833 La Constitution de 2000 et la loi organique sur le Conseil constitutionnel ivoirien ne reconnaissent pas formellement au Conseil constitutionnel la compétence d’effectuer le contrôle de conformité des résolutions à la Constitution ivoirienne. Aux termes de ces deux textes, le Conseil constitutionnel est juge de la constitutionnalité des lois, contrôle la régularité des opérations de référendum, statue sur l'éligibilité des candidats aux élections présidentielle et législative ; les contestations relatives à lélection du Président de la République et des députés.

Pour le texte de l'avis du Conseil constitutionnel, il faut se référer aux documents annexes. 
$\mathrm{Au}$ regard de ces considérations juridiques, la résolution 1721 n’a pu être appliquée par l'Etat de Côte d'Ivoire. Ce faisant, l'État ivoirien n'a fait que confirmer la thèse selon laquelle l'effectivité d'un acte unilatéral d'une organisation internationale dépend du bon vouloir de l'État. ${ }^{835}$

Les résolutions du Conseil de paix et de sécurité de l'UA permettent de conforter cette position. Dans la quête de solutions aux crises qui secouent la vie politique des États-membres, le Conseil de paix et de sécurité prend souvent des résolutions. Mais, à l’observation de la réalité politique qui prévaut sur le continent un constat peut être fait. Les différentes résolutions prises par le Conseil de paix et de sécurité sont difficilement mises en application par les États.

En guise d'exemple le cas de la Mauritanie peut être relevé. En effet, un coup d’État perpétré le 3 août 2005 par l'armée a renversé le Président Maaouiya Ould TAYA. Dans une résolution du 4 août, le Conseil de paix et de sécurité condamne fermement ce coup de force et exige le retour rapide à l’ordre constitutionnel, et décide de suspendre la Mauritanie aux activités de l'UA jusqu'au retour de l'ordre constitutionnel dans ce pays. ${ }^{836}$

Dans une autre résolution du 8 septembre 2005, le Conseil prend note des engagements pris par les nouvelles autorités mauritaniennes en vue de faciliter le retour rapide à l'ordre constitutionnel à travers un processus qui culminera avec la tenue délections libres, régulières et transparentes, auxquelles le Président en place a assuré ne pas se présenter ni favoriser de parti. ${ }^{837}$

Malgré ces résolutions du Conseil de paix et de sécurité, un autre coup d’État sera perpétré le 6 août 2008 remettant en cause une transition qui a conduit à des élections civiles, qui pour la première fois a consacré l'avènement d'un régime démocratique.

Les organisations internationales étant dépourvues de moyen de contrainte, il est évident que l'effectivité des résolutions des organes restreints relève de la volonté des États. Quelle est donc l'autorité d'un acte unilatéral d'une organisation internationale dans l'ordre interne des États?

835 D’ailleurs, cette thèse est confirmée dans les relations internationales par la crise du « Moyen -Orient ». Bien entendu, la crise du " Moyen-Orient » désigne habituellement les divers conflits inter ou intra- étatiques (et les situations liées) concernant les pays voisins d'Israël, que ce soit avec ce pays ou entre eux : Israël même, l'Egypte, le Liban, la Syrie, la Jordanie et l'Irak. C’est le sujet qui a donné lieu, depuis 1948 au plus grand nombre de résolutions : 255 sur 1718 (au 18 octobre 2006), soit environ 1 sur 7, et une moyenne de 4 par an. Mais, malgré ce foisonnement des résolutions, cette crise n’a pu connaître de dénouement. Ce manque d’effectivité des différentes résolutions du Conseil de sécurité peut s’expliquer par les circonstances historiques qui ont entouré ce problème. Pour synthétiser, cette crise est née du plan de partage de l'ONU de 1948 qui octroyait 56 $\%$ du territoire aux juifs et $44 \%$ aux palestiniens, puis reconnaissait un statut international à la ville Jérusalem. Evidemment, ce plan de partage sera contesté par le monde Arabe. Mais, aucune résolution ultérieure de l'Assemblée générale et du Conseil de sécurité ne viendra rectifier cette "injustice » faite aux palestiniens. DUJARDIN (Stéphanie), «L'Union africaine : objectifs et moyens de gestions des crises politiques et des conflits armés », op.cit., p.77. Ibid, p.77. 


\section{B : L'AUTORITE DES ACTES JURIDIQUES DES ORGANES RESTREINTS DANS L'ORDRE INTERNE DES ÉTATS}

Réfléchir sur l’autorité des actes juridiques des organes restreints, c'est se demander s'ils sont obligatoires dans lordre interne des États?

La quasi-totalité des auteurs dans la doctrine internationaliste est unanime sur un point : les résolutions n’ont pas en principe d'effet obligatoire à l'égard des États-membres, moins encore vis-à-vis des tiers. ${ }^{838}$ Cette position doctrinale se justifie au regard du principe sacro-saint de la souveraineté de l'État.

La souveraineté de l'État est entendue en droit comme un principe selon lequel l'État n’est soumis à aucune autorité supérieure, ni à aucun pouvoir politique concurrent. ${ }^{839}$ De ce point de vue, l'État est détenteur d'un pouvoir de droit, initial, inconditionné et suprême, ${ }^{840}$ qui induit de ce fait qu'il est le maître absolu de ses décisions politiques et juridiques. C'est auréolé de cet attribut essentiel et fondamental que constitue la souveraineté que les États décident de créer les organisations internationales.

Les États souverains créent les organisations internationales qui en sont des émanations. L'Acte constitutif des organisations internationales qui est un traité révèle bien cette image du créateur et de la créature. ${ }^{841}$ L'État, qui est le créateur de l'organisation internationale, la créature, se trouve dans une position privilégiée par rapport à celle-ci. Les organisations internationales n’ont pas un attribut essentiel de l'État, c'est-à-dire, la souveraineté ou l'impérium qui est le droit de commander et le pouvoir de contraindre. ${ }^{842}$ La conséquence logique est que lorganisation internationale ne peut à travers un organe restreint prendre un acte unilatéral ayant une portée obligatoire dans lordre interne des États.

Une résolution d'un organe restreint d'une organisation internationale doit satisfaire à une condition essentielle pour produire un effet obligatoire dans l'ordre interne des États. Il s'agit comme le souligne le Professeur Benedetto CONFORTI, «des mécanismes propres au droit international classique, c'est-à-dire l'accord et d'autres types similaires, tels que l'acceptation tacite (acquiescement).» ${ }^{843}$ Autrement dit, souligne-t-il, «à défaut d'accord ou d'acquiescement de la part d’un État, la résolution ne produit pas d’effets pour celui-ci.» ${ }^{844}$

\footnotetext{
838 YAO-N'DRE (Paul), Droit des organisations internationales, op.cit., p.87. (Voir aussi en ce sens COMBACAU (Jean), SUR (Serge), Droit international public, Montchrestien, 7e édition,Paris,2006, p. 102 à 107 ; DI QUAL (Lino), Les effets juridiques des résolutions des Nations Unies, LGDJ, 1967, p.107 à 145)

839 LE JEUNE (Pierrick), Introduction au droit des relations internationales, op.cit., p.18.

840 Ibid.

841 KPODAR (Adama), "Le principe de spécialité dans la définition des organisations internationales », RBSJA, $\mathrm{n}^{\circ} 17, \mathrm{p} .74$.

842 Ibid.

843 CONFORTI (Benedetto), "Le rôle de l'accord dans le système des Nations Unies », RCADI, t.142, 1974-II, p.222.

Ibid.
} 
Il est bien établi que les résolutions des organes restreints ne produisent pas d'effets obligatoires dans l'ordre interne des États. Toutefois, il convient de relativiser ce point de vue.

Pour le Professeur YAO-N'DRE, «il est excessif d'affirmer péremptoirement que toutes les résolutions sont dépourvues de force obligatoire. Tout dépend des conditions dans lesquelles une résolution a été adoptée et de son contenu.» ${ }^{845}$

Une résolution du Conseil de sécurité des Nations Unies ou du Conseil de paix et de sécurité de l'UA adoptée à l'unanimité revêt une certaine portée juridique. ${ }^{846}$ Dans une telle hypothèse, la résolution peut produire un effet obligatoire à l'égard des États. Le caractère obligatoire de la résolution découle du vote à l'unanimité des États membres.

Relativement au contenu de la résolution, il faut souligner qu'elle peut être porteuse d' «un droit déclaratoire», c'est-à-dire qu'elle constate l'existence d'une règle obligatoire (traité, coutume, principe général de droit). ${ }^{847}$ Dans ce cas, la résolution tire son caractère obligatoire de la coutume ou du traité. ${ }^{848}$

Le contenu des résolutions du Conseil de sécurité change de caractère juridique suivant que l'on se situe dans le Chapitre VII ou dans le Chapitre VI. ${ }^{849}$ Elles sont obligatoires si elles se fondent sur la Chapitre VII de la Charte. En effet, en vertu du Chapitre VII, le Conseil de sécurité est habilité à prendre des mesures «conformément aux articles 41 et 42 pour maintenir la paix et la sécurité internationales» lorsqu'il «constate l'existence d'une menace contre la paix, d'une rupture de la paix ou d'un acte d'agression». ${ }^{850}$ Mais avant de prendre de telles mesures, le Conseil peut décider des mesures provisoires si bien que le Conseil émet dans le domaine du Chapitre VII trois séries de résolutions. ${ }^{851}$ Ces trois types de résolutions prises au titre du Chapitre VII par le Conseil sont toutes obligatoires. ${ }^{852}$

En revanche, les résolutions qui se fondent sur le Chapitre VI et qui portent sur les modes de règlement pacifique des différends entre les États nont aucun effet obligatoire. Les parties au différend ont une liberté totale pour accepter ou non les résolutions du Conseil de sécurité. 853

Dans la même dynamique, le Professeur Michel VIRALLY invite à prendre en considération «la valeur intrinsèque des résolutions» dans l’appréciation de leur portée dans lordre interne des Etats. ${ }^{854} \mathrm{Il}$ définit la valeur intrinsèque des résolutions comme «un pouvoir spécifique conféré à

845 YAO- N'DRE (Paul), Droit des organisations internationales, Op.cit., p.87.

846 Ibid.

847 DUPUY (René-Jean), « Droit déclaratoire et droit programmatoire, de la coutume sauvage à la Soft law », SFDI, Toulouse, 1974, p.88.

848 Ibid.

849 DI QUAL (Lino), « Les effets juridiques des résolutions des Nations Unies », op.cit.,p.78.

850 Voir l'article 39 de la Charte des Nations Unies.

851 DI QUAL (Lino), « Les effets juridiques des résolutions des Nations Unies », op.cit., p.83.

852 Ibid, p.83 à la page 86.

853 Ibid, p.79.

854 VIRALLY (Michel), « Panorama du droit international contemporain , cours général de droit international public», op.cit., p.200. 
l'organisation internationale par ses membres.» ${ }^{855}$ Les États membres autorisent l'organisation internationale à adopter des actes unilatéraux obligatoires dans leur ordre interne. ${ }^{856}$ Dans la Charte des Nations Unies, ceux-ci ont reconnu au Conseil de sécurité un pouvoir particulier. En effet, par l'article 25 «les membres de l’organisation conviennent d’accepter et d’appliquer les décisions du Conseil de sécurité ...» ${ }^{857}$ De ce fait, les États membres se sont engagés à accepter et à appliquer les décisions du Conseil de sécurité : celles-ci sont donc obligatoires à leur égard. ${ }^{858}$

En outre, un point très important de cette question mérite dêtre souligné. Le principe de la primauté du droit international sur le droit interne largement affirmé et accepté dans la doctrine et par la jurisprudence aujourd'hui conduit absolument à reconsidérer la thèse selon laquelle les

856 On peut citer à titre d’exemple les règlements sanitaires de l'OMS, les standards techniques de l'OACI.

857 Voir l'article 25 de la Charte des Nations Unies.

858 VIRALLY (Michel), « Panorama du droit international contemporain », op.cit., p.200 et 201. 
résolutions des organisations internationales sont dépourvues de force obligatoire. ${ }^{859}$ En vertu de ce principe de supériorité, le droit international s'impose avec force obligatoire à tous les acteurs des relations internationales dont il délimite par ailleurs l'activité juridique. ${ }^{860}$ Le contenu du droit

859 Selon le Professeur DJIENA WEMBOU, « dès l’apparition de l'État-nation en Europe au dix-huitième siècle, il sétait posé la question de la hiérarchie entre le droit international et le droit édicté par les États nationaux. Ce problème, éminemment pratique, a été diversement résolu par les droits étatiques, mais a fait l'objet de disputes homériques au plan doctrinal, entre les tenants du dualisme (pour qui ordre juridique étatique et ordre juridique international constituent deux sphères absolument closes et qui ne communiquent pas) et les différentes catégories de monistes (pour lesquels il n'existe qu'un seul ordre englobant droit international et droit interne, le droit international étant supérieur au droit interne. " (Voir DJIENA WEMBOU, Le droit international dans un monde en mutation, op.cit., p.37.) En effet, la conception dualiste appréhende le droit international et le droit interne comme deux systèmes juridiques séparés, distincts, indépendants et égaux. Ils son impénétrables. En revanche, la thèse moniste dite encore la thèse unitaire est celle qui à l'inverse de la précédente considère le droit international comme un système juridique unique dans lequel les deux catégories de normes sont soumises au principe de la subordination. Mais sur le point de savoir lequel des deux droits doit lemporter sur l'autre, les monistes restent divisés. Pour les uns, c'est le droit interne. Il s'agit là du monisme avec primauté du droit interne. Pour les autres, c'est le droit international. On parle donc du monisme avec primauté du droit international public.

Toutefois, quel est le point de vue de la pratique sur la question ? En réalité, on constate une pratique flottante sur ce point. Tantôt la pratique internationale se prononce contre la thèse dualiste. De ce point de vue, deux exemples peuvent être cités : d'abord, un traité ne sera valable dans lordre international que s'il a été régulièrement conclu dans l'ordre interne, ensuite c'est l'aptitude d'un traité à abroger une loi interne. Tantôt cette pratique se prononce contre la thèse moniste avec primauté du droit international. Dans cette perspective, les règles de compétence et de procédure en vue de la conclusion des traités internationaux sont fixées non pas par le droit international mais par le droit interne. Et, le contentieux international se réduit à un contentieux de la réparation. Aussi, une décision juridictionnelle interne revêtue de l'autorité de la chose jugée ne peut être cassée par une décision internationale. Malgré son caractère flottant, ont peut déceler de la pratique internationale une tendance à la confirmation du monisme avec la primauté du droit international. Cette pratique peut se vérifier à la fois au plan des dispositions constitutionnelles et de la jurisprudence internationale. D’abord au regard des dispositions constitutionnelles, il en existe toute une panoplie:

- La première disposition est la clause affirmant la volonté de l'État de se conformer au droit international. Mais, cette clause reste de peu de secours parce qu'elle ne tranche pas véritablement le problème de la hiérarchie entre le droit international et le droit interne.

- Il y a aussi, la clause conférant la valeur de droit interne au traité. Ainsi, la Constitution des États-Unis consacre en son article 6 la supériorité de certains traités self- exécuting sur les lois antérieures. Mais pour les conflits entre un traité et une loi postérieure, l’on doit se référer à l’intention du législateur.

- La clause consacrant la supériorité du traité sur la loi interne. D’abord sur la loi ordinaire, l’article 87 de la Constitution ivoirien de 2000 consacre clairement la supériorité du traité sur la Constitution. Par ailleurs, l'article 55 de la Constitution française de 1958 dispose que « les accords ou traités régulièrement ratifiés ou approuvés ont dès leur publication une autorité supérieure à celle des lois. » Ensuite sur la loi constitutionnelle, l'article 54 de la Constitution française prescrit qu’en cas d'incompatibilité entre un projet de traité et la Constitution, celle-ci doit être modifiée. Cette modification de la Constitution en vue de ladapter au traité témoigne de la supériorité de celui.

Ensuite au regard de la jurisprudence internationale, le juge affirme de manière constante la supériorité du droit international sur le droit interne. Ainsi, l'avis consultatif de Cour permanente de justice dans l'affaire des communautés gréco-belges (Avis consultatif 31 juillet 1930, Série B, $n^{\circ} 17$, p.13) résume clairement cette position ; " C’est un principe généralement reconnu du droit des gens que dans les rapports entre puissances contractantes d'un traité, les dispositions d’une loi interne ne sauraient prévaloir sur celles d'un traité.» DJIENA WEMBOU (Michel-Cyr), Le droit international dans un monde en mutation, op.cit., p.36. 
international comprend les traités, la coutume, les principes généraux du droit et les résolutions des organisations internationales.

Il est même aujourd'hui admis dans la doctrine et la jurisprudence que les résolutions des organisations internationales peuvent produire des droits à l'égard des citoyens dans l'ordre interne des États. On peut citer ici les résolutions du Conseil de sécurité comportant des sanctions économiques. Celles-ci ne sont certes pas limitées aux stricts rapports interétatiques : en réglementant notamment les relations financières, commerciales, elles intéressent aussi les particuliers dans leurs relations avec l'État ainsi que leurs relations inter se. ${ }^{861}$ La jurisprudence du tribunal de commerce de Nanterre du 31 octobre 1990 permet de justifier cette assertion. Dans cette affaire relative aux effets des mesures d'embargo contre l'Irak, la société Lesieur International demandait notamment au juge de prononcer la résolution d'un contrat de vente conclu avec un acheteur koweitien, ainsi que la restitution des marchandises afférentes au contrat « en raison de l'intervention de la résolution 661 du Conseil de sécurité du 6 août 1990.» Statuant en référé, le tribunal, dans une ordonnance peu motivée a fait droit aux demandes présentées par la société Lesieur. $^{862}$

Les organes restreints des organisations internationales disposent de pouvoirs politiques et juridiques concédés par les membres des organisations internationales. Mais ces pouvoirs ne sont pas absolus, ils comportent des limitations importantes.

861 LANFRANCHI (Marie-Pierre), "La valeur juridique en France des résolutions des Nations Unies », op.cit., p.49.

862 Ibid, p.52. 


\section{TITRE II : LES LIMITES AUX POUVOIRS DES ORGANES RESTREINTS}

Les pouvoirs reconnus aux organes restreints des organisations internationales ne sont pas absolus. Ils sont limités par la pratique de leurs créateurs que sont les États (Chapitre I) et par les organes pléniers en raison de leur primauté fonctionnelle (Chapitre II). 


\section{CHAPITRE I : LES LIMITATIONS DES ORGANES RESTREINTS PAR LES ÉTATS}

Une précision essentielle doit être opérée au préalable. Ici, l'État ne doit pas être considéré spécifiquement comme un membre d'un organe quelconque de l'organisation internationale. Il s'agit de l'État en sa qualité de sujet de droit international doté de la souveraineté et créateur de lorganisation internationale.

Pris dans cette acception, l'État limite substantiellement les pouvoirs des organes restreints. Cette limitation se manifeste au plan politique (Section I) et au plan juridique (Section II). 


\section{SECTION I : LES LIMITATIONS D’ORDRE POLITIQUE}

$\mathrm{Au}$ plan politique, les pouvoirs des organes restreints sont limités par les États. Ces limitations se manifestent par la crise de légitimité (Paragraphe I) et le détournement du mandat des organes restreints (Paragraphe II).

\section{PARAGRAPHE I : LA CRISE DE LEGITIMITE DES POUVOIRS DES ORGANES RESTREINTS}

D’après le dictionnaire des termes juridiques du Professeur CORNU, la légitimité se définit comme la «conformité d'une institution à une norme supérieure juridique ou éthique, ressentie comme fondamentale par la collectivité, qui fait accepter moralement et politiquement l'autorité de cette institution ${ }^{863}$ Généralement, le pouvoir légitime est celui qui est accepté par l’ensemble des gouvernés, du moins par la majorité de ceux-ci. ${ }^{864}$ Lautorité du pouvoir repose sur une base psychologique de consentement. ${ }^{865}$ Au regard de la pratique des États, les pouvoirs reconnus aux organes restreints connaissent une véritable crise de légitimité. Comment se manifeste cette crise de légitimité des pouvoirs des organes restreints ? (A) et quelles en sont les implications (B) ?

\section{A : LES MANIFESTATIONS DE LA CRISE DE LEGITIMITE DES POUVOIRS DES ORGANES RESTREINTS}

Au préalable, un effort de précision s'impose. La crise de légitimité dont il sagit concerne surtout les organes restreints des organisations à caractère politique. Dans cette perspective, le Conseil de sécurité des Nations Unies et le Conseil de paix et de sécurité de l'UA peuvent nous permettre de justifier la crise de légitimité dont il est question dans les organes restreints.

A priori, en raison du traité constitutif qui fonde la création des organisations internationales par les États, les pouvoirs reconnus aux organes restreints ne posent pas de problèmes quant à leur acceptation par ceux-ci. Mais dans les faits, ces pouvoirs connaissent une crise de légitimité. Ainsi, particulièrement les États du tiers monde éprouvent dans leur majorité une véritable paranoïa à légard des organes restreints. ${ }^{866}$ Cette crise de légitimité résulte du contenu de certaines résolutions adoptées par ces organes dans l'optique de la résolution de problèmes politiques dans la société internationale. Le contenu des résolutions émanant des organes restreints suscitent des interrogations quant à leur finalité politique dans les relations internationales.

\footnotetext{
863 Vocabulaire juridique, sous la direction de Gréard CORNU, sixième édition, PUF, Paris, 1996, p.485.

864 DJEDJRO (Mélèdje Francisco), Droit constitutionnel, Editions ABC, Abidjan, 2010, p.25.

865 Ibid.

866 FAYE (André), «Quelle place pour l’Afrique dans un Conseil de sécurité élargi à de nouveaux membres ?»op. cit., p.6.
} 
Les faits politiques survenus en Libye en 2010 et 2011 lors de la révolution dans les États Arabes et en Côte d'Ivoire suite aux contentieux nés des élections présidentielles du 28 octobre 2010 constituent un exemple.

La révolution dans les États arabes débutée à la fin de l’année 2010 et se poursuivant en 2011 a révélé l’approche discutable du Conseil de sécurité des Nations Unies dans la résolution des crises politiques au sein des États membres. Contrairement aux autres États arabes tels que la Tunisie et l'Egypte où les mouvements de contestation populaire se sont déroulés de façon pacifique, en Lybie le mouvement de contestation a été marqué par une opposition armée entre des manifestants et l'armée régulière. ${ }^{867}$ A ce sujet, le Professeur PAYANDEH soutient que, «the situation in Libya has to be viewed within the broader context of the uprising in north africa and the Arabic world that culminate in revolutionary regime overthrows in Tunisia and Egypt and violent protests and conflits in numerous other countries». ${ }^{868}$ La crise Libyenne s'inscrit dans le contexte général du printemps arabe. Mais cette crise a été marquée par une contestation armée du régime en place.Face à une telle situation, l'État libyen devrait-il assister passivement à cette insurrection armée ? A la lumière de la résolution 1973 du Conseil de sécurité, nous essaierons d’analyser l'attitude de l'État libyen.

Le Conseil de sécurité agissant dans le cadre du Chapitre VII de la Charte, se prononce sur cette situation dans sa résolution 1973 du 17 mars 2011 en ces termes :

«...considérant que les attaques systématiques qui ont lieu en Libye contre la population civile peut être assimilée à des crimes contre l'humanité, (...)

exprime sa détermination à assurer la protection des civils et des zones peuplés par des civils et l'acheminement rapide et sans encombre d'une assistance humanitaire, (...)

décide détablir une interdiction de tous les vols dans l'espace aérien de la Libye de manière à protéger les civils, (...)

décide, pour assurer la stricte application de l'embargo sur les armes d'inspecter sur leur territoire, y compris les ports et les aéroports ou en haute mer, les bateaux et les avions se rendant ou provenant de Libye, (...)

Voir à ce sujet l'article de Wissem CHEKKAT paru dans le quotidien algérien « Le quotidien d'Oran », du 15 septembre 2011 et publié dans le quotidien ivoirien «Aujourd'hui », n091 du samedi 05, Dimanche 06 et lundi 07 octobre 2011, intitulé « La symétrie du chaos, vers un nouveau paradigme international ? »p.11. Par ailleurs, l’auteur affirme : « De mémoire de révolutionnaires, on a jamais vu des rebelles armés de fusils d'assaut Gewehr-36 avancer contre les troupes gouvernementales de leur propre pays sous la couverture d'hélicoptères de combats avancé, de drones et de bombardiers étrangers tout en bénéficiant de l'appui de l'artillerie navale, le renseignement de l'imagerie satellitaire et l'encadrement de forces spéciales de pays ayant une longue tradition dans la subversion et le sabotage. C’est pourtant ce qui s'est passé en Libye [...] Ce n'est point un hasard si, au départ des contestations, une aide logistique est mise au profit des insurgés, fussent-ils présentés comme de simples manifestants. » ginia Journal of international law, Volume 52, Number 2, 2012, p. 372. 
décide que le gel des avoirs concernera tous les fonds, les avoirs financiers et les ressources économiques qui sont propriété ou contrôlés directement ou indirectement par les autorités libyennes... » ${ }^{869}$

Le contenu de cette résolution suscite des critiques. L’usage que le Conseil de sécurité fait de ses compétences donne matière à réflexion. Pourquoi le Conseil de sécurité assimile-t-il des manifestants armés à des populations civiles? Au regard des faits, il faut distinguer la population civile de certains manifestants armés. Certes, la réaction du régime de libyen face aux manifestations est fortement critiquable. Comme le souligne le Professeur PAYANDEH, « in Libya protests started in january and february 2011 and were met with immediat violent resistance by Gadhafi regime. At this early stage, the media and several non governmental organizations reported that the government used tanks, machine guns, and snippers against protesters $»{ }^{870}$ Toutefois, il est facile d'identifier des personnes lourdement armées qui se sont attaquées aux édifices publics (tels que les commissariats de police, des camps militaires) parmi les manifestants. Aussi, face à une telle situation de nature à mettre en péril l'intégrité de l'État, les autorités libyennes devraient- elles s'abstenir de toutes réactions? Elles ont réagi en essayant de repousser ce quelles considèrent comme une tentative de déstabilisation du régime politique en place.

Cette résolution du Conseil de sécurité soulève la question de la responsabilité de protéger $^{871}$ des Nations Unies dans les États en crise. Cette notion est clarifiée par Monsieur Peter HILPOLD en ces termes : « Formally, states sovereignty is safeguarded, as the primary responsability for the protection of its people which continues to lies with the state itself. The exception to this rule appears to be, however, far-reaching: Where a population is suffering serious arm, as a result of internal war, insurgency, repression, or state failure, and the state in question is unwilling or

869 Voir la résolution 1973 du Conseil de sécurité de l’ONU.

870 PAYANDEH (Mehrdad), «The United Nations, Military intervention, and regime change in Lybia », in Virginia Journal of international law, Volume 52, Number 2, 2012, p.372.

871 Cette notion de la responsabilité de protéger est née suite à la question posée par l'ex-Secrétaire général des Nations Unies Kofi ANNAN devant l’Assemblée générale en 1999 : « si l'intervention humanitaire constitue effectivement une atteinte indamissible à la souveraineté, comment devons nous réagir [...] devant des violations flagrantes, massives et systématiques des droits de l'homme, qui vont à l'encontre de tous les principessur lesquels notre condition dêtres humains? » Trois ans plus tard, en 2002, apparaît le concept de " responsabilité de protéger » dans le Rapport Evans-Sahnoun, sur le principe et les modalités de la responsabilité de protéger. Il a été produit par la Commission internationale de l'intervention et de la souveraineté des États, créée à l'initiative du Canada et d'un groupe de grandes fondations. Ses conclusions ont été largement reprises par Kofi ANNAN et en 2005 par le groupe de personnalités de haut niveau sur les menaces, les défis et le changement. C'est ainsi qu'en 2005, l'Assemblée générale admis l'existence d'une responsabilité de protéger les populations en cas de génocide, crimes de guerre, purification ethnique et crimes contre l'humanité. Il est à noter que les catastrophes naturelles ne sont pas prises en compte. Le Conseil de sécurité entérine ce principe le 28 avril 2006 dans sa résolution 1674 où il « réaffirme les dispositions des paragraphes 138 et 139 du Document final du Sommet mondial de 2005 relatives à la responsabilité de protéger les populations du génocide, des crimes de guerre, de la purification ethnique et des crimes contre l'humanité ». 
unable to halt or avert it, the principle of non intervention yieds to the international responsability to protect $» .872$

Le principe de la responsabilité de protéger souligne l'importance de la protection des populations civiles dans les États en crise. La responsabilité de protéger repose sur le principe que chaque État a la responsabilité de respecter sa population et de respecter les droits qui lui incombent. C’est seulement dans le cas où l'État faillirait à sa tâche, que la communauté internationale serait dans le devoir d'agir sous légide de la responsabilité de protéger. Mais dans le cas de la crise libyenne, la mise en œuvre de ce principe soulève des interrogations.

Comme le prescrit la résolution 1973, la protection des personnes civiles s'avère indispensable eu égard aux nombreuses pertes en vie humaine que pourrait engendrer la situation politique résultant de la contestation populaire en Libye. Mais, il faut poser la question de savoir si l'intervention en vue de la protection des populations civiles doit servir de prétexte au Conseil de sécurité pour s’attaquer ouvertement à un régime politique ? Cette interrogation est partagée par MEHRDAD qui affirme que « The events in Lybia raise seriouse questions about legality, legitimacy and feasability of pro-democratic military interventions and about the role of intervening states and United Nations (particulary the Security Council) in regime change ${ }^{873}$

Le Conseil de sécurité dans cette résolution décide d'imposer une zone d'exclusion aérienne et le gel des avoirs des autorités libyennes. Une fois de plus, pourquoi le Conseil de sécurité a-t-il écarté la possibilité d'une solution négociée entre les parties au conflit comme le prescrit le chapitre VI de la Charte? Par ailleurs, pourquoi la solution de règlement pacifique préconisée par l'Union Africaine n’a-telle pas été suivie par le Conseil de sécurité ? Selon MEHRDAD Payandeh, «After the military intervention has begun, the African Union high level Committee expresse its general support for resolution 1973, but it also raised concerns regarding military intervention and declared the need for a peaceful solution within african framework ».

En décidant le gel des avoirs des autorités libyennes et l'imposition d'une zone d'exclusion aérienne, le Conseil de sécurité ne donne t-il pas une caution aux mouvements insurrectionnels au sein des États? Cet état de fait conduit à remettre en cause la finalité des pouvoirs que les États ont consentis au Conseil de sécurité. Ce constat semble être confirmé par la position que cet organe a adopté durant la longue crise politique survenue en Côte d'Ivoire.

Le 19 septembre 2002, un mouvement armé se déclenche en Côte d'Ivoire. Le territoire de cet État est divisé en deux parties : la partie nord est occupée par les rebelles et la partie sud est contrôlée par les forces régulières. Aussi, « ayant déterminé que la situation en Côte d’Ivoire continuait de menacer la paix et la sécurité internationales et agissant en vertu du Chapitre VII de la Charte des Nations Unies, le Conseil de sécurité a aux termes de sa résolution 1528 du 27 février p.355. 
2004, décidé de créer l'Opération des Nations Unies en Côte d'Ivoire (ONUCI) pour une période initiale de douze mois à compter du 4 avril 2004. $»^{874}$ Le mandat assigné à cette mission des Nations Unies était principalement le désarmement, la démobilisation et la réinsertion des rebelles. Dans les faits, aussi paradoxale que cela puisse paraître, le désarmement des rebelles n’a pas été effectué par la mission des Nations Unies.

C'est donc en l’absence d'un désarmement des insurgés que les élections présidentielles sont organisées en Côte d'Ivoire en octobre 2010. Après le deuxième tour, la Commission électorale indépendante déclare le Candidat Alassane Ouattara vainqueur avec 54,10\% des voix. Quant au Conseil constitutionnel, il donne le candidat Laurent Gbagbo vainqueur avec 51,10\% des voix. Aux termes de larticle 94 alinéa 4 de la Constitution ivoirienne «le Conseil constitutionnel proclame les résultats définitifs des élections présidentielles.» ${ }^{875}$ Ce résultat donné par le Conseil constitutionnel ivoirien est contesté par le candidat de lopposition. Face à cette divergence entre le Conseil constitutionnel et la Commission électorale indépendante, il est nécessaire de chercher à comprendre la procédure électorale mise en place en Côte d'Ivoire. En effet, la procédure légale prévoit l'intervention de trois organes de nature différente : la Commission électorale indépendante, le Représentant spécial du Secrétaire général de l’ONU, le Conseil constitutionnel. ${ }^{876}$ Chaque institution a une fonction spécifique et intervient successivement. La Constitution ivoirienne confère au Conseil constitutionnel, outre ses attributions en matière de contrôle de constitutionnalité des lois et des accords internationaux, la maitrise de toutes élections. Il est même en droit « d’ordonner l'arrêt des opérations électorales ou de décider la suspension de la proclamation des résultats en cas de circonstances graves ». ${ }^{877} \mathrm{Il}$ statue "sur les contestations relatives à lélection du Président de la République » et seul il est compétent pour «proclamer les résultats définitifs des élections présidentielles $»{ }^{878}$ La disposition est claire et ne souffre aucune interprétation. Le Conseil constitutionnel est donc l'ultime organe intervenant dans l'élection présidentielle, ce qui exprime la pleine souveraineté de l'Etat ivoirien. ${ }^{879}$

La Commission Electorale Indépendante est d'une toute autre nature. Elle a été créée par une loi ordinaire du 09/01/2001, intégrée dans le code électoral afin de favoriser "l'organisation délections transparentes ». Elle est compétente selon l’article 59 du code électoral pour réguler le scrutin (opérations de collecte) et annoncer « les résultats provisoires du scrutin, en présence des représentants des candidats $»{ }^{880}$

Entre ces deux institutions s'intercale en vertu d'un accord international de 2005 ( Prétoria), destiné à rassurer l'opposition l’ex- Président Gbagbo, le Représentant spécial de l'ONU.

874 Voir la résolution 1528 du Conseil de sécurité de l’ONU.

875 Voir l'article 94 de la Constitution ivoirienne de 2000.

876 CHARVIN (Robert), Côte d'Ivoire, la bataille de la seconde indépendance, L'Harmattan,Paris, 2O11, p.97.

877 Voir l'article 38 de la Constitution ivoirienne d'août 2000.

878 Voir les articles 91 et 63 du code électoral ivoirien.

879 CHARVIN (Robert), Côte d'Ivoire, la bataille pour la seconde indépendance, op.cit., p.98.

880 Ibid. 
Le paragraphe 6 de la résolution 1765 du Conseil de sécurité définit la mission du Représentant spécial en ces termes : «Le Représentant spéciale du Secrétaire général en Côte d’Ivoire certifiera que tous les stades du processus électoral fourniront toutes les garanties nécessaires pour la tenue délections présidentielles et législatives ouvertes, libres et transparentes, conformément aux normes internationales ". De ce fait, il se borne à l'évaluation des procédures et autres modes opératoires et ce au regard des normes internationales pour les élections démocratiques. ${ }^{881}$ La certification du scrutin est à distinguer de celle de la validation, laquelle revient exclusivement ou à part entière au Conseil constitutionnel, juge de lélection présidentielle. ${ }^{882}$ Mais dans les faits, le Président de la Commission électorale indépendante proclame les résultats des éléctions déclarant vainqueur Alassane OUATTARA par 2483164 voix soit 54, 10\% contre 2107055 soit 45, 90 \% pour Laurent GBAGBO. Le représentant spécial des Nations Unies ajoute que les résultats proclamés sont conformes à ceux certifiés par l'ONUCI. ${ }^{883}$

Il naît donc un contentieux électoral émaillé de violences graves du fait des affrontements entre les forces armées régulières et les insurgés restés fidèles au candidat Alassane Ouattara.

C’est dans ce contexte que le Conseil de sécurité de l'ONU dans sa résolution 1975 du 30 mars 2011 se saisit de la question en vertu du Chapitre VII de la Charte. ${ }^{884}$ Dans cette résolution, le Conseil de sécurité reconnait les résultats publiés par la Commission électorale indépendante en lieu et place des résultats définitifs publiés par le Conseil constitutionnel ivoirien et demande aux forces des Nations Unies en Côte d'Ivoire de mettre tout en œuvre pour « détruire les armes lourdes utilisées par l’armée régulière contre les populations civiles. » Quant au Conseil de paix et de sécurité de l'UA, dans son communiqué de sa $259^{e}$ réunion tenue le 30 janvier 2011, il reconnaît aussi les résultats de la Commission électorale indépendante au détriment de celui du Conseil constitutionnel.

$\mathrm{Au}$ regard de ce qui vient dêtre dit, une question mérite dêtre posée : les résolutions prises tant par le Conseil de sécurité de l'ONU que par le Conseil de paix et de sécurité de l'UA dans la crise post électorale ivoirienne sont elle conformes à la volonté politique des États membres exprimée dans les Actes constitutifs de ces organisations internationales?

Aussi bien la Charte des Nations Unies que l'Acte constitutif de l'UA préconisent le règlement pacifique des conflits. Larticle 2 alinéa 3 de la Charte des Nations Unies dispose que «les membres de l'organisation règlent leurs différends internationaux par des moyens pacifiques, de telle manière que la paix et la sécurité internationales ainsi que la justice ne soient pas mises en danger.» ${ }^{85}$ Larticle 4 paragraphe (e) de l'Acte constitutif de l'UA s'oriente dans le même sens que la Charte de l'ONU en ces termes : «l'UA fonctionne conformément aux principes suivants :... 
Règlement pacifique des conflits entre les États membres de l'Union par les moyens appropriés qui peuvent être décidés par la Conférence de l'Union.» ${ }^{886}$ Dans le contexte d'un contentieux électoral, ne serait-il pas logique et conforme à la Charte des Nations Unies et à l'Acte constitutif de l'UA que le Conseil de sécurité et le Conseil de paix et de sécurité recherchent une solution pacifique à cette crise?

Parmi les principes énumérés par l’article 4 de l’Acte constitutif de l'UA, le paragraphe (p) mentionne la "condamnation et le rejet des changements anti-constitutionnels de gouvernement.» ${ }^{887}$ Cette disposition peut être interprétée de plusieurs façons. Mais dans tous les cas, elle accorde une primauté à la Constitution et aux institutions des États membres. Tout changement de gouvernement dans les États membres de l'UA doit se faire dans le cadre prévu et défini par la Constitution des États. Ainsi, dans la crise post électorale ivoirienne, le Conseil de paix et de sécurité devrait trouver une solution qui s'inscrit dans le respect des institutions d'un État membre. En rejetant les résultats donnés par le Conseil constitutionnel ivoirien au profit de ceux de la Commission électorale indépendante, manifestement le Conseil de paix et de sécurité de l'UA a agit en violation de l'article 4 paragraphe (p) de l'Acte constitutif de l'UA. Dans ces conditions, le Conseil de paix et de sécurité, ce nouvel organe mis en place par les fondateurs de l'UA, peut-il encore jouir de la crédibilité des États membres dans sa mission de résolutions des conflits?

L'approche choisie par le Conseil de sécurité des Nations Unies et le Conseil de paix et de sécurité de l'UA dans la résolution des conflits politiques soulèvent une crise de légitimité des pouvoirs qui leur sont reconnus par les États en tant qu’organes restreints. Mais, quelles sont les implications de cette crise de légitimité des pouvoirs des organes restreints?

\section{B : LES IMPLICATIONS DE LA CRISE DE LEGITIMITE DES POUVOIRS DES ORGANES RESTREINTS}

La crise de légitimité que les pouvoirs du Conseil de sécurité des Nations Unies et du Conseil de paix et de sécurité de l'UA connaissent a nécessairement des implications politiques sur ces organes eux-mêmes. Elles se perçoivent au niveau de la remise en cause de la légitimité de ces organes par la majorité des États. En clair, il s’agit d’une crise de légitimité des pouvoirs qui aboutit à une perte de légitimité des organes restreints. C'est donc à juste titre que le Professeur Elisabeth ZOLLER affirme qu' «en tant qu'expression d'une certaine communauté d'intérêts, l'organisation internationale doit s'appuyer sur la confiance des États qui l'ont créée. Les organes de l'organisation internationale ne peuvent fonctionner efficacement que s'ils ont la confiance des États dont ils émanent.» ${ }^{88}$ Or, compte tenu de la crise de légitimité qui «secoue» les pouvoirs du Conseil de

\footnotetext{
886 Voir l'article 4 paragraphe (e) de l'Acte constitutif de l'UA.

887 Voir l'article 4 paragraphe (p) de l'Acte constitutif de l'UA.

888 ZOLLER (Elisabeth), La bonne foi en droit international public, Pedone, Paris, 1977, p.183.
} 
sécurité et du Conseil de paix et de sécurité, la confiance qui devrait exister entre les États et ces organes tend à s'estomper.

Ainsi que nous l'avons souligné, les États du tiers monde éprouvent une certaine paranoïa vis-à-vis des organes restreints. Cette situation est amplement justifiée par l'interprétation du contenu des résolutions adoptées au sein de ces organes par les États. Avec les résolutions 1973 du Conseil de sécurité sur la crise libyenne et la résolution 1975 sur le conflit post électoral en Côte d'Ivoire, il a été donné de constater une interprétation tendancieuse par la France, les États-Unis et la Grande-Bretagne du contenu de ces actes. D’abord, relativement à la résolution 1973, l’un de ses points les importants concerne l'imposition «d'une zone d'exclusion aérienne» à la Lybie par le Conseil de sécurité. Mais concrètement, que signifie cette injonction ? La résolution 1973 apporte une réponse claire à cette interrogation. Elle précise en son paragraphe 6 qu'il s'agit par la notion de zone d'exclusion aérienne « d'interdire tous les vols dans l'espace aérien de la Jamahiriya arabe libyenne afin d'aider à protéger les civils.» ${ }^{889}$ Mais, entre le contenu de la résolution 1973 et l'interprétation faite par les États-Unis, la France et la Grande-Bretagne il y a un grand fossé. Ces grandes puissances, membres permanents du Conseil de sécurité ont substitué leur interprétation personnelle à celle émanant de la lettre et de l'esprit de cette résolution. De fait, ces grandes puissances ont interprété cette résolution à travers leurs agissements comme la neutralisation par la force armée de toute la flotte militaire aérienne de l’armée régulière libyenne. ${ }^{890}$

Cette interprétation de la résolution 1973 par les grandes puissances pose évidemment des problèmes: pourquoi ne s'est-elle pas faite en se fondant sur le sens naturel et ordinaire des dispositions contenues dans cette résolution? ${ }^{891}$ Larticle 2 alinéa 4 de la Charte des Nations Unies dispose que «les membres de l'organisation s'abstiennent, dans leurs relations internationales, de recourir à la menace ou à l'emploi de la force, soit contre l'intégrité territoriale ou l'indépendance politique de tout État, soit de toute manière incompatible avec les buts des Nations Unies.» ${ }^{892}$ L'interprétation des membres permanents du Conseil de sécurité devrait être compatible avec cette disposition de la Charte. En d'autres termes, ils auraient pu s'abstenir dans la mise en œuvre de la résolution 1973 de tout recours à la force, pour rechercher un règlement politique de la crise.

Quant à la résolution 1975 adoptée par le Conseil de sécurité sur la crise post électorale en Côte d'Ivoire d’octobre 2010, l'usage qui en a été fait par les grandes puissances, particulièrement la France est de nature à discréditer cet organe restreint. Dans cette résolution, « réaffirmant qu’il souhaite vivement que la crise post électorale en Côte d'Ivoire soit réglée de manière pacifique, et qu’lle exige une solution politique globale qui préserve la démocratie et la paix et favorise la

889 Voir le paragraphe 6 de la résolution 1973 du Conseil de sécurité sur la Libye.

890 Dans les faits, les forces de l'Organisation du traité de l'Atlantique du Nord (OTAN) dirigées par les France, la Grande-Bretagne et les États Unis ont procédé à des frappes militaires visant toute la flotte aérienne de l'armée régulière libyenne. Ces États ont estimé agir dans le cadre de la résolution 1973 du Conseil de sécurité. 
réconciliation durable de tous les ivoiriens...», ${ }^{893}$ le Conseil de sécurité exclut tout recours à la force dans la résolution de cette crise. Dans le cadre d'un conflit post électoral, cette voie de règlement pacifique préconisée par la résolution 1973 semblait la mieux indiquée. Dans cette perspective, la recherche de la vérité sortie des urnes était indispensable pour la crédibilité du Conseil de sécurité. ${ }^{894}$

Mais, cette volonté de régler pacifiquement la crise ivoirienne est fortement contrariée par le paragraphe 6 de la résolution 1975 qui «autorise l'ONUCI, dans le cadre de l'exécution de son mandat, à utiliser tous les moyens nécessaires pour s'acquitter de la tâche qui lui incombe de protéger les civils menacés d'actes de violence physique imminente, dans la limite de ses capacités et dans ses zones de déploiement, y compris l'utilisation d'armes lourdes contre les populations civiles...» ${ }^{895}$ C'est donc sur le fondement de cette disposition que les forces des Nations Unies soutenues par les forces françaises ont pris la décision d'intervenir militairement dans le conflit post électoral. Dans les faits, cette intervention militaire s'est matérialisée par la destruction de l'armement détenue par l'armée régulière.

Au regard de l'intervention militaire des forces des Nations Unies en Côte d'Ivoire suite à la résolution 1975 du Conseil de sécurité, plusieurs observations peuvent être faites. Les forces des Nations Unies se sont écartées de la lettre et de l’esprit de la résolution 1975. En intervenant exclusivement pour détruire les armes détenues par l'armée régulière de Côte d'Ivoire, les forces des Nations Unies n’ont pas exécuté de manière impartiale leur mandat comme le souligne la résolution 1975. De ce fait, les forces des Nations Unies se sont érigées en partie au conflit. Ce qui laisse penser que «les soldats du maintien de la paix» ont été utilisés pour devenir un instrument de changement de régime en Côte d'Ivoire. ${ }^{896}$

Il convient de noter que les pouvoirs des organes restreints sont limités au plan politique par une crise de légitimité. Mais, à celle-ci s'ajoutent d'autres difficultés politiques qui rendent difficiles la mise en ouvre du mandat des organes restreints.

\section{PARAGRAPHE II : LES DIFFICULTES POLITIQUES LIEES AU FONCTIONNEMENT DES ORGANES RESTREINTS}

Dans les organisations internationales, certaines difficultés politiques entravent le fonctionnement des organes restreints. Celles-ci sont essentiellement de deux ordres : ce sont

\footnotetext{
893 Voir la résolution 1973 du Conseil de sécurité des Nations Unies.

894 A ce sujet, l'une des parties au conflit post électoral ivoirien, le candidat Laurent GBAGBO, avait proposé le recomptage des voix pour la manifestation de la vérité des urnes.

895 Voir la résolution 1973 du Conseil de sécurité des Nations Unies. L'ONUCI est le nom donné aux forces des Nations Unies en Côte d'Ivoire.

896 Il est important de souligner que cette intervention des forces armées des Nations Unies soutenues par les forces françaises a aboutit à l'arrestation le 11 avril 2011 de Monsieur Laurent GBAGBO qui avait été déjà déclaré vainqueur par la Conseil constitutionnel ivoirien.
} 
essentiellement les impératifs des groupes régionaux (A) et le détournement du mandat des organes restreints (B).

\section{A : LES IMPERATIFS DES GROUPES REGIONAUX ÉTATIQUES}

On le sait, l'un des critères qui fondent la composition des organes restreints est la répartition géographique équitable. ${ }^{897}$ Cependant, les groupes régionaux imposent des impératifs aux États désignés pour les représenter dans les organes restreints. Cette pratique est de nature à rendent difficile le fonctionnement de ces organes.

L'emprise croissante des groupes ${ }^{898}$ a une incidence fâcheuse sur le fonctionnement interne des organes restreints. Ses conséquences sont plus remarquables sur le processus de vote majoritaire. ${ }^{899}$ Selon le Professeur Michel VIRALLY, «avec le système majoritaire, les choses changent. La prise d'une décision suppose la réunion d'un certain nombre de voix qu'il faut mobiliser [...] En outre, l’opposition à une décision exige aussi la mobilisation d'une pluralité de votes et, par conséquent, un effort de même type.» ${ }^{900}$ Il s'ensuit que dans le processus de vote à la majorité, les différents groupes géographiques sont contraints de procéder à des consultations officieuses avant le vote. Ces consultations officieuses rendent difficile le fonctionnement interne des organes restreints. Au Conseil de sécurité des Nations Unies, il n’est pas contraire à l'esprit de la Charte qu’un État membre s'exprime à la fois en son nom propre et au nom de la zone géographique à laquelle il appartient ni qu'il associe aux travaux du Conseil les efforts en matière de paix et de sécurité menés par les organismes régionaux dont il fait partie. ${ }^{901}$

Dans les faits, cette pesanteur des groupes régionaux entraîne un comportement monolithique chez les États africains membres du Conseil de sécurité. ${ }^{902}$ Choisis par leur groupe pour être élus au Conseil, ils sont étroitement soumis à son contrôle et mandatés par lui. En conséquence, ils doivent consulter en permanence, sinon toujours les quelques cinquante États composant le groupe africain..$^{903}$

Ces consultations officieuses ont des répercussions sur les travaux de cet organe. Pour le Professeur Marie-Claude SMOUTS, s'il est vrai que « rien dans la Charte n’autorise et n’interdit, la stricte discipline de groupe [...] L’objection majeure tient plutôt à la diplomatie multilatérale par

899 A ce sujet, les Professeurs Laurent ZANG et David SINOU soulignent que « des trois modes de prises de décision qu'on rencontre dans les organisations internationales -la règle de l'unanimité, celle de la majorité et le consensus- seul, le second est favorable à l'apparition des groupes. »(« Dynamique des groupes au sein de l'OUA et unité africaine» in Maurice KAMTO, Jean-Manuel PONDI, Laurent ZANG, L'OUA : rétrospective et perspectives africaines, p.139.)

900 VIRALLY (Michel), Lorganisation mondiale, op.cit., p.281.

901 SMOUTS (Marie-Claude), «Réflexions sur les méthodes du Conseil de sécurité », op.cit., p.605.

902 Ibid, p.608.

903 Ibid. 
l'excès de rigidité de quelques uns. Faute d'avoir un minimum de latitude dans la discussion, les États africains donnent le sentiment d'avoir à négocier avec d’autres interlocuteurs que ceux qui siègent avec eux, avec des partenaires absents et inconnus. „ ${ }^{904} \quad$ Manifestement, cette situation est de nature à entraîner des blocages dans la procédure de prise de décision au sein du Conseil de sécurité.

Or, le Conseil est un organe conçu pour agir efficacement et rapidement face aux situations d'urgence. Dans ces conditions, il est légitime de s'interroger si ces mêmes obstacles ne vont pas entraver le fonctionnement du Conseil de paix et de sécurité de l'UA ? Calqué sur le modèle du Conseil de sécurité de l'ONU, il est composé des États issus des cinq régions géographiques du continent africain.

Mais à la différence du Conseil de sécurité de l'ONU, aux termes de l'article 8 paragraphe 13 de son Protocole «les décisions du Conseil de paix et de sécurité sont généralement guidées par le principe du consensus.» ${ }^{95}$ Le consensus se définissant comme une modalité de prise de décision qui exclut tout recours au vote, les fondateurs de l'UA ont sans aucun doute voulu épargner certains blocages inhérents au vote majoritaire à cet organe mis en place pour régler promptement les conflits en Afrique. Mais, il est important de souligner que l'article 8 du Protocole dispose qu' «à défaut du consensus, le Conseil de paix et de sécurité adopte ses décisions sur les questions de procédure à la majorité simple, tandis que les décisions sur toutes les autres questions sont prises à la majorité des deux tiers de ses membres votants.» ${ }^{906}$ Dans l'esprit des fondateurs de l'UA, le recours au vote majoritaire entraînant des consultations informelles n'est possible que lorsque le consensus n’a pas été recueilli entre les États membres sur une question donnée.

En revanche, les grandes puissances qui sont membres des organes restreints ne sont pas liées par les impératifs des groupes régionaux. La question d'un mandat collectif ne se pose donc pas au niveau des grandes puissances. Ainsi, au Conseil de sécurité des Nations Unies, la France et la Grande-Bretagne ont repoussé l'idée d'un double mandat : un pour leur État, un pour la Communauté économique européenne. ${ }^{907}$

Toutefois, les grandes puissances tiennent souvent des consultations dites «officieuses» avant les réunions proprement dites du Conseil de sécurité. C’est au cours de ces consultations que la plupart des décisions du Conseil sont formellement arrêtées avant la discussion avec les autres membres non permanents. ${ }^{908}$ Ces consultations officieuses entre les grandes puissances entraînent une opacité dans le fonctionnement du Conseil de sécurité. Cette opacité du fonctionnement du Conseil de sécurité explique les difficultés rencontrées par les petits États et même les États non

\footnotetext{
$904 \quad$ Ibid, p.609.

905 Voir l'article 8 paragraphe 13 du Protocole relatif à la création du Conseil de paix et de sécurité de l'UA

906 Ibid.

907 SMOUTS (Marie-Claude), «Réflexions sur les méthodes de travail du Conseil de sécurité », op.cit., p.606.

908 FAYE (André), «Quelle place pour l’Afrique dans un Conseil de sécurité élargi à de nouveaux membres ? " op.cit., p.8.
} 
membres à pénétrer le cercle très fermé des grandes puissances préalablement à toute prise de décision. ${ }^{909}$

Les exigences découlant des groupes régionaux et les consultations officieuses entre les grandes puissances constituent des obstacles politiques au fonctionnement des organes restreints. Mais, ces obstacles sont accentués par la pratique de la délégation de compétence des organes restreints par les États.

\section{B : LA PRATIQUE DE LA DELEGATION DE COMPETENCE DES ORGANES RESTREINTS PAR LES ÉTATS}

Ainsi que nous l'avons souligné, les organes restreints jouissent de compétences qui leur sont reconnues et dévolues par les États membres. A ce titre, les membres de ces organes agissent au nom et pour le compte de l'ensemble des États. Cependant par la pratique de la délégation de compétence, les membres des organes restreints font exécuter leur mandat par d'autres acteurs extérieurs à l'organisation.

Dans les faits, les organes restreints des organisations internationales à caractère politique offrent des illustrations de la pratique de délégation de compétence. Ainsi, le Conseil de sécurité applique la technique de la délégation de compétence confiant à certaines grandes puissances la mission d'agir militairement pour le compte des Nations Unies. ${ }^{910}$ Dans la crise libyenne de 2011, la résolution 1973 du Conseil de sécurité «autorise les États membres qui ont adressé au Secrétaire général une demande de notification à cet effet et agissant à titre national ou dans le cadre d’arrangement régionaux et en coopération avec le Secrétaire général, à prendre toutes les mesures nécessaires, [...] pour protéger les populations et les zones civiles menacées d'attaque en Jamahiriya arabe libyenne, y compris Benghazi...» ${ }^{911}$ Sur le fondement de cette résolution l'Organisation du traité de l'Atlantique du Nord (OTAN) s'est engagée militairement dans cette crise politique. Mais au regard des dispositions de la Charte des Nations Unies, le Conseil de sécurité n’a-t-il pas insidieusement procédé à une délégation de compétence?

Comme on le voit dans cette résolution, l’objectif de l'opération est humanitaire : il s'agit de la protection des populations civiles. Et, le Secrétaire général est au centre de cette opération

909 Certaines solutions ont été proposées par les petits États pour rendre plus transparent les travaux du Conseil de sécurité. Ces États qui s'estiment marginalisés du fait du caractère officieux des consultations entre les grandes puissances souhaitent que l'ordre du jour de ces consultations soit distribué. Dans ce cas, ces consultations deviendraient des pourparlers avant la discussion et l'adoption des projets de résolutions. Cependant, si cette proposition présente des avantages, notamment celui d'intéresser le plus grand nombre d'États à la recherche de solutions à un problème, elle ne manque pas d'inconvénients. En effet, les situations de crise demandent parfois des réponses et des mesures rapides. Ce qui serait impossible lorsque la consultation est trop longue.

910 CHARVIN (Robert), Relations internationales, droit et mondialisation, un monde en sens unique, L'Harmattan, Paris, 2000, p.151.

911 Voir la résolution 1973 du Conseil de sécurité. 
qui demeure sous le contrôle politique des Nations Unies. ${ }^{912}$ Mais, il faudrait toutefois relever que la coordination établit avec le Secrétaire général par la résolution 1973 est en deçà des dispositions pertinentes du Chapitre VII de la Charte. ${ }^{913}$ Dans le cadre de l'ONU, le recours à la force est subordonné à une série de conditions prévue par le Chapitre VII de la Charte. Selon l'article 42 de la Charte «si le Conseil de sécurité estime que les mesures prévues à l’article 41 seraient inadéquates ou qu'elles se sont révélées comme telles, il peut entreprendre, au moyen des forces aériennes, navales ou terrestres, toute action qu'il juge nécessaire au maintien ou au rétablissement de la paix et la sécurité internationales. Cette action peut comprendre des démonstrations, des mesures de blocus et d'autres opérations exécutées par les forces aériennes, navales ou terrestres des membres des Nations Unies.» ${ }^{914}$ Cette disposition autorise expressément le Conseil de sécurité à recourir à la force militaire lorsque les circonstances l'exigent. Dans la crise libyenne le Conseil était fondé à recourir à la force au regard des conséquences des affrontements armés sur les populations civiles. Toutefois, ce recours à la force est encadré dans les limites établis par le Chapitre VII.

Aux termes de larticle 43 de la Charte «tous les membres des Nations Unies, afin de contribuer au maintien de la paix et de la sécurité internationales, s'engagent à mettre à la disposition du Conseil de sécurité, sur son invitation et conformément à un accord spécial ou des accords spéciaux, les forces armées, l'assistance et les facilités, y compris le droit de passage, nécessaires au maintien de la paix et de la sécurité internationale [... ] Laccord ou les accords seront négociés aussitôt que possible, sur l'initiative du Conseil de sécurité. Ils seront conclus entre le Conseil de sécurité et des membres de l’organisation, ou entre le Conseil de sécurité et des groupes de membres de lorganisation, et devront être ratifiés par les États signataires selon leurs règles constitutionnelles respectives.» ${ }^{915} \mathrm{Il}$ ressort de cette disposition que pour le recours à la force armée, les États membres doivent mettre leurs forces armées à la disposition du Conseil de sécurité par un accord spécial ratifié selon leurs droits internes. Or, dans la résolution 1973 le Conseil de sécurité «autorise les États membres qui ont adressé au Secrétaire général une notification à cet effet et agissent à titre national ou dans le cadre d’organismes ou d’arrangements régionaux [...] à prendre toutes les mesures nécessaires [...] pour protéger les populations et les zones civiles menacées d'attaque en Jamahiriya arabe libyenne...» Manifestement, cette résolution ne mentionne pas un quelconque accord spécial conclut entre les États membres et le Conseil de sécurité. Elle autorise tout simplement les États membres à agir dans le cadre des organismes régionaux. Dans les faits, aucun accord spécial n'a été conclu entre les États membres de l'Organisation du traité de l'atlantique du nord et le Conseil de sécurité. ${ }^{916}$ Le Conseil de sécurité a délégué sans fondement juridique sa

912 DJIENA WEMBOU (Michel-Cyr), Le droit international dans un monde en mutation, op.cit., p.116.

913 Ibid.

914 Voir l'article 42 de la Charte des Nations Unies.

915 Voir l'article 43 de la Charte des Nations Unies.

916 L'Organisation du traité de l'atlantique du nord s'est officiellement engagée dans le conflit libyen le 31 mars $2 \mathrm{O} 11$. 
responsabilité, en violation des articles 42 et 43 du Chapitre VII de la Charte. Normalement, «dans toute action coercitive internationale ; c’est l'ONU qui doit définir le mandat, c'est l'organisation qui doit suivre et superviser son exécution ; et c'est elle qui doit décider quand le mandat est achevé.» ${ }^{17}$

Aucun article de la Charte n’autorise le Conseil de sécurité à déléguer ses pouvoirs spécifiques qu'il détient dans le domaine du maintien de la paix et de la sécurité internationales, et qui lui ont été délégués par tous les États afin d’assurer l'action rapide et efficace de l'organisation. ${ }^{918}$ Seul un retour aux mécanismes originels de la Charte permettra de restaurer la crédibilité du Conseil de sécurité, et empêchera par là-même que la décision de recourir à la force dans les relations internationales ne devienne une arme juridique redoutable aux mains de certains États qui dominent le Conseil de sécurité. ${ }^{919}$

Au plan politique les pouvoirs des organes restreints demeurent fortement limités. Mais ses limites ne s'arrêtent pas au niveau politique. Il y a aussi des limitations juridiques qu'il convient maintenant d'analyser.

\section{SECTION II : LES LIMITATIONS D’ORDRE JURIDIQUE}

Au plan juridique, l'État, géniteur de l'organisation internationale impose des limites aux organes restreints à travers le contenu des Chartes constitutives (paragraphe I). Mais en dehors de ces limitations contenues dans les Chartes constitutives, il y a celles qui sont souhaitables pour les États. Il s'agit particulièrement du contrôle de la constitutionnalité des actes du Conseil de sécurité de l'ONU (paragraphe II).

\section{PARAGRAPHE I : LES LIMITATIONS IMPOSEES PAR LES ÉTATS A TRAVERS LES CHARTES CONSTITUTIVES}

Les États dotent les oganisations internationales d'une Charte constitutive. Mais l'analyse des Chartes constitutives révèlent qu'elles limitent les pouvoirs des organes restreints. De fait, paradoxalement, l'extension considérable de leurs pouvoirs dans les Chartes constitutives porte atteinte à leur efficacité (A). En outre, les privilèges fonctionnels octroyés par les grandes puissances dans les Chartes constitutives réduisent les pouvoirs des organes restreints (B).

\section{A : L'EXTENSION CONSIDERABLE DES POUVOIRS DES ORGANES RESTREINTS}

En principe, les pouvoirs d'un organe restreint sont orientés essentiellement vers l'efficacité de lorganisation. Donc, ils ne doivent prendre en compte que certains buts assignés à

917 Déclaration de l'Ambassadeur du Zimbabwé au Conseil de sécurité, voir Doc.S/ PV/ 3145, p.7.

918 DJIENA WEMBOU (Michel-Cyr), Le droit international dans un monde en mutation, op.cit., p.118.

$919 \quad$ Ibid, p.119. 
l'organisation par les États. Mais, dans les Chartes constitutives les États portent atteinte à l'efficacité des organisations internationales en étendant de façon considérable les pouvoirs des organes restreints. Le cas du Conseil économique et social de l'ONU nous paraît symptomatique.

Les rédacteurs de la Charte de l'ONU ont affaiblit l'autorité du Conseil économique et social par l'extension de ses pouvoirs. En effet, le Conseil économique et social s'intéresse à tous les secteurs de la coopération internationale, qui sétendent, aux termes de l'article 55, aux affaires économiques et sociales, aux problèmes de culture et déducation et aux questions relatives aux droits de l'homme et aux libertés fondamentales. ${ }^{920}$

Le réseau de relations dans lesquelles le Conseil économique et social est engagé est très complexe. ${ }^{921}$ Dans ce cadre, il est en relation étroite avec les institutions spécialisées, ${ }^{922}$ avec un grand nombre d'organisations internationales (ou nationales) non gouvernementales (O.N.G), s'intéressant à tel ou tel aspect des problèmes de sa compétence. ${ }^{923}$

Le Professeur VIRALLY souligne que " le champ ouvert aux activités du Conseil économique et social est de plus en plus démesuré, complexe et encombré d’un nombre croissant d'organismes internationaux les plus divers, de caractère public ou privé, dont le foisonnement appelle la coordination et, peut-être encore, des idées directrices et un mécanisme intégrant dans une vue d'ensemble leurs actions dispersées et les fondant dans une stratégie globale. " ${ }^{924}$ Cette situation le conduit à se poser une question fondamentale : « l'importance absolument primordiale du Conseil économique et social, au cœur du système des Nations Unies, qui lui donne un rôle décisif dans nombre de questions intéressant au premier chef la majorité des pays sous-développés, est-elle compatible avec son statut d'organe restreint? " ${ }^{925}$

Cette question est d’autant plus sérieuse que le Conseil est, en quelque sorte écrasé entre l'Assemblée générale, dont il dépend, et qui est un organe plénier, et les institutions spécialisées ou autres organisations qu'il coordonne. ${ }^{926}$ Le Conseil économique et social assume difficilement son rôle d'organe restreint du fait de la volonté des États fondateurs des Nations Unies qui lui ont définit des compétences trop importantes dans la Charte. Les conséquences de cette situation sur son fonctionnement sont énormes. Le poids et la diversité des questions dont le Conseil a à s'occuper, et qui rendent ses ordres du jour absolument démentiels. ${ }^{927}$ Beaucoup de temps est perdu par des tâches administratives ou réglementaires dépourvues de portée, telles que l'approbation des rapports provenant d'une multitude d’organe (Institutions spécialisées, Commissions économiques

\footnotetext{
920 VIRALLY (Michel), Lorganisation mondiale, op.cit., p.90.

921 Ibid, p.91.

922 Voir l'article 57 de la Charte des Nations Unies.

923 Voir l'article 71 de la Charte des Nations Unies.

924 VIRALLY (Michel), L'organisation mondiale, op.cit., p.91.

925 Ibid, p.93.

926 Ibid.

927 Ibid, p.92
} 
régionales, organismes divers), qui, en raison de leur nombre et de leur complexité, ne peuvent faire l'objet d'un examen sérieux. ${ }^{928}$

L'extension des pouvoirs des organes restreints dans les Chartes constitutives porte atteinte à son autorité. Mais en dehors de cela, il faut aussi mentionner les restrictions portées aux pouvoirs des organes restreints découlant du droit de véto institué dans les Chartes constitutives.

\section{B : LES RESTRICTIONS AUX POUVOIRS DES ORGANES RESTREINTS DECOULANT DU DROIT DE VETO}

En plus de son caractère hégémonique, ${ }^{929}$ le droit de véto constitue une limitation substantielle aux pouvoirs des organes restreints. Au sein d'un organe d'une organisation internationale, le vote exprime non pas la volonté individuelle des États mais la volonté de lorgane. Le Professeur René-Jean DUPUY souligne «qu'on distingue l'organisation internationale et la Conférence diplomatique classique, pièce maîtresse du système relationnel, en se fondant sur l'imputation des volontés des États à l’organisation ou aux États [...] Ainsi, lorsque dans une Conférence diplomatique un représentant exprime l'opinion de son État, il n’engage que celui-ci ; au contraire lorsque, dans une organisation internationale, le chiffre requis pour la réunion de la majorité, par exemple les deux tiers, a été atteint, le courant juridique, au lieu de revenir vers les États, est extraverti de ceux-ci vers l’organe ; le vote dégage alors la volonté de lorganisation ellemême, volonté cohésive et nouvelle, distincte de la volonté de chacun des États membres.» ${ }^{930}$ Le vote est donc l'expression de la volonté libre de l'organe d'une organisation internationale.

Mais, ce pouvoir d’exprimer librement la volonté de lorgane par le vote est entravé par le droit de véto. En instituant un droit de véto dans les organes restreints, les États, surtout les grandes puissances tendent véritablement à fusionner leur volonté individuelle à celle de l’organe. La volonté d’un seul État remet en cause la volonté générale exprimée par la majorité des États. La conséquence politique du privilège du véto, c'est qu'une grande puissance se servira du véto si, étant en minorité au Conseil, elle estime que ses intérêts sont menacés, soit directement, soit à travers les intérêts d'un autre État, par une décision de la majorité. ${ }^{931}$ L'illustration qui peut être donnée ici est le véto russe et chinois à un projet de résolution proposé par les pays occidentaux membres permanents du Conseil de sécurité le 4 octobre 2011 et le 4 février 2012 et le 19 août 2012 dans la crise syrienne. ${ }^{932}$ Par ces différents vétos, la Chine et le Russie empêchent le Conseil de sécurité de sortir de onze mois de silence sur la Syrie, pendant lesquels les affrontements armés ont fait au moins 6000 morts. Le désaccord entre les cinq grands constitue un obstacle au Conseil

928 VIRALLY (Michel), L’organisation mondiale, op.cit., p.91.

929 Voir le titre II de la première partie.

930 DUPUY (René-Jean), La communauté internationale entre le mythe et l'histoire, op.cit., p.49.

931 CHAUMONT (Charles), L'ONU, Collection Que sais-je ? n748, PUF, Paris, 1971, p.45.

932 Voir le site www.Le monde .fr avec Agence France Presse/ 05. 02.2012. 
de sécurité dans sa prise de décision. ${ }^{933}$ Mais dans le fond est-qu'une résolution autorisant le recours à la force dans la crise syrienne telle que le préconisent les pays occidentaux peut-elle être une solution idoine? Il est difficile de répondre par l'affirmative à cette interrrogation. Si dans les crises ivoiriennes et libyennes, le Conseil de sécurité a eu recours à l’usage de la force armée, à travers les résolutions 1975 et 1973, la crise syrienne a sa particularité. Chaque crise ayant sa spécificité, il est irréaliste de transposer les solutions des crises ivoiriennes et libyennes à la Syrie.

Le Professeur René DEGNI-SEGUI considère que le droit de véto prévu à article 27 de la Charte de l'ONU constitue une limite au pouvoir du Conseil de sécurité. ${ }^{934} \mathrm{Il}$ estime que le droit de véto est «une limitation considérable de la portée des fonctions et des pouvoirs du Conseil. Celui-ci, en effet, ne peut exercer ceux-là qu'avec l'accord unanime des cinq membres permanents, l'un quelconque d’entre eux, pouvant, par son droit de véto paralyser le Conseil et du même coup bloquer le système de sécurité collective. $\rangle^{935}$ Avec le droit de véto, le pouvoir réel du Conseil de sécurité est transféré aux membres permanents. L'existence de ce privilège provient d’une décision prise à la Conférence de Yalta de février 1945 entre les trois chefs d'État des principales puissances alliées : Monsieur W. Churchill, le Président Roosevelt et le maréchal Staline. ${ }^{936}$ Ainsi qu'il ressort d'une déclaration officielle faite le 7 juin 1945 par les États-Unis, le Royaume-Uni, l’URSS et la Chine, à la Conférence de San Francisco, «étant donné les responsabilités primordiales des membres permanents, on ne peut s'attendre, dans l'état actuel des conditions du monde, à ce qu'ils assument l'obligation d’agir dans un domaine aussi grave que le maintien de la paix et de la sécurité, en exécution d’une décision à laquelle ils n’ont pas donné leur adhésion. » ${ }^{937}$

A l'exception des membres permanents du Conseil eux-mêmes, de nombreux États, notamment ceux du tiers monde, ont critiqué violemment à l'Assemblée générale, le droit de véto accordé aux grandes puissances, et l'utilisation qu'ils en font. ${ }^{938}$ Le droit de véto constitue l'une des raisons principales qui justifient la réforme du Conseil de sécurité. A ce sujet, deux cas de

933 Les déclarations des différentes délégations des États suite au troisième véto russe et chinois après la $6810^{\mathrm{e}}$ séance du Conseil de sécurité témoignent du désaccord entre les membres permanents du Conseil de sécurité. Ainsi, Madame Susan RICE (États-Unis) a rappelé que « c'est la troisième fois en 10 mois que la Fédération de Russie et la Chine opposent leur droit de véto à un projet de résolution sur la Syrie. Ces vétos sont déplorables. [...] Malgré les déclarations paranoïques de certains, jamais ce projet n'aurait ouvert la voie à une action militaire ». Monsieur Li BAODONG (Chine) soutient que « ce projet a pour but de faire pression sur une partie seulement. Il risquait de générer un débordement dans d’autres pays de la région ». Quant à Monsieur Gérard ARAUD (France), il affirme qu' " il est désormais clair que le véto russe et chinois ne vise qu’à laisser plus de temps au régime syrien pour écraser l'opposition ». Enfin Monsieur Vitaly CHURKIN (Fédération de Russie) estime qu' « un vote n'aurait pas dû avoir lieu aujourd'hui, car les auteurs du projet de résolution savaient bien que ce texte n'avait aucune chance dêtre adopté. Nous ne pouvons pas accepter un document qui ouvre la voie à des sanctions et puis à une intervention militaire ». Voir Conseil de sécurité/ 10714/ Département de l'information, service des inforamations et des accéditations, New York, 19/07/2012. DEGNI-SEGUI (René), « Commentaire de l’article 24 paragraphe 1 et 2 de la Charte de l'ONU », op.cit., p.464.

935 Ibid.

936 CHAUMONT (Charles), L’ONU, Collection Que sais-je ? n748, op.cit., p.44.

937 Ibid

938 DJIENA WEMBOU (Michel-Cyr), Le droit international dans un monde en mutation, op.cit., p.94. 
figure se présentent à l'ensemble des États. Dans une première hypothèse, les États s'accordent sur la suppression du droit de véto. Mais, elle semble manifestement irréaliste dans la mesure où le droit de véto est le reflet des rapports de force entre les grandes puissances et les petits États. En revanche, le droit de véto pourrait être élargi à de nouvelles puissances tels que l'Inde, le Japon, l’Afrique du Sud et le Brésil.

Les pouvoirs du Conseil d'administration du FMI sont aussi fortement limités par le droit de véto dont dispose les États Unis. Les pouvoirs du Conseil d’administration du FMI sont détenus par cet État en raison de ses 17\% aux parts du capital de cette organisation. Ces 17\% au capital du FMI permettent aux États-Unis de bloquer les votes les plus importants où une majorité de $85 \%$ est nécessaire. ${ }^{939}$

Les États qui ont créé les organisations internationales ont imposé des restrictions aux pouvoirs des organes restreints dans les Chartes constitutives. Cependant, en marge de ces limitations constitutionnelles, il y a aussi celles qui sont souhaitables pour les États. Il s'agit du contrôle de constitutionnalité des actes du Conseil de sécurité.

\section{PARAGRAPHE II : LES LIMITATIONS SOUHAITABLES POUR LES ÉTATS: LE CONTRÔLE DE CONSTITUTIONNALITE DES ACTES DU CONSEIL DE SECURITE DES NATIONS UNIES}

Dans l'ordre interne des États, le contrôle de constitutionnalité est un mécanisme qui permet à un juge de vérifier la conformité d'un acte juridique à la Constitution. Ce contrôle permet d'assurer le respect de la Constitution qui est la norme supérieure au sein d’un État.

Dans l'ordre international, étant donné que les pouvoirs attribués au Conseil de sécurité sont exorbitants et discrétionnaires, la question du contrôle de constitutionnalité se pose avec acuité. Ce contrôle est souhaitable pour les Etats dans la mesure où il consistera à vérifier que les actes du Conseil ne violent pas la Charte et le droit international. ${ }^{940}$ Dans cette thèse, nous montrerons les enjeux (A) et la position de la CIJ sur la question (B).

939 Voir le site www.lefigaro.fr/économie/ 2009

940 Ce contrôle relève du souhaitable pour les États dans la mesure où aucun contrôle de constitutionnalité n’a été prévu dans le système de la Charte des Nations Unies. Or depuis 1990, le Conseil de sécurité a mis en œuvre ses pouvoirs de façon démesurée. Cette nouvelle tournure prise par le Conseil qui appelle nécessairement une limitation de ses pouvoirs constitue l'un des points les plus abordés par la doctrine ces dernières années. Ainsi, théodore Christakis montre clairement cette évolution du Conseil de sécurité (Voir à ce sujet, Théodore CHRISTAKIS, " Prométhée déchaînée ", Ares, n55, mai, 2005) : -Le Conseil de sécurité a procédé en 1991 à la démarcation d'une frontière litigieuse entre deux États souverains en tranchant un différend frontalier qui opposait l'Irak et le Koweït.

-Le Conseil a imposé la même année un régime de responsabilité et de réparation à l'Irak en mettant en place les organes et la procédure nécessaires à cet égard.

- Une année plus tard le Conseil a demandé à un État d’extrader ses ressortissants en écartant l'application d'une Convention internationale...etc 


\section{A : LES ENJEUX DU CONTRÔLE DE CONSTITUTIONNALITE}

Parler des enjeux du contrôle de constitutionnalité des actes du Conseil de sécurité revient à montrer l'intérêt de cette question en droit international public. Cette précision faite, il est important de souligner que l'importance que les États accordent à cette question est significative et particulière en ce sens qu'elle est intimement liée à la démocratisation de cet organe restreint. Si le Conseil semble non démocratique, ce n'est pas seulement à cause du nombre réduit de membres impliqués dans le processus de prise de décision, mais aussi en raison de l'absence de contrepoids ou de contrôle par un autre organe de l'organisation. ${ }^{941}$

Contrairement au Conseil de sécurité, les autres organes restreints visés dans cette étude sont dans une situation de dépendance juridique vis-à-vis des organes pléniers. ${ }^{942} \mathrm{Du}$ fait de cette dépendance juridique les actes adoptés par ces organes sont directement censurés par les États à travers les organes pléniers. Dans ce cas, il ne s'agit pas d'un contrôle juridictionnel mais d'un contrôle politique. En revanche dans le cas du Conseil de sécurité, l'Assemblée générale étant juridiquement incompétente pour contrôler la conformité à la Charte et au droit international, des actes adoptés par le Conseil de sécurité, la question prend une dimension particulière. ${ }^{943}$ Donc, un contrôle par un organe juridictionnel s'impose. Ce contrôle est souhaitable pour les États pour des raisons qui se situent tant au plan juridique que politique.

Au plan juridique, aux termes de l'article 39 de la Charte, le Conseil de sécurité jouit d'un pouvoir discrétionnaire pour constater l'existence d'une menace à la paix, d'un acte agression, et d'une rupture de la paix. ${ }^{944}$ Au regard de cette disposition, le Conseil bénéficie d’un pouvoir illimité dans la mesure où il doit non seulement établir ce qui sest effectivement passé, apprécier et qualifier les faits à la manière d'un juge. ${ }^{945} \mathrm{Or}$, en droit toute opération de qualification implique toujours le pouvoir prétorien de celui qui qualifie. Peut-on considérer que le Conseil de sécurité est libre de qualifier n'importe quelle situation de menace contre la paix, de rupture de la paix ou d'un acte d’agression ?946 Autrement dit, les pouvoirs énoncés par le Chapitre VII peuvent-ils être utilisés à la pleine discrétion du Conseil ?947

Pour le juge Mohamed BEDJAOUI «le États ne peuvent échapper à la sanction de leurs actes internationalement illicites. Il paraît alors de moins en moins cohérent aujourd'hui que

941 FLEURENCE (Olivier), La réforme du Conseil de sécurité, létat du débat depuis la fin de la guerre froide, op. cit., p.250.

942 Voir à ce sujet la section suivante qui porte sur la limitation des pouvoirs des organes restreints par les organes pléniers.

943 L'Assemblée générale et le Conseil de sécurité sont des organes principaux placés dans une situation d'autonomie juridique dans la Charte des Nations Unies.

944 Voir l'article 39 de la Charte des Nations Unies.

945 COHEN JONATHAN (Gérard), "Commentaire de l’article 39 de la Charte des Nations Unies », in COT (JeanPierre), PELLET (Alain), La Charte des Nations Unies, commentaire article par article, $2^{\mathrm{e}}$ édition, op.cit., p.651.

946 GAJA (Georgio), Réflexion sur le rôle du Conseil de sécurité dans le nouvel ordre mondial », op.cit., p.298.

947 Ibid. 
l'organisation universelle qu'ils ont eux- même créée puisse quant à elle demeurer immune de tout contrôle de légalité des actes de ses organes politiques et de toute sanction de leur dépassement.» ${ }^{948}$ Ainsi, au plan juridique, les organes des organisations internationales en général doivent être soumis au respect de leurs Chartes constitutives et du droit international. Cette idée est partagée par le Professeur Benedetto CONFORTI qui estime que " les organes des Nations Unies sont tenus de se conformer à la Charte [...] mais aussi aux principes du droit international général ou commun.» ${ }^{949}$ Les pouvoirs du Conseil de sécurité reposent sur l'habilitation qui lui a été conféré par la Charte des Nations et, à travers elle, par les États. ${ }^{950}$

Au plan politique, les enjeux d'un contrôle de constitutionnalité des actes du Conseil de sécurité se justifient aussi par la quête d'un nouvel ordre politique international marqué par la démocratisation des relations internationales. La démocratie est devenue tant au sein des organisations internationales qu'en dehors de celles-ci le nouveau mythe mobilisateur des temps nouveaux. ${ }^{951}$ Ainsi, s'interroge le juge BEDJAOUI : «comment concevoir une réelle démocratisation des Nations Unies sans poser entres autres le problème fondamental du contrôle de la légalité des actes des organes politiques, et singulièrement de la pièce maîtresse que constitue le Conseil de sécurité ?» ${ }^{952}$ Ainsi, de nombreux Etats soulignent la nécessité pour le Conseil de rester dans le cadre de la Charte. La Colombie évoque la possibilité d'un contrôle juridictionnel sur les actions du Conseil de sécurité. ${ }^{553}$ Dans sa déclaration, le représentant de l'Egypte appuie également cette idée en insistant sur le fait que les actes du Conseil de sécurité « could, if the need arose, be subject to judicial review by the international court of justice to ensure that the rule of law and justice is paramount $»{ }^{954}$ Le délégué de l'Algérie aborde la question en faisant relever l'activation dudit contrôle de la compétence de l'Assemblée générale. ${ }^{955}$

Le nouvel ordre mondial et le Conseil de sécurité qui devrait être son bras séculier, ne peuvent gagner en crédibilité, en autorité et en efficacité que si la conviction est bien acquise qu'ils agissent non pas en institutions au dessus de la Charte et du droit international, mais bien en qualité de serviteurs de ceux-ci. ${ }^{956}$

La revendication d'un contrôle de constitutionnalité des actes du Conseil de sécurité est motivée par le respect du droit international et la Charte constitutive des organisations Shabtai ROSENNE, Boston/London, Martinus Nijhoff publishers, 1999, p.75.

949 CONFORTI (Benedetto), « Rôle de l'accord dans le système des Nations Unies », RCADI, Tome 142,1974-II, p.209.

950 DE BRICHAMBAUT (Marc), DOBELLE (Jean-François), D’HAUSSY (Marie-Reine), Leçons de droit international public,Presses de sciences Po et Dalloz,Paris, 2OO2,, p.273.

951 BEDJAOUI (Mohamed), « Du contrôle de la légalité des actes du contrôle de sécurité », op.cit., p.74.

$952 \quad$ Ibid, p.75.

953 A/48/PV.62,p.20.

$954 \mathrm{~A} / 48 /$ pv.61, p.17.

955 A/48/ PV.62, p.17.

$956 \quad$ Ibid, p.76. 
internationales; mais aussi par la volonté de démocratisation des relations internationales. Mais, au-delà de ce vœux, quelle est la position de la CIJ sur la question ?

\section{B : LA POSITION DE LA CIJ}

Aux termes de l'article 92 alinéa 1 de la Charte des Nations Unies, «la CIJ constitue l'organe judiciaire principale des Nations Unies.» ${ }^{957}$ La CIJ est donc un organe juridictionnel de l'ONU. Elle correspond bien à la définition que donne le Professeur GUZMAN: « one could limit the definition of international tribunals to bodies that have explicitly been granted authority by the parties to rule on legality of dispute conduct.» ${ }^{958}$

Elle est placée au centre de la question du contrôle de constitutionnalité des organes restreints. Sa compétence en la matière découle de l’article 96 de la Charte de l'ONU et 34 alinéa 1 de son Statut. Il ressort de l'article 96 de la Charte de l'ONU que «l'Assemblée générale ou le Conseil de sécurité peut demander à la CIJ un avis consultatif sur toute question juridique. Tous les autres organes de l'organisation et institutions spécialisées qui peuvent, à un moment quelconque, recevoir de l'Assemblée générale une autorisation à cet effet ont également le droit de demander à la Cour des avis consultatifs sur des questions juridiques qui se poseraient dans le cadre de leur activité.» ${ }^{959}$ Cette disposition permet à la CIJ de donner son opinion sur des questions juridiques émanant de l'Assemblée générale ou du Conseil de sécurité, des organes de l'organisation et des institutions spécialisées qui ont reçu une autorisation de l'Assemblée générale. Il s’agit là de la compétence consultative de la CIJ.

En revanche, la compétence contentieuse de la CIJ est prévue par l'article 34 alinéa 1 de son Statut. Selon cette disposition «seuls les États ont qualité pour se présenter devant la Cour...»"${ }^{960}$ La fonction contentieuse qui est la plus importante de la CIJ ou tout au moins la plus «évidente» de la CIJ est de régler les litiges entre les États. ${ }^{961}$

Qu'il s'agisse de sa fonction consultative ou de sa fonction contentieuse, la CIJ a été saisie à plusieurs reprises sur des affaires faisant ressortir la question du contrôle de la légalité des actes du Conseil de sécurité. Aussi, dans son avis consultatif du 21 juin 1971 relatif aux conséquences de la présence continue de l'Afrique du Sud en Namibie, cette question s'est présentée à la Cour. Rappelons brièvement les faits à l'origine de cet avis consultatif. En effet, les Nations Unies accentuent leurs efforts dans la question du Sud-Ouest africain, désormais dénommé Namibie. Le Conseil de sécurité adopte une résolution 276 (1970) sur la question réaffirmant que «le mandat sur le Sud-

957 Voir l'article 92 de la Charte des Nations Unies.

958 GUZMAN (Andrew T.), «International tribunal : a rational choice analysis », UC Berkeley public law and legal theory working paper, op.cit., p.184.

959 Voir l'article 96 de la Charte des Nations Unies.

960 Voir l'article 34 alinéa 1 du Statut de la CIJ.

961 LE JEUNE (Pierrick), Introduction au droit des relations internationales, op.cit., p.131. 
Ouest africain est terminé» et que «la présence continue des autorités Sud-africaines en Namibie est illégale» et demande à tous les États de s'abstenir de toutes relations avec le gouvernement Sudafricain. Le Conseil de sécurité adopte alors une résolution du 29 juillet 1970 demandant un avis à la Cour sur la question suivante :

«Quelles sont les conséquences juridiques de la présence continue de l’Afrique du Sud en Namibie, nonobstant la résolution 276 du Conseil de sécurité ?»»62

L'intérêt de cet avis relativement au problème du contrôle de la Constitutionnalité des actes du Conseil de sécurité se situe au niveau de l'exception préliminaire soulevée par l'Afrique du Sud sur la conformité à la Charte de la résolution 276. ${ }^{963}$ Dans cette exception préliminaire, l'Afrique du Sud conteste la constitutionnalité de la résolution 276 au motif que deux membres permanents du Conseil de sécurité (la Russie et la France) sétaient abstenus et l'Afrique du Sud avait été absente aux débats.

Dans son avis, la CIJ soutient que «toute résolution émanant d'un organe des Nations Unies régulièrement constitué, prise conformément à son règlement et adoptée par son président, doit être présumée valable.» ${ }^{964} \mathrm{La}$ CIJ pose donc le principe de la présomption de validité des résolutions du Conseil de sécurité. Mais, cette thèse est contestable. La présomption de validité ne trouve aucun appui dans les dispositions ou les principes de la Charte. ${ }^{965}$ Aussi, la CIJ aurait pu contrôler la conformité à la Charte de la résolution 276 avant de statuer au fond. Le juge André GROS, dans son opinion dissidente montre l'incohérence de cette théorie en ces termes : «comment, en effet, un juge peut-il déduire une obligation d'une situation quelconque sans avoir d'abord éclairci la question de la légalité des origines de cette situation.» ${ }^{966}$

Comme on peut le constater, la CIJ dans sa fonction consultative ne sest pas prononcée sur la constitutionnalité de la résolution 276 du Conseil de sécurité. Sûrement, sa compétence contentieuse lui permettra de préciser sa jurisprudence. Cette question du contrôle est apparue pour la première fois à titre contentieux devant la CIJ dans le cadre de l'affaire «des questions d'interprétation et d'application de la Convention de Montréal de 1971 résultant de l'incident aérien de Lockerbie, l'opportunité d’un tel contrôle sétait présentée à la Cour. ${ }^{967}$ Dans cette affaire, le Conseil de sécurité avait adopté la résolution 731 enjoignant la Libye, sous peine de sanctions, d'extrader les auteurs présumés d’avoir commis l’attentat contre le Boeing de la Pan Am qui explosa

962 La jurisprudence de la CIJ de 1946 à 1971, Notes et études documentaires, Paris, la documentation française, 15 mai 1972, p.30.

963 Les exceptions préliminaires sont des moyens invoqués au cours de la première phase de l'instance et tendant à obtenir que le tribunal tranche une question préalable avant d'obtenir qu'il ne soit passé à l'examen du fond.

964 La jurisprudence de la CIJ de 1946 à 1976, p.22.

965 CONFORTI (Benedetto), « Rôle de l'accord dans le système des Nations Unies », op.cit., p.232.

966 Recueil des arrêts de la CIJ, 1971, p.332.

967 DE BRICHAMBAUT (Marc Perrin), DOBELLE (Jean-François), D’HAUSSY (Marie-Reine), Leçons de droit international public, op.cit., p.273. 
au dessus de Lockerbie (Ecosse) le 21 décembre 1988. ${ }^{968}$ La Libye saisit la CIJ pour lui demander de prescrire des mesures conservatoires de ses droits. ${ }^{969}$ Le gouvernement libyen demanda à la Cour d'indiquer des mesures conservatoires interdisant la Grande-Bretagne et les États-Unis d'engager contre la Libye une action visant à la contraindre de procéder à cette extradition.

Mais le 31 mars 1992, le Conseil de sécurité, devant le refus persistant de la Libye, décida de faire application du Chapitre VII de la Charte, estimant que le « défaut de la part du gouvernement libyen de démontrer, par des actes concrets sa renonciation au terrorisme...constituait une menace contre la paix et la sécurité internationale.» La résolution 748 édicta à l'égard de la Libye un embargo sur les livraisons d'armes et les relations aériennes, ainsi qu'une réduction de l'importance des missions diplomatiques. ${ }^{970}$

Le 14 avril 1992, la Cour prit une ordonnance sur les mesures conservatoires demandées par la Libye. Par 11 voix contre 5, la Cour a refusé de prescrire des mesures conservatoires eu égard «aux circonstances de l'espèce». Elle a estimé que l'indication de telles mesures porterait atteinte aux droits que les États-Unis et la Grande-Bretagne peuvent tirer de la résolution 748. ${ }^{971}$

La résolution 748 a été contestée au motif qu’elle fut adoptée alors que la Libye avait déjà introduit une requête devant la $\mathrm{CIJ}$ pour faire reconnaître son droit à ne pas extrader ses nationaux, droit quelle prétend tenir de la Convention de Montréal de 1971 pour la répression d’actes illicites portant atteinte à la sécurité de l'aviation civile. ${ }^{972}$ En outre dans son contenu, cette résolution s'appuie sur une déclaration commune des États-Unis et de la Grande-Bretagne qui «exigent la livraison des personnes accusées, la divulgation par la Libye de tous les renseignements nécessaires et le versement d'indemnités.» ${ }^{973}$ Manifestement, la formulation très discutée et pour le moins maladroite du texte américano-britannique qui demande la livraison des deux «accusés» et le versement immédiat d'indemnités mérité un éclaircissement. ${ }^{974}$ La présomption d'innocence disparaît et la qualification «d'accusé» est donnée non pas par un jury populaire mais par les États. Le versement d'indemnités exigé semble faire fi de toute la procédure de la responsabilité internationale. ${ }^{975}$ En d'autres termes, c'est réclamer la réparation avant d’avoir établi la culpabilité des suspects qui -si elle est reconnue- apporte la preuve de la culpabilité de la Libye comme

968 KERBRAT (Yann), La référence au Chapitre VII de la Charte des Nations Unies dans les résolutions à caractère humanitaire du Conseil de sécurité, LGDJ, Paris, 1995, p.29.

969 La mesure conservatoire est une mesure provisoire destinée à assurer qu'en attendant le règlement de droits contestés, ceux-ci soient conservés.

970 RUZIE (David), Droit international public, Mémentos Dalloz, $18^{e}$ édition, Paris, 2006, p.244.

971 Ibid.

972 MAZERON (Florent), «Le contrôle de légalité des décisions du Conseil de sécurité- Un bilan après les ordonnances Lockerbie et l'arrêt Tadic », Revue québécoise de droit international, 1997, p.116.

973 SOREL (Jean-Marc), « Les ordonnances de la CIJ du 14 avril 1992 dans l'affaire relative à des questions d'interprétation et d’application de la Convention de Montréal de 1971 résultant de l'incident aérien de Lockerbie (Libye contre Royaume Uni et Libye contre États- Unis) », RGDIP, 1993, p.721.

Ibid.

975 Ibid. 
un État terroriste. ${ }^{976}$ Enfin, il faut souligner qu'en exigeant à la Libye la divulgation de tous les renseignements nécessaires à la manifestation de la vérité, nous assistons à un exemple unique d'imposition d'une coopération judiciaire forcée.

Au regard de ce qui précède, la CIJ a eu la possibilité de contrôler la conformité de la résolution 748 à la Charte des Nations Unies et au droit international. ${ }^{977}$ Bien au contraire, la CIJ a esquivé ce contrôle au motif « qu'au stade de l'indication de mesures provisoires, elle n’avait pas à se prononcer définitivement sur l'effet juridique de la résolution $748 . »^{978}$ La question de la conformité des résolutions à la Charte et au droit international général n’a pas été évoquée, car elle a été considérée comme un argument relevant du fond. ${ }^{979}$

Cette position de la Cour est amplement justifiée dans la mesure où «le système des Nations Unies issu de la Conférence de San Francisco ne prévoit ni contrôle judiciaire de la légalité, ni système unifié d'interprétation de la Charte...» ${ }^{980} \mathrm{~A}$ sa création, les États n’ont pas eu la volonté d'en faire une Cour constitutionnelle. Aucune disposition de la Charte de l'ONU et du Statut de la CIJ n’autorise le contrôle de constitutionnalité des actes du Conseil de sécurité.

Les fondateurs des Nations Unies auraient souhaité que s'instaure un mécanisme juridictionnel de contrôle de constitutionnalité des actes juridiques du Conseil de sécurité. Ce souhait est vivement exprimé par la majorité des petits États en raison des pouvoirs exorbitants de cet organe dans le domaine du maintien de la paix et de la sécurité internationales.

Mais en marge des limitations imposées ou voulues par les États, il y a celles qui émanent des organes pléniers. Du fait de leur représentativité, dans la majorité des organisations internationales les organes pléniers limitent les compétences des organes restreints.

\footnotetext{
$976 \quad$ Ibid.

977 MAZERON (Florent), «Le contrôle de la légalité des décisions du Conseil de sécurité, un bilan après les ordonnances Lockerbie et l'arrêt Tadic », op.cit., p.118.

978 GAJA (Georgio), « Réflexions sur le rôle du Conseil de sécurité dans le nouvel ordre mondial », RGDIP, 1993, p.317.

979 DE BRICHAMBAUT (Marc Perrin), DOBELLE (Jean-François), D’HAUSSY (Marie-Reine), Leçons de droit international public, op.cit., p.274.

980 MAZERON (Florent), «Le contrôle de la légalité des décisions du Conseil de sécurité, un bilan après les ordonnances Lockerbie et l'arrêt Tadic », op.cit., p.124.
} 


\section{CHAPITRE II : LES LIMITATIONS DES POUVOIRS DES ORGANES RESTREINTS PAR}

\section{LES ORGANES PLENIERS}

Les organes pléniers, expression achevée de la démocratie directe du fait de la représentation de tous les États membres limitent les compétences des organes restreints. Cette limitation se réalise par la dépendance des organes restreints vis-à-vis des organes pléniers (Section I) et le contrôle des organes restreints par les organes pléniers (Section II). 
Etant constitué d'un nombre limité de membres, il est tout à a fait normal que les organes restreints dépendent des organes pléniers. Cette dépendance se traduit tant par la désignation des membres (Paragraphe I) que par la réduction des compétences des organes restreints par les organes pléniers (Paragraphe II).

PARAGRAPHE I : LA DESIGNATION DES MEMBRES DES ORGANES RESTREINTS PAR LES ORGANES PLENIERS

Généralement, dans les organisations internationales les membres des organes restreints sont désignés par l'ensemble des États membres siégeant dans les organes pléniers. Cette désignation se fait dans la majorité des cas par la voie des élections (A). Mais, il est important de souligner la forte politisation qui entoure ces élections (B).

\section{A : L'ELECTION DES MEMBRES DES ORGANES RESTREINTS PAR LES ORGANES PLENIERS}

Au regard des différentes Chartes constitutives des organisations internationales, un constat s'impose. La composition des organes restreints dépend généralement des organes pléniers. Dans la plupart des organisations internationales, les membres des organes restreints sont élus ou nommés par les organes pléniers.

Larticle 23 de la Charte des Nations Unies dispose que «... dix autres membres de lorganisation sont élus, à titre de membres non permanents du Conseil de sécurité, par l'Assemblée générale... ${ }^{981}$

Quant à l'article 61, il prévoit que «le Conseil économique et social se compose de cinquante-quatre membres de l'organisation des Nations Unies, élus par l’Assemblée générale.... ${ }^{982}$

En ce qui concerne la Commission de l'UA, aux termes de l'article 9 alinéa (i) de l'Acte constitutif, il incombe à la Conférence des Chefs d'État et de gouvernement de «nommer le Président, le ou les vice-présidents et les commissaires de la commission... ${ }^{983}$

981 Voir l'article 23 de la Charte des Nations Unies.

982 Voir l'article 61 de la Charte des Nations Unies.

983 Voir l'article 9 de l'Acte constitutif de l'UA. 
Quant au Conseil de Paix et de sécurité de l'UA, les membres de cet organe restreint sont élus par la Conférence des Chefs d'État et de gouvernement ${ }^{984}$ conformément à l'article 5 alinéa 2 du protocole relatif à la création de cet organe.

Aux termes de l'article XII section 3 du Statut du FMI, parmi les vingt administrateurs composant le Conseil d’administration quinze sont élus par les États membres. ${ }^{985}$

Enfin, il ressort de l'article 161 de la Convention de MONTEGOBAY que «le Conseil de l'Autorité des fonds marins se compose de trente-six membres élus par l’Assemblée.» ${ }^{986}$

La désignation des membres des organes restreints des organisations internationales traduit fondamentalement une volonté politique des membres des organes pléniers. En réalité, soutient Monsieur BOUTROS-GHALI, «si l’on adopte la démocratie comme principe de base, il faut donner à l’organe général les pouvoirs qui doivent lui appartenir dans un système démocratique, puisqu'il constitue, dans l'organisation internationale la seule institution pleinement représentative.» ${ }^{987}$ Logiquement, cette élection des membres des organes restreints est révélatrice de la primauté politique et fonctionnelle des organes pléniers. Dans les organisations internationales, les organes pléniers expriment clairement la volonté générale et sont plus représentatifs de l'ensemble des États membres. Donc, le pouvoir de désignation des membres des organes restreints devrait relever de leur compétence.

Cette règle semble être bien perçue dans les Chartes constitutives. Mais, une discrimination est établie en faveur des membres non élus : ce sont les membres permanents. Ils jouissent d'un siège inamovible en raison de leur puissance économique et politique.

Le droit de désignation des membres des organes restreints vise à atténuer les effets de l'inégalité des États dans les organisations internationales. Ne siégeant pas tous dans les organes restreints, les membres des organes pléniers expriment par ce pouvoir leur suprématie politique. Mais, ce pouvoir des membres des organes pléniers est mis en mal par la politisation qui entoure lélection des membres des organes restreints.

\section{B : LA POLITISATION DE L'ELECTION DES MEMBRES DES ORGANES RESTREINTS}

Lélection des membres des organes restreints est fortement influencée par des considérations politiques. En réalité, cette élection est une question essentiellement politique à laquelle se mêle le jeu d'influence, les rapports de force et d'intérêts entre les États membres. Les

984 DUJARDIN (Stéphanie), «l'UA, objectifs et moyens de gestion des crises politiques et des conflits armés », in l'UA et les acteurs sociaux dans la gestion des crises et des conflits armés, actes du Colloque du 17 novembre 2005, Sénat, Paris, l'harmattan, 2006, p.63.

985 Voir l'article XII, section 3 du Statut du FMI.

986 Voir l'article 161 de la Convention de MontegoBay.

987 BOUTROS-GHALI (Boutros), «Le principe dégalité des États et les organisations internationales », op.cit., p.36. 
organes restreints jouant un rôle essentiel dans le fonctionnement des organisations internationales, loccupation des différents sièges présente un intérêt politique majeur pour les États membres.

La Commission de l'UA n’a pas échappé à ces contradictions politiques lors de lélection de son premier Président. ${ }^{988}$ Deux personnalités issues de l'Afrique de l'ouest, en l'occurrence Monsieur Essy AMARA, ancien Ministre des affaires étrangères de la Côte d'Ivoire et Monsieur Alpha Oumar KONARE, ancien Chef d'État du Mali ont fait acte de candidature pour le poste de Président de la Commission. Ces deux candidatures ouest africaines ont alimenté des rivalités politiques au sein de la sous région. En effet, la raison principale des débats suscités par ces deux candidatures était liée au fait qu'il était anormal que deux personnalités de la même zone géographique postulent pour le poste de Président de la Commission de l'UA.

Pour résoudre ce dilemme, la solution idoine qui se présentait était le retrait d’une des deux candidatures. Dans cette perspective, deux positions se sont élevées ; l'une soutenue par les partisans de Monsieur KONARE qui estimaient que pour le prestige de la Commission, il était souhaitable qu'une personnalité de haut rang devienne le Président. ${ }^{999}$ Ce point de vue, bien qu’ayant le mérite de revaloriser le statut du Président de la Commission, fut battu en brèche par les partisans de Monsieur Essy AMARA. Pour l'ex-Président de la Côte d'Ivoire Laurent GBAGBO, «la candidature de Monsieur Konaré pourrait créer un précédent dangereux pour la Commission. En effet, élire Monsieur Konaré conduirait à transformer le poste de Président de la Commission en un poste de retraite pour les anciens Chefs d'État africains.» ${ }^{990}$ A ce propos il souligne : «Amara ESSY est l'un des diplomates de carrière les plus connus en Afrique. A Lusaka, ils étaient tous entre eux. Ce qui me fait dire que les diplomates forment une nouvelle ethnie, qu'ils sont entre eux, et quand il y a des candidatures, c'est entre eux. Aujourd'hui, nous sommes dans un cas de figure qui est différent, et c'est ce cas de figure que j’ai commencé à discuter avec les Chefs d'État, et que je discuterai avec eux jusquà ce que le moment des élections arrivent. Il faut comprendre, c'est un débat de fond sur la notion de Chef d'État en Afrique : je ne souhaite pas qu'un ancien Chef d'État soit candidat à un poste subalterne. Est-ce que, oui ou non, nous qui donnons les ordres au Président de la Commission, comme exécutant, demain il faut que nous nous retrouvions à ce poste comme exécutant des ordres que nous avons donné hier? Tel est le débat. Et il est de fond. Quelle

988 Cette élection a eu lieu lors de la deuxième Conférence des Chefs d'État et de gouvernement de l'UA du 4 au 12 juillet 2001 à Maputo, la capitale mozambicaine.

989 Cette position se justifiait dans la mesure où il fallait éviter que la Commission ne tombe dans une léthargie à l'instar du Secrétariat général de la défunte OUA. Un ancien Chef d'État au poste de Président de la Commission pourrait donner une bonne image de cet organe devant la Conférence des Chefs d'État et de gouvernement.

990 Sur ce fondement, la candidature de Monsieur Essy AMARA présentait une garantie d’efficacité au poste de Président de la Commission de l'UA. En sa qualité de diplomate expérimenté, il aurait pu valablement apporter son expérience à la nouvelle organisation africaine en gestation. 
est aujourd'hui la manière dont nous projetons les Chefs d'État en Afrique, pendant sa fonction et après ? C'est une question de vision et il faut que l'Afrique choisisse.. ${ }^{911}$

Un compromis politique a été trouvé à ce dilemme. Monsieur Essy AMARA a dû retirer sa candidature au profit de celle du Président Konaré, qui par la suite fut élu Président de la Commission de l'UA.

Un autre exemple mérite amplement dêtre cité encore pour montrer le degré de politisation considérable qui entoure lélection des membres des organes restreints. Il s'agit ici de l'élection du dixième membre non permanent du Conseil de sécurité lors de la $34^{\text {ème }}$ session de l'Assemblée générale en 1979. A cette élection, Cuba et la Colombie étaient les deux États latinoaméricains candidats au siège de membre non permanent. Malgré un léger avantage pour Cuba, aucun des deux États n’a pu obtenir la majorité requise. Après 154 tours de scrutin infructueux les deux États se retirèrent au profit du Mexique. ${ }^{992}$ Au regard de ces faits, on pourrait objectivement se poser la question de savoir pourquoi l'Assemblée générale de l'ONU, lors de sa trente-quatrième session, a-t-elle éprouvé autant de difficultés à fixer son choix sur l’un ou l'autre candidat?

Selon le Professeur Birame NDIAYE, «il est permis de penser que les interminables balancements de l'Assemblée générale avaient pour causes l'absence de consensus au sein du groupe latino-américain autour d'une candidature, $[\ldots]$, ayant pour soubassement la bipolarisation de la compétition Cuba-Colombie.» ${ }^{993}$

L'absence d'un consensus au sein du groupe latino américain se justifiait par le fait que dès le départ Cuba et la Colombie sont apparus comme étant respectivement les candidats des ÉtatsUnis et de l'URSS. La présence de La Havane au Conseil de sécurité ayant été jugée inacceptable par et pour Washington. ${ }^{94}$ Cette situation de blocage était du reste confirmée par les candidats qui faisaient valoir qu'il ne leur est pas possible de transiger pour sortir de cette impasse parce qu'ils représentent des blocs d’opinion qui englobent des secteurs de l'Assemblée générale, et par conséquent l'impasse ne concerne pas les deux États à titre individuel, mais également tous les membres de l’Assemblée. L'inaptitude à élire le $15^{\mathrm{e}}$ membre du Conseil de sécurité est donc une responsabilité collective. ${ }^{995}$ C’est finalement le Mexique, plus neutre, qui fut élu.

Il faut également rappeler qu’en 2006 un problème similaire s'est posé cette fois entre le Guatemala, un candidat réputé « mondialiste » contre un candidat réputé «altermondialiste»,

\footnotetext{
991 Voir le quotidien gouvernemental ivoirien «Fraternité Matin », dans sa parution du mercredi 21 mars 2003, à la page 24 .

992 N’DIAYE (Birame), "L'incapacité temporaire de l’Assemblée générale de l'ONU à élire le dixième membre non permanent du Conseil de sécurité », AFDI, 1980, p. 483.

993 Ibid.

994 N’DIAYE (Birame), «L'incapacité temporaire du Conseil de sécurité à élire le dixième membre non permanent du Conseil de sécurité », op.cit., p.483. Ibid, p.486.
} 
le Venezuela. ${ }^{996}$ Après 47 tours de scrutin, au 2 novembre 2006, ni le Guatemala, ni le Venezuela n’avaient réussi à obtenir les 128 voix nécessaires (deux tiers des votants à l'Assemblée générale qui compte 192 États membres). C'est finalement au profit du Panama, candidat de consensus, que les deux se retireront. Le 07 novembre 2006, le Panama est élu membre non permanent au Conseil de sécurité pour deux ans, représentant le groupe des pays d’Amérique latine et des Caraïbes.

La politisation des critères de sélection des membres non permanents des organes restreints réduit le pouvoir des membres des organes pléniers.Comme l’Assemblée plénière, lorgane restreint est le plus souvent le miroir passif des tensions traversant le monde qu'un instrument dynamique pour l'action. ${ }^{997}$ Les innombrables clivages divisant les États dans lorgane plénier pénètrent l'organe restreint et pèsent sur ses membres. ${ }^{998}$

Les limitations des pouvoirs des organes restreints peuvent résulter de la désignation par l’organe plénier des membres des organes restreints. Mais en dehors de cette hypothèse, lorgane plénier peut aussi limiter les compétences des organes restreints en leur attribuant des fonctions réduites.

\section{PARAGRAPHE II : LA REDUCTION DES COMPETENCES DES ORGANES RESTREINTS PAR LES ORGANES PLENIERS}

Les organes pléniers limitent les organes restreints en leur conférant des compétences purement administratives (A) ou encore essentiellement spécialisées (B).

\section{A : DES COMPETENCES PUREMENT ADMINISTRATIVES}

Les compétences administratives sont relatives au fonctionnement régulier et quotidien de l'organisation. En réalité, dans les organisations internationales ces compétences se résument à l’exécution des décisions des organes pléniers.

Les fondateurs des organisations internationales, par le truchement de la Charte constitutive, prennent généralement le soin d’empêcher une quelconque égalité fonctionnelle entre les organes pléniers et les organes restreints. A cet effet, dans la plupart des organisations internationales les organes pléniers, en leur qualité d’organes suprêmes confinent les organes restreints dans des compétences visant la gestion des activités administratives.

996 «Les altermondialistes» sont les partisans du mouvement politique qui s'opposent à la mondialisation. La mondialisation est un phénomène complexe d'interdépendance économique résultant des échanges de marchandises et de services et des flux de capitaux. (Voir en ce sens Robert CHARVIN, Relations internationales, droit et mondialisation, op.cit., p.29.)

997 SMOUTS (Marie-Claude), « Réflexions sur les méthodes de travail du Conseil de sécurité », op.cit., p.602.

998 Ibid. 
Ces compétences administratives dévolues aux organes restreints se présentent sous divers aspects. Nous avons les fonctions de préparation des réunions des divers organes et la rédaction des procès-verbaux de celles-ci, la réalisation des traductions, la fourniture d'informations, l'élaboration du projet de budget, l'entretien des locaux de l'organisation.

Le Conseil d’administration de l'OIT, à l'évidence, réalise pleinement ces fonctions administratives. Ces fonctions et ses pouvoirs sont les suivants:

1- il est l'organe exécutif de la Conférence générale ;

2- il coordonne les activités de l'organisation et veille à la bonne gestion de l'OIT ;

3- il prépare l'ordre du jour provisoire de la Conférence générale ;

4- il prépare le projet de budget à soumettre à la Conférence générale ;

5- il prépare et convoque les réunions aux Conférences internationales qui ont lieu à l'initiative et sous les auspices de l'OIT.

La Commission de l'UA ne fait pas exception à cette règle. Aux termes de l'Acte constitutif de l'UA la Commission :999

1- représente l'Union et défend ses intérêts ;

2- met en œuvre les décisions prises par les autres organes

3- organise et gère les réunions de l'Union

4- agit comme le dépositaire de l'Acte constitutif, de ses protocoles, des traités, des autres instruments juridiques et décisions adoptés par l'Union, et ceux hérités de l'OUA ;

5- prépare le budget et le programme de l'Union, pour approbation par les autres organes délibérants ;

6- gère les ressources budgétaires et financières, perçoit les recettes approuvées de différentes sources ;

7- applique les décisions de la Conférence relatives à l'ouverture et à la fermeture de sections et de bureaux administratifs ou techniques;

Relativement au Conseil d'administration du FMI, l'article XII section 3 alinéa (a) dispose qu’il «est responsable de la conduite générale du Fonds, et à cette fin, il exerce tous les pouvoirs que le Conseil des gouverneurs lui a délégués.» ${ }^{1000}$

Que faut-il déduire de la limitation des compétences des organes restreints à des fonctions purement administratives?

999 Voir l'article 3 de l'Acte constitutif de l'UA. Il est important de souligner que des fonctions politico-diplomatiques sont reconnues à la Commission. A ce titre, " elle élabore des plans stratégiques et des études ; prend certaines mesures dans certains domaines de responsabilités comme la lutte contre les pandémies, la gestion des catastrophes, la lutte contre la criminalité internationale ou encore l'intégration économique...D’autres fonctions politiques et diplomatiques portent sur la promotion de l'intégration et l développement socio-économique ou encore le renforcement de la coopération entre les États membres et la coordination de leurs activités dans les domaines d'intérêt commun. » (Voir Guy MVELLE, l'Union africaine, fondements, organes, programmes et actions, L'harmattan, Paris, 2007, p.219-220.)

1000 Voir l'article XII, Section 3 alinéa (a) du Statut du FMI. 
A cette question, le Professeur René-Jean DUPUY donne une approche satisfaisante. En effet, selon lui «dans les organisations on est parti d'un système qui, rassemblant des États qui se prétendent souverains en dépit de leur association, concentre le pouvoir suprême dans lorgane plénier interétatique.» ${ }^{1001}$

Les États membres ont voulu concentrer les pouvoirs politiques dans les organes pléniers où tous les États sont représentés sur un pied d'égalité. De ce point de vue, la définition des pouvoirs politiques important revient aux organes pléniers qui représentent l'ensemble des États membres. Quant aux organes restreints, qui en réalité reçoivent une délégation de pouvoirs des États membres ; ${ }^{1002}$ ils ne sont compétents seulement pour exécuter la politique générale définie par les organes pléniers.

A l'analyse, cette façon de procéder s'explique aisément au plan politique. Il serait contraire aux exigences démocratiques de laisser la latitude aux organes restreints qui sont composés de quelques membres de l'organisation de définir l'orientation politique d'une organisation internationale. Au plan juridique, elle vise à réduire les pouvoirs des organes restreints. Mais, cette limitation des pouvoirs des organes restreints est plus accentuée du fait de leurs compétences essentiellement spécialisées.

\section{B: DES COMPETENCES ESSENTIELLEMENT SPECIALISEES}

Dans létude du droit des organisations internationales, il est important d’opérer une distinction fondamentale entre organes généraux et organes spécialisés. Les organes généraux sont ceux dont les attributions coïncident avec celles de l'organisation. ${ }^{1003}$ Ce sont les organes pléniers. En revanche, les organes spécialisés se caractérisent par le fait qu'ils jouissent d’attributions partielles. Dans cette seconde catégorie, se rangent les organes restreints. ${ }^{1004}$

Cette spécialisation des compétences des organes restreints vise à leur confier une responsabilité particulière dans l'exécution d’une mission précise au sein de l'organisation. ${ }^{1005}$ Dans le fond, elle a pour finalité une limitation de leurs compétences. ${ }^{1006}$ Il revient aux organes

1001 DUPUY (René-Jean), « Le droit des relations entre les organisations internationales », RCADI, 1960-II, p.502.

1002 Cette délégation de pouvoirs des États membres aux organes restreints est formellement prévue par la plupart des Chartes constitutives. L'analyse de celles-ci révèle que dans la majorité des organisations internationales, les organes restreints agissent au nom et pour le compte des organes pléniers.

1003 QUOC DINH (Nguyen), Droit international public, op.cit.,p.615.

1004 Le Professeur Nguyen QUOC DINH relativise cette distinction en montrant que dans certaines organisations internationales, les organes restreints peuvent recevoir des compétences générales au même titre que les organes pléniers. Ainsi, affirme-t-il que «le Conseil de la S.D.N, organe restreint, avait reçu une compétence aussi générale que celle de l'Assemblée, organe plénier. De nos jours, les organes exécutifs de la plupart des institutions spécialisées sont des organes à compétence générale. » (Voir Nguyen QUOC DINH, Droit international public, LGDJ, $6^{\circ}$ édition, p.615.)

1005 YAO-NDRE (Paul), Droit des organisations internationales, op.cit., p.59.

1006 QUOC DINH (Nguyen), Droit international public, op.cit.,p.615. 
à compétence générale la mission de définir la politique générale de l’organisation. ${ }^{1007}$ Quant aux organes spécialisés qui sont généralement des organes restreints, ils jouissent de compétences particularisées. ${ }^{1008}$ Celles-ci, par nature sont partielles et limitées à certains objectifs de l’organisation.

A ce sujet, la Charte de l'ONU constitue un exemple. Les organes restreints que sont le Conseil de sécurité et le Conseil économique et social ont des compétences partielles au regard des buts et objectifs de cette organisation. La spécialisation du Conseil de sécurité apparaît clairement au Chapitre VI et VII de la Charte des Nations Unies. Aux termes du Chapitre VI, le Conseil est l'organe compétent pour « le règlement pacifique des différents. » ${ }^{1009}$ Quant au Chapitre VII, il est relatif aux actions que le Conseil peut prendre en cas de «menace à la paix, rupture de la paix et d'acte d'agression ».

Dans lesprit de la Charte, en cas de crise mettant en danger la paix et la sécurité internationale ou d'une situation susceptible d'y conduire, le Conseil peut intervenir de deux façons différentes : soit favoriser un règlement pacifique des différends (Chapitre VI), soit prendre des mesures nécessaires pour mettre fin à une menace contre la paix, une rupture de la paix ou un acte d'agression (Chapitre VII), ou même simplement pour faire exécuter un arrêt de la CIJ. ${ }^{1010}$ Les compétences du Conseil de sécurité sont donc très bien spécifiées par la Charte. Toutefois, cette spécialisation des compétences du Conseil de sécurité ne saurait être analysée comme une limitation de ses pouvoirs par l'Assemblée générale. Dans la répartition des pouvoirs au sein de l'ONU, le Conseil de sécurité demeure autonome vis-à-vis de l’Assemblée générale.

Ensuite quant au Conseil économique et social, il est chargé particulièrement de toutes les questions concernant la coopération économique et sociale internationale ainsi que de toutes les questions en relation avec les institutions spécialisées. ${ }^{1011}$ Le contenu des compétences spécialisées du Conseil économique et social est clairement précisé dans l’article 62, paragraphe 1 de la Charte des Nations Unies. ${ }^{1012}$ Ce sont entre autres les domaines, économique, social, de la culture intellectuelle et de léducation, de la santé publique et d'autres domaines connexes. Comme il est loisible de le constater, cet effort de spécialisation correspond à une volonté politique. Les États membres de l'organisation ouvrent au Conseil un champ de compétence pratiquement indéfini : la coopération économique et sociale à l'échelle universelle. ${ }^{1013}$ Cependant, il faut préciser que «ce que la compétence du Conseil gagne en étendue, elle le perd en profondeur.» ${ }^{1014}$ En réalité, le Conseil n’aucun pouvoir de décider la solution des problèmes économiques et sociaux internationaux ; il

1007 MONACO (Riccardo), « Les principes régissant les structures et le fonctionnement des organisations internationales », op.cit.,p.112.

1008 YAO-N'DRE (Paul), Droit des organisations internationales, op.cit., p.63.

1009 Le Chapitre VI de la Charte commence à l’article 33 et s'achève par l’article 38 de la Charte.

1010 VIRALLY (Michel), Lorganisation mondiale, op.cit., p.108.

1011 BEN ACHOUR (Rafâa), Institutions de la société internationale, op.cit., p.196.

1012 Voir l'article 62, paragraphe 1 de la charte de l'ONU.

1013 RUCZ (Claude), "Commentaire de l'article 62 de la Charte des Nations Unies », in La Charte des Nations Unies, commentaire article par article », op.cit., p.953 et 954.

1014 Ibid. 
n’est habilité quà provoquer des études et adresser des recommandations à l’Assemblée générale et aux institutions spécialisées. ${ }^{1015}$

L’Assemblée générale à travers cette spécialisation fonctionnelle du Conseil économique et social a limité substantiellement ses pouvoirs. Sa compétence ne connaît pas d’autre borne que la volonté de l'Assemblée générale : en répartissant les compétences entre les organes de l'ONU, elle cantonne le champ d'action du Conseil et détermine les fonctions attendues de lui. ${ }^{1016}$

Relativement au Conseil de paix et de sécurité, il est nécessaire d'inscrire ses pouvoirs dans le cadre général des objectifs poursuivis par l'UA. Dans cette perspective, il faut relever que l'UA est une organisation généraliste, ${ }^{1017}$ c'est-à-dire une organisation internationale qui s'engage à poursuivre des activités dans tous les domaines touchant à la vie des États et des peuples. ${ }^{1018}$ Ainsi, les États membres entendaient coordonner et harmoniser leurs politiques générales dans presque tous les domaines : la politique et la diplomatie, léconomie, les transports et les télécommunications ; l'éducation et la culture ; la santé, l'hygiène et la nutrition ; la science et la technique ; et enfin la défense et la sécurité. ${ }^{1019}$

Dans cette panoplie d’objectifs assignés à la nouvelle organisation panafricaine, les membres fondateurs ont créé le Conseil de paix et de sécurité en vue de mettre en place une structure opérationnelle pour la mise en œuvre efficace des décisions prises dans le domaine de la prévention des conflits et du rétablissement de la paix. ${ }^{1020} \mathrm{Il}$ en résulte que les membres fondateurs ont limité substantiellement les attributions du Conseil de paix et de sécurité en le confinant exclusivement dans le domaine de la paix et la sécurité.

Aux termes de l'article 6 du Protocole relatif à sa création, ${ }^{1021}$ il est chargé de :

- la promotion de la paix, de la sécurité et de la stabilité en Afrique

- l'alerte rapide et de la diplomatie préventive

- le rétablissement de la paix

- la conduite des opérations d’appui à la paix

La Conférence des Chefs d'État et de gouvernement, l'organe plénier de l'UA a voulu se réserver les pouvoirs généraux, c’est-à-dire les pouvoirs qui coïncident avec ceux de l'organisation,

1015 Ibid.

1016 Ibid,p.953 et 954 .

1017 MVELLE (Guy), L'Union africaine, fondements, organes, programmes et actions, L'Harmattan, Paris, 2007, p.92.

1018 Ibid.

1019 Ibid, p.93.

1020 Ibid, p.178.

1021 Voir l'article 6 du Protocole relatif à la création du Conseil de paix et de sécurité de l'Union africaine. 
au détriment du Conseil de paix et de sécurité, qui est l'organe restreint. ${ }^{1022}$ De ce fait, ce sont les Chefs d’État et de gouvernement qui définissent la politique générale de lorganisation.

En définitive, les organes pléniers limitent le statut des organes restreints par le pouvoir de désignation de ces membres et par la réduction de leurs compétences. Ce qui contribue à la subordination des organes restreints aux organes pléniers. Mais, cette subordination est encore plus accentuée par le contrôle exercé par les organes pléniers sur les organes restreints.

\section{SECTION II : LE CONTRÔLE DES ORGANES RESTREINTS PAR LES ORGANES PLENIERS}

Les organes restreints dans la plupart des organisations internationales sont subordonnés aux organes pléniers. Le corollaire de cette subordination est le pouvoir de contrôle reconnu aux organes pléniers sur les organes restreints. Ce contrôle se réalise par le pouvoir hiérarchique exercé par les organes pléniers sur les organes restreints (Paragraphe I). Cependant, il faut souligner que le Conseil de sécurité déroge à cette règle dans la mesure où il ne fait l'objet d'aucun contrôle de l’Assemblée générale (Paragraphe II).

\section{PARAGRAPHE I : LE POUVOIR HIERARCHIQUE DES ORGANES PLENIERS SUR LES ORGANES RESTREINTS}

Au sens du droit interne, le pouvoir hiérarchique doit s'entendre du contrôle exercé par le supérieur hiérarchique sur les actes des organes subordonnés. Dans les organisations internationales, ce pouvoir hiérarchique peut exister entre les organes pléniers et les organes restreints.

Les organes pléniers des organisations internationales peuvent se trouver dans une situation de supériorité hiérarchique par rapport aux organes restreints. Cette supériorité hiérarchique implique l'exercice d'un pouvoir hiérarchique des organes pléniers sur les organes restreints. Dans les faits, ce pouvoir hiérarchique s'exerce par le pouvoir de substitution (A) et d'évocation des organes pléniers sur les organes restreints (B).

1022 Pour preuve, aux l'article 9 de l'acte constitutif de l'UA, les pouvoirs et attributions de la Conférence sont les suivants : -définir les politiques communes de l'Union -recevoir, examiner et prendre toutes les décisions sur les rapports et les recommandations des autres Organes de l'Union et prendre des décisions à ce sujet -Assurer le contrôle de la mise en œuvre des politiques et décisions de l’Union, veiller à leur Application par tous les États membres ( ...). 


\section{A : LE POUVOIR DE SUBSTITUTION DES ORGANES PLENIERS AUX ORGANES RESTREINTS}

Le pouvoir de substitution des organes pléniers sur les organes restreints est l'expression du lien de subordination qui existe entre ces deux organes.

Il signifie que l'organe supérieur a le pouvoir de se substituer, en cas d'inertie, à l'organe inférieur qui aurait manqué à son devoir d’exercer une compétence déterminée. ${ }^{1023}$ De cette approche, il faut relever que la mise en œuvre du pouvoir de substitution est conditionnée par un disfonctionnement de l'organe inférieur qui n'exerce pas les compétences qui lui sont reconnues par la Charte constitutive. Dans ces conditions, l'organe supérieur est habilité, au nom du pouvoir de substitution, à agir en lieu et place de l'organe inférieur.

En pratique, la résolution Dean Acheson (377 V) -que nous avons évoqué- était une application du pouvoir de substitution de l'Assemblée générale des Nations Unies sur le Conseil de sécurité. En effet, il n’est pas inutile de rappeler que la résolution $377(\mathrm{~V})$ reconnaît à l’Assemblée générale le droit de suppléer le Conseil de sécurité lorsque ce dernier, paralysé par le veto «manque à s'acquitter de sa responsabilité principale. » ${ }^{1024}$

Cette résolution exprime une volonté politique de l'Assemblée générale de se substituer au Conseil de sécurité. Mais en réalité, la résolution Dean Acheson est un instrument utilisé principalement par les États-Unis qui disposait d'une " majorité automatique " à l'Assemblée, pour contourner les vetos soviétiques au Conseil de sécurité. Mais, juridiquement le recours à cette résolution ne traduit pas un quelconque pouvoir hiérarchique de l'Assemblée générale sur le Conseil de sécurité, mais plutôt une volonté politique des États-Unis de déplacer le pouvoir de décision en matière de maintien de la paix de la sécurité internationale à l'Assemblée générale.

En revanche, dans le fonctionnement de l'UA il est facile de constater que la substitution de la Conférence des Chefs d'État et de gouvernement à la Commission et au Conseil de paix et de sécurité s’analyse comme la mise en ouvre d’un pouvoir hiérarchique. En général, la Conférence des Chefs d'État et de gouvernement se substitue à ces deux organes restreints lorsqu'il s'agit des questions politiques fondamentales pour la paix et la sécurité du continent.

La position prééminente des Chefs d'État au sein de l'UA se manifeste dans les actions en vue de l'apaisement des litiges qui demeurent l’ouvre personnelle des Chefs d'État agissant collectivement ou individuellement. ${ }^{1025}$ Les Chefs d'État dominant de leur stature l'organisation, il est donc évident que la Conférence des Chefs d'État et de gouvernement se substitue à la Commission et au Conseil de paix et de sécurité. Mais, il faut reconnaître que cette situation entraîne des difficultés dans le fonctionnement de l'organisation.

1023 MONACO (Riccardo), «Les principes régissant les structures et le fonctionnement des organisations internationales », op.cit, p.114.

1024 DEGNI-SEGUI (René), « Commentaire de l’article 24 de la Charte des Nations Unies », op.cit., p.452.

1025 GONIDEC (Pierre-François), L'OUA trente ans après, Karthala, 1993, p.29 et 30. 
Le Professeur Koffi AHADZI estime que «les pouvoirs reconnus au Président de la Commission en matière de paix et de sécurité crée des frictions avec les Chefs des États membres, et particulièrement le Président de la Conférence, qui veulent, comme du temps de l'ex-OUA, continuer à occuper seuls le devant de la scène et cantonner le Président de la Commission dans un rôle subalterne de fonctionnaire exécutant servilement leur volonté.» ${ }^{1026}$ Dans les faits, «le désaveu public infligé par le Président en exercice OBASSANDJO à Monsieur KONARE à propos de la décision prise par celui-ci, conformément à l'article 10 du protocole portant création du Conseil de paix et de sécurité, et sur habilitation expresse de celui-ci, de nommer un médiateur pour aider à résoudre la crise togolaise née des élections d’avril 2005, montre bien que les Chefs d'État entendent conserver le leadership de l'Union et réduire à peu de chose la marge de manœuvre et les pouvoirs effectifs du Président de la Commission.» ${ }^{1027}$ Ce faisant, renchérit le Professeur AHADZI, «lon court le risque de voir renaître l'Ex-OUA de ses cendres et de condamner l'UA à être ballotée, telle une girouette, au gré de la volonté des Chefs d'États africains.» ${ }^{1028}$

Le pouvoir de substitution des organes pléniers est une expression de leur supériorité juridique sur les organes restreints. C'est dans cette même logique que s'inscrit le pouvoir d'évocation des organes pléniers sur les organes restreints.

\section{B : LE POUVOIR D’EVOCATION DES ORGANES PLENIERS SUR LES ORGANES RESTREINTS}

Le pouvoir d'évocation est aussi une expression du pouvoir hiérarchique des organes pléniers sur les organes restreints. Il s’entend comme le principe en vertu duquel certaines activités ou compétences sont assumées par l'organe supérieur, qui les soustrait à l'organe inférieur auquel elles reviennent normalement. ${ }^{1029} \mathrm{~A}$ la différence du pouvoir de substitution, sa mise en œuvre par l’organe plénier n'est pas généralement conditionnée par une défaillance de l’organe restreint.

Pris dans cette acception, les organes pléniers exercent directement des compétences reconnues expressément par la Charte constitutive aux organes restreints. Ce faisant, lorgane plénier pénètre directement dans la sphère de compétence de l’organe restreint, montrant ainsi sa supériorité hiérarchique.

En pratique, dans le fonctionnement des organes des Nations Unies, l'Assemblée générale dispose d'un pouvoir d'évocation qui lui permet de décider de la conclusion d'accords de liaison

1026 AHADZI (Koffi), « Réflexions critiques sur l’Union africaine », op.cit., p.100. Larticle 10 du Protocole relatif à la création dispose que « le Président de la commission, sous l’autorité du Conseil de paix et de sécurité et en consultation avec toutes les parties impliquées dans un conflit, déploie tous les efforts et prend toutes les initiatives jugées appropriées en vue de la prévention, de la gestion et du règlement des conflits. »

1027 Ibid.

1028 Ibid.

1029 MONACO (Riccardo), « Les principes régissant la structure et le fonctionnement des organisations internationales », op.cit., p.114. 
qui rentrent dans la compétence du Conseil économique et social. ${ }^{1030}$ Dans l'esprit des auteurs de la Charte, le Conseil économique et social devait particulièrement se charger de la coordination avec les autres organisations internationales et avec les institutions spécialisées, prévues à l’article 57. En d’autres termes, le Conseil économique et social est le centre moteur du système de coordination avec les institutions spécialisées et les autres organisations internationales selon de la charte des Nations Unies. ${ }^{1031}$

Cependant, dans la pratique le Conseil économique et social s'est trouvé souvent écarté par l'Assemblée générale de certains accords comme avec la SDN ou avec le Conseil de l'Europe. ${ }^{1032}$ L’Assemblée générale néprouve aucune gêne à agir avec cette liberté dans la mesure ${ }^{1033}$ où elle peut conclure une Convention avec une organisation internationale ou une institution spécialisée, sans passer par l'intermédiaire du Conseil économique et social. Cette pratique de l'Assemblée générale est politiquement irréprochable en ce sens qu’elle a délégué ses compétences en la matière au Conseil économique et social.

De même, dans la distribution des pouvoirs au FMI, le Conseil des gouverneurs jouit d'un pouvoir dévocation sur le Conseil d'administration. Aux termes de l'article XII, section $2 \mathrm{du}$ Statut du FMI, le Conseil des gouverneurs est l'organe investit de tous les pouvoirs. Aussi, peut-il déléguer certains de ses pouvoirs au Conseil d'administration. ${ }^{1034}$ Sur cette base, juridiquement le Conseil des gouverneurs peut exercer son pouvoir d'évocation sur le Conseil d’administration.

Dans le fonctionnement interne des organisations internationales, les pouvoirs des organes restreints sont considérablement limités par le pouvoir hiérarchique des organes pléniers. Cependant, cette limitation des pouvoirs des organes restreints par les organes pléniers connait une exception avec le Conseil de sécurité qui n’est pas soumis au pouvoir de contrôle de l’Assemblée générale.

\section{PARAGRAPHE II : L'EXCEPTION DU CONSEIL DE SECURITE DES NATIONS UNIES}

Le Conseil de sécurité des Nations Unies se distingue considérablement des autres organes restreints des organisations internationales. Ce particularisme découle en grande partie de ses pouvoirs qui sont loin de caractériser le pouvoir conférer aux organisations internationales. ${ }^{1035}$ En vérité, ces pouvoirs sont si exagérément étendus (A) qu’ils ont une nature quasi-législative (B).

1030 DUPUY (René-Jean), « Le droit des relations entre les organisations internationales », RCADI, 1960-II, p.510.

1031 Ibid.

1032 Ibid.

1033 Ibid.

1034 DIEZ DE VELASCO VALLEJO (Manuel), Les organisations internationales, op.cit., p.395.

1035 DEGNI-SEGUI (René), "Commentaire de l'article 24 paragraphe 1et 2 de la Charte des Nations Unies », op. cit., p.460. 


\section{A : DES POUVOIRS EXAGEREMENT ETENDUS}

De par son statut d’organe restreint, le Conseil de sécurité bénéficie de compétences déléguées découlant de la volonté politique de l’ensemble des membres siégeant à l'Assemblée générale. De ce fait, les pouvoirs du Conseil de sécurité devraient être définis par l'Assemblée générale dans une proportion assez raisonnable.

Mais au regard du contenu de la Charte, les pouvoirs du Conseil de sécurité ont été exagérément étendus par les fondateurs de l’organisation. ${ }^{1036}$ A ce sujet, le Professeur DEGNI-SEGUI relève que «le Conseil de sécurité dispose de pouvoirs spécifiques et de pouvoirs généraux.» ${ }^{1037}$ Les pouvoirs spécifiques, précise-t-il- sont ceux qui sont expressément prévus par l'article 24, paragraphe 2 de la Charte. Dans ce domaine précis, le Conseil de sécurité jouit d’un certain nombre de pouvoirs énumérés aux Chapitres VI (règlement pacifique des différends), VII (actions en cas de menace contre la paix, de rupture de la paix et d'acte d'agression), VIII (accords régionaux) et XII (régime international de tutelle).

Le Conseil de sécurité jouit d'une compétence très large dans le domaine du maintien de la paix et de la sécurité internationales. Or, ce domaine constitue le premier but des Nations Unies, ${ }^{1038}$ mieux, il s'agit d’un objectif que l’on peut qualifier de fondamental pour la communauté internationale. ${ }^{1039}$ Ainsi pour l'atteindre, l'article 24 fait du Conseil de sécurité la pierre angulaire du système de sécurité collective établie par la Charte en lui confiant la responsabilité principale. ${ }^{1040}$

Cependant, pour comprendre l'intention réelle des fondateurs de l'organisation, il faut remonter à Conférence de Yalta qui a donné naissance aux Nations Unies. Vainqueurs de la deuxième guerre mondiale, l'intention des cinq membres permanents était de fonder un véritable pouvoir international, c'est-à-dire en pratique, de maintenir la direction du temps de la guerre. ${ }^{1041}$

1036 Dans la doctrine, il est généralement admis que les pouvoirs du Conseil de sécurité sont relativement étendus en raison du droit de veto des membres permanents. En réalité, le droit de veto n’enlève absolument rien aux pouvoirs illimités que les grandes puissances ont voulu se donner dans la Charte avec le conseil de sécurité. En vérité, comme l'a signifié le Professeur Charles CHAUMONT, le droit de veto « est fait pour réunir, devant témoins, les représentants des maîtres du monde, afin que chacun sache à quoi sén tenir sur les autres et qu'en soient déduites les possibilités d’agir. " (Voir SOUBEYROL (Jacques), "Considérations sur la crise financière de l'ONU ", RGDIP, 1967, p.434). Ainsi, il faut savoir que dans son essence, les grandes puissances ont prévu le droit de veto au Conseil de sécurité uniquement pour empêcher une décision arbitraire de l'une d'entres elles allant à l'encontre des intérêts des autres membres permanents. Cela dit, il n’a pas été prévu surtout à l'intention des membres non permanents, qui ne constituent aucune menace réelle pour les grandes puissances, mais pour équilibrer les rapports de force entres les grandes puissances dans la mesure où pour les questions de fonds il est exigé absolument une unanimité entre eux.

1037 DEGNI-SEGUI (René), "Commentaire de l'article 24 paragraphe I de la Charte des Nations Unies », op.cit., p.458 à 460 .

1038 MANUEL DIEZ DE VELASCO (Vallejo), Les organisations internationales, op.cit., p.213.

1039 Ibid.

1040 DEGNI-SEGUI (René), « Commentaire de l'article 24 paragraphe 1 de la Charte des Nations Unies», op.cit., p.447.

1041 ZORGBIBE (Charles), Les organisations internationales, Que sais-je ? n 8855, PUF, p.22. 
Pour les grandes puissances, «il ne s’agit plus de sombrer dans le juridisme, mais d’entreprendre une action réaliste ; l'heure nest plus à la constitution d'un tribunal des nations mais la mise en place d'une gendarmerie internationale.» ${ }^{1042}$ De ce fait, concentrant tous les pouvoirs en matière de maintien de la paix, le Conseil de sécurité institutionnalise le pouvoir des «grands», des cinq membres permanents. Si une intervention militaire s'avère nécessaire, c'est le Comité d'état-major composé de représentants des cinq «grands» qui sera chargé de son exécution technique. ${ }^{1043}$

Les pouvoirs du Conseil de sécurité ne se limitent pas exclusivement aux pouvoirs spécifiques. Au-delà de ceux-ci, il jouit aussi de pouvoirs généraux. Il faut le rappeler, les pouvoirs généraux sont ceux qui se confondent avec ceux de l'organisation. Comme le souligne le Professeur Nguyen QUOC DINH : «si les attributions du Conseil de sécurité sont limitées aux questions du maintien de la paix, ceci suffit à en faire un organe général, du fait de létendue et de la portée politique de ce domaine d'action.» ${ }^{1044} \mathrm{Il}$ en résulte que l'attribution au Conseil de sécurité des pouvoirs dans un domaine aussi important que le maintien de la paix et de la sécurité fait de lui un organe incontournable dans la mise en œuvre de la politique des Nations Unies.

Mais au-delà des pouvoirs découlant directement de la Charte, de plus en plus, nous assistons à un élargissement de ses pouvoirs dans la mesure où les causes de rupture de la paix ne se limitent plus aux conflits armés. Le Conseil lui-même a reconnu parmi les causes de menace à la paix et à la sécurité internationales : le sous-développement, le courant massif des réfugiés à la suite d'une guerre civile, les violations généralisées des droits de l'homme, le terrorisme international. ${ }^{1045}$ Le Président du Conseil de sécurité - dans sa séance du 31 janvier 1992- souligne que «la paix et la sécurité internationales ne découlent pas seulement de l'absence de guerres et de conflits armés. D’autres menaces de nature non militaire à la paix et à la sécurité trouvent leur source dans l'instabilité qui existe dans les domaines économique, social, humanitaire et écologique.» ${ }^{1046}$ Le Conseil n’est pas cantonné dans les questions purement politiques, mais son domaine sétend dans le domaine économique, social et humanitaire.

Il ne faut pas perdre de vue le pouvoir de décision du Conseil de sécurité prévu à l’article 25 de la Charte : «les membres de l'organisation conviennent d’accepter et d’appliquer les décisions

1042 Ibid.

1043 En pratique, précise le Professeur Robert CHARVIN « le Conseil de sécurité pratique la technique de la délégation confiant à certaines grandes puissances la mission d’agir militairement pour le compte des Nations Unies, qui, en fait, ne maîtrisent plus par la suite la situation, ainsi que l'a dénoncé le Secrétaire général des Nations Unies Boutros Ghali lui-même. Cette délégation est une violation de l'esprit de la Charte qui est de retirer aux États la charge des opérations de police internationale : la pratique est, au contraire, le retour au " dédoublement fonctionnel » traditionnel des grandes puissances juges et parties dans le maintien de l'ordre international. » (Voir Robert CHARVIN, Relations internationales, droit et mondialisation, un monde en sens unique, op.cit.,p.152-153).

1044 QUOC DINH (Nguyen), Droit international public, op.cit., p.615.

1045 GAJA (Giorgio), " Réflexions sur le rôle du Conseil de sécurité dans le nouvel mondial », op.cit., p.302.

1046 Ibid. 
du Conseil de sécurité conformément à la présente Charte.» ${ }^{1047}$ Le but évident de l'article 25 est de mettre en pratique l’engagement des États membres de conférer au Conseil de sécurité la responsabilité principale du maintien de la paix et de la sécurité internationale. ${ }^{1048}$

Le Conseil de sécurité est un type particulier d’organe restreint en raison de ses pouvoirs juridiques démesurément étendus qui s’inscrivent dans la logique hégémonique des grandes puissances. Qui plus est, la singularité de ses pouvoirs donne de constater qu'ils surpassent ceux de l’Assemblée générale.

Dans le système des Nations Unies, la recherche d'un équilibre n’apparaît réellement qu’entre ces deux organes politiques que constituent l'Assemblée générale et le Conseil de sécurité. ${ }^{1049}$ Toutefois au-delà de la distribution formelle des pouvoirs entre ces deux organes politiques, ${ }^{1050} \mathrm{il} \mathrm{est}$ nécessaire de s'interroger si les fondateurs de l'organisation avaient réellement et sincèrement été animés d'un souci d'équilibre entre ceux-ci ?

Au regard des dispositions de la Charte des Nations Unies, le constat est clair : le Conseil de sécurité se présente comme un organe restreint aristocratique. ${ }^{1051}$ Ainsi, dans le domaine du maintien de la paix qui constitue comme l'a souligné le Professeur DEGNI-SEGUI «lobjectif primordial que vise l'ONU et le principe cardinal sur lequel repose tout l'édifice onusien», le Conseil de sécurité jouit de pouvoirs sans commune mesure avec ceux de l’Assemblée générale. ${ }^{1052}$ S’il est vrai que l'Assemblée générale et le Conseil de sécurité peuvent agir dans le cadre du Chapitre VI par la voie de recommandation sans aucune valeur contraignante pour les États, il faut souligner que relativement au Chapitre VII, le Conseil de sécurité dispose de pouvoir de décision ayant un caractère obligatoire. ${ }^{1053}$ Le Conseil de sécurité est donc le seul organe de l'ONU dont les décisions s’imposent aux États.

Pour conforter le Conseil dans sa position d'organe restreint ayant un pouvoir obligatoire vis-à-vis des États, un arsenal de dispositions juridiques a été prévu dans la Charte. Il faut mentionner l'article 2, paragraphe $2^{1054}$ selon lequel « les États doivent remplir de bonne foi leurs obligations.» La

1047 Voir l'article 25 de la Charte des Nations Unies.

1048 SUY (Erik), "Commentaire de l'article 25 de la Charte des Nations Unies ", in La Charte des Nations Unies, commentaire article par article, op.cit., p.477.

1049 CHAUMONT (Charles), "L'équilibre des organes politiques des Nations Unies et la crise de lorganisation », op.cit., p.429.

1050 La Charte a institué un équilibre politique afin de garantir l’universalité et la continuité de l'organisation, en ce sens que la représentation universelle incombe à l'Assemblée générale et le respect des intérêts particuliers des grandes puissances au Conseil de sécurité.

1051 CAVARE (Louis), Le droit international public positif, Tome I, Pédone, Paris, p.1973.

1052 Dans le cadre du maintien de la paix, il faut faire la distinction entre le règlement pacifique des différends prévu au chapitre VI de la Charte et l'action en cas de menace contre la paix, de rupture de la paix et d'acte d'agression prévu au Chapitre VII.

1053 A ce sujet, il convient de préciser que les résolutions prises dans le cadre du chapitre VII portent généralement la mention «exige » ou «décide».

1054 Voir l'article 2, paragraphe 2 de la Charte des Nations Unies. 
bonne foi qui exclut toute tentative de «fraude à la loi», toute ruse ${ }^{1055}$ exige l'obligation d'exécution honnête et scrupuleuse d'un engagement pris par un sujet de droit international, qu'il s'agisse d'un engagement conventionnel ou unilatéral. ${ }^{1056}$ Les États membres sont tenus d’appliquer les décisions, au nom du principe de la bonne foi et surtout de l'engagement pris à l'article 25 «d'accepter et d'appliquer les décisions du Conseil de sécurité» ${ }^{1057}$.

Larticle 48 de la Charte confirme cette volonté de conférer au Conseil de sécurité un pouvoir décisoire. Aux termes de cette disposition «les mesures nécessaires à l'exécution des décisions du Conseil de sécurité pour le maintien de la paix et de la sécurité internationales sont prises par tous les membres des Nations Unies ou certains d'entre eux, selon l'appréciation du Conseil.» ${ }^{1058}$ Cette disposition réaffirme aussi et à nouveau l’obligation d’exécuter les décisions du Conseil de sécurité. ${ }^{1059}$

Manifestement, toutes les mesures ont été prises dans la Charte afin de faire du Conseil de sécurité un organe décisoire contrairement à l’Assemblée générale qui ne peut que faire des recommandations aux États membres.

Un autre aspect assez important des pouvoirs du Conseil de sécurité et qui montre une fois encore sa prédominance sur l’Assemblée générale mérite dêtre souligné. Il s’agit du caractère discrétionnaire des attributions du Conseil de sécurité qui ne fait aucun doute. ${ }^{1060}$ Mais, concrètement que signifie le pouvoir discrétionnaire du Conseil de sécurité ? La notion de pouvoir discrétionnaire a fait l'objet de nombreuses études en droit administratif français. Il en découle que le pouvoir discrétionnaire « est essentiellement défini par défaut ou par confrontation avec lorgane chargé de le contrôler. Ce pouvoir est envisagé et défini par la marge d’appréciation que le juge soctroie dans un certain type de contrôle. Dès lors est discrétionnaire le pouvoir dont le contrôle par le juge est à un niveau d'intensité bas qui permet de le qualifier ainsi.» ${ }^{1061}$ Mais en droit international, cette approche connaît quelques évolutions. Le dictionnaire du droit international appréhende le pouvoir discrétionnaire « d'une manière très large comme le pouvoir reconnu à un

1055 LE JEUNE (Pierrick), Introduction au droit des relations internationales, op.cit., p.18

1056 Ibid.

1057 Voir l'article 25 de la charte des Nations Unies. Il faut souligner que la CIJ a pour sa part pris le soin de rappeler la portée de l'article 25 de la charte : «la Charte » affirme-t- elle dans son avis consultatif du 11avril 1949, «a défini la position des membres par rapport à lorganisation en lui prescrivant de lui donner pleine assistance dans toute action entreprise par elle, d'accepter et d’appliquer les décisions du Conseil de sécurité.»

1058 Voir l'article 48 de la Charte des Nations Unies.

1059 LANFRANCHI (Marie-Pierre), « La valeur juridique en France des résolutions de Conseil de sécurité », op.cit., p. 36.

1060 Plusieurs études ont été consacrées à ce point. Mais à titre indicatif, nous pouvons mentionner ici l’article du Professeur Giorgio GAJA intitulé «réflexion sur le rôle du Conseil de sécurité dans le nouvel ordre mondial », RGDIP, 1993, pp.297-319; Gérard COHEN JONATHAN, «Commentaire de l’article 39 de la Charte des Nations Unies » in La Charte des Nations Unies, Commentaire article par article, p.645-666 ; Rafâa BEN ACHOUR, Institutions de la société internationale, Centre de publication universitaire, Tunis, 2004, pp.19O-194.

1061 SOREL (Jean-Marc), " Le caractère discrétionnaire des pouvoirs du Conseil de sécurité : remarques sur quelques incertitudes partielles », in Arès, n55, volume XXI, Fascicule 3, mai 2005, p.14. 
sujet de droit ou à un organe qui n’est pas lié par une règle de droit, et dont par conséquent, l'usage n’a pas à être motivé et n'est pas susceptible de contrôle.» ${ }^{1062}$ Comme on peut bien le constater, contrairement à l'approche du droit administratif, celui du droit international exclut l'idée d'un quelconque contrôle du pouvoir discrétionnaire. Elle correspond à ce que les auteurs de la Charte des Nations Unies ont bien voulu des pouvoirs qu'ils ont reconnus au Conseil de sécurité : un pouvoir soustrait à tout contrôle juridictionnel. ${ }^{1063}$ Toutefois, le pouvoir du Conseil de sécurité n’est certainement pas arbitraire, si l’on entend par là que ses décisions prennent leur justification à une source véritablement objective extérieure à leur auteur. ${ }^{1064}$ Bien au contraire, le Conseil fonde ses décisions sur la Charte, particulièrement sur les dispositions de l'article 24.

Aux termes du Chapitre VII de la Charte, le Conseil de sécurité est le seul organe compétent en matière de maintien de la paix et de la sécurité internationales pour interpréter et qualifier les situations de rupture à la paix, d'agression, et de menace à la paix, puis de décider des mesures à prendre. ${ }^{1065} \mathrm{Il}$ en résulte que le Conseil de sécurité dans le domaine du Chapitre VII de la charte jouit de pouvoirs étendus, alors que l’Assemblée générale qui est la conscience collective de la société internationale de par son universalité même, ${ }^{1066}$ n’a qu'une fonction limitée à létude des questions se rattachant à la paix. ${ }^{1067}$

L’Assemblée générale devient l’organe auquel l’on a doté d’une sorte "d’autorité morale.» ${ }^{1068}$ En revanche, le Conseil de sécurité bénéficie de compétences exorbitantes, discrétionnaires et illimitées dans le domaine de la sécurité collective. Ce qui fait de lui naturellement la pièce maîtresse du système des Nations Unies conformément à la volonté des grandes puissances. Ces pouvoirs sont si étendus qu'il convient de s'interroger s'ils n’ont pas une nature quasi-législative?

\footnotetext{
1062 Ibid.

1063 Nous avons déjà montré dans nos analyses précédentes qu’aucun contrôle de constitutionnalité nétait possible par la Cour internationale de justice sur les actes du Conseil de sécurité.

1064 LAGRANGE (Evelyne), "Le Conseil de sécurité des Nations Unies peut-il violer le droit international ?", in Revue belge de droit international », Editions Bruylant, Bruxelles, 2004/2, p.569.

1065 Pour être plus précis, il est nécessaire de se référer aux dispositions des articles 39 à 54 de la Charte des Nations Unies.

1066 CHAUMONT (Charles), "Léquilibre des organes politiques des Nations Unies et la crise de l'organisation ", op.cit., p.432.

1067 Ibid, p.431.

1068 BENNANI (Mohamed), "Commentaire de l'article 10 de la Charte des Nations Unies », in La Charte des Na-

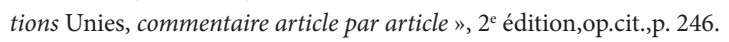




\section{B: DES POUVOIRS QUASI-LEGISLATIFS}

Avant tout propos, il convient délucider brièvement la notion de pouvoir législatif. La notion de pouvoir législatif fait référence à la fonction du parlement dans l'ordre interne étatique. De fait, le pouvoir du parlement est de légiférer, c'est-à-dire produire des normes ayant une valeur de loi. La loi s'entend au sens large comme une norme juridique ayant un caractère général, impersonnel et permanent. Cette précision faite, il est important de poser le problème du pouvoir législatif en relation avec le Conseil de sécurité des Nations Unies. La question est donc posée de savoir si la Charte de San Francisco s'accommode de la fonction législative du Conseil de sécurité se déclinant en l'adoption de normes générales, permanentes et contraignantes ? ${ }^{1069}$ La question apparaît provocante au juriste classique à qui l'on a enseigné qu'au nombre des caractéristiques essentielles de la société internationale et du droit international figure précisément l’absence de législateur international. ${ }^{1070}$

La réponse à cette question n'est pas aisée. L’analyse des pouvoirs du Conseil de sécurité donne de constater que ceux-ci semblent sorienter dans une perspective quasi-législative. Le Conseil de sécurité a pris ces dernières années des résolutions qui peuvent être qualifiées de normes particulières et générales. ${ }^{1071}$ Depuis quelques années, on a pu constater une tendance de cette institution à se comporter en véritable «législateur», une tendance qui s'est accélérée après la fin de la guerre froide puis, plus encore après le 11 septembre 2001 et l'avènement de la «guerre contre le terrorisme.» ${ }^{1072}$

Cette propension du Conseil de sécurité à sériger en «législateur» se matérialise par le contenu de ses résolutions. De ce point de vue ses résolutions se singularisent par leur caractère général et permanent. La preuve est apportée par la résolution 1373 (2001) adoptée à l'unanimité des États membres du Conseil de sécurité. ${ }^{1073}$ Dans cette résolution, le Conseil de sécurité décide que tous les États ont une obligation de prévention et de répression du terrorisme. Ils doivent prendre des dispositions nationales incriminant la fourniture ou la collecte délibérée par les nationaux sur leur territoire des fonds destinés au terrorisme. Ces mesures comportent également le gel des fonds ou avoirs financiers, voire des ressources économiques liées aux activités terroristes. ${ }^{1074}$ Manifestement, la résolution 1373 apparaît comme « la loi anti-terroriste » sur la planète terre. ${ }^{1075}$ Elle fixe des normes générales, impersonnelles destinées à s’appliquer aux États membres et non

1069 KOKOROKO (Komla Dodzi), « Réflexions sur le pouvoir législatif du Conseil de sécurité de l'ONU », in RBSJA, $\mathrm{n}^{\circ} 19$, décembre 2007, p.125.

1070 TERCINET (Josiane), «Le pouvoir normatif du Conseil de sécurité : Le Conseil de sécurité peut-il légiférer ? ", in Revue belge de droit international, Editions Bruylant, Bruxelles, 2004/ 2, p.528.

1071 Ibid.

1072 Ibid.

1073 Ibid.

1074 Voir la résolution 1373 (2001) du Conseil de sécurité.

1075 KOKOROKO (Komla Dodzi), « Réflexion sur le pouvoir législatif du Conseil de sécurité de l'ONU », op.cit., p.128. 
membres de l'ONU. La référence faite par cette résolution au Chapitre VII de la Charte des Nations Unies la rend obligatoire pour tous les États. ${ }^{1076}$ C’est ici, d’une véritable législation internationale qu'il s'agit. ${ }^{1077}$

La posture de «législateur international» du Conseil de sécurité ${ }^{1078}$ se justifie à plusieurs égards. Au regard de la théorie des compétences implicites qui permet aux organisations internationales d'intervenir dans les domaines que le traité de base nénonce pas expressément. ${ }^{1079}$ La fonction législative du Conseil de sécurité témoignerait des adaptations, des accommodements et d'une certaine façon des déformations qu'impose toujours lépreuve du réel dans la mesure où un système de sécurité d’une communauté internationale en perpétuelle évolution ne pourrait fonctionner efficacement en demeurant totalement fidèle au schéma initial. ${ }^{1080}$ Sous cet angle, les attentats du 11 septembre 2001 montrent clairement la nécessité d'adapter le Conseil de sécurité aux nouveaux types de menaces et défis apparus dans les relations internationales. A ce sujet, le Professeur KOKOROKO fait observer que, «l'agresseur n'est pas un État, l'agression n'est pas une attaque militaire, le théâtre des opérations n’est pas défini géographiquement.» ${ }^{1081}$ Dans ces conditions, souligne-t-il, «lamélioration du pouvoir de décision passerait par la reconnaissance au Conseil de sécurité d'un pouvoir large de qualification des faits entrant dans sa sphère de compétence sur le fondement de l'article 39.» ${ }^{1082}$

Un deuxième argument non moins négligeable peut être invoqué comme fondement de la fonction législative du Conseil de sécurité. Il sagit cette fois-ci de la garantie de l'ordre public international. ${ }^{1083}$ Dans cette perspective, le Conseil de sécurité serait en effet appelé à assurer un rôle de garant de l'ordre public international, dès lors que du fait de son but et de son objet, il constitue le moyen, voire l'instrument indispensable du maintien de la paix et de la sécurité internationale. La défense de cet ordre public international doit s'effectuer, à l'instar de la sphère interne, par un organe centralisant les compétences nécessaires, en l’occurrence, le Conseil de sécurité, dans le cadre d'un cas très exceptionnel de législation unilatérale internationale à portée immédiatement obligatoire pour tous les États. C’est justement dans ce cadre que s’inscrit la création par le Conseil de sécurité du Tribunal Pénal international pour l'ex-Yougoslavie à travers la résolution 827 en mai 1993. Selon le Professeur Alain PELLET, « on peut estimer qu’en créant le tribunal pénal international pour l’ex-

1076 KOKOROKO (Komla Dodzi), « Réflexion sur le pouvoir législatif du Conseil de sécurité de l'ONU », op.cit., p.128.

1077 PELLET (Alain), "Ladaptation du droit international aux besoins changeants de la société internationale ", Académie de droit international de la Haye, Conférence inaugurale droit international public, Tiré à part du recueil des cours, tome 329,2007, p.34.

1078 Ibid,p.136.

1079 YAO-N'DRE (Paul), Droit des organisations internationales, op.cit., p.104.

1080 KOKOROKO (Komla Dodzi), « Réflexions sur le pouvoir législatif du Conseil de sécurité de l'ONU », op.cit., p.140.

1081 Ibid, p.141.

1082 Ibid, p.141.

1083 Ibid. 
Yougoslavie et le Rwanda, il avait déjà fait acte de législateur, non pas en instituant les tribunaux eux même (il s’agissait de décisions « individuelles » répondant à des situations particulières qualifiées de menace contre la paix), mais en définissant les crimes relevant de ces juridictions ». Mais dans l'affaire opposant en appel le sieur Dusco TADIC à ce Tribunal, la compétence du Conseil de sécurité à créer cette juridiction a été soulevée par l’appelant. La Chambre de première instance a ainsi récapitulé les demandes de l'appelant comme suit :

«Il est avancé que pour être légalement constitué, le Tribunal international aurait dû être créé soit par un traité, lacte consensuel des États, soit par amendement à la Charte des Nations Unies, et non par une résolution du Conseil de sécurité [...] La Charte n’a jamais envisagé que le Conseil de sécurité puisse, aux termes du Chapitre VII, établir un organe judiciaire, moins encore un tribunal pénal [...] Aucun organe politique comme le Conseil de sécurité ne peut créer un tribunal indépendant et impartial.» ${ }^{1084}$ Cette argumentation de l'appelant aussi justifiée fut-elle comporte des limites. Le Conseil de sécurité a recouru à la création d’un organe judiciaire sous la forme d'un Tribunal pénal international comme instrument pour l'exercice de sa propre fonction principale de maintien de la paix et de la sécurité, c'est-à-dire comme une mesure contribuant au rétablissement et au maintien de la paix dans l’ex-Yougoslavie. ${ }^{1085}$ Le Conseil de sécurité étend son action en adoptant des résolutions portant sur des thèmes généraux et permanents. ${ }^{1086}$ Mais, cette fonction de législateur du Conseil de sécurité est fortement remise en cause dans la doctrine.

D’emblée, il est important de souligner que la Charte des Nations Unies fait du Conseil de sécurité un organe de «réaction rapide», et non une «Assemblée délibérante.» ${ }^{1087}$ Lors du Colloque de la SFDI de 1994 sur le Chapitre VII, Alain PELLET soulignait que « la Charte impose au Conseil de sécurité de ne se prononcer que sur des situations concrètes » et « qu'il n'est nullement un législateur international. ${ }^{1088} \mathrm{Sa}$ composition restreinte constitue un obstacle majeur à sa fonction législative. En légiférant, le Conseil usurperait les fonctions de l’Assemblée générale, seul organe dont la composition internationale et des structures démocratiques pourraient donner une légitimité à des décisions d’une aussi grande portée. ${ }^{1089}$ Le Professeur KOKOROKO fait remarquer à juste titre que «le Conseil de sécurité n’est pas un parlement mondial, il n’est pas dépositaire d’une quelconque souveraineté de l'humanité, il n'est investi d’aucun pouvoir législatif.» ${ }^{1090}$ Le Professeur Alain PELLET nuance ses propos an affirmant que " les initiatives législatives qu’a prises le Conseil de sécurité ne présentent pas que des inconvénients et que, pour douteuse qu’en soit la

1084 LAGMANI (Slim), GHERAIRI (Ghazi), HAMROUNI (Salwa), Affaires et documents de droit international, Centre de Publication Universitaire, Tunis, 2005, p.248.

1085 Ibid., p.254.

1086 KOKOROKO (Komla Dodzi), « Réflexion sur le pouvoir législatif du Conseil de sécurité », op.cit., p.126.

1087 Ibid, p.133.

1088 PELLET (Alain), Colloque de Rennes sur le Chapitre VII de la Charte des Nations Unies, SFDI, Pédone, Paris, 1995, p.314.

1089 Ibid.

1090 Ibid., p.314. 
constitutionnalité au regard de la Charte, elles ont pour elles de permettre une adaptation rapide et efficace du droit international à des besoins peu contestables de la société internationale ».1091

1091 PELLET (Alain), "Ladaptation du droit international aux besoins changeants de la société internationale ", op.cit., p.36. 


\section{CONCLUSION DE LA $2^{\mathrm{e}}$ PARTIE}

Les pouvoirs qui ont été octroyés aux organes restreints sont le reflet des rapports de force au sein des organisations internationales. La configuration de ces pouvoirs traduit le rapport existant entre les organes pléniers et les organes restreints. Dans la plupart des organisations internationales, les organes pléniers composés de tous les membres de l'organisation concentrent l'essentiel des pouvoirs politiques et juridiques au détriment des organes restreints. Cependant, ce constat doit être relativisé en ce qui concerne le Conseil de sécurité de l'ONU. Au regard de la Charte de cette organisation, il se présente incontestablement comme une exception dans le droit des organisations internationales. Dans le domaine du maintien de la paix et de la sécurité internationales, qui est le but essentiel de l'ONU, il dispose des pouvoirs principaux au détriment de l'Assemblée générale qui est un organe détude. Aussi, la plupart des États appellent de tous leurs vœux la limitation des pouvoirs de cet organe à travers un contrôle juridictionnel par la juridiction principale des Nations Unies que constitue la CIJ.

Cette configuration des pouvoirs des organes restreints influence aussi leur efficacité dans la régulation des relations internationales. A la vérité, en l'absence d’un pouvoir exécutif centralisé dans la société internationale, les organes restreints devraient être en mesure de combler ce vide institutionnel. C'est justement dans cette perspective que le Conseil de paix et de sécurité de l'UA est intervenu en 2005 au Togo lors de lélection du Président Faure EYADEMA, en 2003 dans la crise du Darfour et en enfin à partir de 2002 dans la crise ivoirienne. Toutefois, cette vocation des organes restreints à se présenter comme un succédané d'un organe exécutif à l'échelle internationale est remise en cause non seulement par la politique du double standard qui règne en leur sein, mais aussi par la suprématie fonctionnelle des organes pléniers.

\section{CONCLUSION GENERALE}

Dès les premières lignes de son cours donné à l'Académie de droit international de la Haye en 1960, BOUTROS-GHALI affirmait que «l'inégalité des peuples se confond avec l'histoire même de l'Homme et, avec elle, se perd dans la nuit des temps. Elle constitue l'un des repères les plus sûrs des faits de civilisations ou de culture.» ${ }^{1092} \mathrm{Si}$ les peuples ont été toujours inégaux, il n'est guère surprenant que les organisations internationales, acteurs importants des relations entre ces peuples ne portent les stigmates de cette inégalité. Que les organes restreints des organisations internationales soient par essence des organes oligarchiques, aussi bien dans leur fonctionnement que dans la répartition de leurs pouvoirs, n’est en aucun sens en contradiction avec les réalités politiques des relations internationales. Le phénomène des inégalités de puissance entre les États

1092 BOUTROS-GHALI (Boutros), «Le principe d’égalité des États et les organisations internationales », op.cit., p.9. 
composant la société internationale trouve son terrain de prédilection dans les organes restreints des organisations internationales. ${ }^{1093}$

Malheureusement, cette inégalité entre les États a des effets négatifs sur l'efficacité des organes restreints. Leur fonctionnement est fortement marqué par la domination permanente des États ayant les contributions les plus importantes au sein des organisations internationales. Cependant, le droit international constitue avant tout un langage commun dont on espère que porté par un combat politique constant, ${ }^{1094}$ et surtout par des institutions démocratiques contribuera à instaurer une véritable égalité entre les États dans les relations internationales.

Dans cette perspective, la revendication d'une véritable démocratie au sein des organes restreints des organisations internationales pose d'emblée des problèmes dordre juridique et politique dans les relations internationales. Comme le fait observer le Professeur Monique CHEMILLIER-GENDREAU, «pour rêver d'un autre système institutionnel mondial, il est impératif de se demander d'abord dans quel monde vivons- nous et où nous voulons aller.» ${ }^{1095}$ Les organisations internationales devraient avant tout consacrer leurs efforts à diminuer lécart entre grandes et petites puissances, entre nations riches et nations pauvres. ${ }^{1096} \mathrm{Ce}$ faisant, tous les États devront avoir la même chance de participer au fonctionnement des organes restreints, ${ }^{1097} \mathrm{en}$ supprimant les pouvoirs de vote spéciaux, tous les aménagements qui offrent aux nations privilégiés de la planète des positions de choix dans ces organes. ${ }^{1098}$

Toutefois, entre la simple volonté de démocratisation des organes restreints et la réalité des relations internationales marquée par les rapports de force économique et politique entre les grandes puissances et les petits États, il y a évidemment un enseignement que l'histoire des nations retient. Pour les petits États, «le nouvel ordre international ne peut seulement s'autoriser d'un usage externe. L'incantation ne suffit point si on ne la transforme pas en un acte de développement qui s'enracine d'abord au sein des nations et des peuples pour ensuite trouver un écho et un prolongement au sein de la société internationale.» ${ }^{1099}$

La question de la démocratisation des organes restreints, qui en réalité, constitue une préoccupation légitime dans la mesure où elle remet en cause le droit de veto, la composition restreinte de certains organismes, la permanence de certains membres, le vote pondéré au sein de certaines institutions spécialisées, une certaine pratique du consensus qui est détournée de sa vocation initiale ${ }^{1100}$ doit être posée en relation avec une approche économique des relations internationales.

1093 VIRALLY (Michel), " Panorama du droit international contemporain », op.cit., p.4O.

1094 CORTEN (Olivier), « La sécurité collective, un rêve contrarié ", in Le monde diplomatique, Septembre, 2005, p.17.

1095 CHEMILLIER-GENDREAU (Monique), " Pour une organisation de la communauté mondiale », in Le monde diplomatique, Septembre, 2005, p.23.

1096 BOUTROS-GHALI (Boutros), « Le principe d'égalité et les organisations internationales », op.cit., p.69.

1097 Ibid, p.70

1098 Ibid.

1099 MAHIOU (Ahmed), "L'Algérie et les organisations internationales», AFDI, 1982, p.145.

$1100 \quad$ Ibid, p.138. 
Les États qui souhaitent la démocratisation des organes restreints devraient d'abord chercher à figurer parmi les contributeurs les plus importants au sein des organisations internationales. A ce sujet, en dépit des inégalités qui existent entre les États membres, l'UA s'est inscrite dans une orientation égalitariste entre eux. Bien que calqué sur le modèle du Conseil de sécurité de l'ONU, le Conseil de paix et de sécurité de l'UA n’a pas prévu en son sein de statut de membres permanents ni de droit de véto en faveur de certains États. Toutefois, il faut se poser la question de savoir si cette innovation favorisera l'efficacité réelle de la nouvelle organisation africaine? Il est difficile de répondre à cette question par l'affirmative dans la mesure où le fonctionnement du Conseil de paix et de sécurité pourrait être mis en mal si les États membres ne prennent pas en considération les contributions des grands États comme le Nigéria, l’Afrique du Sud, l'Egypte et l'Algérie.

Malgré l'inégalité entre les États membres, les organes restreints sont très importants dans le fonctionnement des organisations internationales. Leur efficacité apparaît surtout dans leur contribution au fonctionnement continu et régulier des organisations internationales. Contrairement aux organes pléniers qui ne peuvent se réunir que de façon périodique en raison du nombre pléthorique des membres, les organes restreints ont l'avantage d'agir plus facilement et rapidement. Le fonctionnement des organisations internationales dépend surtout de leur célérité. Mais, des améliorations peuvent être apportées à cette contribution des organes restreints dans les organisations internationales. Ainsi, au niveau du Conseil de sécurité des Nations Unies, il serait judicieux d'éviter son encombrement en revoyant le mécanisme par lequel certains États non membres sont invités à participer aux débats au sein de cet organe. En outre, la multiplication exagérée des organes restreints constitue aussi un autre facteur d'inefficacité de ceux-ci. Les États membres doivent s'efforcer de limiter le nombre d’organes restreints institués par l'acte constitutif afin de réduire les contraintes budgétaires résultant d'une telle inflation organique.

La nécessité d'un organe restreint pose inéluctablement le problème de la répartition des pouvoirs dans les organisations internationales. Les fondateurs des organisations internationales doivent trouver une juste distribution des pouvoirs politiques et juridiques entre les organes restreints et les organes pléniers. Ce problème témoigne de la volonté des États de récupérer le pouvoir de l’organisation à travers un de ces organes. La compétition entre l'Assemblée générale de l'Autorité des fonds marins et le conseil illustre parfaitement cette tension lors de la création de cette organisation. Majoritaires au sein de l’Assemblée générale, les petits États souhaitaient que les pouvoirs politiques et juridiques soient transférés à l’organe plénier. Au contraire, les grandes puissances, minoritaires dans l’organisation mais possédant les moyens technologiques nécessaires pour l'exploitation des fonds marins militaient en faveur de loctroi de ces pouvoirs au Conseil. Ainsi les rédacteurs de la Convention de Montégo Bay optèrent pour une solution médiane qui consistera à conférer les pouvoirs d’orientation des grandes décisions politiques à l'Assemblée générale. En revanche, le Conseil dominé par les grandes puissances sera lorgane disposant des 
pouvoirs spécifiques qui visent essentiellement à contrôler l'entreprise chargée d’exploiter les fonds marins.

L'efficacité des organes restreints peut être mesurée par le contenu des pouvoirs qui leur sont reconnus. Au plan politique, il n'existe pas dans l'ordre international un pouvoir exécutif suprême susceptible d'user de la force pour obliger les États à respecter les normes du droit international. Dans ces conditions, les organes restreints peuvent se présenter comme des succédanés d’un pouvoir exécutif à léchelle internationale. Généralement, les Chartes constitutives leur reconnaissent la qualité d’organe exécutif des organisations internationales. A ce titre, le Conseil de sécurité de l'ONU et le Conseil de paix et de sécurité de l'UA ont pu prendre des décisions visant la régulation des relations internationales. Il en va de même pour la Commission de l'UA qui agit de concert avec le Conseil de paix et de sécurité dans le domaine du maintien de la paix et de la sécurité internationales sur le contient; et le Conseil économique et social de l'ONU dans les domaines de la coopération économique, sociale et surtout des droits de l'homme.

$\mathrm{Au}$ plan juridique, les Chartes constitutives donnent compétence aux organes restreints dédicter des actes juridiques. Ce sont généralement des actes unilatéraux qui régissent leur fonctionnement interne et externe. Il s'agit précisément des résolutions, des recommandations et des décisions. Mais en plus des actes unilatéraux, les Chartes constitutives attribuent à certains organes restreints le pouvoir de conclure des traités. Ici également se pose le problème de l'effectivité et l’obligatoriété des actes unilatéraux vis-à-vis des États membres. Une chose pour les États membres est de reconnaître le pouvoir dédiction des actes unilatéraux aux organes restreints, mais une autre chose est d'accepter que ces actes produisent des effets dans leur ordre interne. S'il est vrai que les États font beaucoup de résistances à l'application des actes unilatéraux des organes restreints dans leur ordre interne en raison de leur souveraineté ; aujourd'hui la tendance est plutôt à l'exécution de ces actes sans par les États. Cette évolution se justifie par le principe de la primauté du droit international sur le droit interne. En vertu de cette suprématie du droit international sur le droit interne, les résolutions du Conseil de sécurité sont prises en considération par le juge interne dans certains États.

Mais les pouvoirs des organes restreints connaissent des limitations. Elles émanent des États et des organes pléniers. Les États, sujets principaux du droit international public et créateurs des organisations internationales influencent largement les pouvoirs qu'ils ont attribués aux organes restreints. L'institution d'un droit de véto à leur profit et l'extension considérable des fonctions de certains organes restreints comme le Conseil économique et social des Nations Unies conduisent à des disfonctionnements. Le droit de véto, à lui seul constitue une source de blocage dans le processus décisionnel. Un aménagement de son usage ou son attribution à d’autres États doit être envisagé au sein des organisations internationales. A cet effet, les obstacles contenus dans les Chartes constitutives doivent être levés afin de permettre une réforme fonctionnelle des organes restreints. Il s'agit des dispositions rédhibitoires telles que les articles 108 et 109 de la Charte des 
Nations Unies. Mais au-delà des intentions, la réforme des organes restreints dépend surtout de la volonté des créateurs des organisations internationales que sont les États. La réforme du Conseil de sécurité de l'ONU qui devrait ouvrir une nouvelle ère de démocratisation des relations internationales n'a pas atteint les résultats escomptés. Cette ambition se heurte toujours aux mêmes difficultés: ${ }^{1101}$

-Aucun des membres permanents n'est prêt à abandonner son siège; de plus, chacun de ces cinq membres permanents dispose, par l'article 108 de la Charte, d'un droit de véto sur toute proposition de réforme ;

- Nombre d'États (Allemagne, Japon, Inde, Brésil et bien d'autres) revendiquent pour eux un siège de membre permanent et chaque demande en suscite d'autres : la candidature de l'Inde entraîne, au nom du "pourquoi pas moi ?» celle du Pakistan ; la candidature de l'Allemagne déchaîne la fureur de l'Italie et celle du Japon, les foudres de la Chine. Les surenchères sont telles que les détenteurs présents des sièges permanents sont fondés à ne pas bouger.

De plus, les débats sur la réforme du Conseil de sécurité sont toujours demeurés très partiels ou biaisés : seule est mise sur la table la modification de la composition du Conseil de sécurité, essentiellement par l'accueil de nouveaux membres permanents. Deux questions majeures et interdépendantes restent, semble-t-il, tabou : le principe même de membres permanents, d'États bénéficiant à perpétuité d’une supériorité due à un moment historique (la victoire de 1945) et le droit de véto. Dans un système international démocratique, de tels privilèges, de telles dérogations à l'égalité sont-ils admissibles ?1102

Quant au plan de Monsieur Kofi Annan, il tente à nouveau un contournement. Il ne touche pas aux cinq membres permanents actuels et propose seulement d'ajouter de nouveaux membres permanents et non permanents. ${ }^{1103}$ Il évite soigneusement de proposer la suppression du droit de véto au Conseil de sécurité, alors que c'est par ce biais que les cinq membres permanents font la pluie et le beau temps au sein de l'ONU. A quoi servirait l'augmentation du nombre de membres au Conseil de sécurité si les cinq continuent de bloquer les sujets qui les fachent ?1104

1101 MOREAU DEFARGES (Philippe), «La réforme de l'ONU, obsédante et impossible », www.afri-ct.org/IMG/ pdf/67_Moreau_Defarges.pdf.

1102 Ibid.

1103 Ibid.

1104 La réforme des Nations Unies d’après Monsieur Kofi Annan, Secrétaire général de l'ONU, présentée dans son rapport « Dans une liberté plus grande : développement, sécurité et respect des droits de l'homme pour tous », critiques et propositions de : l'Association Amériacine des juristes (AAJ) et du Centre Europe et tiers monde, htpp:// www.aaj.org.br/, p.7. 
Malgré toutes les propositions de Monsieur Annan, il faut constater léchec de la réforme après la réunion de la soixantième session de l’Assemblée générale de l’ONU car aucune décision n’a été prise et aucune solution privilégiée. ${ }^{1105}$

Léchec jusqu' à présent répété des différentes tentatives de réforme signifie-t-il que toute réforme de l'ONU est-elle impossible ? L'histoire ne prouve rien. Ce qui a été impossible ou ce qui a semblé lêtre hier peut brutalement devenir possible demain. ${ }^{1106}$

1105 Le document final de la soixantième session de l'Assemblée générale de l'ONU du 15 septembre 2005 le prouve. Aux points 153 et 154 les États membres déclarent : «....153. Nous souhaitons- et c'est lélément central de la réforme générale de l’Organisation que nous menons- que le Conseil de sécurité soit réformé sans tarder, afin de la rendre plus largement représentatif, plus performant et plus transparent, ce qui accroîtra encore son efficacité, la légitimité de ses décisions et la qualité de leur mise en œuvre. Nous nous engageons à continuer à nous efforcer d'aboutir à une décision à cette fin, et nous prions l’Assemblée générale d'examiner, d'ici à la fin de 2005, les progrès accomplis sur cette voie.

154. Nous recommandons que le Conseil de sécurité continue à adapter ses méthodes de travail de façon à ce que les États qui n’en sont pas membres participent davantage, le cas échéant à ses travaux, à ce qu'il réponde mieux de son action devant l'ensemble des États membres et à ce qu'il fonctionne dans une plus grande transparence."

1106 MOREAU DEFARGES (Philippe), « La réforme de l'ONU, obsédente et impossible », op.cit.,www.afri-ct.org/ IMG/pdf/67Moreau Defarges.pdf. 


\section{BIBLIOGRAPHIE}

\section{I/ OUVRAGES GENERAUX}

- AMERASINGHE (C.F.), Principles of the institutional law of international organizations, Cambridge UP, 2005.

- BADIE (Bertrand), BIRNBAUM (Pierre), BRAUD (Philippe), HERMET (Guy), Dictionnaire de la science politique et des institutions politiques, Armand Colin, Paris, 1994.

- BEN ACHOUR (Rafâa), Institutions de la société internationale, Centre de Publication Universitaire, Tunis, 2004.

- $\quad$ BROWNLIE (I.), Principles of public international law, Oxford UP, 2008.

- CARREAU (Dominique),MARElla (Fabrizio),Droit international, Pédone, Paris, $11^{\mathrm{e}}$ édition, 2012.

- CAVARE (Louis), Le droit international Public positif, Tome I, Pédone Paris, 1967.

- COMBACAU (Jean) et SUR (Serges), Droit international public, Montchrestien, Paris, $9^{e}$ édition, 2010.

- CRAVEN (M.), FITZMAURICE (M.), VOGIATZI (M.), Time, history and international law, Nijhoff, Lieden, 2007.

- DECAUX (Emmanuel), Droit international public, Dalloz, $7^{\mathrm{e}}$ édition, Paris, 2010.

- DIEZ DE VELASCO VALLEJO (Manuel), Les organisations internationales, Economica, Paris, 2002.

- DJIENA WEMBOU (Michel Cyr), Le droit international dans un monde en mutation : essais écrits au fil des ans, L'Harmattan, Paris, 2003.

- DUPUY (René-Jean), La communauté internationale entre le mythe et l'histoire, Economica, UNESCO, 1986.

- DUPUY (René-Jean), Manuel sur les organisations internationales, Académie de droit international de la Haye, Martinus Nijhoff Publisher, 1998.

- GONIDEC (Pierre-François), Les organisations internationales africaines, l'Harmattan, Paris, 1987.

- GUTTIEREZ ESPADA (C.), Derecho internacional publico, Trotta, Madrid, 1995.

- GOWLLAND-DEBBAS (V), United nations sanctions and international law, Kluwer, La haye, 2001.

- KAMTO (Maurice), PONDI (Jean-Emmanuel), ZANG (Laurent), L'OUA : rétrospectives et perspectives africaines, Economica, Paris, 1990.

- $\quad$ KLABBERS (J), An introduction to international institutional law, Cambridge UP, 2002.

- MERLE (Marcel), La vie internationale, Armand Colin, $3^{e}$ édition, Paris, 1970.

- MIAJA De La MUELA (A), Introduccion al derecho internacional publico, Atlas, Madrid, 1960. 
- NGUYEN QUOC (Dinh), DAILLIER (Patrick), FORTEAU (Mathias), PELLET (Alain), Droit international public, LGDJ, Paris, $8^{\mathrm{e}}$ édition, 2009.

- PERRIN BRINCHAMBAUT (Marc), DOBELLE (Jean-François), D’HAUSSY (Marie-Reine), Leçons de droit international public, Presses de sciences po et Dalloz, Paris, 2002.

- QUAL (Lino Di), Les effets des résolutions des Nations Unies, LGDJ, Paris, 1967.

- REUTER (Paul), Institutions Internationales, Thémis, Paris, 1995.

- SAROOSHI (D.),The united nations and the development of collective security, Clarendon Press, Oxford, 1999.

- SCHACHTER (O), International law in theory and practice, Nijhoff, Dordrecht, 1991.

- SEYERSTED, Common law of international organizations, Nijhoff, Leiden, 2008.

- WODIE (Francis), Les institutions régionales en Afrique occidentale et centrale, Paris, LGDJ, 1970.

- YAO-N’DRE (Paul), Droit des organisations internationales, PUCI, Abidjan, 1996.

- YAO-N’DRE (Paul), Relations internationales, PUCI, Abidjan, 1999.

- ZARB (Antoine H.), Les institutions spécialisées du système des Nations Unies, Pédone, Paris, 1980. 


\section{II/ OUVRAGES SPECIALISES}

- BANGOURA (Dominique) et FIDIECK A BIDIAS (Emile), L'Union africaine et les acteurs sociaux dans la gestion des crises et des conflits armés, L’Harmattan, Paris, 2006.

- Chaumont (Charles), L’ONU, Que sais-je ? n 748, Paris, PUF, 1986.

- COLLIARD (Claude-Albert) et DUBOIS (Louis), Institutions internationales, Dalloz, Paris, 1995.

- COMBACAU (Jean), Le pouvoir de sanction de l'ONU : étude théorique de la coercition non militaire, Pédone, Paris, 1974.

- COT (Jean-Pierre), PELlet (Alain), FORTEAU (Mathias), La Charte des Nations Unies, Commentaire article par article, Economica, Paris, $3^{e}$ édition, 2005.

- DE SENARCLENS (Pierre), La mondialisation, théories, enjeux et débats, Dalloz, $4^{e}$ édition, 1998, 2005.

- GERBET (Pierre), Les organisations internationales, Que sais-je ?, n792, Paris, 1972.

- GONIDEC (Pierre-François), Les institutions internationales, Les cours de droit, Fascicule I, mai, 1971.

- HASBI (Aziz), ONU et ordre mondial : réformer pour ne rien changer, L'Harmattan, Paris, 2005.

- HATTO (Ronald), ONU et maintien de la paix : propositions de réforme. De l'Agenda pour la paix au rapport Brahimi, L'Harmattan, Paris, $2 \mathrm{OO6}$.

- KERBRAT (Yann), La référence au chapitre VII de la charte des Nations Unies dans les résolutions à caractère humanitaire du Conseil de sécurité, LGDJ, Paris, 1995.

- LEWIN (André) (sous la coordination), La France et l'ONU depuis 1945, Panoramiques-Corlet, Paris, 1995.

- Liniger-GOUMAZ (Max), ONU et dictatures, de la démocratie et des droits de l'homme, L'Harmattan, Paris, 1984.

- MOREAU DEFARGES (Philippes), Lordre mondial, Armand colin, Paris, 1998.

- MVELLE (Guy), l'Union Africaine, fondements, organes, programmes et actions, L'Harmattan, Paris, 2007.

- PONDI (Jean-Emmanuel) avec la collaboration de POUT (Christian Edmond Bepi), L'ONU vue d'Afrique, Maisonneuve et Larosse/ Afredit, Paris, 2005.

- DUPUY (Pierre-Marie), KERBRAT (Yann), Droit international public, Dalloz, Paris, $11^{\mathrm{e}}$ édition, 2012.

- Société Française pour le Développement du Droit International, Régionalisme et universalisme dans le droit international contemporain, Colloque de Bordeaux, Pédone, Paris, 1977.

- SUR (Serge), Relations internationales, Montchrestien, Paris, 6e édition, 2011.

- VIRAlly (Michel), L’organisation mondiale, Armand Colin, Paris, 1972. 
- ZARB (H.Antoine), Les institutions spécialisées du système des Nations Unies, Pedone, Paris, 1980 .

- ZORGBIBE (Charles), Les organisations internationales, Que sais-je ? n 8855, PUF, Paris, 1983. 


\section{III- ARTICLES DE REVUES SCIENTIFIQUES}

- AGO (Roberto), "Communauté internationale et organisation internationale », in " Manuel sur les organisations internationales ", Académie de droit internationale de La Hayes, 1988, pp.1-12.

- BEDjAOUI (Mohamed), "Du contrôle de la légalité des actes du Conseil de Sécurité ", in International law at time of perplexity, essays in honor of Shabtaï Rosenne, Boston/London, Martinus Nijhoff Publishers,1989, pp.57-110.

- BEDJAOUI (Mohamed), «Jurisprudence comparée des tribunaux administratifs internationaux en matière d'excès de pouvoir ", AFDI, 1956, pp.482-496.

- BERLIA (Georges), «Contribution à létude de la nature de la protection diplomatique », AFDI, 1957.

- BOGAERT (E. Van), "Les antinomies entre le droit international et le droit interne », RGDIP, 1968, pp.346-360.

- BOUTROS-GHALI (Boutros), "Le principe dégalité des États et les organisations internationales », RCADI, 1960- II, pp.8-7O.

- CADOUX (Charles), «L'organisation des Nations Unies et le problème de l'Afrique australe, l'évolution de la stratégie des pressions internationales ", AFDI, 1977, pp.127-174.

- CASSAN (Hervé), «Le consensus dans la pratique des Nations Unies », AFDI, 1974, pp.456485.

- CASTENADA (Jorge), « La valeur juridique des résolutions des Nations Unies », RCADI, Tome 129, 1970-I.

- CHAUMONT (Charles), «L'équilibre des organes politiques des Nations Unies et la crise de l'organisation », AFDI, 1965, pp.428-446.

- CHRISTAKIS (Théodore) et BANNELIER (Karine), « Acteur vigilant ou spectateur impuissant? Le contrôle exercé par le Conseil de sécurité sur les États autorisés à recourir à la force. ", Ares, $\mathrm{n}^{\circ}$ 55, Fascicule 3, Volume XXI, mai 2005, pp.32-43.

- COICAUD (Jean-Marc), "Réflexions sur les organisations internationales et la légitimité internationale : contraintes, pathologies et perspectives ", Revue internationale des sciences sociales, $\mathrm{n}^{\circ} 170,2001 / 4, \mathrm{pp} .573-587$.

- CONFORTI (Benedetto), «Rôle de l'accord dans le système des Nations Unies », RCADI, Tome 142, 1974-II, pp. 2O4-288.

- CORTEN (Olivier), " La participation du Conseil de sécurité à lélaboration, à la cristallisation ou à la consolidation des règles coutumières », Ares, n55, Fascicule 3, Volume XXI, mai 2005, pp.70-87.

- DE ORUE Y ARREGUI (José Ramon), « Le régionalisme dans l'organisation internationale », RCADI, 1935-III, pp.1-93. 
- DRAGO (Roland), " La pondération dans les organisations internationales ", AFDI, 1956, pp.529-547.

- DUPUY (René-Jean), "L'organisation internationale et l'expression de la volonté générale ", RGDIP, 1957, p.527-578.

- DUPUY (René-Jean), «Le droit des relations entre les organisations internationales », RCADI, 1960- II, p.457-589.

- FISHER (Georges), « Lorganisation des pays exportateurs de pétrole », AFDI, 1961.

- FLAUSS (Jean- François), «Les réserves aux résolutions des Nations Unies », RGDIP, 1981-1.

- FOCSANEANU (Lazare), «Le droit interne de l’organisation des Nations Unies », AFDI, 1957, pp.315-348.

- FROMONT (Michel), "Labstention dans les votes au sein des organisations internationales », AFDI, 1961, pp.492-523.

- GAJA (Georgio), « réflexion sur le rôle du Conseil de Sécurité dans le nouvel ordre mondial », RGDIP, 1993, pp.297-320.

- GESLIN (Alban), «Le pouvoir d'habilitation du Conseil de sécurité : La délégation des pouvoirs du Conseil de sécurité aux organisations internationales ", Ares, n55, fascicule 3, Volume XXI, mai 2005, pp.14-31.

- GOLDSMITH (Jack L.) and POSNER (Eric A.), « Moral and legal rhetoric in international relations: a rational choice perspective ", in John M. Olin law and Economics working paper, n 108 ", http:// www.law.uchicago.edu/ Publications/ working/ index. Html.

- GOLDSMITH (Jack Landman) and LEWINSON (Daryl J.), « Law for States: International law, Constitutional law, Public law », Haward law school public law and legal theory working paper series, $\mathrm{n}^{\circ}$ 9-16, http:// ssrn.com/ abstract $=1340615$.

- GUILHAUDIS (Jean-François), "Considérations sur la pratique de l'union pour la paix », AFDI, 1981.

- GUZMAN (Andrew T.), "International law: a compliance based theory », in UC Berkeley public and legal theory working paper, $\mathrm{n}^{\circ} 47$, april 2001.

- GUZMAN (Andrew T.), «The case for international antitrust », UC Berkeley School of law, public law and legal theory research paper, $\mathrm{n}^{\circ}$ 128, 2003.

- GUZMAN (Andrew T.), "The design of international agreements ", in University of Pennsylvannia law review, November 2004.

- GUZMAN (Andrew T.), "International tribunal: a rational choice », in University of Pennsylvannia law review, Volume 157, 2008.

- GUZMAN (AndrewT.) and (MEYER Thymothy L.), « International soft law », Electronic copy available at:http://ssrn.com/ abstract=1868280, 2009.

- GUZMAN (Andrew T.), «The consent problem in international law», Electronic copy available at: http:// ss.com/ abstract=1862354, 2011. 
- HERNDL (K.), « Reflections on the role of the security council of the United nations », RCADI, 1990-IV, Volume 223, p.289-396.

- KOKOROKO (Komla Dodzi), «Réflexions sur le pouvoir législatif du Conseil de sécurité de l'ONU », RBSJA, décembre 2007, n¹9, pp. 123-153.

- KRANZ (Jerzy), «Le vote pondéré dans les organisations internationales », RGDIP, 1981, pp. 313-346.

- LAGMANI (Slim), « Le nouvel ordre politique international et son impact sur le droit international ", in Affaires et documents de droit international, Centre de Publication Universitaire, Tunis, 2005, pp.7-2O.

- LANFRANCHI (Marie-Pierre), «La valeur juridique en France des résolutions des Nations Unies », AFDI, 1997, pp.31-57.

- LAGRANGE (Evelyne), "Le Conseil de sécurité peut-il violer le droit international ? ", Ares, $\mathrm{n}^{\circ} 55$, Fascicule 3, Volume XXI, mai 2005, pp.88-101.

- LEWIN (André), « La triade contraignante, une nouvelle proposition de pondération des voix aux Nations Unies », RGDIP, 1984, pp.349-359.

- MAHAIM (Ernest), « Lorganisation permanente du travail », RCADI, 1964-III, tome 4, pp.65223.

- MAHIOU (Ahmed), «L'Algérie et les organisations internationales », AFDI, 1982, pp.125-145.

- MANKIEWICZ (R.H), « Augmentation des membres de l'OACI », AFDI, 1961, pp.445-450.

- MAUPAIN (Francis), « La protection internationale des travailleurs et la libéralisation du commerce mondial : un lien ou un frein ? ", RGDIP, 1996-I, pp.45-100.

- MAZERON (Florent), «Le contrôle de la légalité des décisions du Conseil de sécurité, un bilan après les ordonnances Lockerbie et l'arrêt Tadic », in Revue québécoise de droit international, Volume 10 (1997), pp. 105-136.

- MERLE (Marcel), «Le pouvoir règlementaire des institutions internationales », AFDI, 1958, pp. 341-360.

- MILES (Thomas J) and POSNER (Eric A.), «Which states enter into treaties, and why?», in John M.Olin Law and Economics working paper, $\mathrm{n}^{\circ} 420$, Public law and legal theory paper, $\mathrm{n}^{\circ} 225$, August 2008, http:// ssrn.com/abstract=12111777.

- MONACO (Ricardo), "Les principes régissant la structure et le fonctionnement des organisations internationales », RCADI, Tome 156, 1977, pp. 80-191.

- NDIAYE (Biram), «L'incapacité temporaire de l'Assemblée générale à élire le dixième membre non permanent du Conseil de sécurité ", AFDI, 1980, pp.483-5O2.

- ORSONI (Gilbert), «Le retrait des États-Unis et la crise de l'OIT », RGDIP, 1979, pp.689-746.

- OUGUERGOUZ (Fatsah), «La tragédie rwandaise du printemps 1994 : quelques considérations sur les premières réactions de l’organisation des Nations Unies », RGDIP, 1996-I, pp. 149-177. 
- PELLET (Alain), « Ladaptation du droit international aux besoins changeants de la société internationale ", Académie de droit international de la Haye, Conférence inaugurale, session de droit international public, 2007, Martinus Nijhoff publishers, Leiden/ Boston, 2008, p.13-47.

- PELlET (Alain), " Le droit international dans l'ombre de l'empire », Baltic Yearbook of international law, Volume 6, 2006, p.27-37.

- PEREZ GONZALEZ (Manuel), " Les organisations internationales et le droit de la responsabilité », RGDIP, 1988, pp. 63-102.

- POSNER (Eric A.) and SYKES (Alan O.), «An economic analysis of state and individual responsibility under international law», in John M. Olin law and economics working paper, $\mathrm{n}^{\circ} 279$, and in Stanford university law school, paper $\mathrm{n}^{\circ} 318$.

- QUENEDEUC (Jean- Pierre), «A propos de la composition du Conseil de Sécurité », RGDIP, 1995-4, pp.945-960.

- SMOUTS (Marie-Claude), "Réflexions sur les méthodes de travail du Conseil de sécurité ", AFDI, 1982, pp. 601-612.

- SOREL (Jean-Marc), "L'ONU et l'Irak : le vil plomb ne s'est pas transformé en or pur », RGDIP, 2004, pp. 845-854.

- SOREL (Jean-Marc), « Le caractère discrétionnaire des actes du Conseil de sécurité : remarques sur quelques incertitudes partielles », Ares, n55, Fascicule 3, volume XXI, mai 2005, pp.11-13.

- SOUBEYROL (Jacques), "Considérations sur la crise financière de l'ONU », RGDIP, 1967, pp. 675-716.

- STARITA (MASSIMO), « L'occupation de l'Irak, le Conseil de Sécurité, le droit de la guerre et le droit des peuples à disposer d'eux-mêmes », RGDIP, 2004-4, pp. 881-916.

- STERN (Brigitte), «Vers la mondialisation juridique ? Les lois Helms- Burton et d'AmatoKennedy», RGDIP, 1996-II, pp.979-1003.

- SYKES (Alan O.), "The economics of public international law ", in John M. Olin law and Economics working Paper, $\mathrm{n}^{\circ}$ 216, 2n $2^{\text {nd }}$ series, Chicago, july, 2004.

- TAVERNIER (Paul), «Le processus de reforme des Nations Unies. Du rapport Bertrand (1985) au rapport des $18 », R G D I P, 1968$, pp. 305-334.

- TERCINET (Josiane), «Le pouvoir normatif du Conseil de sécurité : Le Conseil de sécurité peut-il légiférer? », Ares, n55, Fascicule 3, Volume XXI, ami 2005, pp. 70-87.

- TOUBLANC (Alix), "Larticle 103 et la valeur juridique de la charte des Nations Unies ", RGDIP, 2004-2, pp. 439-462.

- VALTICOS (Nicolas), «Les Conventions de l'organisation internationale du travail à la croisée des anniversaires », RGDIP-I, pp.5-43.

- VIRALlY (Michel), " La valeur juridique des recommandations des organisations internationales », AFDI 1956, pp. 66-96. 
- VIRALlY (Michel), «Le rôle politique du Secrétaire général des Nations Unies », AFDI, 1958, pp. 360

- VIRALlY (Michel), « Le testament politique de Dag Hammarskjoeld », AFDI, 1961, pp. 354380.

- VIRALLY (Michel), « Panorama du droit international public », RCADI, V, Tome 183, 1983.

- VISSCHER (Charles de), « Observations sur l'effectivité en droit international public », RGDIP, 1958, p.601-609. 


\section{IV- TEXTES JURIDIQUES}

- Acte Constitutif de l'Union Africaine, adopté le 11 juillet 2000 à Lomé (Togo).

- Avis consultatif de la CIJ du 11 avril 1949, relatif à la réparation des dommages subis au service des Nations Unies.

- Charte des Nations Unies, San Francisco, le 26 juin 1945.

- Constitution de l'OIT, Genève, mai, 2004.

- Convention de Vienne sur le droit des traités, du 23 mai 1969.

- Décision n019/CC/SG du Conseil Constitutionnel de la République de Côte d’Ivoire, du 06 décembre 2006, portant sur le contrôle de constitutionnalité de la résolution 1721 du Conseil de sécurité.

- Déclaration de Hararé sur la réforme du Conseil de Sécurité, juin 1997.

- Recueil des arrêts, avis consultatifs et ordonnances de la CIJ, 1971.

- Règlement du Conseil d'administration de l'OIT, Génève, mai 2004.

- Règlement intérieur de la Commission de l'Union africaine.

- Statut de la CIJ

- Statut de la Commission de l’UA.

\section{V/ ARTICLES PARUS SUR INTERNET}

- ADJOVI (Roland), « Togo, un changement anticonstitutionnel savant et un nouveau test pour l'Union Africaine », Actualité et droit international, www.ridi.org/adi, février 2003.

- Association américaine des juristes, «La réforme des Nations Unies, d'après Monsieur Kofi ANNAN, Secrétaire général de l'ONU, présenté dans son rapport «Dans une liberté plus grande : développement, sécurité et respect des droits de l'homme pour tous ", htpp:// www.aaj. org.br.

- BEN ACHOUR (Rafâa), «L'ONU et l'Irak II », Actualité et droit international, www.ridi.org/adi, novembre 2004.

- BEN ACHOUR (Rafâa), « La résolution 1546 du Conseil de Sécurité ou l’apogée de l’art de la fiction », Actualité et droit international, www.ridi.org/adi, juillet 2004 (version septembre 2004).

- CHARVIN (Robert), « La guerre anglo-américaine contre l’Irak et le droit international, Apocalypse law", Actualité et droit international, www.ridi.org/adi, avril 2003.

- FAYE (André), « Réflexion sur la réforme du Conseil de sécurité », www.univ-Reims.fr/labos/ CERI/article-andre-faye- $h$ tm, avril 2004.

- FERRAJOlO (Ornella), « La pratique et la règle de droit, réflexion à propos de la seconde guerre du golf», Actualité et droit international, www.ridi.org/adi, mai 2004. 
- FREIESLEBEN (Jonas Von), «Reform of the Security Council », in Center for United Nations reform education, New York, p.3, 2008, http://www.centerforunreform.org/node/308.

- KUGEL (Alischa), "Reform of the Security Council- A new approch? », FES Briefing paper, http:// library.fes.de/pdf-files/iez/global/06696, 2009.

- LAGHMANI (Slim), « Du droit international au droit impérial ? réflexion sur la guerre en Irak», www.ridi.org/adi, avril 2003.

- LAGHMANI (Slim), «Faut-il rire du droit international ou le pleurer ? ", Actualité et droit international, www.ridi.org/adi, février 2003.

- LUND (Jacob S.), " Moving towards text-Based Negociations ? ", Center for United Nations reform education, New York, http://www. Centerforunreform.org/node/ 415, 2010.

- LUND (Jacob S) et SAFRAN-HON (Daniel), " Third Round of intergouvernemental Negotiations on United Nations Security council reform conclude ", in Center for United Nations reform education, New York, http://www.Centerforunreform.org/node/407, 2009.

- MOREAU DEFARGES (Philippe), "La réforme de l'ONU, obsédante et impossible », www. afri-ct.org/IMG/pdf/67 Moreau Defarges.pdf.

- NAHORY (céline) et PAUL (James), «To contribute to the maintenance of international peace and security ", in The quest for regional representation. Reforming the United Nations Security council, critical currents ${ }^{\circ} 4$, Uppsala, Dag Hammarskjold Foundation, p.30, http://www.dhf. uu.se/critical currents $\mathrm{n}^{\circ} 4 \mathrm{html}, 2008$.

- PELLET (Sarah), « De la raison du plus fort ou comment les États -Unis ont (ré) inventé le droit international et leur droit constitutionnel », Actualité et droit international, www.ridi.org/adi, juin 2002.

- TAVERNIER (Paul), «Soixante après : la réforme du Conseil de Sécurité des Nations Unis estelle possible? ", Actualité et droit international, www.ridi.org/adi, août 2003.

- TRACHSELER (Daniel), "Réforme du Conseil de sécurité : un nœud gordien ? ", in Center for Security Studies, ETH Zürich, n 72, p.2, http://www.ssn.ethz.ch/Aktuell/CSS-Ananysen/ Detail/?Ing=fr-id=114413.

\section{VI- TRAVAUX DE THESE ET MEMOIRE}

- AUDOIN (Jean-Pierre), L’Autorité internationale des fonds marins (D’après les Travaux de la troisième Conférence des Nations Unies sur le droit de la mer), Thèse pour le Doctorat d'État en droit, Nice, septembre 1979.

- COUlibaly (Drissa), Le droit de véto à l'ONU, Mémoire pour le Diplôme d'Etudes Approfondies de droit public, l’Université Félix Houphouet Boigny d’Abidjan-Cocody, 19961997. 


\section{VII-ARTICLES DE PRESSE}

- ALBALA (Nuri) : "Maudit droit de véto ", in Le monde diplomatique ", n618, à la page 23.

- CHEMillier-Gendreau (Monique), " Pour une organisation de la communauté mondiale ", in Le monde diplomatique, $\mathrm{n}^{\circ} 618$, à la page 22 et 23.

- CORTEN (Olivier), «La sécurité collective, un rêve contrarié »,in Le monde diplomatique, ${ }^{\circ}$ 618, septembre 2005, à la page 17.

- Interview de l'ex-Président Libyen Mouhamar KADHAFI, paru dans le Quotidien ivoirien «Fraternité Matin », dans sa livraison n 12252, du Samedi 10 au dimanche 11 septembre 2005, aux pages 2 et 3 .

- Interview de Monsieur John Bolton, ancien Ambassadeur des États-Unis à l'ONU, paru dans le Quotidien ivoirien «Le Courrier d'Abidjan », dans sa livraison n¹071, du Mardi 24 juillet 2007, à la page 7 .

- LECOUTRE (Delphine), "L'Afrique et la réforme des Nations Unies ", in Le monde diplomatique, juillet 2005, p.10-14.

- «Présidence de la Commission de l'UA, la Côte d'Ivoire plurielle et en voie de réconciliation après sept mois de guerre, entend aller en rangs serrées derrière son candidat Amara ESSY ", paru dans le Quotidien ivoirien « Fraternité Matin, dans sa livraison du mercredi 21 mars 2003, à la page 24 .

- POWER (Samantha), "Réformer les Nations Unies ", in Le monde diplomatique ", n 618, septembre 2005, à la page 1 et la suite à la page 18 et 19 .

- RUIZ-DIAZ (Hugo), «Une tribune pour les pays du Sud», in Le monde diplomatique, nº18, à la page 20 et 21 . 


\section{INDEX DES TERMES UTILISES}

A

Acte constitutif: $10,14,21,27,33,58,60,61,62$, $64,66,77,78,81,82,91,140,185,186$, 192, 200, 207, 208, 216, 220, 231, 239, $249,261,262,290,297$

Actes juridiques: 203, 218, 219, 224, 236, 238, $241,242,243,244,248,287$

C

Compétences administratives: 296

Compétences spécialisées: 300

Conclusion des traités: 235

Contrôle de constitutionnalité: 278, 279, 281, $282,286,287,338$

Convention de Vienne sur le droit des traités: $21,231,338$

Crise de légitimité: 256, 257, 262, 263, 266

Critère de la contribution aux activités de lorganisation: 106, 107, 108, 114, 115, $116,117,118,119$

D

Décisions: $11,26,33,51,53,62,71,82,83,96$, $98,125,126,127,131,133,134,136$, $144,150,169,172,180,181,185,186$, 193, 195, 224, 229, 230, 231, 240, 244, $245,249,251,268,269,285,296,297$. $301,302,311,312,313,319,335$

Déconcentration des organisations internationales: 60,61

Délégation de compétence: 50, 75, 76, 269, 270

Démocratie représentative: $71,72,73,74,75$, $79,81,82,83,85,86$
Droit de véto: $16,27,125,126,127,128,129$, $154,165,166,167,168,169,170,171$, $172,173,246,276,277,278,340$

\section{E}

Effectivité: 243, 244, 247, 248, 337

Efficacité dans les organisations internationales: 10

Efficacité des organes restreints: 150

Elargissement des organes restreints: 151, 163

\section{G}

Gouvernement à l'échelle internationale: 11, 199, 202

$\mathbf{H}$

Hégémonie des États membres: 102

Impératifs des groupes régionaux: 266, 268

Inflation des organes: 62

\section{M}

Mesures générales: 220, 222, 223

Mesures individuelles: 220, 222, 223

\section{O}

Oligarchies d'États: 104, 105, 114

Ordre politique: $139,158,161,167,281,334$

Organes administratifs: 26

Organes dérivés: 26

Organes originaires: 26

Organes pléniers: $7,8,9,12,27,28,48,49,52$, $59,61,73,75,76,86,87,88,90,92,94$, 96, 101, 102, 142, 173, 177, 178, 179, 180, 181, 184, 185, 187, 188, 192, 195, 196, 197, 220, 225, 238, 254, 280, 287, 288, 290, 291, 292, 295, 296, 298, 299, 302, 303, 304, 306, 307, 308 
Organes politiques: $26,50,163,188,189,192$, $193,195,229,241,281,312,315,332$

Organisation de réunions périodiques: 41, 44, 47

Organisations à vocation générale: 24,58

Organisations d'intégration: 24

Organisations de coopération: 24

Organisations régionales: 23, 86, 188

Organisations spécialisées: 24,58

Organisations universelles: 23, 33

\section{$\mathbf{P}$}

Pouvoir d'évocation: 306, 307, 308

Pouvoir de substitution: 304, 306, 307

Pouvoir législatif: 206, 315, 319, 334

Pouvoirs opérationnels: 204, 214

Principe d'égalité: 9, 12, 13, 14, 15, 16, 57, 73, $122,124,132,162,187,321,332$

Principe de la continuité du service public: 41

Principe de la répartition géographique équitable: $113,119,120,124$

Principe de la spécialité: 22

\section{$\mathbf{R}$}

Recommandations: $67,91,185,186,187,189$, $194,201,212,213,221,224,227,228$, $235,239,240,300,302,313,337$

Reformes fonctionnelles: 164, 165, 170

Reformes structurelles: 139, 140, 164, 165

Règle de l'unanimité: 132, 133, 134, 266

Règle de la majorité: 133, 134, 135

Règle du consensus: 133, 135, 136

Répartition des pouvoirs politiques: 177, 178, $179,185,195,197$

Représentation permanente: $41,44,45,46,47$, 80
Résolutions: 44,73,135, 136, 187, 190, 204,205, 206, 224, 225, 226, 227, 228, 238, 240, $241,244,246,247,248,250,251,257$, $262,263,269,284,285,312,313,316$, $319,329,331,332,333,335$

S

Souveraineté: $19,22,25,58,77,85,199,202$, $228,245,246,248,249,255,319$

Statut de membres permanents: $16,46,124$, $125,129,147,165,166,167,168,170$, 171,172

\section{$\mathbf{T}$}

Traité constitutif: 20,342

Traité multilatéral: 21, 153

V

Vote pondéré: $12,128,129,130,131,132,322$, 334 


\section{Table des matières}

I. Abréviations des organisations internationales. $\ldots \ldots \ldots \ldots \ldots \ldots \ldots \ldots \ldots \ldots \ldots \ldots$

II. Abréviations utilisées dans la bibliographie $\ldots \ldots \ldots \ldots \ldots \ldots \ldots \ldots \ldots \ldots \ldots \ldots \ldots$

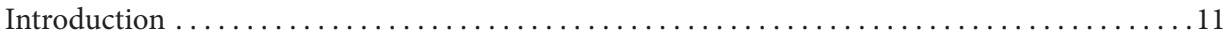

Première partie: Les fondements de l'institution des organes restreints dans les organisations

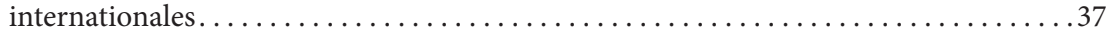

Titre I : La recherche de l'efficacité des organisations internationales. . . . . . . . . . . . 39

Chapitre I : Les apports fonctionnels des organes restreints dans les organisations

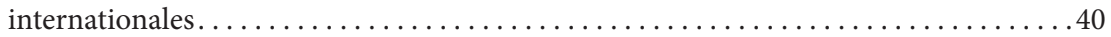

Section I : La célérité dans l'exécution des missions des organisations internationales . . . . . . 41

Paragraphe I : La mise en œuvre de la continuité du service public dans les organisations

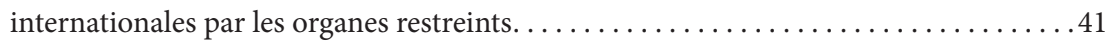

A : Lorganisation de réunions périodiques dans les organes restreints $\ldots \ldots \ldots \ldots \ldots 2$

B : La réprésentation permanente des États membres dans les organes restreints . . . . . . . 44

Paragraphe II : La disponibilité des organes restreints face aux situations d'urgence dans les

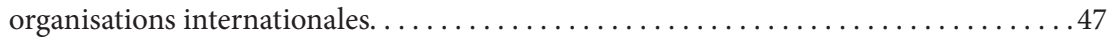

A : La mise en place d'un système de réaction rapide fondé sur les organes restreints $\ldots \ldots \ldots 47$

B : Les limites du système de réaction rapide fondé sur les organes restreints $\ldots \ldots \ldots \ldots \ldots$

Section II : La rationalisation fonctionnelle des organisations internationales par les organes

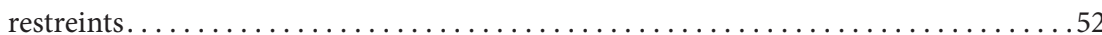

Paragraphe I : La déconcentration des activités des organisations par le truchement des organes

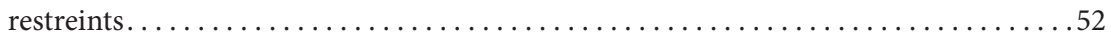

A : La pluralité des missions des organisations internationales. . . . . . . . . . . . . 53

B : La nécessité de la déconcentration des organisations internationales. . . . . . . . . . 55

Paragraphe II : Les limites à la déconcentration des organisations internationales par les organes

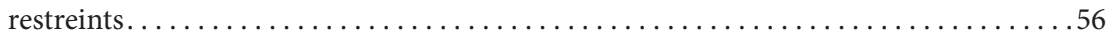

A : L'inflation des organes restreints dans les organisations internationales . . . . . . . . 56

B : La présence d’organes restreints d'apparat dans les organisations internationales $\ldots \ldots \ldots 60$

Chapitre II : Les apports au plan politique des organes restreints au fonctionnement des

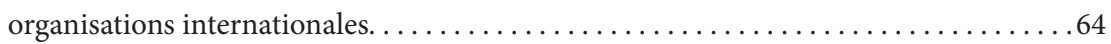

Section I : La mise en place d'une démocratie representative dans les organes restreints . . . . . 65

Paragraphe I : Le contenu de la notion de démocratie représentative dans les organes restreints .65

A : La clarification de la notion de démocratie représentative . . . . . . . . . . . . . 65

B : Les conditions de mise en œuvre de la démocratie représentative $\ldots \ldots \ldots \ldots \ldots \ldots \ldots$

1/ La délégation de compétence des membres des organes pléniers aux organes restreints. . . . . . 68 
2/ La légitimation des membres des organes restreints par les États membres

Paragraphe II : La portée de la démocratie représentative dans les organisations internationales.72

A : Le rôle de la démocratie représentative dans le fonctionnement des organisations

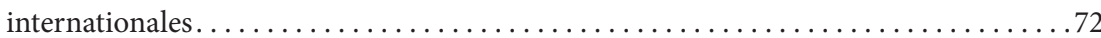

B : Les lacunes de la démocratie représentative dans les organisations internationales........73

Section II : L'indispensable complémentarité politique entre les organes pléniers et les organes

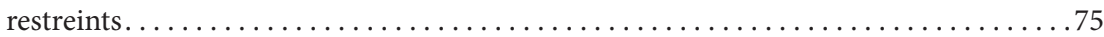

Paragraphe I : Les manifestations de la complémentarité ....................... 76

A : Le statut d’organes délibérants des organes pléniers............................ 76

B : Le statut d'organes exécutifs des organes restreints . . . . . . . . . . . . . . . . . . 77

Paragraphe II : Les limites de la complémentarité entre organes pléniers et organes restreints . . 79

A : Le dysfonctionnement des organes restreints. . . . . . . . . . . . . . . . . . . . . . 79

B : L'instrumentalisation des organes restreints par les États membres $\ldots \ldots \ldots \ldots \ldots \ldots \ldots 2$

Titre II : La problématique de l'hégémonie des États membres dans les organes restreints . . . . 87

Chapitre I : Lémergence des oligarchies d'États dans les organes restreints. . . . . . . . . . 88

Section I : La notion d'oligarchie d'États dans les organes restreints. . . . . . . . . . . . . 89

Paragraphe I : Les critères de formation des oligarchies d'États dans les organes restreints . . . .89

A : La contribution aux activités de l'organisation.............................. 89

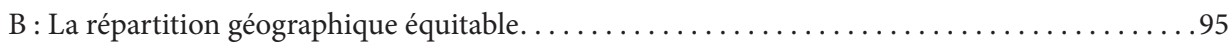

Paragraphe II : La portée de ces deux critères dans les organes restreints . . . . . . . . . . 97

A : La finalité aristocratique du critère de la contribution aux activités de l'organisation ....... 98

$B$ : Le caractère sélectif du critère de la réprésentation géographique équitable $\ldots \ldots \ldots \ldots \ldots 0$

Section II : La consécration des inégalités entre les États dans les organes restreints........... 102

Paragraphe I : La catégorisatrion des membres des organes restreints.................. 102

A : Le statut de membres permanents des grandes puissances . . . . . . . . . . . . . . 103

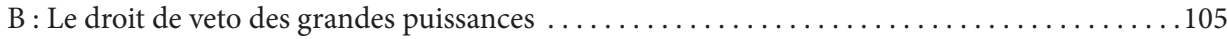

Paragraphe II : L’instauration de conditions de votes inéquitables . . . . . . . . . . . . 108

A : La pratique de la pondération des voix .................................... 109

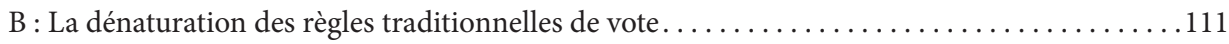

(1)Lallègement de la règle de l'unanimité par celle de la majorité .......................112

(2) Le caractère contraignant de la règle du consensus pour les petits États au sein des organes

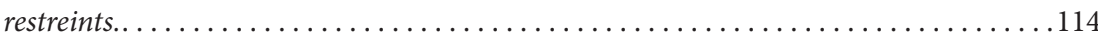

Chapitre II : La contestation de l'hégémonie des grandes puissances dans les organes restreints 116 SECTION I : La nécessité d'une réforme structurelle des organes restreints. . . . . . . . . 117

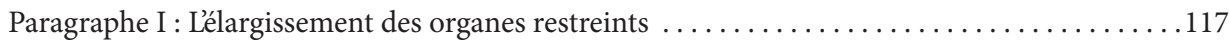

A : Un élargissement justifié par la réprésentativité des petits États ...................117 
B : Un élargissement imposé par les mutations politico-économiques dans les relations

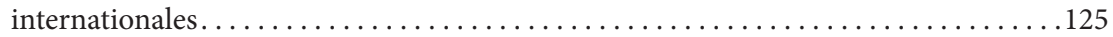

Paragraphe II : Les obstacles à lélargissement des organes restreints ................. 128

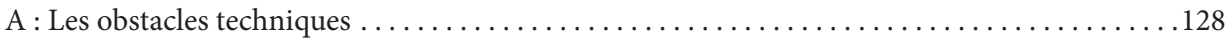

1) La remise en cause de l'efficacité des organes restreints........................... 128

2) La modification de la Charte constitutive des organisations internationales..............130

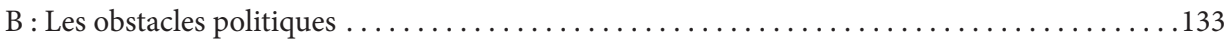

1) Le bouleversement des équilibres politiques au sein des organes restreints. . . . . . . . . . 133

2) L’incompatibilité du modèle démocratique étatique au sein des organisations internationales ..137

Section II : Lexigence de reformes fonctionnelles des organes restreints. . . . . . . . . . 138

Paragraphe I : La révendication de la suppression du droit de veto et du statut de membres permanents par les petits États . . . . . . . . . . . . . . . . . . . . . . . . . . . . 139

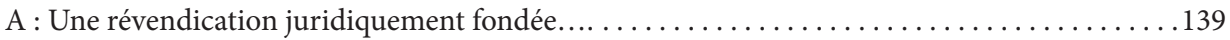

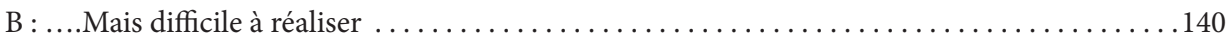

Paragraphe II : La logique de rupture du Conseil de paix et de sécurité de l'UA . ........... 142

A : L'inexistence du droit de veto et du statut de membres permanents.................. 142

B : Les conséquences sur le fonctionnement du Conseil de paix et de sécurité . . . . . . . . 143

Conclusion de la première partie ...................................... 145

Deuxième partie: Lordonnancement des pouvoirs des organes restreints. . . . . . . . . . 147

Titre I : La détermination des pouvoirs des organes restreints..................... 149

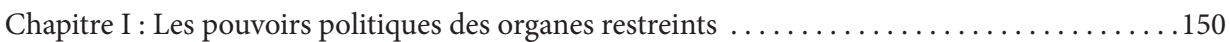

Section I : La répartition des pouvoirs politiques entre les organes restreints et les organes

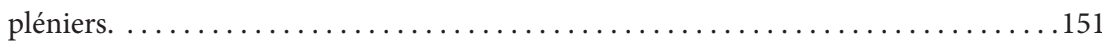

Paragraphe I : La compétition pour le contrôle du pouvoir politique entre les organes pléniers et

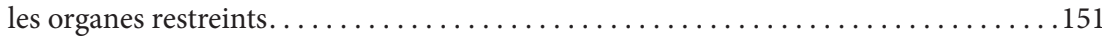

A : La révendication du pouvoir politique par les organes pléniers $\ldots \ldots \ldots \ldots \ldots \ldots \ldots \ldots 151$

B : La volonté de récupération des pouvoirs politiques par les organes restreints . . . . . . . 153

Paragraphe II : La configuration des pouvoirs politiques entre les organes restreints et les organes

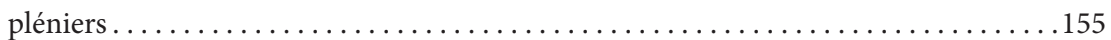

A : Les rapports de hiérarchie politique entre les organes restreints et les organes pléniers .....155

1- La suprématie politique des organes pléniers sur les organes restreints . . . . . . . . . . . 155

2- La suprématie politique des organes restreints sur les organes pléniers................. 158

B : Les rapports d'équilibre politique entre les organes restreints et les organes pléniers. . . . . 161

1- Les manifestations de léquilibre politique ..................................... 161

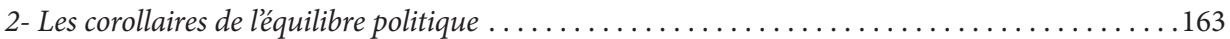

Section II : Létendu des pouvoirs politiques des organes restreints . . . . . . . . . . . . 164 
Paragraphe I : Les organes restreints, un ersatz d'un gouvernement à l'échelle internationale . . 164 A : La vocation des organes restreints à réprésenter un pouvoir exécutif à léchelle

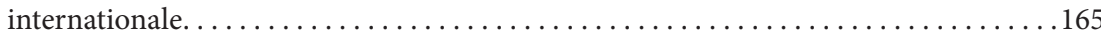

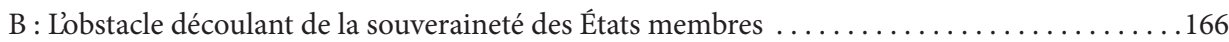

Paragraphe II : La régulation des relations internationales par les organes restreints ........ 168

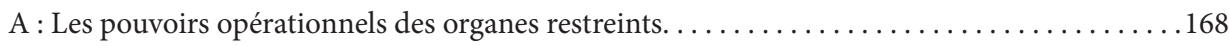

B : Les carences des pouvoirs opérationnels des organes restreints $\ldots \ldots \ldots \ldots \ldots \ldots \ldots \ldots \ldots \ldots$

Chapitre II : Les pouvoirs juridiques des organes restreints..................... 177

Section I : La nomenclature des actes juridiques des organes restreints $\ldots \ldots \ldots \ldots \ldots \ldots \ldots$

Paragraphe I : Les actes juridiques liés au fonctionnement interne de l'organisation. . . . . . 178

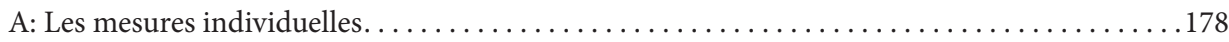

B: Les mesures generales . . . . . . . . . . . . . . . . . . . . . . . . . . . . . . . . . 179

Paragraphe II : Les actes juridiques liés aux activités extérieures des organisations

internationales............................................

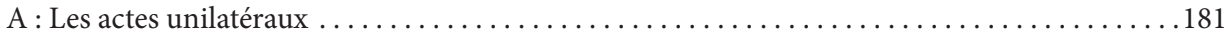

$1 /$ Les résolutions des organes restreints . ...................................... 181

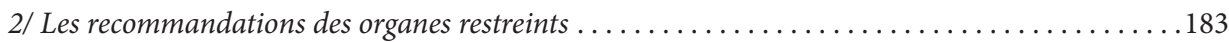

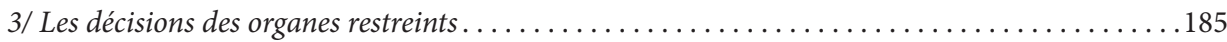

B : La conclusion des traités . . . . . . . . . . . . . . . . . . . . . . . . . . . . 186

Section II : La valeur juridique des actes des organes restreints ..................... 190

Paragraphe I : La valeur des actes des organes restreints dans le fonctionnement interne des organisations internationales. . . . . . . . . . . . . . . . . . . . . . . . 190

A : La valeur juridique des actes édictés entre organes hiérarchisés .................. 191

B : La valeur juridique des actes édictés entre organes indépendants................... 192

Paragraphe II : La portée des actes des organes restreints dans les relations avec les États . . . . 194

A : L’effectivité des actes juridiques des organes restreints dans l’ordre interne des États. . . . . 194

B : L’autorité des actes juridiques des organes restreints dans l'ordre interne des États . . . . . . 199

Titre II : Les limites aux pouvoirs des organes restreints ........................ 205

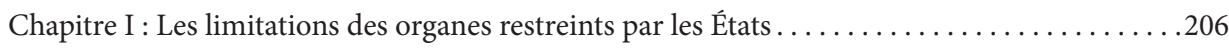

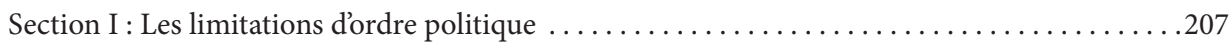

Paragraphe I : La crise de légitimité des pouvoirs des organes restreints...............207

A : Les manifestations de la crise de légitimité des pouvoirs des organes restreints . . . . . . 207

B : Les implications de la crise de légitimité des pouvoirs des organes restreints . . . . . . . 213

Paragraphe II : Les difficultés politiques liées au fonctionnement des organes restreints. . . . 2215

A : Les impératifs des groupes régionaux étatiques. . . . . . . . . . . . . . . . . 216

B : La pratique de la délégation de compétence des organes restreints par les États. . . . . . . 218

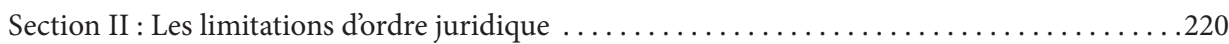


Paragraphe I : Les limitations imposées par les États à travers les chartes constitutives .......220

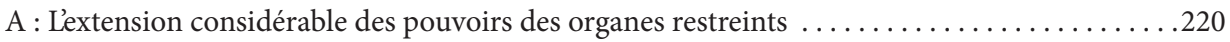

B : Les restrictions aux pouvoirs des organes restreints découlant du droit de véto..........222

Paragraphe II : Les limitations souhaitables pour les États: Le contrôle de constitutionnalité des

actes du Conseil de sécurité des Nations unies........................224

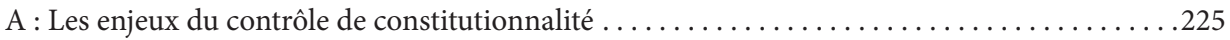

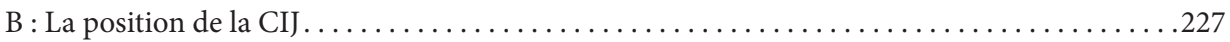

Chapitre II : Les limitations des pouvoirs des organes restreints par les organes pléniers......231

Section I : La dépendance des organes restreints vis-à-vis des organes pléniers . . . . . . . 232

Paragraphe I : La désignation des membres des organes restreints par les organes pléniers.... 232

A : Lélection des membres des organes restreints par les organes pléniers $\ldots \ldots \ldots \ldots \ldots \ldots 232$

B : La politisation de lélection des membres des organes restreints.. . . . . . . . . . . 233

Paragraphe II : La réduction des compétences des organes restreints par les organes pléniers . .236

A : Des compétences purement administratives .......................... 236

B: Des compétences essentiellement spécialisées........................... 238

Section II : Le contrôle des organes restreints par les organes pléniers $\ldots \ldots \ldots \ldots \ldots \ldots \ldots . \ldots \ldots 1$

Paragraphe I : Le pouvoir hiérarchique des organes pléniers sur les organes restreints. . . . . . 241

A : Le pouvoir de substitution des organes pléniers aux organes restreints............ 242

B : Le pouvoir d'évocation des organes pléniers sur les organes restreints............... 243

Paragraphe II : L'exception du Conseil de sécurité des Nations unies...................244

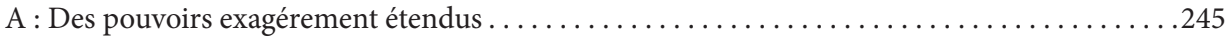

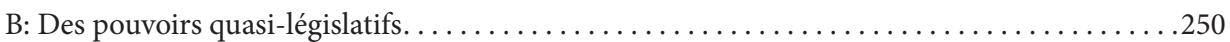

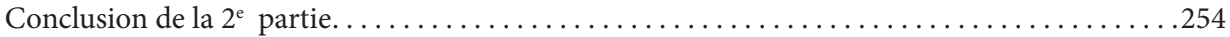

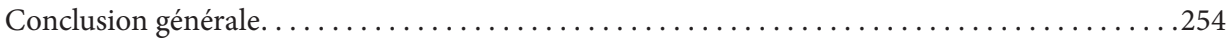

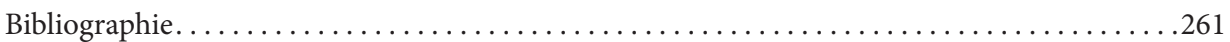

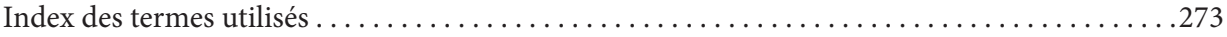

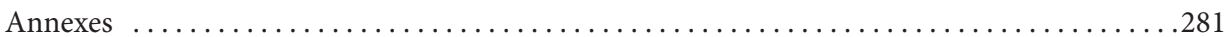


ANNEXES 


\begin{tabular}{lcc}
\hline PAYS & MILLIONS & EN \% \\
\hline États-Unis & 363 & 24,48 \\
\hline Japon & 280 & 18,88 \\
\hline Allemagne & 124 & 8,36 \\
\hline Royaume-Uni & 88 & 5,93 \\
\hline France & 87 & 5,87 \\
\hline Italie & 70 & 4,72 \\
\hline Espagne & 36 & 2,43 \\
\hline Chine & 29 & 1,96 \\
\hline Mexique & 27 & 1,82 \\
\hline Corée du Sud & 26 & 1,75 \\
\hline Pays-Bas & 24 & 1,62 \\
\hline Australie & 23 & 1,55 \\
\hline Brésil & 22 & 1,48 \\
\hline Suisse & 17 & 1,15 \\
\hline Sous Total 14 pays & 1216 & 82 \\
\hline
\end{tabular}

ANNEXE I :

TABLEAU DES 14 PREMIERS CONTRIBUTEURS AU BUDGET ORDINAIRE DE L'ONU 


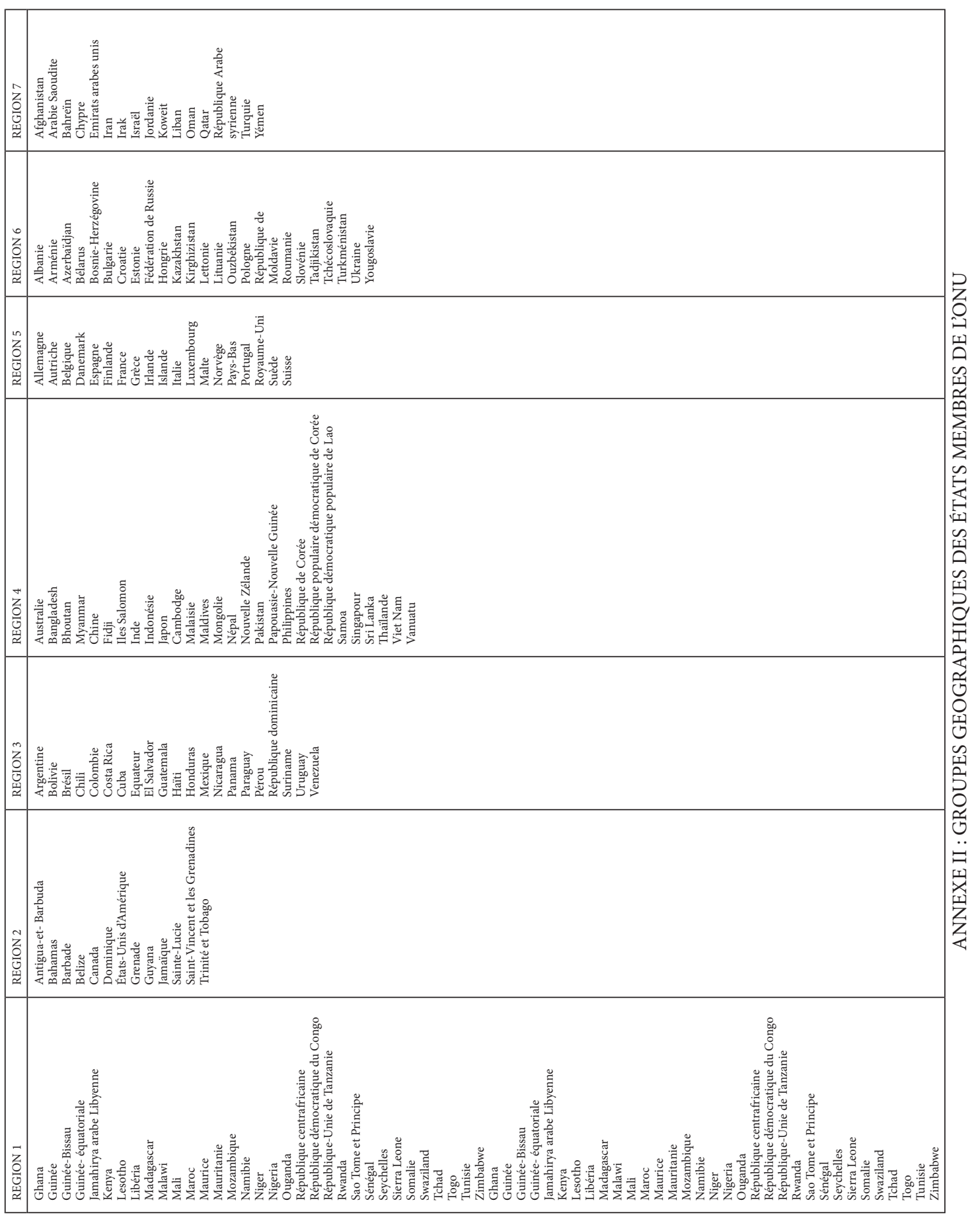

